\title{
THE IWASAWA MAIN CONJECTURE FOR HILBERT MODULAR FORMS
}

\author{
XIN WAN \\ Mathematics Department, Columbia University, New York, 10027, USA; \\ email: xw2295@math.columbia.edu
}

Received 22 February 2014; accepted 17 January 2015

\begin{abstract}
Following the ideas and methods of a recent work of Skinner and Urban, we prove the one divisibility of the Iwasawa main conjecture for nearly ordinary Hilbert modular forms under certain local hypotheses. As a consequence, we prove that for a Hilbert modular form of parallel weight, trivial character, and good ordinary reduction at all primes dividing $p$, if the central critical $L$-value is zero then the $p$-adic Selmer group of it has rank at least one. We also prove that one of the local assumptions in the main result of Skinner and Urban can be removed by a base-change trick.
\end{abstract}

2010 Mathematics Subject Classification: 16W10 (primary); 16D50 (secondary)

\section{Introduction}

1.1. The conjecture. Let $p$ be an odd prime and $F$ a totally real number field where $p$ is unramified. Suppose that $[F: \mathbb{Q}]=d$. Let $\mathcal{K}$ be a totally imaginary quadratic field extension of $F$ such that each prime $v$ of $F$ above $p$ is split. Suppose that

$(\triangle) \mathcal{K}$ is not contained in the narrow Hilbert class field of $F$ and all primes $v$ ramified in $F / \mathbb{Q}$ are split in $\mathcal{K}$.

We let $F_{\infty}$ be the cyclotomic $\mathbb{Z}_{p}$ extension of $F$. The Galois group, which we denote as $\Gamma_{F}$, is isomorphic to $\mathbb{Z}_{p}$. Let $\mathcal{K}_{\infty}^{-}$be the maximal abelian anticyclotomic (the complex conjugation acting by -1 on the Galois group) $\mathbb{Z}_{p}$-extension of $\mathcal{K}$ unramified outside $p$ with Galois group denoted as $\Gamma_{\mathcal{K}}^{-}$. This is isomorphic to $\mathbb{Z}_{p}^{d}$. Write $\mathcal{K}_{\infty}^{+}$for $F_{\infty} \mathcal{K}$ with $\Gamma_{\mathcal{K}}^{+}$the Galois group over $\mathcal{K}$ (identified with $\Gamma_{F}$ ).

(C) The Author 2015. This is an Open Access article, distributed under the terms of the Creative Commons Attribution licence (http://creativecommons.org/licenses/by/4.0/), which permits unrestricted re-use, distribution, and reproduction in any medium, provided the original work is properly cited. 
Let $\mathcal{K}_{\infty}:=\mathcal{K}_{\infty}^{-} \mathcal{K}_{\infty}^{+}$and $\Gamma_{\mathcal{K}}:=\operatorname{Gal}\left(\mathcal{K}_{\infty} / \mathcal{K}\right)$. This is a Galois extension with Galois group $\mathbb{Z}_{p}^{d+1}$. Conjecturally (Leopoldt) this is the maximal unramified outside $p$ abelian $\mathbb{Z}_{p}$-extension of $\mathcal{K}$. We define $\Lambda_{\mathcal{K}}:=\mathbb{Z}_{p}\left[\left[\Gamma_{\mathcal{K}}\right]\right]$. For any $A$ a finite extension of $\mathbb{Z}_{p}$, define $\Lambda_{\mathcal{K}, A}:=A\left[\left[\Gamma_{\mathcal{K}}\right]\right]$. We define more Iwasawa algebras $\Lambda_{F}$, $\Lambda_{\mathcal{K}}^{-}, \Lambda_{\mathcal{K}}^{+}, \Lambda_{\mathcal{K}, A}^{-}, \Lambda_{\mathcal{K}, A}^{+}$, in an analogous way. We let $\boldsymbol{\varepsilon}_{F}$ be the canonical character $G_{F} \rightarrow \Gamma_{F} \hookrightarrow \Lambda_{F}^{\times}$of $G_{F}$ and $\varepsilon_{\mathcal{K}}$ be the composition $G_{\mathcal{K}} \rightarrow \Gamma_{\mathcal{K}} \hookrightarrow \Lambda_{\mathcal{K}}^{\times}$. Let $\Psi_{F}$ or $\Psi_{\mathcal{K}}$ be $\varepsilon_{F}$ or $\varepsilon_{\mathcal{K}}$ composed with the reciprocity map of class field theory (normalized by the geometric Frobenius).

We fix topological generators for each group above:

$$
\gamma:=\operatorname{rec}_{F}\left(\prod_{v \mid p}(1+p)_{v}\right), \quad \gamma^{+}:=\operatorname{rec}_{\mathcal{K}}\left(\prod_{v \mid p}(1+p, 1+p)_{v}^{1 / 2}\right)
$$

for $\Gamma_{F}$ ad $\Gamma_{\mathcal{K}}^{+}$and

$$
\left\{\gamma_{v, i}^{-}\right\}_{v, i}
$$

for $\Gamma_{v}^{-}$with $\left\{\gamma_{v, i}^{-}\right\}_{i}$ being a set of $\left[F_{v}: \mathbb{Q}_{p}\right]$ elements with $\gamma_{v, i}^{-} \in \operatorname{Im}\left(\operatorname{rec}_{\mathcal{K}}\left(\mathcal{K}_{v}\right)\right)$. Here rec means the reciprocity map of class field theory normalized by the geometric Frobenius. Let $\Gamma_{v}^{-}$be the $\mathbb{Z}_{p}$-span of $\left\{\gamma_{v, i}^{-}\right\}$.

Let $\Sigma$ be a finite number of primes of $F$. We can use unitary groups to study Iwasawa theory for Hilbert modular forms. Let $f(\mathbf{f})$ be a nearly ordinary Hilbert modular form (or Hida family with the normal domain $\mathbb{I}$ as coefficient ring). Let $L / \mathbb{Q}_{p}$ be a finite extension containing all Fourier coefficients of $f$ and $\chi$ a $\mathcal{O}_{L^{-}}$ valued character of $\mathcal{K} \times \backslash \mathbb{A}_{\mathcal{K}}^{\times}$. Then, by results of Wiles [51], the restriction to $G_{v}$ of the Galois representation associated to $f$ for each $v \mid p$ is isomorphic to an upper triangular representation $V$ where the one-dimensional subspace $V_{v}^{+}$ has some prescribed Galois action. One can define its Selmer group $\operatorname{Sel}_{f, \mathcal{K}, \chi}^{\Sigma}$ and its Pontryagin dual $X_{f, \mathcal{K}, \chi}^{\Sigma}$ (see Section 2 for details). For $\Sigma$ containing all the bad primes, we construct in Section 7.3 a $p$-adic $L$-function $\mathcal{L}_{f, \mathcal{K}, \chi}^{\Sigma} \in \mathcal{O}_{L}\left[\left[\Gamma_{\mathcal{K}}\right]\right]$ $\left(\mathcal{L}_{\mathbf{f}, \mathcal{K}, \chi}^{\Sigma} \in \mathbb{I}\left[\left[\Gamma_{\mathcal{K}}\right]\right]\right)$. We can formulate the following main conjecture as follows.

Conjecture 1. As ideals of $\mathcal{O}_{L}\left[\left[\Gamma_{\mathcal{K}}\right]\right]\left(\mathbb{I}\left[\left[\Gamma_{\mathcal{K}}\right]\right]\right)$,

$$
\left(\mathcal{L}_{f, \mathcal{K}, \chi}^{\Sigma}\right)=\operatorname{char}_{f, \mathcal{K}, \chi}^{\Sigma}, \quad\left(\mathcal{L}_{\mathbf{f}, \mathcal{K}, \chi}^{\Sigma}\right)=\operatorname{char}_{\mathbf{f}, \mathcal{K}, \chi}^{\Sigma} .
$$

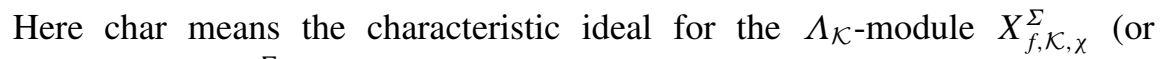
$\mathbb{I}\left[\left[\Gamma_{\mathcal{K}}\right]\right]$-module $\left.X_{\mathbf{f}, \mathcal{K}, \chi}^{\Sigma}\right)$. We can construct a nonintegral $p$-adic $L$-function $\tilde{\mathcal{L}}_{\mathbf{f}, \mathcal{K}, \chi}^{\Sigma}$ in great generality. (This is an element in $F_{\mathbb{I}}\left[\left[\Gamma_{\mathcal{K}}\right]\right]$ instead of $\mathbb{I}\left[\Gamma_{\mathcal{K}}\right]$ which is also interpolating special values of the $L$-functions with a slightly different interpolation formula; see Section 7.) This is enough for proving the characteristic-zero results (Theorem 7). However, we need certain Gorenstein 
properties of some Hecke algebras to construct the integral $p$-adic $L$-function $\mathcal{L}_{f, \mathcal{K}, \chi}^{\Sigma}$ that appears in the conjecture above. Let us briefly discuss this issue. Let f be an I-valued Hida family of nearly ordinary Hilbert modular eigenforms with tame level $M$ for II some finite extension of some weight space (to be defined in the text). Let $\mathfrak{m}_{\mathrm{f}}$ be the maximal ideal of the full Hecke algebra $\mathbb{T}(M, \mathbb{I})$ with $\mathbb{I}$ coefficients corresponding to $\mathbf{f}$. Let $\mathbb{T}_{\mathfrak{m}_{f}}:=\mathbb{T}(M, \mathbb{I})_{\mathfrak{m}_{\mathfrak{f}}}$ be the localization. Then we say that it is Gorenstein if $\operatorname{Hom}_{\mathbb{I}}\left(\mathbb{T}_{\mathfrak{m}_{\mathfrak{f}}}, \mathbb{I}\right)$ is free of rank one over $\mathbb{T}_{\mathfrak{m}_{\mathfrak{f}}}$ as a $\mathbb{T}_{\mathfrak{m}_{\mathfrak{f}}}$-module.

\subsection{Main results.}

DEFINITION 2. We give the following definitions.

- (irred) The residual Galois representation $\bar{\rho}_{f}$ of $f$ is irreducible.

- (dist) For $V=\rho_{f}$ and each prime $v \mid p$, the $\mathcal{O}_{L}^{\times}$-valued characters giving the actions of $G_{F, v}$ on $V_{v}^{+}$and $V / V_{v}^{+}$are distinct modulo the maximal ideal of $\mathcal{O}_{L}$.

We always assume that (irred) and (dist) hold for our residual representation $\bar{\rho}_{f}$. We write $\left(\operatorname{irred}_{\mathcal{K}}\right)$ to mean that the restriction to $G_{\mathcal{K}}$ of the residual Galois representation is absolutely irreducible.

These conditions are used in various places in the proof. First of all (irred) is used in constructing Galois representations for Hida families of Hilbert modular forms. Second, they are part of the conditions to ensure that a certain local Hecke algebra is Gorenstein. Third, ( $\left.\operatorname{irred}_{\mathcal{K}}\right)$ and (dist) are used in the 'lattice construction' to construct elements in the dual Selmer groups. Finally, in the proof of Theorem 100, we used a modularity-lifting result to prove that a certain automorphic form is a CAP form (meaning that its associated Galois representation is the same as that of a Klingen Eisenstein series) under these assumptions.

Our first theorem (Theorem 101) is one divisibility of the multivariable main conjecture similar to the one proved in [44].

THEOREM 3. Suppose that $p \geqslant 5$. Let L be a finite extension of $\mathbb{Q}_{p}$ and $\mathbb{I}$ a local normal domain and a finite integral extension of the 'parallel weight space' (to be defined in Section 2.3.2) $\Lambda_{W, \mathcal{O}_{L}}^{\mathrm{para}}$. Let $\mathbf{f}$ be an $\mathbb{I}$-adic ordinary Hilbert modular eigenform over $F$ of tame level $M$ (a finite idele) with trivial character, such that some specialization of it is an ordinary cusp form of even parallel weight $\kappa_{0} \geqslant 2$, and trivial nebentypus. Suppose that $(\triangle)$ is true and that $M=M^{+} M^{-}$with $M^{+}$ divisible only by primes split in $\mathcal{K}$ and $M^{-}$divisible only by primes of $F$ inert in $\mathcal{K}$. Suppose also that the following hold. 
- $\left(\operatorname{irred}_{\mathcal{K}}\right)$ and (dist) hold for the residual Galois representation $\bar{\rho}_{\mathbf{f}}$ associated to $\mathbf{f}$.

- The assumptions of Theorem 8 hold.

- $M^{-}$is square free and its number of prime factors is congruent to d modulo 2.

- The residual representation $\bar{\rho}_{\mathbf{f}}$ is ramified at all $v \mid M^{-}$.

Let $\Sigma$ be any finite set of primes containing $p$. Then we have the containment of fractional ideals.

$$
\operatorname{char}_{\mathcal{K}_{\infty}}^{\Sigma}(\mathbf{f}) \subseteq\left(\mathcal{L}_{\mathbf{f}, \mathcal{K}}^{\Sigma}\right)
$$

In the text we construct the $\Sigma$-imprimitive $p$-adic $L$-function for $\Sigma$ containing all bad primes. This is integral (that is, in $\mathbb{I}\left[\left[\Gamma_{\mathcal{K}}\right]\right]$ ). By putting back the Euler factors at $\Sigma$ we may construct the three-variable $p$-adic $L$-functions for general $\Sigma$. But we do not know if they are integral. So we use a fractional ideal in the statement of the above theorem. Here we only consider 'parallel' Hida family $\mathbf{f}$ which is the Zariski closure of the set of points with parallel weights and trivial characters. This is due to our knowledge about the anticyclotomic $\mu$-invariant. In fact there is a small mistake in [44, 12.3.2], where they claimed that the $\mu$-invariant for the $\Sigma$-imprimitive $p$-adic $L$-function is 0 from the same property for the original $L$-function. However at nonsplit primes the local Euler factors do contribute nontrivially to the $\mu$-invariant (split primes are OK). So it is necessary to compare to Hida's construction in [15] for the argument, and in our paper we are only able to make such comparison along the 'parallel weight' Hida family. It is still possible, however, to prove the result for a general Hida family by constructing the full (not $\Sigma$-imprimitive) $p$-adic $L$-function (for example in the forthcoming work [7]). Therefore we still do the computation for a general Hida family.

In fact, using the results for the other side divisibility of the anticyclotomic main conjecture using Heegner points, we can prove the equality in the main conjecture as in [44] under more technical conditions. See [32] for example. We leave this for interested readers. However, we are unable to prove the main conjecture over $F$ due to the lack of analogs of results of Kato.

Our next result (Theorem 103) states that the main theorem of [44, Theorem 3.29] is true without the third assumption in [44].

THEOREM 4. Suppose that $p \geqslant 5$. Let $f \in S_{\kappa}(M, L), 2 \mid \kappa \geqslant 2$ be a p-ordinary cuspidal eigenform with trivial character, $p \nmid M$, and $L \subset \overline{\mathbb{Q}}_{p}$ a finite extension of $\mathbb{Q}_{p}$. Suppose that 
- (irred) and (dist) hold for $\rho_{f}$.

Then, for any set of primes $\Sigma$,

$$
\operatorname{char}_{\mathbb{Q}_{\infty}, L}^{\Sigma}(f)=\left(\mathcal{L}_{f}^{\Sigma}\right)
$$

in $\Lambda_{\mathbb{Q}, \mathcal{O}_{L}} \otimes_{\mathbb{Z}_{p}} \mathbb{Q}_{p}$. If, furthermore,

- there is an $\mathcal{O}_{L}$-basis of $T_{f}$ with respect to which the image of $\rho_{f}$ contains $\mathrm{SL}_{2}\left(\mathbb{Z}_{p}\right)$, and

- there exists a real quadratic extension $F / \mathbb{Q}$ such that

- $p$ is unramified in $F$,

- any prime $\ell$ dividing $M$ such that $\ell \equiv-1(\bmod p)$ is inert in $F$, and any other prime divisors of $M$ is split in $F$, and

- the canonical period of $f$ over $F$ is a p-adic unit times the square of its canonical period over $\mathbb{Q}$,

then the equality holds in $\Lambda_{\mathbb{Q}, \mathcal{O}_{L}}$.

REMARK 5. We will use the trick of passing to the base change to a real quadratic extension to prove the theorem. Unfortunately, for this trick to work we will face the issue of comparing the periods, at least when we are concerned with the main conjecture without inverting $p$. This problem is in general difficult.

Our last result is a characteristic-zero one which does not need the Gorenstein properties mentioned above (Theorem 102). We first state the following conjecture.

CONJECTURE 6. Let $F$ be a totally real field, $p$ be an odd prime unramified in $F, \kappa \geqslant 2$ an even integer, and $g$ a Hilbert modular cuspidal eigenform over $F$ of parallel weight $\kappa$ and trivial character. Suppose that $g$ has good ordinary reduction at all primes above $p$. Assume moreover that if $[F: Q]$ is even then there is at least one prime $v$ of $F$ such that $\pi_{g, v}$ is not a principal series. If $L(g, \kappa / 2) \neq 0$ then $H_{f}^{1}\left(F, \rho_{g}^{*}((2-\kappa) / 2)\right)=0$.

This conjecture is proved when $\kappa=2$ in [38, Theorem B]. Note that the assumptions in [38] are satisfied by the description of $G_{p}$ action on $\rho_{g}$. For general $\kappa$ this conjecture is not known but we expect the method of [3] to work in the totally real case as well.

THEOREM 7. Let $F$ be a totally real number field. Let $p$ be an odd rational prime unramified in $F$. Let $f$ be a Hilbert modular form over $F$ of even parallel 
weight $\kappa_{0}$ and trivial character. Let $\rho_{f}$ be the p-adic Galois representation associated to $f$ such that $L\left(\rho_{f}, s\right)=L(f, s)$. Suppose that

(i) $f$ is good ordinary at all primes dividing $p$,

(ii) assumption (irred) holds for $f$, and

(iii) if $[F: \mathbb{Q}]$ is even and the global sign of $f$ is -1 , then the automorphic representation of $f$ is not a principal series in at least one finite place.

Suppose moreover that Conjecture 6 is true for $F, p, \kappa=\kappa_{0}$ in our theorem, and any $g$ satisfying the assumption of Conjecture 6. If the central critical value $L\left(f, \kappa_{0} / 2\right)=0$, then the Selmer group $H_{f}^{1}\left(F, \rho_{f}^{*}\left(\left(2-\kappa_{0}\right) / 2\right)\right)$ is infinite. Here $\rho_{f}^{*}$ means the Pontryagin dual $\operatorname{Hom}_{\mathbb{Z}_{p}}\left(\rho_{f}, \mathbb{Q}_{p} / \mathbb{Z}_{p}\right)$.

Note that (dist) is ensured by other assumptions because the two characters giving the diagonal actions of the inertial group at $p$ are the trivial character and the cyclotomic character. In the special case that $F=\mathbb{Q}$, Theorem 7 is essentially proved in [44], though our result is slightly more general (in particular, we do not need to assume that $f$ is special or even square integrable at any finite place).

In the case when the root number is -1 , Theorem 7 is a result of Zhang and Nekovar (in fact conditions (iii) is made to apply their results). We prove it when the root number is +1 . In fact, our theorem, combined with the parity result of Nekovar, implies that, when the order of vanishing is even and at least two, then the rank of the Selmer group is also at least two. Also note that the method of [43] does not seem to generalize to the totally real field case.

In order to prove Theorem 7 , we need to choose a CM extension (that is a totally imaginary quadratic extension) $\mathcal{K}$ of $F$ and make use of the unitary group $\mathrm{U}(1,1)_{/ F}$ which is closely related to $\mathrm{GL}_{2}$. We embed $f$ into a Hida family $\mathbf{f}$ and use some $\mathrm{CM}$ character $\psi$ to construct a family of forms on $\mathrm{U}(1,1)$. Then our proof consists of four steps. (1) From this family on $U(1,1)$ we construct a $p$-adic family of Klingen Eisenstein series on $\mathrm{U}(2,2)$, such that the constant term is divisible by the $p$-adic $L$-function of $\mathbf{f}$ over $\mathcal{K}$. (2) We prove that (the Fourier expansion of) the Klingen Eisenstein family is coprime to the $p$-adic $L$ function by a computation using doubling methods. (3) We use the results about the constant terms in step 1 to construct a cuspidal family which is congruent to the Klingen Eisenstein family modulo the $p$-adic $L$-function. (4) We pass to the Galois side, using the congruence between the Galois representations for the Klingen Eisenstein family and the cuspidal family to prove the theorem.

We record here a theorem of Fujiwara which gives sufficient conditions for $\mathbb{T}_{\mathfrak{m}_{\mathfrak{f}}}$ to be Gorenstein.

THEOREM 8 (Fujiwara). Let $\bar{\rho}$ be the residual Galois representation associated to $\mathbf{f}$. Suppose that the following hold. 
- $p \geqslant 3$ and $\left.\bar{\rho}\right|_{F\left(\zeta_{p}\right)}$ is absolutely irreducible. When $p=5$ the following case is excluded: the projective image $\bar{G}$ of $\bar{\rho}$ is isomorphic to $\mathrm{PGL}_{2}\left(\mathbb{F}_{p}\right)$ and the $\bmod p$ cyclotomic character $\bar{\chi}_{\text {cycle }}$ factors through $G_{F} \rightarrow \bar{G}^{a b} \simeq \mathbb{Z} / 2$.

- (dist) is true.

- There is a minimal modular lifting of $\bar{\rho}$.

- In the case when $d:=[F: \mathbb{Q}]$ is odd, Ihara's lemma is true for Shimura curves.

- For each finite place $v, q_{v} \not \equiv-1(\bmod p)$ if $\left.\bar{\rho}_{\mathbf{f}}\right|_{I_{F v}}$ is absolutely irreducible.

Then the ring $\mathbb{T}_{\mathfrak{m}_{f}}$ is Gorenstein.

This is [9, Theorems 11.1 and 11.2].

Many of our arguments are straightforward generalizations of [44]. However we do all the computations in the adelic language instead of the mixture of classical and adelic language of [44]. This simplifies the computations somewhat since we no longer need to compare the classical and adelic pictures. The required nonvanishing modulo- $p$ results of some special $L$-values are known thanks to the recent work of Hsieh [24] and Hung [26]. Also we use Hida's work on the anticyclotomic main conjecture to compare the CM periods and canonical periods for some auxiliary CM forms. To construct the cuspidal family in step (3) we generalize the geometric argument in [44] 6.3. (In the case when $F \neq \mathbb{Q}$ we need to restrict to a certain subfamily of the whole weight space to have freeness of the nearly ordinary forms over the (sub) weight space and surjectivity of the restricting to the boundary map.) To adapt the argument to the totally real case our choices for Fourier coefficients and Eisenstein series also differ from [44] slightly.

The paper is organized as follows (for convenience we keep the argument parallel to that in [44]). In Section 2 we recall the notion of Hilbert modular forms and record some results on Iwasawa theory for their Selmer groups. In Section 3 we recall some results about $p$-adic automorphic forms and Hida theory for the group $U(2,2)$. Sections $4-8$ (corresponding to steps (1) and (2)) are parallel to [44, Ch. 9-13] and we do the local and global calculations to deduce the required $p$-adic properties needed in Section 9. We prove our main theorems in Section 9 (corresponding to step (4)).

\section{Backgrounds}

2.1. More notation. We fix a CM type $\Sigma_{\infty}$ for $\mathcal{K}$ which means that the disjoint union $\Sigma_{\infty} \cup \Sigma_{\infty}^{c}$ is the set of all Archimedean places of $\mathcal{K}$. We sometimes write $\Sigma_{\infty}$ for the set of Archimedean places of $F$ as well. Fix $\iota_{p}: \mathbb{C} \simeq \mathbb{C}_{p}$. For each $v \mid p$ 
there is a unique place $w$ above $v$ whose corresponding Archimedean place is in $\Sigma_{\infty}$. The set of such places is a $p$-adic CM type of $\mathcal{K}$. We define $\mu_{p^{\infty}}$ as the set of roots of unity with order powers of $p$. Let $\delta_{\mathcal{K}}, \mathfrak{d}=\mathfrak{d}_{F}$ be the differents of $\mathcal{K}$ and $F$. Let $D=D_{\mathcal{K}}=\operatorname{Nm}_{\mathcal{K} / F}\left(\delta_{\mathcal{K}}\right)$ (not the discriminant of $\mathcal{K}$ !). We write $D_{v}$ for the $v$ component of it. Let $\tilde{\delta}_{\mathcal{K}}$ be the different from $\mathcal{K}$ to $F$ and $\tilde{D}_{\mathcal{K}}=\mathrm{Nm}_{\mathcal{K} / F}\left(\tilde{\delta}_{\mathcal{K}}\right)$. We denote $N$ to be the level of $f$ and $M$ the prime to $p$ part of it. Here $N, M$, $\delta_{\mathcal{K}}, \mathfrak{d}, D_{\mathcal{K}}, D_{F}$ are all elements in the ideles of $F, \mathcal{K}$, or $\mathbb{Q}$ supported at the finite primes (also the $M_{\mathcal{D}}$ defined later). This is much more convenient when working in the adelic language. For each prime $v$ of $F$ we write $\varpi_{v}$ for a uniformizer and $q_{v}$ for the cardinality of the residual field. For each $v \mid p$ we suppose that $p^{r^{v}} \| N_{v}$ (we save the notation $r_{v}$ for other use). Let $e_{v}$ be the inertial degree of $F_{v} / \mathbb{Q}_{p}$. We usually adopt the convention that $\xi_{p}\left(p^{r_{p}}\right)=\prod_{v \mid p} \xi_{v}\left(p^{r_{v}}\right)$. Also we write $\xi^{c}(x):=\xi(\bar{x})$ and $\bar{\xi}(x)=\overline{\xi(x)}$. We use the following convention: if $v$ is nonsplit in $\mathcal{K} / F$ then for an integer $r$ we write $\lambda^{r}=\left(\varpi_{w}^{r}\right)$ where $\varpi_{w}$ is the uniformizer of a prime $w$ of $\mathcal{K}$ above $v$. If $v$ is split then for a pair of integers $r=\left(r_{1}, r_{2}\right)$ we write $\lambda^{r}:=\left(\varpi_{v}^{r_{1}}, \varpi_{v}^{r_{2}}\right)$. For a character say $\xi$ we often write $\mathfrak{f}_{\xi}$ for the conductor of it. We assume that $\mathcal{K} / F$ is split at all primes dividing $\mathfrak{d}_{F}$. This assumption makes the computation of Fourier-Jacobi coefficients easier. Let $h=h_{F}$ be the narrow ideal class number of $F$. We divide the ideal classes $C l(\mathcal{K})$ into $I_{1} \sqcup \cdots \sqcup I_{h}$ corresponding to the image of the norm map to $C l_{n}(F)$ and suppose that $I_{1}$ are those mapping to the trivial class. (Here $n$ stands for narrow.) We assume that $\mathcal{K}$ is disjoint from the narrow Hilbert class field of $F$ and thus it is easy to see that the norm map above is surjective. Also we write $\langle f, g\rangle$ to be the integration of $f \cdot \bar{g}$ along $\mathrm{U}(1,1)(F) \backslash \mathrm{U}(1,1)\left(\mathbb{A}_{F}\right)$. We denote $e_{F}: \mathbb{A}_{F} / F \rightarrow \mathbb{C}^{\times}$ the standard additive character such that $e_{F}\left(x_{\infty}\right)=\exp \left(2 \pi i \sigma_{\sigma} x_{\sigma}\right)$ for $\sigma$ running over all Archimedean places of $F$. For any $\mathcal{O}_{F}$-algebra $R$ we define $S_{n}(R):=$ $\left\{A \in M_{n}\left(R \otimes_{\mathcal{O}_{F}} \mathcal{O}_{\mathcal{K}}\right), \bar{S}={ }^{t} S\right\}$.

For $f$ and $g$ Hilbert modular forms such that the product of the central characters of $f$ and $\bar{g}$ is trivial then we denote $\langle,\rangle_{\mathrm{GL}_{2}}$ to be the inner product on $\mathrm{GL}_{2}$ (integration over $\mathrm{GL}_{2}(F) \mathbb{A}_{F}^{\times} \backslash \mathrm{GL}_{2}\left(\mathbb{A}_{F}\right)$; note that we need to mod out the center here). We also write, for example, $\langle,\rangle_{\mathrm{U}_{\mathcal{D}}},\langle,\rangle_{\mathrm{GL}_{2}, \Gamma_{0}(N)}$ the inner product with respect to the indicated level group; that is, $\left[\mathrm{U}(1,1)\left(\mathcal{O}_{F}\right): \mathrm{U}_{\mathcal{D}}\right] \cdot\langle$,$\rangle , and so on.$ We sometimes write $Z \subset \mathrm{GL}_{2}$ for the central subgroup.

The unitary similitude group defined in this subsection is not the one used to construct Shimura varieties. The ones usually used to define Shimura varieties consist of elements whose similitude factors are rational. Our groups here are slightly larger. The reason for this definition is that they are more convenient for constructing various Eisenstein series, since the representation theory is easier and the Hasse principle holds. We restrict the various automorphic forms constructed to the smaller similitude group to get what we want. Let $G=G_{n}=\mathrm{GU}(n, n)$ be 
the unitary similitude group associated to the pairing

$$
w=w_{n}=\left(\begin{array}{c}
1_{n} \\
-1_{n}
\end{array}\right)
$$

on $\mathcal{K}^{2 n}$. We define algebraic groups $G:=\mathrm{GU}(n, n)$ and $\mathrm{U}_{n}=\mathrm{U}(n, n)$ as follows: for any $F$-algebra $R$, the $R$ points are

$$
G(R)=\mathrm{GU}(n, n)(R):=\left\{g \in \mathrm{GL}_{2 n}\left(\mathcal{K} \otimes_{F} R\right) \mid g w g^{*}=\mu(g) w, \mu(g) \in R^{\times}\right\}
$$

$\left(\mu: \mathrm{GU}(n, n) \rightarrow \mathbb{G}_{m}\right.$ is called the similitude character) and

$$
\mathrm{U}(n, n)(R):=\{g \in \mathrm{GU}(n, n)(R) \mid \mu(g)=1\} .
$$

We define $Q=Q_{n}$ to be the Siegel parabolic subgroup of $G$ consisting of block matrices of the form $\left(\begin{array}{ll}A & B \\ C & D\end{array}\right)$ such that $C=0$. Let $P$ be the Klingen parabolic subgroup of $\mathrm{GU}(2,2)$ which consists of matrices of the form $\left(\begin{array}{l}* * * * \\ * * * * * \\ * \\ * * *\end{array}\right)$ and $M_{P}$ its Levi subgroup. For $g \in \mathrm{GU}(1,1), x \in \operatorname{Res}_{\mathcal{K} / \mathcal{F}} \mathbb{G}_{m}$, we write $m(g$,

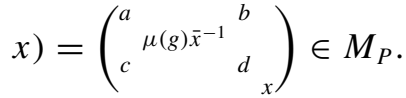

For $v$ a finite prime of $F$, as in [44, Section 8], we define the level group $K_{r, t} \subset$ $\mathrm{GU}(2,2)\left(F_{v}\right)$ for $r, t>0$ as follows: for $Q$ and $P$ being the Siegel and Klingen parabolic respectively,

$$
K_{r, t}=K_{Q, v}\left(\lambda^{r}\right) \cap w_{2}^{\prime} K_{P}\left(\lambda^{t}\right) w_{2}^{\prime},
$$

where $K_{Q, v}\left(\varpi_{v}^{r}\right)$ means the matrices which are in $Q\left(\mathcal{O}_{F, v}\right)$ modulo $\varpi_{v}^{r}$ and

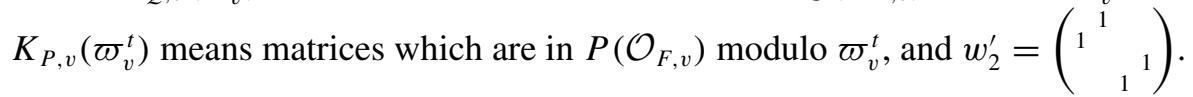
We usually denote $\mathfrak{h}$ as the Poincare upper half plane. Write $\eta$ for the matrix $\left(-1{ }^{1}\right)$.

For any domain $A$ we usually write $F_{A}$ for the fraction field of $A$.

We sometimes write $\epsilon$ for the cyclotomic character and $\omega$ for the Teichmuller character.

2.2. Hermitian half space and automorphic forms. For any finite place $v$ of $F$ let $K_{v}=\mathrm{GU}\left(\mathcal{O}_{F, v}\right)$. Let

$$
\mathbf{H}_{n}:=\left\{Z \in M_{n}(\mathbb{C}):-i\left(Z-{ }^{t} \bar{Z}\right)>0\right\} .
$$

(Note that $\mathbf{H}_{1}$ is the usual upper half plane.) 
Let $Z \in \mathbf{H}_{n}^{\Sigma_{\infty}}$. Write $\alpha=\left(\begin{array}{cc}A & B \\ C & D\end{array}\right) \in G\left(F_{\infty}\right)$ with $A, B, C, D(n \times n)$ block matrices. Let $\mu_{\alpha}(Z):=C Z+D, \kappa_{\alpha}(Z):=\bar{C}^{t} Z+\bar{D}$. We define the automorphy factor:

$$
J(\alpha, Z):=\left(\mu_{\alpha}(Z), \kappa_{\alpha}(Z)\right) .
$$

Let $G\left(F_{\infty}\right)^{+}=\{g \in G(\mathbb{R}), \mu(g) \gg 0\}$, where $\gg 0$ means positive at all Archimedean places. Then $G\left(F_{\infty}\right)^{+}$acts on $\mathbf{H}_{n}^{\Sigma_{\infty}}$ by

$$
g(Z):=\left(A_{g} Z+B_{g}\right)\left(C_{g} Z+D_{g}\right)^{-1}, \quad g=\left(\begin{array}{cc}
A_{g} & B_{g} \\
C_{g} & D_{g}
\end{array}\right) .
$$

Let $K_{\infty}^{+}=\{g \in \mathrm{U}(\mathbb{R}): g(i)=i\}$ (we write $i$ for the matrix $i 1_{n} \in \mathbf{H}_{n}^{\Sigma_{\infty}}$ ) and $Z_{\infty}$ be the center of $G(\mathbb{R})$. Let $K_{\infty}$ be the group generated by $K_{\infty}^{+}$and $\left({ }^{1_{n}}-1_{n}\right)$. We define $C_{\infty}:=Z_{\infty} K_{\infty}^{+}$. Then $k_{\infty} \mapsto J\left(k_{\infty}, i\right)$ defines a homomorphism from $C_{\infty}$ to $\mathrm{GL}_{n}(\mathbb{C}) \times \mathrm{GL}_{n}(\mathbb{C})$.

DEFINITION 9. A weight $\underline{k}$ is a set of integers $\left(k_{n+1, \sigma}, \ldots, k_{2 n, \sigma} ; k_{n, \sigma}, \ldots, k_{1, \sigma}\right)$ such that $k_{1, \sigma} \geqslant k_{2, \sigma} \geqslant \cdots \geqslant k_{2 n, \sigma}$ and $k_{n, \sigma} \geqslant k_{n+1, \sigma}+2 n$.

A weight $\underline{k}$ defines an algebraic representation of $\mathrm{GL}_{n}(\mathbb{C})^{\Sigma_{\infty}} \times \mathrm{GL}_{n}(\mathbb{C})^{\Sigma_{\infty}}$ by

$$
\rho_{\underline{k}}\left(g_{+}, g_{-}\right):=\rho_{\left(k_{n}, \ldots, k_{1}\right)}\left(g_{+}\right) \otimes \rho_{\left(-k_{n+1}, \ldots,-k_{2 n}\right)}\left(g_{-}\right),
$$

where $\rho_{\left(a_{1}, \ldots, a_{n}\right)}$ is the dual of the usual irreducible algebraic representation of $\mathrm{GL}_{n}$ with highest weight $\left(a_{1}, \ldots, a_{n}\right)$. Let $V_{\underline{k}}(\mathbb{C})$ be the representation of $C_{\infty}$ given by

$$
k_{\infty} \rightarrow \rho_{\underline{k}} \circ J\left(k_{\infty}, i\right)
$$

Fix $K$ an open compact subgroup of $G\left(\mathbb{A}_{f}\right)$. We let

$$
S h_{K}(G)=G(F)^{+} \backslash \mathbf{H}_{n}^{\Sigma_{\infty}} \times G\left(\mathbb{A}_{F, f}\right) / K C_{\infty} .
$$

The automorphic sheaf $\omega_{\underline{k}}$ is the sheaf of holomorphic sections of

$$
G(F)^{+} \backslash \mathbf{H}_{n}^{\Sigma_{\infty}} \times G\left(\mathbb{A}_{F, f}\right) \times V_{\underline{k}}(\mathbb{C}) / K C_{\infty} \rightarrow G(\mathbb{Q})^{+} \backslash \mathbf{H}_{n}^{\Sigma_{\infty}} \times G\left(\mathbb{A}_{F, f}\right) / K C_{\infty} .
$$

One can also define these Shimura varieties and automorphic sheaves in terms of moduli of abelian varieties. We omit these here.

The global sections of $\omega_{\underline{k}}$ are called the space of modular forms. These are functions

$$
f: \mathbf{H}_{n}^{\Sigma_{\infty}} \times G\left(\mathbb{A}_{F, f}\right) \rightarrow V_{\underline{k}}(\mathbb{C})
$$


that are holomorphic in the first variable, fixed by some open compact subgroup $K$ of the second variable, and such that

$$
\mu(\gamma)^{\left(k_{1}+\cdots+k_{2 n}\right) / 2} \rho_{\underline{k}}(J(\gamma, Z))^{-1} f(\gamma(Z), g)=f(Z, g)
$$

for all $\gamma \in g K^{-1} \cap G^{+}(\mathbb{Q})$. Also, when $n=1$ we require a moderate growth condition.

REMARK 10. We will be mainly interested in the scalar-valued forms. In this case $V_{\underline{k}}(\mathbb{C})$ is one dimensional of weight $\underline{k}_{\sigma}=(0, \ldots, 0 ; \kappa, \ldots, \kappa)$ for any $\sigma$ and some integer $\kappa \geqslant 2$.

\subsection{Hilbert modular forms.}

2.3.1. Hilbert modular forms. We set up the basic notions of Hilbert modular forms, following [15] with minor modifications. Let $I$ be the set of all field embeddings of $F$ into $\overline{\mathbb{Q}}$. We may regard $I$ as the set of infinite places of $F$ via $\iota_{\infty}: \overline{\mathbb{Q}} \hookrightarrow \mathbb{C}$. The weight of a Hilbert modular form over $F$ is a pair of elements $(\kappa, w)$ in the free module $\mathbb{Z}[I]$ generated by $I$ such that $\kappa-2 w$ is parallel. We identify $F_{\infty}=F \otimes_{\mathbb{Q}} \mathbb{R}$ with $\mathbb{R}^{I}$ and embed $F$ into $\mathbb{R}^{I}$ via the diagonal map $a \mapsto\left(a^{\sigma}\right)_{\sigma \in I}$. Then the identity component $G_{\infty}^{+}$of $\mathrm{GL}_{2}\left(F_{\infty}\right)$ naturally acts on $\mathscr{L}=\mathfrak{h}^{I}$ with $\mathfrak{h}$ the Poincare half plane. We write $C_{\infty}^{+}$for the stabilizer in $G_{\infty}^{+}$of the center point $z_{0}=(\sqrt{-1}, \sqrt{-1}, \ldots, \sqrt{-1}) \in \mathscr{L}$. Then, for each open compact subgroup $U$ of $\mathrm{GL}_{2}\left(\mathbb{A}_{F_{f}}\right)$, we denote by $M_{\kappa, w}(U ; \mathbb{C})$ the space of holomorphic modular forms of weight $(\kappa, w)$ with respect to $S$ (see [15] for more detail), namely $M_{\kappa, w}(U ; \mathbb{C})$ is the space of smooth functions $f: \mathrm{GL}_{2}\left(\mathbb{A}_{F}\right) \rightarrow \mathbb{C}$ satisfying the automorphic condition

$$
f(\alpha x u)=f(x) j_{\kappa, w}\left(u_{\infty}, z_{0}\right)^{-1} \quad \text { for } \alpha \in \mathrm{GL}_{2}(F) \text { and } u \in U C_{\infty}^{+},
$$

where $j_{\kappa, w}\left(\left(\begin{array}{ll}a & b \\ c & d\end{array}\right), z\right)=(a d-b c)^{-w}(c z+d)^{\kappa}$ for $\left(\begin{array}{ll}a & b \\ c & d\end{array}\right) \in \mathrm{GL}_{2}\left(F_{\infty}\right)$ and $z \in \mathscr{L}$ and such that for any $g_{f} \in \mathrm{GL}_{2}\left(\mathbb{A}_{f}\right)$ the associated classical form defined by $f_{c l}\left(z, g_{f}\right):=f(g) \cdot j_{\kappa, w}\left(g_{\infty}, z_{0}\right)$ for $g=\left(g_{0}, g_{\infty}\right)$ such that $g_{\infty} \cdot z_{0}=z$ is holomorphic on the symmetric domain and at all cusps. We write $S_{\kappa, w}(U ; \mathbb{C})$ for the subspace of $M_{\kappa, w}(U ; \mathbb{C})$ consisting of cusp forms. Here we have used the convention that $c^{s}=\prod_{\sigma \in I} c_{\sigma}^{s_{\sigma}}$ for $c=\left(c_{\sigma}\right)_{\sigma \in I} \in \mathbb{C}^{I}$ and $s=\sum s_{\sigma} \sigma \in \mathbb{C}[I]$. Setting $t=\sum_{\sigma} \sigma$, we sometimes use another pair $(n, v)$ to denote the weight, for $n=\kappa-2 t$ and $v=t-w$. Each automorphic representation $\pi$ spanned by forms in $S_{\kappa, w}(U ; \mathbb{C})$ has central character $|\cdot|_{\mathbb{A}}^{-m}$ up to a finite-order character. The twist $\pi^{u}=\pi \otimes|\cdot|_{\mathbb{A}}^{m / 2}$ is called the unitarization of $\pi$. 
Let $h$ be the narrow class number of $F$, and decompose

$$
\mathbb{A}_{F}^{\times}=\bigsqcup_{i=1}^{h} F^{\times} a_{i}\left(\hat{\mathcal{O}}_{F}\right)^{\times} F_{\infty+}^{\times} \quad \text { with } a_{i} \in \mathbb{A}_{F, f}^{\times},
$$

where $F_{\infty+}^{\times}$is the set of totally positive elements. Then by strong approximation

$$
G\left(\mathbb{A}_{F}\right)=\bigcup_{i=1}^{h} \mathrm{GL}_{2}(F) t_{i} U_{0}(N) G_{\infty+} \quad \text { for } t_{i}=\left(\begin{array}{cc}
a_{i}^{-1} & 0 \\
0 & 1
\end{array}\right),
$$

where for any ideal $N$ of $\mathcal{O}_{F}$ let $U_{0}(N)\left(U_{1}(N)\right)$ be the open compact subgroup of $\mathrm{GL}_{2}\left(\hat{\mathcal{O}}_{F}\right)$ whose image modulo $N$ is inside $B\left(\hat{\mathcal{O}}_{F}\right)\left(N\left(\hat{\mathcal{O}}_{F}\right)\right)$. Let $T$ be the diagonal torus of $\mathrm{GL}_{2}$ and $\varepsilon$ be a neben character of $T\left(\hat{\mathcal{O}}_{F}^{\times}\right)$whose conductor contains $(N)$. Let $M_{\kappa, w}\left(U_{0}(N), \varepsilon ; \mathbb{C}\right)$ be the space of Hilbert modular forms of weight $(\kappa, w)$ with level group $U_{0}(N)$ and nebentypus $\varepsilon$. Any automorphic form in the space $M_{\kappa, w}\left(U_{0}(N), \varepsilon ; \mathbb{C}\right)$ is determined by its restriction to the connected components of the $t_{i}$ in $\mathrm{GL}_{2}(F) \backslash \mathrm{GL}_{2}\left(\mathbb{A}_{F}\right) / U_{0}(N) G_{\infty+}$. So we identify the above space with the space of $h$-tuples: $\left\{f_{i}\right\}$, where the $f_{i}$ are forms in $M_{\kappa, w}\left(\Gamma_{i} \backslash \mathfrak{h}^{\Sigma_{\infty}}\right.$, C) for $\Gamma_{i}:=t_{i} U_{0}(N) t_{i}^{-1} \cap \mathrm{GL}_{2}(F)$ with $f_{i}\left(g_{\infty}\right):=f\left(g_{\infty} t_{i}\right)$. Each $f_{i}$ has a $q$ expansion:

$$
f_{i}(z)=a\left(0, f_{i}\right)+\sum_{0 \ll \xi \in F^{\times}} a\left(\xi, f_{i}\right) e_{F}(\xi z) .
$$

More generally we have the $q$-expansions for Hilbert modular forms at $y \in \mathrm{GL}_{2}\left(\mathbb{A}_{F, f}\right)$. More precisely, each $f \in M_{\kappa, w}(U ; \mathbb{C})$ has a Fourier expansion at $y$ of the following type:

$$
f\left(\left(\begin{array}{c}
y_{\infty} x_{\infty} \\
1
\end{array}\right) y\right)=y_{\infty}^{w} \cdot\left(a_{0}(y, f)+\sum_{0 \ll \xi \in F^{\times}} a(y \xi, f) q^{\xi}\right)
$$

with $q^{\xi}=e_{F}\left(2 \pi i \xi_{\infty}\left(x_{\infty}+i y_{\infty}\right)\right)=e\left(2 \pi i \sum_{\sigma \mid \infty} \xi_{\sigma}\left(x_{\sigma}+i y_{\sigma}\right)\right)$. For any $t \in \mathbb{A}_{F}^{\times}$ we define $a(t, \xi, f)$ to be $a(\operatorname{diag}(t, 1), \xi, f)$.

2.3.2. Hida families. First of all let us define the weight space for Hilbert modular Hida families. We fix an even number $\kappa_{0} \geqslant 2$ throughout the paper. For $A$ the integer ring of some finite extension of $\mathbb{Q}_{p}$, let $\Lambda_{W, A}^{\prime}=\Lambda_{W}^{\prime}$ be the $A$-algebra parameterizing continuous characters of $T\left(1+p \mathcal{O}_{F, p}\right)(T$ is the diagonal torus of $\left.\mathrm{GL}_{2}\right)$. This is a power series ring of $2 d$ variables. A point $\phi \in \operatorname{Spec}\left(\Lambda_{W}^{\prime}\right)$ is called arithmetic if, for any $a, b \in 1+\mathcal{O}_{F, p}, \phi\left(\left({ }^{a}{ }_{1}\right)\right)$ is a $p$-power root of unity and $\phi\left(\left({ }^{1}{ }_{b}\right)\right) \mapsto \mathrm{Nm}_{F_{v} / \mathbb{Q}_{p}}(b)^{\kappa_{\phi}-\kappa_{0}}$ times a $p$-power root of unity, where $\kappa_{\phi} \geqslant 2$ is some 
integer. (This means we only consider Hilbert modular forms of parallel weight, which is already enough for constructing the whole Hida family by moving the nebentypus only.)

Let $Z \subset \mathrm{GL}_{2}$ be the center. Define $\Lambda_{W}=\Lambda_{W, A}$ such that $\operatorname{Spec} \Lambda_{W}$ is the closed subspace of $\operatorname{Spec} \Lambda_{W}^{\prime}$ defined as the Zariski closure of the arithmetic points such that $\left.\phi\right|_{Z\left(1+\mathcal{O}_{F, p}\right)}$ factors through $\mathrm{Nm}_{\mathcal{O}_{F, p} / \mathbb{Z}_{p}}$. It is naturally a power series ring with $d+1$ variables. We only consider this weight space for simplicity. In fact, if the Leopoldt conjecture is true, then this is the whole weight space for Hida families of Hilbert modular forms. We define the parallel weight space $\Lambda_{W}^{\text {para }}$ whose spectrum is the Zariski closure in $\operatorname{Spec} \Lambda_{W}$ of points such that the composition of $1+p \mathcal{O}_{F, p} \rightarrow T\left(1+\mathcal{O}_{F, p}\right), x \mapsto\left({ }_{1}^{x}{ }_{1}\right)$ with $\phi$ factors through

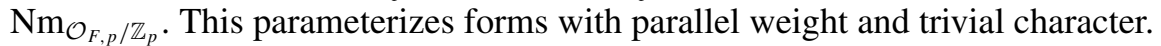

Now we define the nebentypus associated to $\phi$ : the $\phi$ determines a character of $T\left(1+p \mathcal{O}_{F, p}\right)$. We extend these to be characters on $\mathcal{O}_{v}^{\times}$by requiring them to be trivial on the torsion part of $\mathcal{O}_{F, p}^{\times}$. Define

$$
\varepsilon_{\phi, v}\left(\left(\begin{array}{c}
a \\
b
\end{array}\right)\right)=\phi(\operatorname{diag}(a, b)) \omega^{\kappa_{\phi}-\kappa_{0}}(b)
$$

for $a, b \in \mathcal{O}_{F, p}^{\times}$(recall that $\omega$ is the Teichmuller character). It is well known that in the Hilbert modular form case there is a nearly ordinary idempotent $e=\lim _{n \rightarrow \infty}\left(\prod_{v \mid p} U_{v}\right)^{n !}$ defined by Hida, where $U_{v}$ is the usually ' $U_{p}$ ' operator associated to $\left(\begin{array}{ll}p & \\ & 1\end{array}\right)$ at $v$.

DEFINITION 11. A Hilbert modular form $f$ is called nearly ordinary if $e f=f$. We define $M_{\kappa, w}^{\text {ord }}(U ; \mathbb{C})$ and $S_{\kappa, w}^{\text {ord }}(U ; \mathbb{C})$ to be the space of nearly ordinary modular forms and the space of cusp forms with level group $U$.

REMARK 12. We say that $f$ is of nebentypus $\varepsilon_{\phi}$ if

$$
f(g t)=f(g) \varepsilon_{\phi}(t)
$$

for $t \in T\left(\mathcal{O}_{F, p}\right)$. Suppose that $f$ is a nearly ordinary unitary eigenform of weight $\left(\kappa_{\phi}, \kappa_{\phi} / 2\right)$ and nebentypus $\varepsilon_{\phi}$. Then we can assume that for each $v \mid p$ the $v$ component of the automorphic representation $\pi_{f}$ of $f$ is $\pi\left(\mu_{1, v}, \mu_{2, v}\right)$, where $\operatorname{val}_{p} \mu_{1, v}(p)=-\left(\kappa_{\phi}-1\right) / 2, \operatorname{val}_{p} \mu_{2, v}(p)=\left(\kappa_{\phi}-1\right) / 2$ and $\mu_{1, v}, \mu_{2, v}$ have the same restriction to $\mathcal{O}_{F, v}^{\times}$as $\varepsilon_{1, \phi, v}^{\prime}$ and $\varepsilon_{2, \phi, v}^{\prime} \omega^{\kappa_{\phi}-2}$, respectively.

Let $\mathbb{I}$ be a finite integral extension of either $\Lambda_{W}$ or $\Lambda_{W}^{\text {para }}$ which is a local normal domain. 
DEFINITION 13. Let $M$ be an ideal of $F$ prime to $p$. An $\mathbb{I}$-adic nearly ordinary cusp form $\mathbf{f}$ of level $U_{0}(M)$ or $U_{1}(M)$ and nebentypus $\varepsilon$ is a set of elements of $\mathbb{I}$ :

$$
\left\{c_{i}(\xi, \mathbb{I}) \in \mathbb{I} \text { for } \xi \in F^{\times}, c_{i}(0, \mathbb{I}) \in \mathbb{I} \text { for } i=1, \ldots, h\right\}
$$

with the property that for a Zariski dense set of primes $\phi \in$ SpecII which map to arithmetic points in $\operatorname{Spec}\left(\Lambda_{W}\right)$, the specializations $\phi\left(c_{i}(\xi, \mathbb{I})\right)$ are the $q$ expansions $a_{0}\left(t_{i}, \mathbf{f}_{\phi}\right)$ or $a\left(t_{i} \xi, \mathbf{f}_{\phi}\right)$ (see (2.1)) of nearly ordinary cusp forms $\mathbf{f}_{\phi}$ of weight $\left(\kappa_{\phi}, \kappa_{\phi} / 2\right)$, prime to $p$ level $M$ and nebentypus $\varepsilon_{\phi}$ at primes dividing $p$.

We discuss the analog of the 'normalized' cuspidal eigenform on $\mathrm{GL}_{2}\left(\mathbb{A}_{\mathbb{Q}}\right)$. In the $\mathbb{Q}$ case we take the form with $a_{1}=1$ to be normalized. In the totally real case we require the normalized cuspidal eigenform $f$ to have $a\left(\mathfrak{d}^{-1}, f\right)=1$.

2.3.3. Galois representations of Hilbert modular forms. Let $A$ be a finite extension of $\mathbb{Q}_{p}$. One can also define the space of Hilbert modular forms $M_{\kappa, w}\left(U_{0}(N), \varepsilon, A\right)$ and the corresponding cuspidal spaces $S_{\kappa, w}\left(U_{0}(N), \varepsilon, A\right)$. Let

$$
f \in S_{\kappa, w}\left(U_{0}(N), \varepsilon, A\right)
$$

with coefficient ring $A, \kappa \geqslant 2$, nebentypus $\varepsilon$. Recall that we fix $L \subset \overline{\mathbb{Q}}_{p}$ to be a finite extension of $\mathbb{Q}_{p}$ containing all the Fourier coefficients of $f$. For $\mathcal{O}_{L}$ be the integer ring of $L$ and $\mathbb{F}$ its residue field. Then we have a continuous semisimple two-dimensional Galois representation [46] $\left(\rho_{f}, V_{f}\right): \rho_{f}: G_{\mathbb{Q}} \rightarrow \mathrm{GL}_{2}\left(V_{f}\right)$, characterized by being unramified at primes $v \nmid p$ where $\pi_{v}$ is unramified and satisfying

$$
\operatorname{tr} \rho_{f}\left(\operatorname{Frob}_{v}\right)=a(v, f),
$$

where $a(v, f)$ is the Hecke eigenvalue of $f$ under the Hecke operator $T_{v}$ (recall that this is associated to $\left({ }^{\sigma_{v}} 1\right)$, where $\varpi_{v}$ is a uniformizer at $v$ ). Furthermore, if $f$ is nearly ordinary at all primes dividing $p$, then we have the following description of $\rho_{f}$ restricted to the decomposition groups for all primes $v$ dividing $p$ :

$$
\left.\rho_{f}\right|_{G_{F_{v}}} \simeq\left(\begin{array}{cc}
\sigma_{\mu_{1, v}} & * \\
& \sigma_{\mu_{2, v}}
\end{array}\right) .
$$

Here $\sigma$ is the local reciprocity map via local class field theory (we use the geometric Frobenius normalization) and $\pi_{v} \simeq \pi\left(\mu_{1, v}, \mu_{2, v}\right)$, where $\mu_{1, v}(p)$ has smaller $p$-valuation than $\mu_{2, v}(p)$.

Therefore for each $v \mid p$ we have a one-dimensional subspace $V_{f, v}^{+} \subset V_{f}$ such that the action of $G_{v}$ on $V_{f, v}^{+}$is given by the character $\sigma_{\mu_{1, v}}$ and $G_{v}$ acts on the quotient $V_{f, v}^{-}:=V_{f, v} / V_{f, v}^{+}$by $\sigma_{\mu_{2, v}}$. 
Assume that $\rho_{f}$ is residually irreducible. We choose a Galois stable $\mathcal{O}_{L}$ lattice $T_{f}$ of $V_{f}$ which is unique after scaling. Let $T_{f}^{+}=V_{f}^{+} \cap T_{f}$ and $T_{f}^{-}=T_{f} / T_{f}^{+}$.

Similarly, let $\mathbf{f}$ be a Hida family of eigenforms with coefficient ring $\mathbb{I}$. Suppose that the residual Galois representation $\bar{\rho}_{f_{\phi}}$ for some member $f_{\phi}$ of this family is irreducible ((irred) is defined in the introduction). Then it is well known that we have a Galois representation

$$
\rho_{\mathbf{f}}: G_{F} \rightarrow \mathrm{GL}_{2}(\mathbb{I}),
$$

and we denote the representation space (a free rank-two module over $\mathbb{I}$ ) by $T_{\mathrm{f}}$ and we have similarly $T_{\mathrm{f}}^{+}$and $T_{\mathrm{f}}^{-}$. This Galois representation is characterized by

$$
\operatorname{Tr} \rho_{\mathbf{f}}\left(\sigma_{\mathfrak{l}}\right)=T_{\mathfrak{l}}(\mathbf{f})
$$

for every geometric Frobenius $\sigma_{\mathfrak{l}}$ at $\mathfrak{l} \nmid p N\left(T_{\mathfrak{l}}(\mathbf{f})\right.$ is the Hecke eigenvalue of $T_{\mathfrak{l}}$ acting on $\mathbf{f}$ ). In fact one can construct a pseudorepresentation by patching the Galois representation for each arithmetic specializations of $\mathbf{f}$ and then produce an actual Galois representation for $\mathbf{f}$ under assumption (irred).

2.4. Selmer groups. We recall the notion of $\Sigma$-imprimitive Selmer groups, emphasizing the case of Hilbert modular case, following [44, 3.1] with some modifications. Let $F$ be a totally real number field as before. Let $T$ be a free module of finite rank over a profinite $\mathbb{Z}_{p}$-algebra $A$, and assume that $T$ is equipped with a continuous action of $G_{F}$. Denote by $A^{*}$ the Pontryagin dual of $A$. Assume furthermore that for each place $v \mid p$ of $F$ we are given a $G_{v}$-stable rank-one free $A$-direct summand $T_{v} \subset T$. For any finite set of primes $\Sigma$ we denote by $\operatorname{Sel}_{F}^{\Sigma}\left(T,\left(T_{v}\right)_{v \mid p}\right)$ the kernel of the restriction map:

$$
H^{1}\left(F, T \otimes_{A} A^{*}\right) \rightarrow \prod_{v \notin \Sigma, v \nmid p} H^{1}\left(I_{v}, T \otimes_{A} A^{*}\right) \times \prod_{v \mid p} H^{1}\left(I_{v}, T / T_{v} \otimes_{A} A^{*}\right) .
$$

We now assume that $\Sigma$ contains all primes at which $T$ is ramified. We put

$$
X_{F}^{\Sigma}\left(T,\left(T_{v}\right)_{v \mid p}\right):=\operatorname{Hom}_{A}\left(\operatorname{Sel}_{F}^{\Sigma}\left(T,\left(T_{v}\right)_{v \mid p}\right), A^{*}\right) .
$$

If $E / F$ is a finite extension, we put $\operatorname{Sel}_{E}^{\Sigma}(T):=\operatorname{Sel}_{E}^{\Sigma_{E}}\left(T,\left(T_{w}\right)_{w \mid p}\right)$ and $X_{E}^{\Sigma}(T):=$ $X_{E}^{\Sigma_{E}}\left(T,\left(T_{w}\right)_{w \mid p}\right)$, where $\Sigma_{E}$ is the set of places of $E$ over those in $\Sigma$, and if $w|v| p$ then $T_{w}=g_{w} T_{v}$ for $g_{w} \in G_{F}$ such that $g_{w}^{-1} G_{E, w} g_{w} \subseteq G_{F, v}$. If $E / F$ is infinite we set $\operatorname{Sel}_{E}^{\Sigma}(T)=\lim _{E \subseteq F^{\prime} \subseteq E} \operatorname{Sel}_{F^{\prime}}^{\Sigma}(T)$ and $X_{E}^{\Sigma}(T)=\lim _{F \subseteq F^{\prime} \subseteq E} X_{F^{\prime}}^{\Sigma}(T)$, where $F^{\prime}$ runs over the finite extensions of $F$ contained in $E$.

There is an action of complex conjugation $c$ on the Selmer groups of $\mathcal{K}$. We have the following lemma as in [44, Lemma 3.1]. (Recall that we have assumed that $p \neq 2$.) 
LEMMA 14. There is a decomposition

$$
\operatorname{Sel}_{\mathcal{K}}^{\Sigma}(T)=\operatorname{Sel}_{\mathcal{K}}^{\Sigma}(T)^{+} \oplus \operatorname{Sel}_{\mathcal{K}}^{\Sigma}(T)^{-}
$$

according to the \pm 1 eigenspaces of the action by c. Also, restriction induces isomorphisms

$$
\operatorname{Sel}_{F}^{\Sigma}(T) \rightarrow \operatorname{Sel}_{\mathcal{K}}^{\Sigma_{\mathcal{K}}}(T)^{+} \quad \operatorname{Sel}_{F}^{\Sigma}\left(T \otimes \chi_{\mathcal{K}}\right) \rightarrow \operatorname{Sel}_{\mathcal{K}}^{\Sigma_{\mathcal{K}}}(T)^{-}
$$

\subsection{Iwasawa theory of Selmer groups.}

2.5.1. Control of Selmer groups. Before formulating the main conjecture we first define the characteristic ideals and the Fitting ideals. We let $A$ be a Noetherian ring. We write $\operatorname{Fitt}_{A}(X)$ for the Fitting ideal in $A$ of a finitely generated $A$-module $X$. This is the ideal generated by the determinant of the $r \times r$ minors of the matrix giving the first arrow in a given presentation of $X$ :

$$
A^{s} \rightarrow A^{r} \rightarrow X \rightarrow 0 .
$$

If $X$ is not a torsion $A$-module then $\operatorname{Fitt}(X)=0$.

Fitting ideals behave well with respect to base change. For $I \subset A$ an ideal, then

$$
\operatorname{Fitt}_{A / I}(X / I X)=\operatorname{Fitt}_{A}(X) \bmod I .
$$

Now suppose that $A$ is a Krull domain (a domain which is Noetherian and normal). Then the characteristic ideal is defined by

$\operatorname{char}_{A}(X):=\left\{x \in A: \operatorname{ord}_{Q}(x) \geqslant \operatorname{length}_{Q}(X)\right.$ for any $Q$ a height-one prime of $\left.A\right\}$.

Again if $X$ is not torsion then we define $\operatorname{char}_{A}(X)=0$.

We recall some results in $[44,3.2]$ with minor modifications to the totally real situation. These will be used in proving the main theorem in the last section. In this (and only in this) subsubsection we let $A$ be any profinite $\mathbb{Z}_{p}$ algebra and $\mathfrak{a}$ be an ideal of $A$. Let $T$ be a free $A$-module equipped with a continuous $G_{F}$-action and let $T^{*}:=T \otimes_{A} A^{*}$. Let $\mathfrak{a} \subset A$ be an ideal. It is noted in $[44,3.2 .5]$ that there is a canonical map

$$
\operatorname{Sel}_{F}^{\Sigma}(T / \mathfrak{a} T) \rightarrow \operatorname{Sel}_{F}^{\Sigma}(T)[\mathfrak{a}] .
$$

Here $[\mathfrak{a}]$ on the right-hand side means the $\mathfrak{a}$-torsion part.

Proposition 15. Suppose that there is no nontrivial A-subquotient of $T^{*}$ on which $G_{\mathcal{K}_{\infty}^{+}}$acts trivially. Suppose also that for any prime $\mathfrak{p} \mid p$ of $F$ the action of $I_{\mathfrak{p}}$ on $T / T_{\mathfrak{p}}$ factors through the image of $I_{\mathfrak{p}}$ in $\Gamma_{F}$ and that $\Sigma \cup\{p\}$ contains all 
primes at which $T$ is ramified. Let $\tilde{F}=F_{\infty}, \mathcal{K}_{\infty}^{+}$. Then the above map induces isomorphisms

$$
\operatorname{Sel}_{\tilde{F}}^{\Sigma}(T / \mathfrak{a} T) \simeq \operatorname{Sel}_{\tilde{F}}^{\Sigma}(T)[\mathfrak{a}]
$$

and

$$
X_{\tilde{F}}^{\Sigma}(T) \simeq X_{\tilde{F}}^{\Sigma}(T) / \mathfrak{a} X_{\tilde{F}}^{\Sigma}(T)
$$

Proof. The proof is the same as that of [44, Proposition 3.7].

Descent from $\mathcal{K}_{\infty}$ to $\mathcal{K}_{\infty}^{+}$. We have the following immediate corollaries of the above proposition (see [44, Corollary 3.8, Proposition 3.9]).

COROLLARY 16. We assume that the hypotheses of the above proposition hold. If $\tilde{F}$ is $F_{\infty}$ or $\mathcal{K}_{\infty}^{+}$then

$$
\operatorname{Fitt}_{\tilde{F}, A / \mathfrak{a}}^{\Sigma}(T / \mathfrak{a} T)=\operatorname{Fitt}_{\tilde{F}, A}^{\Sigma}(T) \bmod \mathfrak{a} .
$$

COROLlary 17. Let $I^{-}$be the kernel of the natural map $\Lambda_{\mathcal{K}} \rightarrow \Lambda_{\mathcal{K}}^{+}$. Then, under the hypotheses of the above proposition, we have an isomorphism

$$
X_{\mathcal{K}_{\infty}}^{\Sigma}(T) / I^{-} X_{\mathcal{K}_{\infty}}^{\Sigma}(T) \stackrel{\sim}{\rightarrow} X_{\mathcal{K}_{\infty}^{+}}^{\Sigma}(T)
$$

of $\Lambda_{\mathcal{K}, A^{-}}^{+}$modules.

From $\mathcal{K}_{\infty}^{+}$and $F_{\infty}$ to $\mathcal{K}$ and $F$. Let $\left(T, T_{v}(v \mid p)\right)$ be as above. Let $\phi$ be a algebra homomorphism $\Lambda_{F} \rightarrow \mathbb{C}_{p}$ and $I_{\phi}$ be its kernel.

Proposition 18. Let $\left(T^{\prime},\left.T_{v}^{\prime}\right|_{v \mid p}\right)$ be $\left(T,\left.T_{v}\right|_{v \mid p}\right)$ twisted by $\phi \circ \boldsymbol{\varepsilon}_{F}$. Suppose that there is no nontrivial A-subquotient of $T^{*}$ on which $G_{F}$ acts trivially. Assume that the following hold.

(i) $\Sigma \cup\{$ primes above $p\}$ contains all primes at which $T$ is ramified.

(ii) For any $v \mid p,\left(H^{0}\left(I_{v}, T / T_{v} \otimes_{A} \Lambda_{F, A}^{*}\left(\varepsilon_{F}^{-1}\right)\right) \otimes_{\Lambda_{F}} \Lambda_{F} / I_{\phi}\right)^{G_{v}}=0$.

Then restriction yields isomorphisms

$$
\operatorname{Sel}_{F}^{\Sigma}\left(T^{\prime}\right) \rightarrow \operatorname{Sel}_{F_{\infty}}^{\Sigma}(T)\left[I_{\phi}\right] \quad \text { and } \operatorname{Sel}_{\mathcal{K}}^{\Sigma}\left(T^{\prime}\right) \rightarrow \operatorname{Sel}_{\mathcal{K}_{\infty}^{+}}^{\Sigma}(T)\left[I_{\phi}\right]
$$

Here we have identified $\Lambda_{\mathcal{K}}^{+}$with $\Lambda_{F}$.

This is only a slight generalization of [44, Proposition 3.10], and the proofs are identical. 
2.5.2. Main conjecture. Let $\mathcal{T}:=\left(T_{\mathbf{f}} \otimes_{\mathbb{I}} \mathbb{I}\left[\left[\Gamma_{\mathcal{K}}\right]\right]\left(\left(\boldsymbol{\varepsilon}_{\mathcal{K}}^{-c}\right) \otimes \epsilon\right)\right)$ and $\mathcal{T}_{v}^{+}:=\left(T_{\mathbf{f}}^{+} \otimes_{\mathbb{I}}\right.$ $\left.\mathbb{I}\left[\left[\Gamma_{\mathcal{K}}\right]\right]\left(\left(\boldsymbol{\varepsilon}_{\mathcal{K}}^{-c}\right) \otimes \epsilon\right)\right)$ for each $v \mid p$. Let

$$
\operatorname{char}_{\mathcal{K}, \mathbf{f}}^{\Sigma} \subset \mathbb{I}\left[\left[\Gamma_{\mathcal{K}}\right]\right]
$$

be the characteristic ideal of the dual Selmer group $X_{\mathcal{K}}^{\Sigma}\left(\mathcal{T},\left.\mathcal{T}_{v}^{+}\right|_{v \mid p}\right)$. Let $\mathcal{L}_{\mathbf{f}, \mathcal{K}}^{\Sigma}$ be the $\Sigma$-imprimitive $p$-adic $L$-function we construct in Theorem 82 with the character $\xi=1$ there.

CONJECTURE 19. The Iwasawa main conjecture states that

$$
\operatorname{char}_{\mathbf{f}, \mathcal{K}}^{\Sigma}=\left(\mathcal{L}_{\mathbf{f}, \mathcal{K}}^{\Sigma}\right)
$$

(We have only focused on the special case when the character is trivial in the main conjecture in the introduction.)

\section{Hida theory for unitary Hilbert modular forms}

3.1. Iwasawa algebras. Recall that $A$ is the integer ring of a finite extension of $\mathbb{Q}_{p}$ and $\mathbb{I}$ a finite extension of $\Lambda_{W}$ which is a normal domain. We let $\mathbb{I}_{\mathcal{K}}:=$ $\mathbb{I}\left[\left[\Gamma_{\mathcal{K}}\right]\right]$ and $\Lambda_{\mathbf{D}}:=\mathbb{I}\left[\left[\Gamma_{\mathcal{K}}^{-} \times \Gamma_{\mathcal{K}}\right]\right]=\mathbb{I}_{\mathcal{K}}\left[\left[\Gamma_{\mathcal{K}}^{-}\right]\right]$. Here we used the notation $\mathbf{D}$, which stands for the Eisenstein datum to be defined in the beginning of Section 7. Let $W$ be the element in $\Lambda_{W, A}$ defined as follows. For any arithmetic point $\phi$, the corresponding character of $T\left(1+\mathcal{O}_{F, p}\right)$ restricting to $Z\left(1+\mathcal{O}_{F, p}\right)$ factors through $\mathrm{Nm}: 1+\mathcal{O}_{F, p} \rightarrow 1+p \mathbb{Z}_{p}$; that is, $\phi^{\prime} \circ \mathrm{Nm}=\phi$ for some $\phi^{\prime}$. We require that $\phi(1+W)=\phi^{\prime}(1+p)$ for all such points $\phi$. (It is easily seen that there is such a $W$ in $\Lambda_{W, A}$.) Let

$$
\begin{aligned}
& \alpha: A\left[\left[\Gamma_{\mathcal{K}}\right]\right] \rightarrow \mathbb{I}_{\mathcal{K}}^{-}, \quad \alpha\left(\gamma^{+}\right)=(1+W)^{1 / 2}(1+p), \quad \gamma_{v}^{-} \rightarrow \gamma_{v}^{-} \\
& \beta: \mathbb{Z}_{p}\left[\left[\Gamma_{\mathcal{K}}\right]\right] \rightarrow \mathbb{Z}_{p}\left[\left[\Gamma_{\mathcal{K}}\right]\right], \quad \beta\left(\gamma^{+}\right)=\gamma^{+}, \quad \beta\left(\gamma_{v}^{-}\right)=\gamma_{v}^{-}
\end{aligned}
$$

for each $v$. We also let $\Lambda:=\Lambda_{W}\left[\left[\Gamma_{\mathcal{K}}^{-} \times \Gamma_{\mathcal{K}}\right]\right]$. Thus $\Lambda_{\mathbf{D}}$ is finite over $\Lambda$.

Definition 20. A $\overline{\mathbb{Q}}_{p}$ point $\phi \in \operatorname{Spec} \Lambda_{\mathbf{D}}$ is called arithmetic if $\left.\phi\right|_{\mathbb{I}}$ is arithmetic with some weight $\kappa_{\phi}$, and $\phi\left(\gamma^{+}\right)=\zeta^{+}$for $\zeta^{+} \in \mu_{p^{\infty}}, \phi\left(\gamma_{v, i}^{-}\right)=\zeta_{v, i}^{-}$for $\zeta_{v}^{-} \in \mu_{p^{\infty}}$, $\phi\left(\gamma_{v, i}^{\prime,-}\right)=\left(x_{v, i}^{\prime,-}\right)^{\kappa_{\phi} / 2} \zeta_{v, i}^{\prime,-}$ with $\left(x_{v, i}^{\prime,-}, 1 / x_{v, i}^{\prime,-}\right)=\operatorname{rec}_{\mathcal{K}}^{-1}\left(\gamma_{v, i}^{\prime,-}\right)$ and $\zeta_{v, i}^{\prime,-} \in \mu_{p^{\infty}}$. We use the convention that $\gamma_{v, i}^{\prime,-}$ are elements in the first $\Gamma_{\mathcal{K}}^{-}$and $\gamma_{v, i}^{-}$are elements in the $\Gamma_{\mathcal{K}}$.

We write $\mathcal{X}_{\mathbf{D}}^{a}$ for the set of arithmetic points. Next let $W_{2}:=\left(1+\mathcal{O}_{F, p}\right)^{4}$ and $\Lambda_{2}$ be the completed group algebra of $W_{2}$ with coefficient ring $A$. We give a 
$\Lambda_{2}$-algebra structure for $\Lambda_{\mathbf{D}}$ by

$$
\left(t_{1}, t_{2}, t_{3}, t_{4}\right) \rightarrow(\alpha \otimes \beta)\left(\operatorname{rec}_{\mathcal{K}_{p}}\left(t_{3} t_{4}, t_{1}^{-1} t_{2}^{-1}\right) \times \operatorname{rec}_{\mathcal{K}_{p}}\left(t_{4}^{-1}, t_{2}\right)\right) \cdot\left(\begin{array}{ll}
t_{1} t_{3}^{-1} & \\
& 1
\end{array}\right) .
$$

In the last term we regard the matrix as an element of $\Lambda_{W, A}$. Here rec means the reciprocity map in class field theory. From this $\Lambda \subset \Lambda_{\mathbf{D}}$ is also a $\Lambda_{2}$-algebra and is in fact a quotient of $\Lambda_{2}$.

REMARK 21. When $F=\mathbb{Q}$, then $\Lambda_{2}=\Lambda$. In general, $\Lambda$ is of lower dimension. In other words we are only considering a subfamily of the whole weight space. This is the weight space on which our Klingen Eisenstein family sits. Only on this weight space we can prove required control results and freeness of the family of nearly ordinary forms (not necessarily cuspidal).

3.2. Igusa tower and $p$-adic automorphic forms. For any $v \mid p, \mathrm{U}(2,2) \simeq$ $\mathrm{GL}_{4}\left(\mathbb{Z}_{p}\right)$ under the projection to the first factor of $\mathcal{K}_{v}=F_{v} \times F_{v}$. (Recall that our convention is that the first factor corresponds to the Archimedean place inside the CM type under $\iota: \mathbb{C} \simeq \mathbb{C}_{p}$.) Define $B$ to be the standard Borel $\left(\begin{array}{cc}\times & \times \\ \times & \times \\ x & \times \\ \times & \times\end{array}\right)$ and $N$ to be the unipotent radical of $B$. Let $I_{0, s}\left(I_{1, s}\right)$ consist of elements in $\mathrm{U}(n, n)\left(\mathbb{Z}_{p}\right)$ which are in $B\left(\mathbb{Z}_{p} / p^{s} \mathbb{Z}_{p}\right)\left(N\left(\mathbb{Z}_{p} / p^{s}\right)\right)$ modulo $p^{s}$ (see $[44,5.3 .6]$ ).

We refer to the definition of Shimura varieties $S(K)$ (over $\mathcal{O}_{(p)}$, the localization at $p$ of the integer ring of the reflex field) for the unitary similitude group and open compact $K \subset G\left(\mathbb{A}_{F, f}\right)$ such that the components of $K$ are $\mathrm{GL}_{4}\left(\mathbb{Z}_{p}\right)$ at all primes above $p$ and the automorphic sheaves $\omega_{\underline{k}}$ and the universal differential sheaf $\omega$ to [29], [16] and [23], respectively. Let $S(K)^{*}$ and $\mathcal{S}(K)$ be the minimal and a fixed toroidal compactification of $S(K)$, again over $\mathcal{O}_{(p)}$. For $0 \leqslant q \leqslant n$ as in [44, Section 5], one defines the genus $q$ cusps, and we let $\mathcal{I}_{S^{*}(K)}^{q}$ be the ideal sheaf of $S^{*}(K)$ of the boundary of genus less than $n-q$. Let $\mathcal{I}_{\mathcal{S}(K)}^{q}$ be the corresponding pullback sheaf on $\mathcal{S}(K)$. Recall that a weight is $\underline{k}=\left\{\underline{k}_{\sigma}\right\}_{\sigma \in \Sigma}$ where $\underline{k}_{\sigma}=\left(k_{s+1, \sigma}, \ldots, k_{r+s, \sigma} ; k_{1, \sigma}, \ldots, k_{s, \sigma}\right)$. We write $M_{\underline{k}}(K, R)$ for the space of automorphic forms with weight $\underline{k}$, level $K$, and coefficient $R$. We write $M_{k}^{0}(K, R)$ for the cuspidal part.

Let $L$ be a finite extension of $\mathbb{Q}_{p}$. Let $\mathcal{I}_{\mathcal{S}}$ be the ideal of the boundary of $\mathcal{S}$. There is a subsection Hass of $\operatorname{det}(\omega)^{\otimes(p-1)} \otimes \overline{\mathbb{F}}_{p}$, called the Hasse invariant. Since $\operatorname{det}(\omega)$ is ample on the minimal compactification $\mathcal{S}^{*}$, one finds $E$, a lifting of Hass $^{m}$ over $\mathcal{O}_{L}$ for sufficiently large $m$. Then $\mathcal{S}^{*}[1 / E]$ is affine. For any positive integer $m$, set $S_{m}:=\mathcal{S}[1 / E] \times \mathcal{O}_{L} / p^{m}$. Let $H=\mathrm{GL}_{2} \times \mathrm{GL}_{2}$. For any integers $s \geqslant m$, we have the Igusa variety $I_{s, m}$ (see [44, Section 6]) which is an etale Galois 
covering of $S_{m}$ with Galois group canonically isomorphic to

$$
\prod_{v \mid p} \mathrm{GL}_{2}\left(\mathcal{O}_{F, v} / p^{s}\right)^{+} \times \mathrm{GL}_{2}\left(\mathcal{O}_{F, v} / p^{s}\right)^{-}=H\left(\prod_{v \mid p} \mathcal{O}_{F, v} / p^{s}\right) .
$$

We put $V_{s, m}^{q}:=\Gamma\left(I_{s, m}, \mathcal{O}_{I_{s, m}} \otimes_{\mathcal{O}_{\mathcal{S}}} \mathcal{I}_{\mathcal{S}}^{q}\right)$. For $j=0,1$, let $I_{j, s}^{H}:=I_{j, s} \cap$ $H\left(\prod_{v \mid p} \mathcal{O}_{v} / p^{s}\right)$, and define

$$
W_{s, m}^{q}:=H^{0}\left(I_{1, s}^{H}, V_{s, m}^{q}\right)
$$

and

$$
\left.\mathcal{W}^{q}:=\underset{m}{\lim } \underset{s}{\lim } W_{s, m}^{q}\right) .
$$

As in [23, Section 2], we define the $p$-adic cusps to be the set of ([g],h) with $[g]$ a cusp (of any genus) and $h \in H\left(\mathcal{O}_{F, p}\right)$. These can be thought of as cusps on the Igusa varieties. For $q=0$ or 1 we also define the space of $p$-adic automorphic forms on $G$ of weight $\underline{k}$ and level $K=K_{p}^{0} K^{p}$ with $p$ divisible coefficients:

$$
V_{\underline{k}}^{q}\left(K, L / \mathcal{O}_{L}\right):=\underset{m}{\lim } \Gamma\left(S_{m}, \omega_{\underline{k}} \otimes_{\mathcal{O}_{\mathcal{S}}} \mathcal{I}_{\mathcal{S}}\right) .
$$

Similarly, if $A$ is an $\mathcal{O}_{L}$-algebra the space of $p$-adic automorphic forms with coefficients in $A$ are defined as the inverse limits:

$$
V_{\underline{k}}(K, A):={\underset{\lim }{m}} \Gamma\left(S_{m},\left(\omega_{\underline{k}} \otimes_{\mathcal{O}_{\mathcal{S}}} \mathcal{I}_{\mathcal{S}}\right) \otimes_{\mathcal{O}_{L}} A\right)
$$

Finally, for any $\underline{a}=\left\{a_{v}\right\}_{v \mid p}$ where each $a_{v} \in\left(\mathbb{F}_{p}^{\times}\right)^{4}$ we define the modules $V_{\underline{a}, \underline{k}}^{q}\left(K, L / \mathcal{O}_{L}\right)$, and so on, to be the corresponding component such that the torsion part of $p$-nebentypus is given by $\underline{a}$, in the same way as $[44,6.2]$.

3.3. Nearly ordinary automorphic forms. Hida defined an idempotent $e_{\text {ord }}$ on the space of $p$-adic automorphic forms (see [16, Section 8.1] for compact unitary Shimura varieties). For more general unitary Shimura varieties, $e_{\text {ord }}$ can be constructed following the same ideas of Hida (see [23, 3.8.3, 4.3] for details; note that the construction of $e=e_{U_{p}}$ in $[23,4.3]$ includes our case). Let $t^{\underline{k}}$ and $\psi_{\underline{k}}$ be similar to those in [44, Section 6.1]. We recall the following important theorem of Hida.

LEMMA 22. For any weight $\underline{k}$, we have canonical isomorphisms

$$
V_{\underline{k}, \text { ord }}^{q}\left(K, \mathbb{Q}_{p} / \mathbb{Z}_{p}\right) \simeq \mathcal{W}_{\text {ord }}^{q}[\underline{k}]:=\left\{w \in \mathcal{W}^{q}: t \cdot w=t^{\underline{k}} w \forall t \in T_{H}\left(\mathbb{Z}_{p}\right)\right\}
$$


and

$$
\begin{aligned}
V_{\underline{k}, \text { ord }}^{q}\left(K^{p} I_{s}, \psi, \mathbb{Q}_{p} / \mathbb{Z}_{p}\right) & \simeq\left(\mathcal{W}^{q} \otimes_{\mathbb{Z}} A\right)\left[\psi_{\underline{k}}\right] \\
& :=\left\{w \in \mathcal{W}^{q} \otimes_{\mathbb{Z}} A: t \cdot w=\psi_{\underline{k}}(t) w \forall t \in T_{H}\left(\mathbb{Z}_{p}\right)\right\}
\end{aligned}
$$

for any $\mathbb{Z}_{p}(\psi)$-algebra $A$.

Proof. The proof is the same as that of [44, Lemma 6.5].

PROPOSITION 23. We have, for any sufficiently regular weight $\underline{k} \geqslant 0$ such that $k_{2}-k_{3}$ is parallel, the canonical base-change morphism

$$
\begin{aligned}
e_{\text {ord }} \cdot \Gamma\left(\mathcal{S}^{*}[1 / E], \pi_{*}\left(\omega_{\underline{k}} \otimes_{\mathcal{O}_{\mathcal{S}}} \pi^{*} \mathcal{I}^{q}\right) \otimes \mathbb{Z} / p^{m} \mathbb{Z}\right) \\
\quad \rightarrow e_{\text {ord }} \cdot \Gamma\left(\mathcal{S}^{*}[1 / E], \pi_{*}\left(\omega_{\underline{k}} \otimes_{\mathcal{O}_{\mathcal{S}}} \pi^{*} \mathcal{I}^{q} \otimes \mathbb{Z} / p^{m} \mathbb{Z}\right)\right)
\end{aligned}
$$

is an isomorphism.

Proof. The proof is a generalization of that of [44, Proposition 6.6]. As in [44] we define $\Gamma_{[g]}:=\mathrm{GL}_{\mathcal{K}}\left(W_{s}\right) \cap g K^{-1}$, and $\Gamma_{[g]}(h) \subset \Gamma_{[g]}$ the stabilizer of $h$ (recall that the action of $\gamma$ is given by $\gamma \cdot h={ }^{t} \gamma h \gamma$ ). Also write $B_{h}$ for the Borel subgroup of $H$ that stabilizes the kernel of $h$ (regarded as a Hermitian form) and $N_{h}$ for its unipotent radical. Let $\tilde{R}_{g, \underline{k} / A}=\prod_{h} H^{0}\left(\Gamma_{[g]}(h), \rho_{\underline{k}}(A)\right)$. Define $\bar{R}_{g, \underline{k} / A}=\prod_{h} \rho_{\underline{k}}^{N_{h}}(A)$. We have $\rho_{\underline{k}}^{\Gamma_{[g]}(h)} \subseteq \rho_{\underline{k}}^{B_{h}}$. We want to prove an analog of [44, Lemma 6.8]. If $p$ is nilpotent in $A$, then we can take representatives of $h$ modulo the action of $\Gamma_{[g]}$ such that either (a) $N_{h}=N_{H}$ or (b) $N_{h}=N_{H}^{-}$, and in case (a) $a\left(h, f \mid e_{\text {ord }}\right)=0$ (see [44]) and in case (b) $a\left(h, f \mid e_{\text {ord }}\right) \subseteq \rho_{\underline{k}}^{N_{H}^{-}}(A)=\rho_{\underline{k}}^{N_{h}}(A)$. Since $K$ is neat, $\Gamma_{[g]}(h) / \Gamma_{[g]}(h) \cap N_{h}(\mathcal{K})$ is embedded into the unit group $\mathcal{O}_{F}^{\times}$. Since $k_{2}-k_{3}$ is parallel, $\Gamma_{[g]}(h) / \Gamma_{[g]}(h) \cap N_{h}(\mathcal{K})$ acts trivially on $\rho_{\underline{k}}^{N_{H}^{-}(A)}$. So we have (see [44, Lemma 6.8])

$$
e_{\text {ord }} \tilde{R}_{[g], \underline{k} / A}=e_{\text {ord }} \bar{R}_{g, \underline{k} / A} .
$$

The proposition follows from this in the same way as [44, Proposition 6.6] (using again the fact that $k_{2}-k_{3}$ is parallel).

The following corollary is immediate from the above proposition (see [44, Corollary 6.7]).

COROLLARY 24. For any sufficiently regular weight $\underline{k}$ such that $k_{2}-k_{3}$ is parallel the module $V_{\underline{k} \text { ord }}^{q}\left(K, \mathbb{Q}_{p} / \mathbb{Z}_{p}\right)$ is divisible. 
3.4. $\Lambda$-adic ordinary automorphic forms. Recall that we have defined the Iwasawa algebra $\Lambda_{2}$. There is an action of it on the space of $p$-adic automorphic forms (see [44, Section 6]). As in [44] we have the following theorem by the above corollary.

TheOREM 25. For $q=1$ or 0 , write $\mathbf{V}_{\text {ord }}^{q}=\operatorname{Hom}_{\text {cont }}\left(e_{\text {ord }} \mathcal{W}, \mathbb{Q}_{p} / \mathbb{Z}_{p}\right) \otimes_{\Lambda_{2}} \Lambda$. Then $\mathbf{V}_{\text {ord }}^{q}$ is free of finite rank over $\Lambda$.

Proof. The proof is similar to that of [44, Theorem 6.11], using Corollary 24 and the following theorem, Theorem 26.

We define the space of $\Lambda$-adic forms to be

$$
\mathcal{M}_{\text {ord }}^{q}\left(K^{p}, \Lambda\right)=\operatorname{Hom}_{\Lambda}\left(\mathbf{V}_{\text {ord }}^{q} \otimes_{\Lambda_{2}} \Lambda, \Lambda\right) .
$$

For any finite $\Lambda$ algebra $A$ we also define the space of $A$-adic forms to be

$$
\mathcal{M}_{\text {ord }}^{q}\left(K^{p}, A\right)=\mathcal{M}_{\text {ord }}^{q}\left(K^{p}, \Lambda\right) \otimes_{\Lambda} A .
$$

Classicality of ordinary forms.

THEOREM 26. Let $\underline{t}=\left(t_{\sigma}\right)_{\sigma}$ be such that $\underline{t}_{\sigma}=(0,0 ; 1,1)$ for any $\sigma$. Assume that $q=0$ or 1 . Let $\underline{k}$ be a sufficiently regular such that $k_{2}-k_{3}$ is parallel. There is a constant $C(\underline{k})>0$ such that

$$
e_{\text {ord }} M_{\underline{k}+\ell(p-1) \underline{t}}\left(K, \mathbb{Q}_{p} / \mathbb{Z}_{p}\right) \hookrightarrow V_{\underline{k}+\ell(p-1) \underline{t}, \text { ord }}^{q}\left(K, \mathbb{Q}_{p} / \mathbb{Z}_{p}\right)
$$

is an isomorphism for all $\ell>C(\underline{k})$.

Proof. The proof is similar to that of [44, Theorem 6.10] and uses Corollary 24. See also [23, Theorem 4.19]. The 'parallel' weight condition is important.

From this theorem we know that there are enough classical forms in our family and thus we can construct families of (pseudo)Galois representations from the classical ones.

Fundamental exact sequence. Consider the embedding $T_{1} \hookrightarrow T_{2}$ given by $\left(t_{1}\right.$, $\left.t_{2}\right) \rightarrow\left(t_{1}, 1, t_{2}, 1\right)$. This gives $\Lambda_{2}$ a $\Lambda_{1}=\mathcal{O}_{L}\left[\left[T_{1}\left(1+\mathbb{Z}_{p}\right)\right]\right]$-algebra structure. The following theorem is a generalization of [44, Theorem 6.15] and follows from the above corollary by noting that $\operatorname{Spec} \Lambda$ is the Zariski closure of the weights such that $k_{2}-k_{3}$ is parallel. 
THEOREM 27. For any $\Lambda$-algebra A there is a short exact sequence $0 \rightarrow \mathcal{M}_{\text {ord }}^{0}\left(K^{p}, A\right) \rightarrow \mathcal{M}_{\text {ord }}^{1}\left(K^{p}, A\right) \rightarrow \oplus_{[g] \in C_{n-1}(K)} \mathcal{M}_{\text {ord }}^{0}\left(K_{n-1, g}^{p}, \Lambda_{1}\right) \otimes_{\Lambda_{1}} A \rightarrow 0$ where the next to last arrow is the Siegel operator (that is, the projection to boundary operator).

To see that the image of ordinary forms on $\mathrm{U}(2,2)$ under Siegel operator are ordinary forms on $\mathrm{U}(1,1)$, we may use the argument of [23, Lemma 4.14]. We consider forms whose level groups at $p$ are $K_{0}(p)$ and allow the weights to vary. By the contraction property of the $e_{\text {ord }}$ operator ([23, Proposition 4.4]) we can thus get a Zariski dense set of arithmetic points. For each cusp $[g]$, by Bruhat decomposition we just need to consider the $p$-adic cusps $([g], w)$ for $w$ a Weyl element of $H\left(\mathcal{O}_{F, p}\right) \simeq \prod_{v \mid p} \mathrm{GL}_{2}\left(\mathbb{Z}_{p}\right) \times \mathrm{GL}_{2}\left(\mathbb{Z}_{p}\right)$. The argument in [23] applying [47, Lemma 4.2] implies that for any nearly ordinary form $\mathbf{F}$ its boundary at ([g], $w$ ) can be nonzero only when $w=\mathrm{id}$, and that the $e_{\text {ord }}$ on $\mathrm{U}(2,2)$ induce the $e_{\text {ord }}$ on $\mathrm{U}(1,1)$ at this $p$-adic cusp. See also [47] for a similar fact for the group Gsp(4).

3.5. $q$-expansions. The $q$-expansion principle will be crucial for our later argument. Similar to [44], for $x$ running through a (finite) set of representatives of $G(F) \backslash G\left(\mathbb{A}_{F, f}\right) / K$ with $x_{p} \in Q\left(\mathcal{O}_{F, p}\right)$, we have that the $\Lambda_{2}$-adic $q$-expansion map

$$
\mathcal{M}_{\text {ord }}^{q}\left(K^{p}, \Lambda\right) \hookrightarrow \oplus_{x} \Lambda\left[\left[q^{S_{x}^{+}}\right]\right]
$$

is injective. Here $S_{x}^{+}$is the set of Hermitian matrices $h$ in $M_{2}(\mathcal{K})$ such that $\operatorname{Tr}_{F / \mathbb{Q}} \operatorname{Tr} h h^{\prime} \in \mathbb{Z}$ for all Hermitian matrices $h^{\prime}$ such that $\left(\begin{array}{c}1 \\ h^{\prime} \\ 1\end{array}\right) \in N_{Q}(F) \cap x K x^{-1}$ and $K$ is the open compact of $G\left(\hat{\mathcal{O}}_{F}\right)$ maximal at primes dividing $p$. This follows from the irreducibility of the Igusa tower for the group $\mathrm{SU}(n, n) \subset \mathrm{U}(n, n)$ (defined as the kernel of det) proved by Hida in [20]. For more details see [44, Theorem 6.3] and the discussion at the end of Section 6.2 there. Let $A$ be a torsionfree $\Lambda$ algebra finite over $\Lambda$, and let $\mathcal{X}$ be a Zariski dense subset of primes $Q$ of $A$ such that $Q \cap \Lambda=P_{\psi_{\underline{k}}}$ for some pair $(\underline{k}, \psi)$ (for the definitions, refer to [44, Section 6]). Let $\mathcal{N}_{\mathcal{X}, \text { ord }}^{q}(A)$ be the set of elements $\left(F_{x}\right)_{x} \in \oplus_{x} A\left[\left[q^{S_{x}^{+}}\right]\right]$such that for each $Q \in \mathcal{X}$ above $P_{\psi_{\underline{k}}}$ the reduction of $\left(F_{x}\right)_{x}$ is the $q$-expansion of some element $f \in V_{\underline{k}, \text { ord }}^{q}\left(K^{p} I_{s}, \psi, A / Q\right)$. Then we have the following.

LEMMA 28. The inclusion

$$
\mathcal{M}_{\text {ord }}^{q}\left(K^{p}, A\right) \hookrightarrow \mathcal{N}_{\mathcal{X}, \text { ord }}^{q}(A)
$$

is an equality. 
Proof. See [44, Lemma 6.13].

We will use this lemma to see that the family constructed later by formal $q$ expansions comes from some $\Lambda$-adic form defined here (after Theorem 25).

\section{Klingen Eisenstein series}

Now we recall the notion of Klingen Eisenstein series in the totally real case. The $p$-adic constructions are just special cases of [50] (this is slightly more general than in [44] since we allow nearly ordinary forms instead of only ordinary forms). For the $\ell$-adic construction we just follow [44].

\subsection{Induced representations.}

4.1.1. Archimedean picture. Let $(\pi, H)$ be a unitary Hilbert representation of $\mathrm{GL}_{2}(\mathbb{R})$ and $H_{\infty}$ be the smooth vectors. Let $\chi$ be the central character of $\pi$, and let $\psi$ and $\tau$ be unitary characters of $\mathbb{C}^{\times}$such that $\left.\psi\right|_{\mathbb{R}^{x}}=\chi$. As in [44], we can use $\pi$ and $\psi$ to define a representation of $\operatorname{GU}(1,1)$ which we denote as $\pi_{\psi}$. Now we define a representation $\rho$ of $P(\mathbb{R})$ in $H$ : for $p=m n, n \in N_{P}(\mathbb{R}), m=m(b x$, $a) \in M_{P}(\mathbb{R})$ with $a, b \in \mathbb{C}^{\times}, x \in \mathrm{GL}_{2}(\mathbb{R})$, we define

$$
\rho(p) v:=\tau(a) \psi(b) \pi(x) v, \quad v \in H .
$$

We define a representation by smooth induction $I\left(H_{\infty}\right):=\operatorname{Ind}_{P(\mathbb{R})}^{\mathrm{GU}(2,2)(\mathbb{R})} \rho$, and denote $I(\rho)$ as the space of $K_{\infty}$-finite vectors in $I\left(H_{\infty}\right)$. We also define for each $z \in \mathbb{C}$ a function

$$
f_{z}(g):=\delta(m)^{3 / 2+z} \rho(m) f(k), \quad g=m k \in P(\mathbb{R}) K_{\infty},
$$

where $\delta$ is such that $\delta^{3}=\delta_{P}$ for $\delta_{P}$ the modulus character of $P$, and an action of $\mathrm{GU}(2,2)(\mathbb{R})$ on it by

$$
(\sigma(\rho, z)(g) f)(k):=f_{z}(k g) .
$$

Denote $\eta=\left({ }_{-1}^{1}\right)$. Let $\left(\pi^{\vee}, V\right)$ be $H$ but with the action given by $\pi^{\vee}(x)=$ $\pi\left(\eta^{-1} x \eta\right)$ for $x$ in $\mathrm{GL}_{2}(\mathbb{R})$. Denote $\rho^{\vee}, I\left(\rho^{\vee}\right), I^{\vee}\left(H_{\infty}\right)$, and $\left.\sigma\left(\rho^{\vee}, z\right), I\left(\rho^{\vee}\right)\right)$ the representations and spaces defined as above but with $\pi, \psi, \tau$ replaced by $\pi^{\vee} \otimes(\tau$ odet $), \psi \tau \tau^{c}, \bar{\tau}^{c}$. Also, recall that, for any $z \in \mathbb{C}, f \in I\left(H_{\infty}\right)$, and $k \in K_{\infty}$ we have defined the intertwining operator

$$
A(\rho, z, f)(k):=\int_{N_{P}(\mathbb{R})} f_{z}(w n k) d n .
$$


Then $A(\rho, z,-) \in \operatorname{Hom}_{\mathbb{C}}\left(I\left(H_{\infty}\right), I^{\vee}\left(H_{\infty}\right)\right)$ intertwines the actions of $\sigma(\rho, z)$ and $\sigma\left(\rho^{\vee},-z\right)$.

We let $K_{\infty}^{\prime}=\mathrm{O}(2) \subset \mathrm{GL}_{2}$ and $K_{\infty,+}^{\prime}=\mathrm{SO}_{2}$. Suppose that $\pi_{\infty}$ is the holomorphic discrete series representation associated to the weight $\kappa \geqslant 6$. Then it is well known that there is a unique (up to scalar) vector $v \in \pi$ such that $k \cdot v=\operatorname{det} \mu(k, i)^{-\kappa}$ (here $\mu$ means the second component of the automorphic factor $J$ instead of the similitude character) for any $k \in K_{\infty}^{\prime}$. Then, as in $[44,9.2 .1]$, by the Frobenius reciprocity law there is a unique (up to scalar) vector $\tilde{v} \in I(\rho)$ such that $k \cdot \tilde{v}=\operatorname{det} \mu(k, i)^{-\kappa} \tilde{v}$ for any $k \in K_{\infty}^{+}$. We fix $v$ and scale $\tilde{v}$ such that $\tilde{v}(1)=v$. In $\pi^{\vee}, \pi(w) v(w$ is defined in Section 3.1) has the action of $K_{\infty}^{+}$given by multiplying by $\operatorname{det} \mu(k, i)^{-\kappa}$. There is a unique vector $\tilde{v}^{\vee} \in I\left(\rho^{\vee}\right)$ such that the action of $K_{\infty}^{+}$is given by $\operatorname{det} \mu(k, i)^{-\kappa}$ and $\tilde{v}^{\vee}(w)=\pi(w) v$. Then by uniqueness there is a constant $c(\rho, z)$ such that $A(\rho, z, \tilde{v})=c(\rho, z) \tilde{v}^{\vee}$.

Definition 29. We define $F_{\kappa} \in I(\rho)$ to be the $\tilde{v}$ as above.

4.1.2. Non-Archimedean picture. Let $v$ be a prime of $F$ and $(\pi, V)$ an irreducible admissible representation of $\mathrm{GL}_{2}\left(F_{v}\right)$ which is unitary and tempered, with central character $\chi$. Let $\psi$ and $\tau$ be unitary characters of $\mathcal{K}_{v}^{\times}$such that $\left.\psi\right|_{F_{v}^{\times}}=\chi$. We extend $\pi$ to a representation $\rho$ of $P\left(F_{v}\right)$ on $V$ as follows. For $p=m n, n \in N_{P}\left(F_{v}\right), m=m(b x, a) \in M_{P}\left(F_{v}\right), a, b \in \mathcal{K}_{v}^{\times}, x \in \mathrm{GL}_{2}\left(F_{v}\right)$, put

$$
\rho(g) v:=\tau(a) \psi(b) \pi(s) v \quad v \in V .
$$

Let $I(\rho)$ be the representation defined by admissible induction: $I(\rho)=$ $\operatorname{Ind}_{P\left(F_{v}\right)}^{\mathrm{GU}(2,2)\left(F_{v}\right)} \rho$. As in the Archimedean case, for each $f \in I(\rho)$ and each $z \in \mathbb{C}$ we define a function $f_{z}$ on $\mathrm{GU}\left(F_{v}\right)$ by

$$
f_{z}(g):=\delta(m)^{3 / 2+z} \rho(m) f(k), \quad g=m k \in P\left(F_{v}\right) K_{v}
$$

and a representation $\sigma(\rho, z)$ of $\mathrm{GU}(2,2)\left(F_{v}\right)$ on $I(\rho)$ by

$$
(\sigma(\rho, z)(g) f)(k):=f_{z}(k g) .
$$

Let $\left(\pi^{\vee}, V\right)$ be $V$ but the action given by $\pi^{\vee}(g)=\pi\left(\eta^{-1} g \eta\right)$. This representation is also tempered and unitary. We denote by $\rho^{\vee}, I\left(\rho^{\vee}\right)$, and $\left(\sigma\left(\rho^{\vee}, z\right), I\left(\rho^{\vee}\right)\right)$ the representations and spaces defined by replacing $\pi, \psi$ and $\tau$ by $\pi^{\vee} \otimes(\tau \circ \operatorname{det}), \psi \tau \tau^{c}$, and $\bar{\tau}^{c}$, respectively.

Also we define for $f \in I(\rho), k \in K_{v}$, and $z \in \mathbb{C}$ the intertwining operator

$$
A(\rho, z, v)(k):=\int_{N_{P}\left(F_{v}\right)} f_{z}(w n k) d n .
$$


As a consequence of our hypotheses on $\pi$ this integral converges absolutely and uniformly for $z$ and $k$ in compact subsets of $\left\{z: \operatorname{Re}(z)>\frac{3}{2}\right\} \times K_{v}$. As in [44, 9.1.3], this has a meromorphic continuation (in the sense defined there) to $\mathbb{C}$, and the poles can only occur when $\operatorname{Re}(z)=0, \pm \frac{1}{2}$. In the case when everything is unramified we define a spherical vector $F_{w}^{s p h}$ for the unique vector invariant under $\mathrm{GU}\left(\mathcal{O}_{F}\right)$ and $F_{\rho, w}(\mathrm{id})=\varphi^{u r}$ for $\varphi^{u r} \in \pi_{w}$ the unramified vector.

4.1.3. $p$-adic picture. Now assume that $v \mid p$. We need to study the relations between the $\mathrm{GL}_{2}$ picture and the computations in [50] for $\mathrm{U}(1,1)$. Suppose that $\pi_{v} \simeq \pi\left(\mu_{1}, \mu_{2}\right)$ where $\operatorname{val}_{p}\left(\mu_{1}(p)\right)=-(\kappa-1) / 2$ and $\operatorname{val}_{p}\left(\mu_{2}(p)\right)=(\kappa-1) / 2$. Later we may write $\mu_{1, v}$ and $\mu_{2, v}$ to indicate the dependence on $v$. Let $\psi, \tau, I(\rho)$, $I\left(\rho^{\vee}\right)$, and $A(\rho, z, v)$ be as before. From now on we write $\xi=\psi / \tau$ and $\xi=\left(\xi_{1}\right.$, $\xi_{2}$ ) with respect to $\mathcal{K}_{v} \equiv F_{v} \times F_{v}$, and similarly for $\tau_{1}, \tau_{2}, \psi_{1}, \psi_{2}$. Note that our $\xi$ here is different from that in [50]. In fact the $\xi_{1}, \xi_{2}, \chi_{1}, \chi_{2}$ there are $\mu_{1} \bar{\xi}_{2}, \mu_{1} \bar{\xi}_{1}$, $\mu_{1} \psi_{2}^{-1}, \mu_{2} \psi_{2}^{-1}$. Note that $\psi_{1} \psi_{2}=\mu_{1} \mu_{2}$.

Generic case. The generic case mentioned in [50] corresponds to $\operatorname{cond}\left(\chi_{1}\right)>$ $\operatorname{cond}\left(\tau_{2}\right)>\operatorname{cond}\left(\chi_{2}\right)>\operatorname{cond}\left(\tau_{1}\right)$ (note that the $\tau$ in $\rho^{\vee}$ is $\left.\bar{\tau}^{c}\right)$. We assume that

$$
\operatorname{cond}\left(\mu_{2}\right)=\operatorname{cond}\left(\psi_{2}\right)>\operatorname{cond}\left(\tau_{2}\right)>\operatorname{cond}\left(\psi_{1}\right)>\operatorname{cond}\left(\tau_{1}\right)>\operatorname{cond}\left(\mu_{1}\right)>0 \operatorname{~}
$$

Then the datum is generic in the sense of [50]. We write $p^{n_{1, v}}, p^{n_{2, v}}, p^{n_{3, v}}, p^{n_{4, v}}$ for the first four terms above.

4.1.4. Global picture. Let $(\pi, V)$ be a cuspidal automorphic representation of $\mathrm{GL}_{2}\left(\mathbb{A}_{F}\right)$, and let $\tau, \psi: \mathbb{A}_{\mathcal{K}}^{\times} \rightarrow \mathbb{C}^{\times}$be Hecke characters such that $\left.\psi\right|_{\mathbb{A}_{F}^{\times}}=\chi_{\pi}$. We let $\tau=\otimes \tau_{w}$ and $\psi=\otimes \psi_{w}$ be their local decompositions, for $w$ running over places of $F$. We define $I(\rho)$ to be the restricted product $\otimes I\left(\rho_{w}\right)$ with respect to the $F_{\rho, w}$ at those $w$ at which $\tau_{w}, \psi_{w}, \pi_{w}$ are unramified.

For each $z \in \mathbb{C}$ and $f \in I(\rho)$ we define a function $f_{z}$ on $G\left(\mathbb{A}_{F}\right)$ as

$$
f_{z}(g):=\otimes f_{w, z}\left(g_{w}\right)
$$

where $f_{w, z}$ are defined as before. Also we define an action $\sigma(\rho, z)$ of $\left(\mathfrak{g u}, K_{\infty}\right)$ $\otimes G\left(\mathbb{A}_{f}\right)$ on $I(\rho)$ by $\sigma(\rho, z):=\otimes \sigma\left(\rho_{w}, z\right)$. Similarly we define $\rho^{\vee}, I\left(\rho^{\vee}\right)$, and $\sigma\left(\rho^{\vee}, z\right)$ but with $\rho$ replaced by $\rho^{\vee}$.

4.1.5. Klingen-type Eisenstein series on $G$. Let $\pi, \psi$, and $\tau$ be as above. For $f \in I(\rho), z \in \mathbb{C}$, and $g \in \operatorname{GU}(2,2)\left(\mathbb{A}_{F}\right)$, the series

$$
E(f, z, g):=\sum_{\gamma \in P(F) \backslash G(F)} f_{z}(\gamma g)
$$


is known to converge absolutely and uniformly for $(z, g)$ in compact subsets of

$$
\left\{z \in \mathbb{C}: \operatorname{Re}(z)>\frac{3}{2}\right\} \times \operatorname{GU}(2,2)\left(\mathbb{A}_{F}\right) .
$$

We call this series the Klingen Eisenstein series (see [35, II 1.5]).

\subsection{Explicit local sections.}

4.2.1. Archimedean sections. The Klingen subsection at each place dividing $\infty$ is the $F_{\kappa}$ defined before.

4.2.2. Prime to $p$ sections. Let $v \in \Sigma$ be a prime of $F$ not dividing $p$. Let $\left(\varpi_{v}^{r_{\psi}}\right.$ ) and $\left(\varpi_{v}^{s}\right)$ be the conductors of $\psi_{v}$ and $\xi_{v}$. The sections chosen here are the same as in [44, Section 9], which we briefly recall. For $K \subseteq K_{r, s}$ with $r \geqslant \max \left(r_{\psi}, s\right)$ we define a character $v$ of $K_{r, s}$ by

$$
v\left(\left(\begin{array}{cc}
a & b \\
c & d \\
* & *
\end{array}\right)\right):=\psi(a d-b c) \bar{\xi}(d) .
$$

Let $\varphi \in V$ be any vector having a conductor with respect to $\pi^{\vee}$, and let $\left(\lambda^{r_{\varphi}}\right):=$ $\operatorname{cond}_{\pi^{\vee}}(\varphi)$. For any $K_{r, t}$ with $r \geqslant \max \left(r_{\psi}, r_{\varphi}, s\right)$ and $t \geqslant s$ we define $F_{\varphi, r, t} \in I(\rho)$ by

$$
F_{\varphi, r, t}(g):= \begin{cases}v(k) \rho(p) \varphi & g=p m k \in P\left(\mathcal{O}_{F, v}\right) w K_{r, t} \\ 0 & \text { otherwise. }\end{cases}
$$

4.2.3. p-adic sections. Let $K_{v, 0}$ consist of matrices $\left(\begin{array}{llll}k_{11} & k_{12} & k_{13} & k_{14} \\ k_{21} & k_{22} & 23 \\ k_{31} & k_{32} & k_{24} \\ k_{41} & k_{42} & k_{43} & k_{34}\end{array}\right)$ in $\mathrm{GL}_{4}\left(\mathcal{O}_{F, v}\right)$ such that the under diagonal entries of the first column are divisible by $p^{n_{1, v}}$, the under diagonal entries of the second column are divisible by $p^{n_{2, v}}$, and $k_{34}$ is divisible by $p^{n_{3, v}}$. Let $K_{v, 1} \subset K_{v}$ be the set of matrices such that $k_{11} \equiv 1 \bmod p^{n_{1, v}}$, $k_{22} \equiv 1 \bmod p^{n_{2, v}}, k_{33} \equiv \bmod p^{n_{3, v}}, k_{44} \equiv 1 \bmod p^{n_{4, v}}$. We define our $p$-adic subsection $F_{v}^{0}$ to be the one supported in $P\left(\mathbb{Q}_{p}\right) K_{v, 1}$ which takes value $p^{-n_{1, v}-n_{3, v}}$ on the identity and is invariant under $K_{v, 1}$. This is nearly ordinary as proved in [50] (see also [44, Proposition 9.15]).

\subsection{Good Eisenstein series.}

4.3.1. Eisenstein datum. Let $(\pi, V)$ be an irreducible cuspidal unitary automorphic representation of $\mathrm{GL}_{2}\left(\mathbb{A}_{F}\right)$ with central character $\chi$, and let $V=\otimes V_{w}$ and $\pi=\otimes \pi_{w}$. Suppose that the Galois character corresponding to $\chi$ factors through $\Gamma_{\mathcal{K}}$. By an Eisenstein datum for $\pi$ we will mean a 4-tuple 
$\mathcal{D}=\{\Sigma, \varphi, \psi, \tau\}$ consisting of a finite set of primes $\Sigma$, a cusp form $\varphi \in V$ that is completely reducible $\varphi=\otimes \varphi_{v}$, and unitary Hecke characters $\psi=\otimes \psi_{w}$ and $\tau=\otimes \tau_{w}$ of $\mathbb{A}_{\mathcal{K}}^{\times} / \mathcal{K}^{\times}$satisfying the following.

- $\Sigma$ contains all primes dividing $p$, primes ramified in $\mathcal{K} / \mathbb{Q}$, and all primes $v$ such that $\pi_{v}, \psi_{v}$ or $\tau_{v}$ is ramified.

- For all $k \in K_{\infty}^{\prime}, \pi_{\infty}(k) \varphi_{\infty}=j(k, i)^{-\kappa} \varphi_{\infty}$.

- If $v \notin \Sigma$, then $\varphi_{v}$ is the newvector.

- If $v \in \Sigma, v \nmid p$, then $\varphi_{v}$ has a conductor with respect to $\pi_{v}^{\vee}$.

- If $v \mid p$, then $\varphi_{v}$ is the nearly ordinary vector.

- $\left.\psi\right|_{\mathbb{A}_{F}^{\times}}=\chi$.

- $\tau_{v}(x)=(x /|x|)^{-\kappa}=\psi_{v}(x)$ for any $x \in F_{v}$ and $v \mid \infty$.

We remark that all the above are similar to $[44,9.3 .1]$ except that for $v \mid p$ we are allowing nearly ordinary (not just ordinary) vectors. Also we assume that the $\chi_{0}$ in [44] is trivial for simplicity.

Let $\xi=\otimes \xi_{w}=\psi / \tau$, and define $F:=\otimes_{v \mid \infty} F_{\kappa} \prod_{v \notin \Sigma} F_{\rho, v} \otimes_{v \in \Sigma, v \nmid p} F_{\varphi, r, t} \otimes_{v \mid p} F_{v}^{0}$. We define $E_{\mathcal{D}}(z, g)=E(F, z, g)$ to be the Klingen Eisenstein series associated to the subsection $F$. For any parabolic subgroup $R$ of $G$ and an automorphic form $\varphi$ on $G$ we define the constant term of $\varphi$ along $R$ to be

$$
\varphi_{R}:=\int_{N_{R}(F) \backslash N_{R}\left(\mathbb{A}_{F}\right)} \varphi_{R}(n g) d n .
$$

Then we have the following straightforward generalization of [44, Lemma 9.7]. The proofs are completely the same.

Lemma 30. Suppose that $\kappa>6$, and let $z_{\kappa}:=(\kappa-3) / 2$. Let $F=F_{\kappa} \otimes F_{f} \in$ $I(\rho)=I\left(\rho_{\infty}\right) \otimes I\left(\rho_{f}\right)$.

(1) $A\left(\rho, z_{\kappa}, F\right)=0$.

(2) $E\left(F, z_{\kappa}, g\right)_{P}=F_{z_{\kappa}}(g)$.

Let $\kappa>6$. Then for any $F=F_{\kappa} \otimes F_{f} \in I(\rho)$ we define a function of $(Z, x) \in$ $\mathbf{H}_{2}^{\Sigma_{\infty}} \otimes G\left(\mathbb{A}_{F, f}\right)$ :

$$
E(Z, x ; F):=J(g, i)^{\kappa} \mu(g)^{\kappa} E\left(F, z_{\kappa}, g x\right), \quad g \in G U(2,2)^{+}\left(F_{\infty}\right), g(i)=Z .
$$

Here $J(g, i)=\operatorname{det}\left(C_{g} i+D_{g}\right)$ for $g=\left(\begin{array}{cc}A_{g} & B_{g} \\ C_{g} & D_{g}\end{array}\right)$. The following proposition is essentially [44, Proposition 9.8]. 
Proposition 31. Suppose that $\kappa>6$ and $F=F_{\kappa} \otimes F_{f}$. Then $E(Z, x ; F)$ is a holomorphic modular form of weight $\kappa$.

Definition 32. We write $E\left(F, z_{\kappa}, g\right)=\sum_{\beta \in S_{2}(F)} \mu_{\mathcal{D}}(\beta, F, g)$ for the Fourier expansion of $E\left(F, z_{\kappa}, g\right)$ at $g$.

4.4. Hecke operators. We will recall the definitions for the Hecke operators at the unramified primes at the beginning of Section 9, and some Hecke polynomials $Q_{w}$ for places $w$ of $\mathcal{K}$, which are essentially those given in [44, 9.5]. We let $\mathbf{h}=\mathbf{h}^{\Sigma}$ be the abstract Hecke algebra generated by Hecke operators introduced there at primes outside $\Sigma$. Define $\lambda_{\mathcal{D}}: \mathbf{h}^{\Sigma} \rightarrow \mathbb{C}$ by $h \cdot E_{\mathcal{D}}=\lambda_{\mathcal{D}}(h) E_{\mathcal{D}}$. We record the following generalization of proposition [44, Proposition 9.14].

Proposition 33. Suppose that $\kappa>6$ and $w \notin \Sigma$. Then $\lambda_{\mathcal{D}}\left(Q_{w}\right)\left(q_{v}^{-s}\right)$ is given by the Euler factor at $w$ :

$$
L_{\mathcal{K}}^{\Sigma}\left(f, \bar{\xi}^{c} \psi^{c}, s-2\right) L^{\Sigma}\left(\psi^{c}, s-3\right) L^{\Sigma}\left(\chi \bar{\xi}^{\prime} \psi^{c}, s-\kappa\right)
$$

where $L_{\mathcal{K}}^{\Sigma}\left(f, \bar{\xi}^{c} \psi^{c}, s-2\right)$ is the corresponding L-function for $f$ twisted by the character $\bar{\xi}^{c} \psi^{c}$ over $\mathcal{K}$, with the Euler factors at primes dividing $\Sigma$ removed.

The proof is completely the same as that in [44] (there is no difference between the local situations for $F=\mathbb{Q}$ and general $F$ ).

\section{Hermitian theta functions}

We generalize results in $[44$, Section 10$]$ in this section.

\subsection{Weil Representations.}

The local set-up. Let $v$ be a place of $F$. Let $h \in S_{2}\left(F_{v}\right)$, det $h \neq 0$. Let $\mathrm{U}_{h}$ be the unitary group of this matrix, and denote $V_{v}$ as the corresponding Hermitian space. Let $V_{1}:=\mathcal{K}_{v} \oplus \mathcal{K}_{v}:=X_{v} \oplus Y_{v}$ be the Hermitian space associated to $\mathrm{U}(1,1)$. Let $W:=V_{v} \otimes_{\mathcal{K}_{v}} V_{1, v}$. Then

$$
(-,-):=\operatorname{Tr}_{\mathcal{K}_{v} / F_{v}}\left(\langle-,-\rangle_{h} \otimes_{\mathcal{K}_{v}}\langle-,-\rangle_{1}\right)
$$

is an $F_{v}$ linear pairing on $W$ that makes $W$ into an eight-dimensional symplectic space over $F_{v}$. The canonical embedding of $\mathrm{U}_{h} \times \mathrm{U}_{1}$ into $\operatorname{Sp}(W)$ realizes the pair $\left(\mathrm{U}_{h}, \mathrm{U}_{1}\right)$ as a dual pair in $\operatorname{Sp}(W)$. Let $\lambda_{v}$ be a character of $\mathcal{K}_{v}^{\times}$such that $\left.\lambda_{v}\right|_{F_{v}^{\times}}=$ $\chi_{\mathcal{K} / F, v}^{2}$. It is well known (see [28]) that there is a splitting $\mathrm{U}_{h}\left(F_{v}\right) \times \mathrm{U}_{1}\left(F_{v}\right) \hookrightarrow$ $\operatorname{Mp}\left(W, F_{v}\right)$ of the metaplectic cover $\operatorname{Mp}\left(W, F_{v}\right) \rightarrow \operatorname{Sp}\left(W, F_{v}\right)$ determined by the 
character $\lambda_{v}$. This gives the Weil representation $\omega_{h, v}(u, g)$ of $\mathrm{U}_{h}\left(F_{v}\right) \times \mathrm{U}_{1}\left(F_{v}\right)$ where $u \in \mathrm{U}_{h}\left(F_{v}\right)$ and $g \in \mathrm{U}_{1}\left(F_{v}\right)$, via the Weil representation of $\operatorname{Mp}\left(W, F_{v}\right)$ on the space of Schwartz functions $\mathcal{S}\left(V_{v} \otimes_{\mathcal{K}_{v}} X_{v}\right)$. Moreover we write $\omega_{h, v}(g)$ to mean $\omega_{h, v}(1, g)$. For $X \in M_{2 \times 1}\left(\mathcal{K}_{v}\right)$, we define $\langle X, X\rangle_{h}:={ }^{t} \bar{X} \beta X$. We record here some useful formulas for $\omega_{h, v}$ which are generalizations of the formulas in [44, Section 10].

- $\omega_{h, v}(u, g) \Phi(X)=\omega_{h, v}(1, g) \Phi\left(u^{-1} X\right)$.

- $\omega_{h, v}\left(\operatorname{diag}\left(A,{ }^{t} \bar{A}^{-1}\right)\right) \Phi(X)=\lambda(\operatorname{det} A)|\operatorname{det} A|_{\mathcal{K}} \Phi(X A)$.

- $\omega_{h, v}(r(S)) \Phi(x)=\Phi(x) e_{v}\left(\operatorname{tr}\langle X, X\rangle_{h} S\right)$.

- $\omega_{h, v}(\eta) \Phi(x)=|\operatorname{det} h|_{v} \int \Phi(Y) e_{v}\left(\operatorname{Tr}_{\mathcal{K}_{v} / F_{v}}\left(\operatorname{tr}\langle Y, X\rangle_{h}\right)\right) d Y$.

Global set-up. Let $h \in S_{2}(F), h>0$. We can define global versions of $\mathrm{U}_{h}, \mathrm{GU}_{h}$, $W$, and $(-,-)$, analogous to the local case. Fixing an idele class character $\lambda=$ $\otimes \lambda_{v}$ of $\mathbb{A}_{\mathcal{K}}^{\times} / \mathcal{K}^{\times}$such that $\left.\lambda\right|_{F^{\times}}=\chi_{\mathcal{K} / F}^{2}$, the associated local splitting described above then determines a global splitting

$$
\mathrm{U}_{h}\left(\mathbb{A}_{F}\right) \times \mathrm{U}_{1}\left(\mathbb{A}_{F}\right) \hookrightarrow \operatorname{Mp}\left(W, \mathbb{A}_{F}\right)
$$

and hence an action $\omega_{h}:=\otimes \omega_{h, v}$ of $\mathrm{U}_{h}\left(\mathbb{A}_{F}\right) \times \mathrm{U}_{1}\left(\mathbb{A}_{F}\right)$ on the Schwartz space $\mathcal{S}\left(V_{\mathbb{A}_{\mathcal{K}}} \otimes X\right)$.

Theta functions. Given $\Phi \in \mathcal{S}\left(V_{\mathbb{A}_{F}}\right)$, we let

$$
\Theta_{h}(u, g ; \Phi):=\sum_{x \in V} \omega_{h}(u, g) \Phi(x) .
$$

This is an automorphic form on $\mathrm{U}_{h}\left(\mathbb{A}_{F}\right) \times \mathrm{U}_{1}\left(\mathbb{A}_{F}\right)$.

5.2. Some useful Schwartz functions. We now record some Schwartz functions that will show up later on and their properties. These are straightforward generalizations of [44, Section 10] with some modifications. We have been keeping the presentation parallel to that in [44] for convenience.

5.2.1. Archimedean Schwartz functions. Suppose that $v \mid \infty$. Let $\Phi_{h, v} \in \mathcal{S}(V \otimes \mathbb{R})$ be

$$
\Phi_{h, v}(x)=e^{-2 \pi\langle x, x\rangle_{h}} .
$$

Let

$$
\lambda_{v}(z)=(z /|z|)^{-2} .
$$

Recall that $\mathfrak{h}$ is the Poincare half plane. 
Lemma 34. Given $z \in \mathfrak{h}$, let $\Phi_{h, z}(x):=e\left(\langle x, x\rangle_{h} z\right)\left(\right.$ so $\left.\Phi_{h, i}=\Phi_{h, \infty}\right)$. For any $g \in U_{1}(\mathbb{R})$,

$$
\omega_{h}(g) \Phi_{h, z}=J_{1}(g, z)^{-2} \Phi_{h, g(z)} .
$$

In particular, if $k \in K_{\infty, 1}^{+}$then $\omega_{h}(k) \Phi_{h, \infty}=J_{1}(k, i)^{-2} \Phi_{h, \infty}$.

Proof. The proof is just [44, Lemma 10.1].

5.2.2. Schwartz functions at finite places. For a finite place $v$ of $F$ dividing a rational prime $\ell$, let $\Phi_{0} \in \mathcal{S}\left(V_{v}\right)$ be the characteristic function of the set of column vectors with entries in $\mathcal{O}_{\mathcal{K}, v}$. For $y \in \mathrm{GL}_{2}\left(\mathcal{K}_{v}\right)$ we let $\Phi_{0, y}(x):=\Phi_{0}\left(y^{-1} x\right)$.

Lemma 35. Let $h \in S_{2}\left(F_{v}\right)$, det $h \neq 0$. Let $y \in \mathrm{GL}_{2}\left(\mathcal{K}_{v}\right)$. Suppose that ${ }^{t} \bar{y} h y \in$ $S_{2}\left(\mathcal{O}_{F, v}\right)^{\times}$. (In this paper we use $S_{2}\left(\mathcal{O}_{F, v}\right)^{*}$ to mean to dual of $S_{2}\left(\mathcal{O}_{F, v}\right)$ under the paring on $S_{2}\left(F_{v}\right)$ defined by $(x, y) \mapsto \operatorname{tr}(x y)$.)

(i) If $\lambda$ is unramified, $v$ is unramified in $\mathcal{K}$, and $h, y \in \mathrm{GL}_{2}\left(\mathcal{O}_{F, v}\right)$, then

$$
\omega_{h}\left(U_{1}\left(\mathcal{O}_{F, v}\right)\right) \Phi_{0, y}=\Phi_{0, y} .
$$

(ii) If $D_{v} \operatorname{det}^{t} \bar{y} h y \mid \varpi_{v}^{r}, r>0$, then

$$
\omega_{h}(k) \Phi_{0, y}=\lambda\left(a_{k}\right) \Phi_{0, y}, \quad k \in \mathrm{U}_{1}\left(\mathcal{O}_{F, v}\right), \varpi_{v}^{r} \mid c_{k} .
$$

Proof. See [44, Lemma 10.2].

Let $\theta$ be a character of $\mathcal{K}_{v}^{\times}$, and let $0 \neq x \in \operatorname{cond}(\theta)$. Let

$$
\Phi_{\theta, x}(u):=\sum_{a \in\left(\mathcal{O}_{\mathcal{K}, v} / x\right)^{\times}} \theta(a) \Phi_{0}\left({ }^{t}\left(u_{1}+a / x, u_{2}\right)\right), \quad u={ }^{t}\left(u_{1}, u_{2}\right) .
$$

For $y \in \mathrm{GL}_{2}\left(\mathcal{K}_{v}\right)$ we let $\Phi_{\theta, x, y}(u):=\Phi_{\theta, x}\left(y^{-1} u\right)$. We let $\Phi_{h, \theta, x}:=\omega_{h}\left(\eta^{-1}\right) \Phi_{\theta, x}$ and $\Phi_{h, \theta, x, y}:=\omega_{h}\left(\eta^{-1}\right) \Phi_{\theta, x, y}$.

Lemma 36. Let $h \in S_{2}\left(F_{v}\right)$, det $h \neq 0$. Let $y \in \mathrm{GL}_{2}\left(\mathcal{K}_{v}\right)$. Suppose that $\bar{y}^{t} h y \in$ $S_{2}\left(\mathcal{O}_{F, v}\right)^{*}$. Let $\theta$ be a character of $\mathcal{K}_{v}^{\times}$, and let $0 \neq x \in \operatorname{cond}(\theta)$ be such that $\varpi_{v} \mid x$. Let $(c):=\operatorname{cond}(\theta) \cap\left(\tilde{\varpi}_{v}\right)$ where $\tilde{\varpi}_{v}=\varpi_{v}$ if $v$ splits in $\mathcal{K}\left(\right.$ that is, $\tilde{\varpi}_{v}=\left(\varpi_{v}, \varpi_{v}\right)$ for $\varpi_{v}$ the uniformizer of $\left.F_{v}\right)$, and $\tilde{\varpi}_{v}$ is a uniformizer of $\mathcal{K}_{v}$ at $v$ is non-split in $\mathcal{K}$.

(i) If $c D_{v} \operatorname{det}^{t} \bar{y} h y \| x$ and $y^{-1} h y \in \mathrm{GL}_{2}\left(\mathcal{O}_{F, v}\right)$, and $D_{v}=1$ or $y^{-1} h^{-1 t} y^{-1}=$ $\left(\begin{array}{l}* * * \\ * d\end{array}\right)$ with $d \in \mathcal{O}_{F, v}$, then

$$
\omega_{h}(k) \Phi_{\theta, x, y}=\lambda \theta\left(a_{k}\right) \Phi_{\theta, x, y}, \quad k \in \mathrm{U}_{1}\left(\mathcal{O}_{F, v}\right), \mathfrak{d}^{-1} D_{v}\left|c_{k}, \mathfrak{d}^{-1} x \bar{x}\right| b_{k} .
$$


(ii) If $h=\operatorname{diag}(\alpha, \beta)$, then $\Phi_{h, \theta, x, y}$ is supported on the lattice $h^{-1 t} y^{-1} L_{\theta, x}^{*}$ where if $v$ is nonsplit in $\mathcal{K}$ then

$$
L_{\theta, x}^{*}=\left\{\left(u_{1}, u_{2}\right)^{t}: u_{2} \in \delta_{\mathcal{K}}{ }^{-1} \mathcal{O}_{\mathcal{K}, v}, \bar{u}_{1} \in \frac{x}{c \delta_{\mathcal{K}}}\left\{\begin{array}{l}
\mathcal{O}_{\mathcal{K}, v}, \operatorname{cond}(\theta)=\mathcal{O}_{\mathcal{K}, v}, \\
\mathcal{O}_{\mathcal{K}, v}^{\times}, \operatorname{cond}(\theta) \neq \mathcal{O}_{\mathcal{K}, v},
\end{array}\right\}\right.
$$

and if $v$ splits in $\mathcal{K}$, then

$$
L_{\theta, x}^{*}:=\left\{\left(u_{1}, u_{2}\right)^{t}: u_{2} \in \delta_{\mathcal{K}}^{-1} \mathcal{O}_{\mathcal{K}, v}, \bar{u}_{1, i} \in \frac{x_{i}}{c_{i} \delta_{\mathcal{K}}}\left\{\begin{array}{l}
\mathcal{O}_{F, v}, \operatorname{cond}(\theta)=\mathcal{O}_{F, v}, \\
\mathcal{O}_{F, v}^{\times}, \operatorname{cond}(\theta) \neq \mathcal{O}_{F, v},
\end{array}\right\}\right.
$$

with $\bar{u}_{1}=\left(\bar{u}_{1,1}, \bar{u}_{1,2}\right), x=\left(x_{1}, x_{2}\right), c=\left(c_{1}, c_{2}\right) \in \mathcal{K}_{v}=F_{v} \times F_{v}$, and $\theta=\left(\theta_{1}\right.$, $\left.\theta_{2}\right)$. Furthermore, for $v=h^{-1 t} y^{-1} u$ with $u \in L_{\theta, x}^{*}$,

$$
\Phi_{h, \theta, x, y}(v)=|\operatorname{det} h y \bar{y}|_{v} D_{v}^{-1} \lambda(-1) \sum_{a \in\left(\mathcal{O}_{\mathcal{K}, v} / x\right)^{\times}} \theta(s) e_{\ell}\left(\operatorname{Tr}_{\mathcal{K} / \mathbb{Q}} a \bar{u}_{1} / x\right)
$$

Proof. See [44, Lemma 10.3].

Lemma 37. Suppose that $v \mid p$ splits in $\mathcal{K}$. Let $(c):=\operatorname{cond}(\theta)$ and suppose that $c=\left(p^{r}, p^{s}\right)$ with $r, s>0$. Let $\gamma=(\eta, 1) \in \mathrm{SL}_{2}\left(\mathcal{O}_{\mathcal{K}, v}\right)=\operatorname{SL}_{2}\left(\mathcal{O}_{F, v}\right) \times \operatorname{SL}_{2}\left(\mathcal{O}_{F, v}\right)$. Suppose that $h=\operatorname{diag}(\alpha, \beta)$ with $\alpha, \beta \in F_{v}^{\times}$. Then the following hold.

(i) $\Phi_{h, \theta, c, \gamma}$ is supported on

$$
L^{\prime}:=\left\{u=(a, b)^{t}: a \in \mathcal{O}_{F, v}^{\times} \times \mathcal{O}_{F, v}, b \in \mathcal{O}_{F, v} \times \mathcal{O}_{F, v}^{\times}\right\}
$$

and for $u \in L^{\prime}$

$$
\Phi_{h, \theta, c, \gamma}(u)=\theta_{1}^{-1}\left(\alpha a_{2}\right) \mathfrak{g}\left(\theta_{1}\right) \theta_{2}^{-1}\left(\beta b_{1}\right) \mathfrak{g}\left(\theta_{2}\right)
$$

where $a=\left(a_{1}, a_{2}\right), b=\left(b_{1}, b_{2}\right) \in \mathcal{O}_{F, v} \times \mathcal{O}_{F, v}$, and $\theta=\left(\theta_{1}, \theta_{2}\right)$.

(ii) $\omega_{h}(u, k) \Phi_{h, \theta, c}=\theta_{1}^{-1}\left(a_{g}\right) \theta_{2}\left(d_{g}\right) \lambda \theta\left(d_{k}\right) \Phi_{h, \theta, c}$ for $u=\left(g, g^{\prime}\right) \in \mathrm{U}_{h}\left(\mathbb{Z}_{p}\right)$ with $p^{\max (r, s)} \mid c_{g}$ and for $k \in \mathrm{U}_{1}\left(\mathbb{Z}_{p}\right)$ such that $p^{\max (r, s)} \mid c_{k}$. Here we write $k=\left(\begin{array}{ll}a_{k} & b_{k} \\ c_{k} & d_{k}\end{array}\right)$ and $g=\left(\begin{array}{ll}a_{g} & b_{g} \\ c_{g} & d_{g}\end{array}\right)$.

Proof. See [44, Lemma 10.4]. 


\section{Siegel Eisenstein series and their pullbacks}

For the reader's convenience we try to present the work in a parallel way to that in [44, Section 11]. We remark that the differences in the formulas from those in [44] are mostly due to the discriminant and the unit group of $F$ and that we are allowing nearly ordinary forms (not only ordinary forms). Also in [44] they used symbols $\chi$ and $\tau$ for the same object. We make the corresponding notation consistent here.

From now on we always write $\xi=\psi / \tau$ once $\psi$ and $\tau$ are defined.

6.1. Some isomorphisms and embeddings. We recall the notation of $[44$, 11.1]. Let $V_{n}:=\mathcal{K}^{2 n}$. Then $w_{n}$ defines a skew-Hermitian pairing $\langle-,-\rangle_{n}$ on $V_{n}:\langle x, y\rangle_{n}:=x w_{n}{ }^{t} \bar{y}$. The group $G_{n} / F$ is the unitary similitude group $\mathrm{GU}\left(V_{n}\right)$ of the Hermitian space $\left(V_{n},\langle-,-\rangle_{n}\right)$. Let $W_{n}:=V_{n+1} \oplus V_{n}$ and $W_{n}^{\prime}: V_{n} \oplus V_{n}$. The matrices $w_{n+1} \oplus-w_{n}$ and $w_{n} \oplus-w_{n}$ define Hermitian pairings on $W_{n}$ and $W_{n}^{\prime}$, respectively.

One can define isomorphisms $\alpha_{n}: \mathrm{GU}\left(W_{n}\right) \simeq G_{2 n+1}, \alpha_{n}^{\prime}: \mathrm{GU}\left(W_{n}^{\prime}\right) \simeq G_{2 n}$, $\gamma_{n}: \mathrm{GU}\left(W_{n}\right) \simeq G_{2 n+1}$, and $\gamma_{n}^{\prime}: \mathrm{GU}\left(W_{n}^{\prime}\right) \simeq G_{2 n}$. We omit the details and refer to [44, 11.2.1]. Also as in [44] we use $S$ and $S^{\prime}$ to denote the matrices

$$
\left(\begin{array}{cccccc}
1 & & & & & \\
& 1 & & & & \\
& & 1 & & & \\
& & -1 & 1 & & \\
& & & & 1 & \\
-1 & & & & & 1
\end{array}\right)
$$

and

$$
\left(\begin{array}{ccccc}
1 & & & \\
& & 1 & & \\
& -1 & 1 & \\
-1 & & & & 1
\end{array}\right) \text {. }
$$

6.2. Siegel Eisenstein series on $\boldsymbol{G}_{n}$. Let $Q=Q_{n}$ be the Siegel parabolic subgroup of $\mathrm{GU}_{n}$ consisting of matrices $\left(\begin{array}{cc}A_{q} & B_{q} \\ 0 & D_{q}\end{array}\right)$. For a place $v$ of $F$ and a character $\tau$ of $\mathcal{K}_{v}^{\times}$we let $I_{n}(\tau)$ be the space of smooth $K_{n, v}$-finite functions $f$ : $K_{n, v} \rightarrow \mathbb{C}$ such that $f(q k)=\tau\left(\operatorname{det} D_{q}\right) f(k)$ for all $q \in Q_{n}\left(F_{v}\right) \cap K_{n, v}$ (we write $q$ as block matrix $q=\left(\begin{array}{cc}A_{q} & B_{q} \\ 0 & D_{q}\end{array}\right)$ ). Given $z \in \mathbb{C}$ and $f \in I(\tau)$ we define a function 
$f(z,-): G_{n}\left(F_{v}\right) \rightarrow \mathbb{C}$ by $\left.f(z, q k):=\tau\left(\operatorname{det} D_{q}\right)\right)\left|\operatorname{det} A_{q} D_{q}^{-1}\right|_{v}^{z+n / 2} f(k)$, $q \in Q_{n}\left(F_{v}\right)$ and $k \in K_{n, v}$.

For an idele class character $\tau=\otimes \tau_{v}$ of $\mathbb{A}_{\mathcal{K}}^{\times}$we similarly define a space $I_{n}(\tau)$ of smooth functions on $\prod_{v} K_{n, v}$. We also similarly define $f(z,-)$ given $f \in I_{n}(\tau)$ and $z \in \mathbb{C}$. There is an identification $\otimes I_{n}\left(\tau_{v}\right)=I_{n}(\tau)$, the former being the restricted tensor product defined using the spherical vectors $f_{v}^{s p h} \in I_{n}\left(\tau_{v}\right)$, $f_{v}^{s p h}\left(K_{n, v}\right)=1$, at the finite places $v$ where $\chi_{v}$ is unramified: $\otimes f_{v}$ is identified with $k \mapsto \prod_{v} f_{v}\left(k_{v}\right)$. Let $\mathcal{U} \subseteq \mathbb{C}$ be an open set. By a meromorphic section of $I_{n}(\tau)$ on $\mathcal{U}$ we mean a function $\varphi: \mathcal{U} \mapsto I_{n}(\tau)$ taking values in a finite-dimensional subspace $V \subset I_{n}(\tau)$ and such that $\varphi: \mathcal{U} \rightarrow V$ is meromorphic.

Let $\tau=\otimes \tau_{v}$ be a unitary idele class character of $\mathbb{A}_{\mathcal{K}}^{\times}$. For $f \in I_{n}(\tau)$ we consider the Eisenstein series

$$
E(f ; z, g):=\sum_{\gamma \in Q_{n}(F) \backslash G_{n}(F)} f(z, \gamma g) .
$$

This series converges absolutely and uniformly for $(z, g)$ in compact subsets of $\{\operatorname{Re}(z)>n / 2\} \times G_{n}\left(\mathbb{A}_{F}\right)$ and defines an automorphic form on $G_{n}$ and a holomorphic function on $\{\operatorname{Re}(z)>n / 2\}$. The Eisenstein series $E(f ; z, g)$ has a meromorphic continuation in $z$ to all of $\mathbb{C}$. If $\varphi: \mathcal{U} \rightarrow I_{n}(\tau)$ is a meromorphic section, then we put $E(\varphi ; z, g)=E(\varphi(z) ; z, g)$. This is clearly a meromorphic function of $z \in \mathcal{U}$ and an automorphic form on $G_{n}$ for those $z$ where it is holomorphic.

6.3. Pullbacks of Siegel Eisenstein series. Now we follow [44, 11.2] closely to recall the pullback formulas. However we repeat it here since the conventions are slightly different.

6.3.1. The pullback formulas. Let $\tau$ be a unitary idele class character of $\mathbb{A}_{\mathcal{K}}^{\times}$. Given a cusp form $\varphi$ on $G_{n}$ we consider

$$
\begin{aligned}
& F_{\varphi}(f ; z, g):=\int_{U_{n}\left(\mathbb{A}_{F}\right)} f\left(z, \gamma\left(g, g_{1} h\right)\right) \bar{\tau}\left(\operatorname{det} g_{1} g\right) \varphi\left(g_{1} h\right) d g_{1}, \\
& f \in I_{m+n}(\tau), g \in G_{m}\left(\mathbb{A}_{F}\right), h \in G_{n}\left(\mathbb{A}_{F}\right), \mu_{m}(g)=\mu_{n}(h), m=n+1 \text { or } n,
\end{aligned}
$$

with $\gamma=\gamma_{n}$ or $\gamma_{n}^{\prime}$ depending on whether $m=n+1$ or $m=n$. This is independent of $h$. The pullback formulas are the identities in the following proposition.

PROPOSITION 38. Let $\tau$ be a unitary idele class character of $\mathbb{A}_{\mathcal{K}}^{\times}$.

(i) If $f \in I_{2 n}(\tau)$, then $F_{\varphi}(f ; z, g)$ converges absolutely and uniformly for $(z, g)$ in compact sets of $\{\operatorname{Re}(z)>n\} \times G_{n}\left(\mathbb{A}_{F}\right)$, and for any $h \in G_{n}\left(\mathbb{A}_{F}\right)$ 
such that $\mu_{n}(h)=\mu(g)$

$$
\int_{\mathrm{U}_{n}(F) \backslash \mathrm{U}_{n}\left(\mathbb{A}_{F}\right)} E\left(f ; z, \gamma_{n}^{\prime}\left(g, g_{1} h\right)\right) \bar{\tau}\left(\operatorname{det} g_{1} h\right) \varphi\left(g_{1} h\right) d g_{1}=F_{\varphi}(f ; z, g) \text {. }
$$

(ii) If $f \in I_{2 n+1}(\tau)$, then $F_{\varphi}(f ; z, g)$ converges absolutely and uniformly for $(z, g)$ in compact sets of $\{\operatorname{Re}(z)>n+1 / 2\} \times G_{n+1}\left(\mathbb{A}_{F}\right)$ such that $\mu_{n}(h)=\mu_{n+1}(g)$

$$
\begin{gathered}
\int_{\mathrm{U}_{n}(F) \backslash \mathrm{U}_{n}\left(\mathbb{A}_{F}\right)} E\left(f ; z, \gamma_{n}\left(g, g^{\prime} h\right)\right) \bar{\tau}\left(\operatorname{det} g_{1} h\right) \varphi\left(g_{1} h\right) d g_{1} \\
=\sum_{\gamma \in P_{n+1}(F) \backslash G_{n+1}(F)} F_{\varphi}(f ; z, \gamma g),
\end{gathered}
$$

with the series converging absolutely and uniformly for $(z, g)$ in compact subsets of

$$
\{\operatorname{Re}(z)>n+1 / 2\} \times G_{n+1}\left(\mathbb{A}_{F}\right)
$$

Proof. See [44, Proposition 11.1].

6.4. Fourier-Jacobi expansions: Generalities. Let $0<r<n$ be an integer. We define the Fourier-Jacobi expansion

$$
E(f ; z, g)=\sum_{\beta \in S_{n-r}(F)} E_{\beta}(f ; z, g)
$$

where

$$
E_{\beta}(f ; z, g):=\int_{S_{n-r}(F) \backslash S_{n-r}\left(\mathbb{A}_{F}\right)} E\left(f ; z,\left(\begin{array}{ccc}
S & 0 \\
1_{n} & 0 & 0 \\
& 1_{n}
\end{array}\right) g\right) e_{F}(-\operatorname{Tr}(\beta S)) d S .
$$

LEMMA 39. Let $f=\otimes_{v} f_{v} \in I_{n}(\tau)$ be such that for some prime $v$ the support of $f_{v}$ is in $Q_{n}\left(F_{v}\right) w_{n} Q_{n}\left(F_{v}\right)$. Let $\beta \in S_{n}(F)$ and $q \in Q_{n}\left(\mathbb{A}_{F}\right)$. If $\operatorname{Re}(z)>n / 2$ then

$$
E_{\beta}(f ; z, g)=\prod \int_{v} f_{S_{n}\left(F_{v}\right)} f_{v}\left(z, w_{n} r\left(S_{v}\right) q_{v}\right) e_{v}\left(-\operatorname{Tr} \beta S_{v}\right) d S_{v} .
$$

In particular, the integrals on the right-hand side converge absolutely for $\operatorname{Re}(z)>$ $n / 2$.

Proof. See [44, Lemma 11.2]. 
Lemma 40. Suppose that $f \in I_{3}(\tau)$ and $\beta \in S_{2}(F), \beta>0$. Let $V$ be the twodimensional $\mathcal{K}$-vector space of column vectors. If $\operatorname{Re}(z)>3 / 2$ then

$$
\begin{aligned}
E_{\beta}(f ; z, g)= & \sum_{\substack{\gamma \in Q_{1}(F) \backslash G_{1}(F), \gamma \in \mathrm{U}_{1}(F)\\
}} \sum_{x \in V} \int_{S_{2}\left(\mathbb{A}_{F}\right)} f\left(w_{3}\left(\begin{array}{ccc}
1_{3} & S & x \\
& t \bar{x} & 0 \\
& 1_{3}
\end{array}\right) \alpha_{1}(1, \gamma) g\right) \\
&
\end{aligned}
$$

Recall that $e_{\mathbb{A}}(x)=e_{\mathbb{A} \mathbb{Q}}\left(\operatorname{Tr}_{F / \mathbb{Q}} x\right)$ for $x \in \mathbb{A}_{F}$.

Proof. See [44, Lemma 11.3].

We also recall a few identities which are straightforward generalizations of [44, (11.15)-(11.18)]. Letting

$$
\begin{aligned}
& F J_{\beta}(f ; z, x, g, y) \\
& \quad:=\int_{S_{2}\left(F_{v}\right)} f\left(z, w_{3}\left(\begin{array}{c}
\left.1_{n}\left(\begin{array}{cc}
S & x \\
t \bar{x} & 0
\end{array}\right)\right) \alpha_{1}\left(\operatorname{diag}\left(y,{ }^{t} y^{-1}\right), g\right) \\
1_{n}
\end{array}\right) e_{v}(-\operatorname{Tr} \beta S) d S,\right.
\end{aligned}
$$

then

$F J_{\beta}\left(f ; z, x,\left(\begin{array}{c}a \bar{a}^{-1} b \\ \bar{a}^{-1}\end{array}\right) g, y\right)=\tau_{v}^{c}(a)^{-1}|a \bar{a}|_{v}^{z+3 / 2} e_{v}\left({ }^{t} \bar{x} \beta b\right) F J_{\beta}(f ; z, x a, g, y)$.

For $u \in \mathrm{U}_{\beta}\left(\mathbb{A}_{F}\right), \mathrm{U}_{\beta}$ being the unitary group associated to $\beta$,

$$
F J_{\beta}(f ; z, x, g, u y)=\tau(\operatorname{det} u)|\operatorname{det} u \bar{u}|_{\mathbb{A}_{F}}^{-z+1 / 2} F J_{\beta}\left(f ; z, u^{-1} x, g, y\right) .
$$

If, as a function of $x, F J_{\beta}(f ; z, x, g, y) \in \mathcal{S}\left(V \otimes F_{v}\right)$, then

$$
\begin{aligned}
F J_{\beta} & \left(f ; z, x,\left(\begin{array}{cc}
a & \bar{a}^{-1} b \\
\bar{a}^{-1}
\end{array}\right) g, y\right) \\
& =\left(\lambda_{v} / \tau_{v}^{c}\right)(a)|a \bar{a}|_{v}^{z+1 / 2} \omega_{\beta}\left(\left(\begin{array}{cc}
a & \bar{a}^{-1} b \\
\bar{a}^{-1}
\end{array}\right)\right) F J_{\beta}(f ; z, x, g, y) .
\end{aligned}
$$

\subsection{Some good Siegel sections.}

6.5.1. Archimedean Siegel sections. Let $v$ be an Archimedean place of $F$. We summarize the results of $[44,11.4 .1]$. Let $\kappa \geqslant 2$ be an integer. Then $\tau(x)=$ $(x /|x|)^{-\kappa}$ is a character of $\mathbb{C}^{\times}$. 
The sections. We let $f_{\kappa, n} \in I_{n}(\tau)$ be $f_{\kappa, n}(k):=J_{n}(k, i)^{-\kappa}$. Then

$$
f_{\kappa, n}(z, q k)=J_{n}(k, i)^{-\kappa} \tau\left(\operatorname{det} D_{q}\right)\left|\operatorname{det} A_{q} D_{q}^{-1}\right|^{z+1 / 2}, \quad q \in Q_{n}(\mathbb{R}), k \in K_{n, \infty} .
$$

If $g \in \mathrm{U}_{n}(\mathbb{R})$ then $f_{\kappa, n}(z, g)=J_{n}(g, i)^{-\kappa}\left|J_{n}(g, i)\right|^{\kappa-2 z-n}$.

Fourier-Jacobi coefficients. Given a matrix $\beta \in S_{n}(\mathbb{R})$ we consider the local Fourier coefficient:

$$
f_{\kappa, n, \beta}(z, g):=\int_{S_{n}(\mathbb{R})} f_{\kappa}\left(z, w_{n}\left(\begin{array}{cc}
1_{n} & S \\
& 1_{n}
\end{array}\right) g\right) e_{\infty}(-\operatorname{Tr} \beta S) d S .
$$

This converges absolutely and uniformly for $z$ in compact subsets of $\{\operatorname{Re}(z)>$ $n / 2\}$.

LEMMA 41. Suppose that $\beta \in S_{n}(\mathbb{R})$. The function $z \mapsto f_{\kappa, \beta}(z, g)$ has a meromorphic continuation to all of $\mathbb{C}$. Furthermore, if $\kappa \geqslant n$, then $f_{\kappa, n, \beta}(z, g)$ is holomorphic at $z_{\kappa}:=(\kappa-n) / 2$, and for $y \in \mathrm{GL}_{n}(\mathbb{C}), f_{\kappa, n, \beta}\left(z_{\kappa}, \operatorname{diag}\left(y,{ }^{t} \bar{y}^{-1}\right)\right)=0$ if $\operatorname{det} \beta \leqslant 0$, and if $\operatorname{det} \beta>0$ then

$$
\begin{aligned}
f_{\kappa, n, \beta}\left(z_{\kappa}, \operatorname{diag}\left(y,{ }^{t} \bar{y}^{-1}\right)\right)= & \frac{(-2)^{-n}(2 \pi i)^{n \kappa}(2 / \pi)^{n(n-1) / 2}}{\prod_{j=0}^{n-1}(\kappa-j-1) !} \\
& \times e\left(i \operatorname{Tr}\left(\beta y^{t} \bar{y}\right)\right)(\operatorname{det} \beta)^{\kappa-n} \operatorname{det} \bar{y}^{\kappa} .
\end{aligned}
$$

Proof. See [44, Lemma 11.4].

Suppose now that $n=3$. For $\beta \in S_{2}(\mathbb{R})$ let $F J_{\beta, \kappa}(z, x, g, y):=F J_{\beta}\left(f_{\kappa} ; z, x\right.$, $g, y)$.

LEMMA 42. Let $z_{\kappa}:=(\kappa-3) / 2$. Let $\beta \in S_{2}(\mathbb{R})$, $\operatorname{det} \beta>0$.

(i) $F J_{\beta, \kappa}\left(z_{\kappa}, x, \eta, 1\right)=f_{\kappa, 2, \beta}\left(z_{\kappa}+1 / 2,1\right) e\left(i\langle x, x\rangle_{\beta}\right)$.

(ii) For $g \in \mathrm{U}_{1}(\mathbb{R})$

$$
\begin{aligned}
& F J_{\beta, \kappa}\left(z_{\kappa}, x, g, y\right)=e\left(i \operatorname{Tr} \beta y^{t} \bar{y}\right) \operatorname{det} \bar{y}^{\kappa} c(\beta, \kappa) f_{\kappa-2,1}\left(z_{\kappa}, g^{\prime}\right) \omega_{\beta}\left(g^{\prime}\right) \Phi_{\beta, \infty}(x), \\
& \text { where } g^{\prime}=\left(\begin{array}{cc}
{ }^{1}-1 &
\end{array}\right) g\left({ }_{1}{ }^{1}\right) \text { and }
\end{aligned}
$$

$$
c(\beta, \kappa)=\frac{(2 \pi i)^{2 \kappa}(2 / \pi)}{4(\kappa-1) !(\kappa-2) !} \operatorname{det} \beta^{\kappa-2},
$$

and the Weil representation $\omega_{\beta}$ is defined using the character $\lambda_{\infty}(z)=$ $(z /|z|)^{-2}$.

This is just [44, Lemma 11.5]. 
Pullback integrals. We let $\varphi$ and $F_{\kappa, z}$ be as defined in Section 4.1. The Archimedean situation is completely the same as the situation in [44]. Let $f_{\kappa} \in I_{3}(\tau)$ be as before, and let

$$
\begin{gathered}
F_{\kappa}(z, g):=\int_{\mathrm{U}_{1}(\mathbb{R})} f_{\kappa}\left(z, S^{-1} \alpha_{1}\left(g, g_{1} h\right)\right) \bar{\tau}\left(\operatorname{det} g_{1} h\right) \pi_{1}\left(g_{1} h\right) \varphi d g_{1}, \\
g \in G_{2}(\mathbb{R}), h \in G_{1}(\mathbb{R}), \mu_{1}(h)=\mu_{2}(g) .
\end{gathered}
$$

Similarly, for $f_{\kappa} \in I_{2}(\tau)$ and $g \in G_{1}(\mathbb{R})$ we let

$$
\begin{aligned}
F_{\kappa}^{\prime}(z, g) & :=\int_{\mathrm{U}_{1}(\mathbb{R})} f_{\kappa}\left(z, S^{\prime-1} \alpha_{1}^{\prime}\left(g, g_{1} h\right)\right) \bar{\tau}\left(\operatorname{det} g_{1} h\right) \pi_{1}\left(g_{1} h\right) \varphi d g_{1}, \\
g, h & \in G_{1}(\mathbb{R}), \mu_{1}(h)=\mu_{1}(g) .
\end{aligned}
$$

LEMMA 43 [44, Lemma 11.6]. The integrals converge if $\operatorname{Re}(z) \geqslant(\kappa-m-1) / 2$ and $\operatorname{Re}(z)>(m-1-\kappa) / 2, m=2$ and 1 , respectively (according to the convention of Section 6.3.1) and for such $z$ we have the following.

(i) $F_{\kappa}(z, g)=\pi 2^{-2 z-1}(\Gamma(z+(1+\kappa) / 2)) /(\Gamma(z+(3+\kappa) / 2)) F_{\kappa, z}(g)$.

(ii) $F_{\kappa}^{\prime}(z, g)=\pi 2^{-2 z}(\Gamma(z+\kappa / 2)) /(\Gamma(z+1+\kappa / 2)) \pi_{\psi}(g) \varphi$.

\subsubsection{Prime to $p$ Siegel sections: the unramified case.}

Fourier-Jacobi coefficients.

Lemma 44. Let $\beta \in S_{n}\left(F_{v}\right)$, and let $r:=\operatorname{rank}(\beta)$. Then, for $y \in \mathrm{GL}_{n}\left(\mathcal{K}_{v}\right)$,

$$
\begin{aligned}
f_{v, \beta}^{s p h}\left(z, \operatorname{diag}\left(y,{ }^{t} y^{-1}\right)\right)= & \tau(\operatorname{det} y)|\operatorname{det} y \bar{y}|_{v}^{-z+n / 2} \operatorname{Vol}\left(S_{n}\left(\mathcal{O}_{F, v}\right)\right) \\
& \times \frac{\prod_{i=r}^{n-1} L\left(2 z+i-n+1, \bar{\tau}^{\prime} \chi_{\mathcal{K}}^{i}\right)}{\prod_{i=0}^{n-1} L\left(2 z+n-i, \bar{\tau}^{\prime} \chi_{\mathcal{K}}^{i}\right)} h_{v, \bar{y} \beta y}\left(\bar{\tau}^{\prime}\left(\varpi_{v}\right) q_{v}^{-2 z-n}\right)
\end{aligned}
$$

where $h_{v, t_{\bar{y}} \beta}$ is a monic polynomial depending on $v$ and ${ }^{t} \bar{y} \beta y$ but not on $\tau$.

Proof. This is proven in [39]. See [44, Lemma 11.7].

Lemma 45. Suppose that $v$ is unramified in $\mathcal{K}$. Let $\beta \in S_{2}\left(F_{v}\right)$ be such that $\operatorname{det} \beta \neq 0$. Let $y \in \mathrm{GL}_{2}\left(\mathcal{K}_{v}\right)$ such that $\bar{y}^{t} \beta y \in S_{2}\left(\mathcal{O}_{F, v}\right)$. Let $\lambda$ be an unramified character of $\mathcal{K}_{v}^{\times}$such that $\left.\lambda\right|_{F_{v}^{\times}}=1$.

(i) If $\beta, y \in \mathrm{GL}_{2}\left(\mathcal{O}_{\mathcal{K}, v}\right)$ then for $u \in \mathrm{U}_{\beta}\left(F_{v}\right)$,

$$
F J_{\beta}\left(f_{3}^{s p h} ; z, x, g, u y\right)=\tau(\operatorname{det} u)|\operatorname{det} u \bar{u}|_{v}^{-z+1 / 2} \frac{f_{1}^{s p h}(z, g) \omega_{\beta}(u, g) \Phi_{0, y}(x)}{\prod_{i=o}^{1} L\left(2 z+3-i, \bar{\tau}^{\prime} \chi_{\mathcal{K}}^{i}\right)} \text {. }
$$


(ii) If $\bar{y}^{t} \beta y \in \mathrm{GL}_{2}\left(\mathcal{O}_{\mathcal{K}, v}\right)$, then for $u \in \mathrm{U}_{\beta}\left(F_{v}\right)$,

$$
F J_{\beta}\left(f_{3}^{s p h} ; z, x, g, u y\right)=\tau(\operatorname{det} u y)|\operatorname{det} u y|_{\mathcal{K}}^{-z+1 / 2} \frac{f_{1}^{s p h}(z, g) \omega_{\beta}(u, g) \Phi_{0, y}(s)}{\prod_{i=0}^{1} L\left(2 z+3-i, \bar{\tau}^{\prime} \chi_{\mathcal{K}}^{i}\right)} .
$$

Proof. (i) is the same as [44, Lemma 11.8]. Note that in (ii) we have removed the assumption in [44] that $g$ is of the form $\left(\begin{array}{ll}1 & \\ n & 1\end{array}\right)$. In fact since

$$
F J_{\beta}\left(f_{3}^{s p h} ; z, x, g, u y\right)=\chi(\operatorname{det} u y)|\operatorname{det} u y|_{\mathcal{K}}^{-z-1 / 2} F J_{t \bar{y} \beta y}\left(f_{3}^{s p h} ; z, y^{-1} u^{-1} x, g, 1\right)
$$

by (i) we have only to prove that

$$
\omega_{t \bar{y} \beta y}(1, g) \Phi_{0}\left(y^{-1} u^{-1} x\right)=\omega_{\beta}(u, g) \Phi_{0, y}(x)=\left(\omega_{\beta}(1, g) \Phi_{0, y}\right)\left(u^{-1} x\right),
$$

that is,

$$
\left(\omega_{t \bar{y} \beta y}(1, g) \Phi_{0}\right)_{, y}(x)=\left(\omega_{\beta}(1, g) \Phi_{0, y}\right)(x) .
$$

Here for any Schwartz function $\Phi$ we write $\Phi_{, y}$ to be the function defined by $\Phi_{, y}(x)=\Phi\left(y^{-1} x\right)$. By definition one checks that, for any $\Phi$,

$$
\omega_{\beta}(g) \Phi_{, y}=\left(\omega_{t \bar{y} \beta y}(1, g) \Phi\right)_{, y}(x)
$$

for $g$ of the forms $\left(\begin{array}{cc}a & \\ & \bar{a}^{-1}\end{array}\right),\left(\begin{array}{cc}1 & s \\ 1\end{array}\right), \eta$, thus for all $g \in \mathrm{U}_{1}\left(F_{v}\right)$. In particular, it is true for $\Phi=\Phi_{0}$.

Pullback integrals. Recall that we have $(\pi, \psi, \tau)$ as in Section 4. Let $\varphi \in V$. Let $m=1$ or 2 according to the convention of Section 6.3.1. Given $f \in I_{m+1}(\tau)$ we consider the integral

$$
F_{\varphi}(f ; z, g):=\int_{\mathrm{U}_{1}\left(F_{v}\right)} f\left(z, \gamma\left(g, g_{1} h\right)\right) \bar{\tau}\left(\operatorname{det} g_{1} h\right) \pi_{\psi}\left(g_{1} h\right) \varphi d g_{1},
$$

where $\gamma=\gamma_{1}$ or $\gamma_{1}^{\prime}$ depending on whether $m=2$ or $m=1$. (Similar to [44, 11.4].)

LEMMA 46. Suppose that $\pi, \psi$, and $\tau$ are unramified and that $\phi$ is a newvector. If $\operatorname{Re}(z)>(m+1) / 2$ then the above integral converges and

$$
F_{\varphi}\left(f_{v}^{s p h} ; z, g\right)= \begin{cases}\frac{L(\tilde{\pi}, \xi, z+1 / 2)}{\prod_{i=0}^{1} L\left(2 z+2-i, \bar{\tau}^{\prime} \chi_{\mathcal{K}}^{i}\right)} \pi_{\psi}(g) \varphi & m=1, \\ \frac{L(\tilde{\pi}, \xi, z+1)}{\prod_{i=0}^{1} L\left(2 z+3-i, \bar{\tau}^{\prime} \chi_{\mathcal{K}}^{i}\right)} F_{\rho, z}(g) & m=2 .\end{cases}
$$

Here $F_{\rho}$ is the spherical section.

Proof. This is proved in [30, Proposition 3.3]. See [44, Lemma 11.9]. 


\subsubsection{Siegel sections at ramified primes.}

The sections. Let $v$ be a finite prime of $F$. We are going to define two important Siegel sections.

(1) Let $f_{n}^{\dagger} \in I_{n}(\tau)$ be the function supported on $Q_{n}\left(\mathcal{O}_{F, v}\right) w_{n} N_{Q_{n}}\left(\mathcal{O}_{F, v}\right)$ such that

$$
f_{n}^{\dagger}\left(w_{n} r\right)=1, r \in N_{Q_{n}}\left(\mathcal{O}_{F, v}\right) \text {. }
$$

(2) Given $\left(\lambda^{u}\right) \subseteq \mathcal{O}_{\mathcal{K}, v}$ contained in the conductor of $\tau$, we let $f_{u, n} \in I_{n}(\tau)$ be the function such that $f_{u, n}(k)=\tau\left(\operatorname{det} D_{k}\right)$ if $k \in K_{Q_{n}}\left(\lambda^{u}\right)$ and $f_{u, n}(k)=0$ otherwise.

Lemma 47. Suppose that $v$ is not ramified in $\mathcal{K}$ and suppose that $\tau$ is such that $\mathcal{O}_{\mathcal{K}, v} \neq \operatorname{cond}(\tau) \supseteq \operatorname{cond}\left(\tau \tau^{c}\right)$. Let $\left(\lambda^{u}\right):=\operatorname{cond}(\tau)$. Then

$$
M\left(z, f_{n}^{\dagger}\right)=f_{u, n} \cdot \operatorname{Vol}\left(S_{n}\left(\mathcal{O}_{F, v}\right)\right) \in I_{n}\left(\bar{\tau}^{c}\right)
$$

for all $z \in \mathbb{C}$.

Proof. See [44, Lemma 11.10].

Lemma 48. Let $A \in \mathrm{GL}_{n}\left(\mathcal{K}_{v}\right)$. If $\operatorname{det} \beta \neq 0$, then

$$
\begin{aligned}
& f_{n, \beta}^{\dagger}\left(z, \operatorname{diag}\left(A,{ }^{t} \bar{A}^{-1}\right)\right) \\
& \quad= \begin{cases}\tau(\operatorname{det} A)|\operatorname{det} A|_{v}^{-z+n / 2} \operatorname{Vol}\left(S_{n}\left(\mathcal{O}_{F, v}\right)\right) & { }^{t} \bar{A} \beta A \in S_{n}\left(\mathcal{O}_{F, v}\right)^{*}, \\
0 & \text { otherwise. }\end{cases}
\end{aligned}
$$

Proof. See [44, Lemma 11.11].

Lemma 49. Suppose that $\beta \in S_{n}\left(F_{v}\right), \operatorname{det} \beta \neq 0, \operatorname{char}(v)=\ell$, and $\ell$ splits completely in $\mathcal{K}$.

(i) If $\beta \notin S_{n}\left(\mathcal{O}_{F, v}\right)$, then $M\left(z, f_{n}^{\dagger}\right)_{\beta}(-z, 1)=0$.

(ii) Suppose that $\beta \in S_{n}\left(\mathcal{O}_{F, v}\right)$. Let $c:=\operatorname{ord}_{v}\left(\operatorname{cond}\left(\tau^{\prime}\right)\right)$. If $c>0$, then

$$
M\left(z, f_{n}^{\dagger}\right)_{\beta}(-z, 1)=\tau^{\prime}(\operatorname{det} \beta)|\operatorname{det} \beta|_{v}^{-2 z} g\left(\bar{\tau}^{\prime}\right)^{n} c_{n}\left(\tau^{\prime}, z\right),
$$

where

$$
c_{n}\left(\tau^{\prime}, z\right)= \begin{cases}\chi^{\prime}\left(\varpi_{v}^{n c}\right) q_{v}^{2 n c z-c n(n+1) / 2} & c>0 \\ q_{v}^{2 n z-n(n+1) / 2} & c=0 .\end{cases}
$$


Proof. See [44, Lemma 11.12].

Now we use the convention for $m=1$ or 2 in Section 6.3.1.

PROPOSITION 50. Let $m=1$ or 2. There exists a meromorphic function $\gamma^{(m)}(\rho, z)$ on $\mathbb{C}$ such that the following hold.

(i) If $m=1$, then

$$
F_{\varphi \vee}(M(z, f) ;-z, g)=\gamma^{(1)}(\rho, z) \tau\left(\mu_{1}(g)\right) F_{\varphi}(f ; z, \eta g) .
$$

Moreover, if $\pi \simeq \pi\left(\chi_{1}, \chi_{2}\right)$ and $v$ splits in $\mathcal{K}$, then

$$
\begin{aligned}
\gamma^{(1)}(\rho, z)= & \psi(-1) g\left(\bar{\tau}^{\prime}, \varpi_{v}^{c}\right)^{2} \cdot \tau^{\prime}\left(\varpi_{v}^{2 c}\right)\left|\varpi_{v}\right|_{v}^{-4 c z+3 c} \\
& \times \epsilon\left(\tilde{\pi} \otimes \xi^{c}, z+1 / 2\right) \frac{L\left(\pi \otimes \xi^{c}, 1 / 2-z\right)}{L\left(\tilde{\pi} \otimes \xi^{c}, z+1 / 2\right)} .
\end{aligned}
$$

(ii) If $m=2$ and $\pi, \psi, \tau$ are the $v$ constituents of a global triple, $\varphi^{\vee}=\pi(\eta) \varphi$, then

$$
F_{\varphi^{\vee}}(M(z, f) ;-z, g)=\gamma^{(2)}(\rho, z) A\left(\rho, z, F_{\varphi}(f ; z,-)\right)_{-z}(g) .
$$

Each of these equalities is an identity of meromorphic functions of $z$.

(iii) Suppose moreover that $\mathcal{O}_{v} \neq \operatorname{cond}(\tau) \supset \operatorname{cond}\left(\tau \tau^{c}\right)$. Then

$$
\gamma^{(2)}(\rho, z)=\gamma^{(1)}\left(\rho, z-\frac{1}{2}\right) .
$$

Proof. See [44, Lemma 11.12].

\subsubsection{Sections at ramified primes again.}

The sections. Again let $v$ be a finite prime of $F$. As in [44, 11.4.14], we define a modified version of the sections $f^{\dagger}$. Let $m=1$ or 2 . For $x \in \mathcal{O}_{\mathcal{K} . v} \cap \mathcal{K}_{v}^{\times}$let

$$
f_{x}^{\dagger,(m)}(z, g)=f_{m+1}^{\dagger}\left(z, g\left(\begin{array}{cccccc}
1 & & & & & 1 / x \\
& 1_{m-1} & & & 0_{m-1} & \\
& 1 & 1 / \bar{x} & & \\
& & 1_{m-1} & & \\
& & & 1 & \\
& & & & & 1
\end{array}\right)\right) \text {. }
$$


Fourier-Jacobi coefficients.

LEMMA 51. Let $\beta=\left(b_{i, j}\right) \in S_{m+1}\left(F_{v}\right)$. Then, for all $z \in \mathbb{C}, f_{x, \beta}^{\dagger,(m)}(z, 1)=0$ if $\beta \notin S_{m+1}\left(\mathcal{O}_{F, v}\right)^{*}$. If $\beta \in S_{m+1}\left(\mathcal{O}_{F, v}\right)^{*}$, then

$$
f_{x, \beta}^{\dagger,(m)}(z, 1)=\operatorname{Vol}\left(S_{m+1}\left(\mathcal{O}_{F, v}\right)\right) e_{v}\left(\operatorname{Tr}_{\mathcal{K}_{v} / F_{v}}\left(b_{m+1,1} / x\right)\right) .
$$

Proof. See [44, Lemma 11.14].

LEMmA 52. Let $\beta \in S_{2}\left(F_{v}\right)$, $\operatorname{det} \beta \neq 0$. Let $y \in \mathrm{GL}_{2}\left(\mathcal{K}_{v}\right)$, and suppose that ${ }^{t} \bar{y} \beta y \in S_{2}\left(\mathcal{O}_{F, v}\right)^{*}$. Let $\lambda, \theta$ be characters of $\mathcal{K}_{v}^{\times}$, and suppose that $\left.\lambda\right|_{F_{v}^{\times}}=1$. Let $(c):=\operatorname{cond}(\lambda) \bigcap \operatorname{cond}(\theta) \bigcap\left(\varpi_{v}\right)$. Let $x \in \mathcal{K}_{v}^{\times}$be such that $D_{v} \mid x$, cond $\left(\chi^{c}\right) \mid x$, and $c D_{v}$ det $\bar{y}^{t} \beta y \mid x$, where $D_{v}:=\operatorname{Nm}_{\mathcal{K} / F}\left(\delta_{\mathcal{K} / \mathbb{Q}}\right)$. Suppose that $y^{-1} \beta^{-1 t} \bar{y}^{-1}=\left(\begin{array}{c}* * \\ *\end{array}\right)$ with $d \in F_{v}$. For $\tilde{D}_{v}:=\operatorname{Nm}_{\mathcal{K} / F}\left(\delta_{\mathcal{K} / F}\right)$ then, for $h \in U_{\beta}\left(F_{v}\right)$,

$$
\begin{aligned}
& \sum_{a \in\left(\mathcal{O}_{v} / x\right)^{\times}} \theta \bar{\tau}^{c}(a) F J_{\beta}\left(f_{x}^{\dagger,(2)} ; z, u, g\left(\begin{array}{rr}
a^{-1} & \\
& \bar{a}
\end{array}\right), h y\right) \\
& =\tau(\operatorname{det} h y)|\operatorname{det} h y|_{\mathcal{K}}^{-z+1 / 2} \operatorname{Vol}\left(S_{2}\left(\mathcal{O}_{F, v}\right)\right)
\end{aligned}
$$

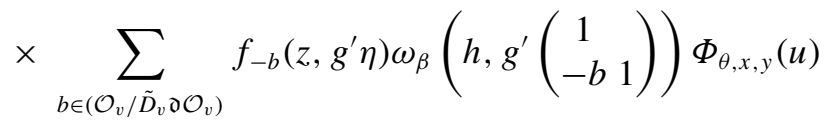

where $g^{\prime}=\left(\begin{array}{ll}1^{1} & \end{array}\right) g\left(\begin{array}{ll}1_{-1}\end{array}\right)$ and

$$
f_{b}(g)= \begin{cases}\tau \lambda^{-1}\left(d_{p}\right), & g=p \eta\left(\begin{array}{c}
1 \\
m \\
1
\end{array}\right), p \in B_{1}\left(\mathcal{O}_{F, v}\right), m-b \in \tilde{D}_{v} \mathfrak{d} \mathcal{O}_{F, v} \\
0 & \text { otherwise. }\end{cases}
$$

Proof. The proof is the same as that of [44, Lemma 11.15].

Pullback integrals. Let $\mathcal{T}$ denote a triple $(\varphi, \psi, \tau)$ with $\varphi \in V$ having a conductor with respect to $\tilde{\pi}$. Let

$$
\varphi_{x}:=\pi_{\psi}\left(\eta \operatorname{diag}\left(\bar{x}^{-1}, x\right)\right) \psi
$$

and let

$$
F_{\mathcal{T}, x}^{(m)}(z, g):=\int_{\mathrm{U}_{1}\left(F_{v}\right)} f_{x}^{\dagger,(m)}\left(z, S^{-1} \alpha\left(g, g^{\prime} h\right)\right) \bar{\tau}\left(\operatorname{det} g^{\prime} h\right) \pi_{\psi}\left(g^{\prime} h\right) \varphi_{x} d g^{\prime},
$$

where $\alpha=\alpha_{1}$ or $\alpha_{1}^{\prime}$ depending on whether $m=2$ or 1 . If $f(z, g)=f_{x}^{\dagger,(m)}$ $\left(z, g S^{-1}\right)$ then $F_{\mathcal{T}, x}^{(m)}(z, g)=F_{\varphi_{x}}(f ; z, g)$. 
Proposition 53. Suppose that $x=\lambda^{t}, t>0$ is contained in the conductors of $\tau$ and $\psi$ and $x \bar{x} \in\left(\lambda^{r_{\phi}}\right)=\operatorname{cond}_{\tilde{\pi}}(\varphi)$. Then $F_{\mathcal{T}, x}^{(m)}(z, g)$ converges for all $z$ and $g$ and

$$
F_{\mathcal{T}, x}^{(1)}(z, \eta)=\left[\mathrm{U}_{1}\left(\mathcal{O}_{F, v}\right): K_{x}\right]^{-1} \tau(x)|x \bar{x}|_{v}^{-z-1} \varphi
$$

and

$$
F_{\mathcal{T}, x}^{(2)}=\left[\mathrm{U}_{1}\left(\mathcal{O}_{F, v}\right): K_{x}\right]^{-1} \tau(x)|x \bar{x}|_{v}^{-z-3 / 2} F_{\varphi, r, t}
$$

for any $r \geqslant \max \left\{r_{\varphi}, t\right\}$. Here $K_{x}$ is the subgroup defined as

$$
K_{x}:=\left\{\left(\begin{array}{ll}
a & b \\
c & d
\end{array}\right) \in \mathrm{U}_{1}\left(\mathcal{O}_{F, v}\right): a-1 \in(\bar{x}), b \in(x \bar{x}), c \in \mathcal{O}_{v}, d-1 \in(x)\right\} .
$$

Proof. See [44, Proposition 11.16].

Proposition 54. For $m=1$ or 2 , let $\gamma^{(m)}(\rho, z)$ be as in Proposition 50. Assume that $\operatorname{char}(v)=\ell$, which is unramified in $\mathcal{K}$. If $\mathcal{O}_{v} \neq \operatorname{cond}(\tau) \supseteq \operatorname{cond}\left(\tau \tau^{c}\right)$ then

$$
\gamma^{(2)}(\rho, z)=\gamma^{(1)}(\rho, z-1 / 2) \text {. }
$$

Proof. See [44, Proposition 11.17].

6.5.5. p-adic sections. Now let $v \mid p$ be a prime of $F$. We record the formulas for Fourier-Jacobi coefficients and pullback sections below (see [50] for the discussion). These are only slightly different from $[44,11.4]$ (the $\xi^{c}$ that appeared there are replaced by $\xi^{c} \mu_{1, v}^{-1}$ in our cases). Let $x \in \mathcal{O}_{\mathcal{K}, v}$ be such that $(x)=$ $\operatorname{cond}\left(\bar{\xi}^{c}\right)$. We define

$$
f_{z}^{0,(m)}(k):=\psi(\operatorname{det} k) \bar{\psi}^{m+1}\left(\mu_{m+1}(k)\right) M\left(z, f_{x}^{\dagger,(m)}(k)\right) .
$$

LEMMA 55. Suppose that our data (Section 4.1.3) is in the generic case, and let $\left(p^{m}\right):=\operatorname{cond}\left(\tau^{\prime}\right)$. Let $\beta \in S_{2}\left(F_{v}\right)$, det $\beta \neq 0$, and suppose that $\beta \in \mathrm{GL}_{2}\left(\mathcal{O}_{v}\right)$. Let $y \in \mathrm{GL}_{2}\left(\mathcal{O}_{v}\right)$. Let $\lambda$ be an unramified character of $\mathcal{K}_{v}^{\times}$such that $\left.\lambda\right|_{F_{v}^{\times}}=1$. Then, for $h \in \mathrm{U}_{\beta}\left(F_{v}\right)$,

$$
\begin{aligned}
& \sum_{a \in\left(\mathcal{O}_{v} / x\right)^{\times}} \mu_{1, v}^{-1} \xi^{c} \tau(a) F J_{\beta}\left(f_{-z}^{0,(2)} ; z, u, g \operatorname{diag}\left(a^{-1}, \bar{a}\right), h y\right) \\
& \quad=\xi(-1) c(\beta, \tau, z) \tau(\operatorname{det} h y)|\operatorname{det} h \bar{h}|_{v}^{-z+1 / 2} f_{m, 1}(z, g \eta) \omega_{\beta}(h, g) \Phi_{\mu_{1, v}^{-1} \xi^{c}, x, y}(u),
\end{aligned}
$$

where $\omega$ is defined using $\lambda$, and

$$
c(\beta, \tau, z):=\bar{\tau}^{\prime}(-\operatorname{det} \beta)|\operatorname{det} \beta|_{v}^{2 z+1} \mathfrak{g}\left(\tau^{\prime}\right)^{2} \bar{\tau}^{\prime}\left(p^{2 m}\right) p^{-(4 m z+5 m) e_{v}},
$$

where $e_{v}$ is $\left[F_{v}: \mathbb{Q}_{p}\right]$. 
Proof. See [44, Lemma 11.20]. The argument in [44] still works as long as $F_{v}$ is unramified.

Now we use the convention for $m=1$ or 2 as in Section 6.5.2.

Proposition 56. Let $\varphi \in V$ be an eigenvector for $\pi$ such that $v \mid \operatorname{cond}_{\pi}(\varphi)$. Let

$$
(x):=\operatorname{cond}\left(\bar{\xi}^{c}\right)=\left(\varpi_{v}^{t}\right)=\left(\varpi_{v}^{t_{1}}, \varpi_{v}^{t_{2}}\right) .
$$

Suppose again that we are in the generic case. Let

$$
\varphi_{x}^{\vee}:=\psi(-1) \pi\left(\operatorname{diag}\left(x, \bar{x}^{-1}\right)\right) \varphi .
$$

Then

$$
\begin{aligned}
& F_{\varphi_{x}^{\vee}}\left(\tilde{f}_{-z}^{0,(m)} ; z, g\right) \\
& \quad=\gamma^{(m)}\left(\rho^{\vee},-z\right)\left[U_{1}\left(\mathcal{O}_{F, v}\right): K_{x}\right]^{-1} \bar{\tau}^{c}(x)|x \bar{x}|_{v}^{z-(m+1) / 2} \begin{cases}F_{\varphi, z}^{0}(g), & m=2, \\
\pi_{\psi}(g) \varphi, & m=1,\end{cases}
\end{aligned}
$$

where $\tilde{f}_{-z}^{0,(m)}(z, g)=f_{-z}^{0, m}\left(z, g S^{-1}\right)$.

Proof. The proof is the same as that of [44, Proposition 11.21].

6.6. Good Siegel Eisenstein series. From now on we assume that the characters $\psi$ and $\tau$ are unramified outside $p$. Let $(\pi, V)=\left(\otimes \pi_{v}, \otimes V_{v}\right)$ be as before, and let $\mathcal{D}=(\Sigma, \varphi, \psi, \tau)$ be an Eisenstein datum as defined in Section 4.3. We augment the datum with a choice of an $M_{\mathcal{D}} \in \mathbb{A}_{F}^{\times}$satisfying the following.

- $M_{\mathcal{D}}$ is divisible only by primes in $\Sigma \backslash\{v \mid p\}$.

- For $v \in \Sigma \backslash\{v \mid p\}, M_{\mathcal{D}}$ is contained in $\delta_{\mathcal{K}}$, cond $\left(\xi_{v}\right)$, cond $\left(\psi_{v}\right)$, cond $\left(\tau_{v}\right)$, and $\operatorname{cond}_{\pi_{v}^{\vee}}\left(\varphi_{v}\right)$.

We remark that we have freedom to choose such $M_{\mathcal{D}}$. This is crucial for proving the $p$-adic properties of the Eisenstein series.

The Siegel Eisenstein series. For $m=1$ or 2 we define a meromorphic section $f_{\mathcal{D}}^{(m)}: \mathbb{C} \rightarrow I_{m+1}(\tau)$ as follows:

$$
f_{\mathcal{D}}^{(m)}(z)=\otimes f_{\mathcal{D}, w}^{(m)}(z)
$$

where

- $f_{\infty}^{(m)}(z):=f_{\kappa} \in I_{m+1}\left(\tau_{\infty}\right)$ for any infinite place;

- $v \nmid \Sigma$ then $f_{\mathcal{D}, v}^{(m)}(z):=f_{v}^{s p h} \in I_{m+1}\left(\tau_{v}\right)$; 
- if $v \in \Sigma, v \nmid p$, then $f_{\mathcal{D}, v}^{(m)}(z):=f_{M_{\mathcal{D}}, v}^{(m)} \in I_{m+1}\left(\tau_{v}\right)$; and

- for $v \mid p, f_{\mathcal{D}, v}^{(m)}(z):=f_{-z}^{0,(m)} \in I_{m+1}\left(\tau_{v}\right)$, where $x_{v}$ is used to define $f_{-z}^{0,(m)}$.

DEFINITION 57. $H_{\mathcal{D}}^{(m)}(z, g):=E\left(f_{\mathcal{D}}^{(m)} ; z, g\right)$.

Now we define a level group for $\mathrm{U}(1,1)$ :

$$
U_{\mathcal{D}}:=\prod_{v \mid p} K_{x_{v}, v} \prod_{v \notin \Sigma \backslash\{v \mid p\}} K_{M_{\mathcal{D}}, v} \prod_{v \notin \Sigma} \mathrm{U}_{1}\left(\mathcal{O}_{F, v}\right),
$$

with $K_{x_{v}, v}$ defined in Proposition 53.

REMARK 58. Later on we will use $U_{\mathcal{D}}$ to denote the corresponding level groups in $\mathrm{GL}_{2}$ as well.

We also let

$$
K_{\mathcal{D}}^{(m)}:=\left\{k \in G_{m+1}\left(\hat{\mathcal{O}}_{F}\right): 1-k \in M_{\mathcal{D}}^{2} \prod_{v \mid p}\left(x_{v} \bar{x}_{v}\right) \cdot M_{2(m+1)}\left(\hat{\mathcal{O}}_{F}\right)\right\} .
$$

Then it easily follows from the definition of the $f_{\mathcal{D}, v}^{(m)}(z)$ that

$$
H_{\mathcal{D}}^{(m)}(z, g k)=H_{\mathcal{D}}^{(m)}(z, g), \quad k \in K_{\mathcal{D}}^{(m)},
$$

and that

$$
H_{\mathcal{D}}^{(m)}(z, g \alpha(1, k))=\tau\left(a_{k_{p}}\right) H_{\mathcal{D}}^{(m)}(z, g), \quad k \in U_{\mathcal{D}}
$$

For $u \in \mathrm{GL}_{m+1}\left(\mathbb{A}_{\mathcal{K}, f}\right)$ let

$$
L_{v}^{(m)}:=\left\{\beta \in S_{m+1}(F): \beta \geqslant 0, \operatorname{Tr} \beta \gamma \in \hat{\mathcal{O}}_{F}, \gamma \in u S_{m+1}\left(\hat{\mathcal{O}}_{F}\right)^{t} \bar{u}\right\} .
$$

We record the following formulas, which are slight generalizations of the results in $[44,11.5]$. These will be used to construct the $p$-adic families of $L$ values and Eisenstein series.

LEMMA 59. (i) If $\kappa \geqslant m+1$, then $H_{\mathcal{D}}^{(m)}$ is holomorphic at $z_{\kappa}:=(\kappa-m-1) / 2$.

(ii) If $\kappa \geqslant m+1$ and if $g \in Q_{m+1}\left(\mathbb{A}_{F}\right)$, then

$$
H_{\mathcal{D}}^{m}\left(z_{\kappa}, g\right)=\sum_{\beta \in S_{m+1}(F), \beta>0} H_{\mathcal{D}, \beta}^{(m)}\left(z_{\kappa}, g\right) .
$$


Furthermore, if $S_{2}(F) \ni \beta \gg 0, g_{\infty, i}=r\left(X_{i}\right) \operatorname{diag}\left(Y_{i},{ }^{t} \bar{Y}_{i}^{-1}\right)$ for the ith Archimedean place and $g_{f}=r(a) \operatorname{diag}\left(u,{ }^{t} \bar{u}^{-1}\right) \in G_{m+1}\left(\mathbb{A}_{F, f}\right)$, then $H_{\mathcal{D}, \beta}^{(m)}\left(z_{\kappa}, g\right)$ $=0$ if $\beta \notin L_{u}^{(m)}$, and otherwise

$$
\begin{aligned}
& H_{\mathcal{D}, \beta}^{(m)}\left(z_{k}, g\right) \\
& =e(\operatorname{tr} \beta a) \frac{(-2)^{-(m+1) d}(2 \pi i)^{(m+1) d \kappa}(2 / \pi)^{m(m+1) d / 2} \prod_{j}\left(\operatorname{det} \beta_{j}^{\kappa-(m+1)} \cdot \operatorname{det} \bar{Y}_{j}^{\kappa}\right)}{\left(\prod_{j=0}^{m}(\kappa-j-1) !\right)^{d} \prod_{j=0}^{m} L^{S}\left(\kappa-j, \bar{\tau}^{\prime} \chi_{\mathcal{K}}^{j}\right)} \\
& \quad \times \prod_{j \in I} e\left(\operatorname{Tr} \beta_{j}\left(X_{j}+i Y_{j} \bar{Y}_{j}^{t}\right)\right) \prod_{v \notin S} f_{\mathcal{D}, \beta_{u}, v}\left(z_{\kappa}, 1\right) \\
& \quad \times \tau(\operatorname{det} u)|\operatorname{det} u \bar{u}|_{F}^{m+1-\kappa / 2} \prod_{v \notin S} h_{v, \beta}\left(\bar{\tau}_{v}^{\prime}\left(\varpi_{v}\right) q_{v}^{-2 z-n}\right),
\end{aligned}
$$

where $\beta_{u}={ }^{t} \bar{u} \beta u, \beta_{j}=\iota_{j}(\beta), \iota_{j}$ is the $j$ th embedding $F \hookrightarrow \mathbb{R}$, and $S \supseteq \Sigma$ is a finite set of primes such that $g_{v} \in K_{m+1, v}$ if $v \notin S$.

Proof. The proof is the same as that of [44, Lemma 11.22].

If $\kappa \geqslant m+1$, define a function $H_{\mathcal{D}}^{m}(Z, x)$ on $\mathbf{H}_{m+1}^{\Sigma_{\infty}} \times G_{m+1}\left(\mathbb{A}_{F, f}\right)$ by $H_{\mathcal{D}}^{(m)}(Z, x):=\prod_{j=1}^{d} \mu_{m+1}\left(g_{\infty, j}\right)^{(m+1) \kappa / 2} \prod_{j=1}^{d} J_{m+1}\left(g_{\infty, j}, i\right)^{-\kappa} H_{\mathcal{D}}^{(m)}\left((\kappa-m-1) / 2, g_{\infty} x\right)$, where $g_{\infty} \in G_{m+1}^{+}(\mathbb{R}), g_{\infty}(i)=Z$, and define $A_{\mathcal{D}, \beta}^{(m)}(x)$ as the $\beta$ th Fourier coefficient of $H_{\mathcal{D}}^{(m)}(Z, x)$.

LEMma 60. Suppose that $\kappa \geqslant m+1$. Then $H_{\mathcal{D}}^{(m)}(Z, x) \in M_{\kappa}\left(K_{\mathcal{D}}^{(m)}\right)$ (notation as in Section 2.3, where $\kappa$ stands for the scalar weight $\underline{k}:=(0, \ldots, 0 ; \kappa, \ldots, \kappa))$.

Proof. The proof is the same as that of [44, Lemma 11.23].

LEMma 61. Suppose that $\kappa \geqslant m+1$ and that $x=\operatorname{diag}\left(u,{ }^{t} \bar{u}^{-1}\right), u \in$ $\mathrm{GL}_{m+1}\left(\mathbb{A}_{F, f}\right)$ with $u_{v}=\operatorname{diag}\left(1_{m}, \bar{a}_{v}\right), a_{v} \in \mathcal{O}_{v}^{\times}$, if $v \in \Sigma$. Then, if $\beta \notin L_{u}^{(m)}$ or if $\operatorname{det} \beta=0$, then $A_{\mathcal{D}, \beta}(x)=0$, and for $\beta=\left(\beta_{i, j}\right) \in L_{u}^{(m)}$ with $\operatorname{det} \beta>0$,

$$
\begin{aligned}
A_{\mathcal{D}, \beta}^{(m)}(x)= & \left|\delta_{\mathcal{K}}\right|_{\mathcal{K}}^{m(m+1) / 4}\left|\delta_{F}\right|_{F}^{(m+1) / 2} \\
& \times \frac{(-2)^{-(m+1) d}(2 \pi i)^{(m+1) \kappa d}(2 / \pi)^{m(m+1) d / 2} \prod_{v \mid p}\left(\operatorname{det} \beta|\beta|_{v}\right)^{\kappa-m-1}}{\prod_{j=0}^{m}(\kappa-j-1) !^{d} \prod_{j=0}^{m} L^{\Sigma}\left(\kappa-j, \bar{\tau}^{\prime} \chi_{\mathcal{K}}^{j}\right)} \\
& \times \prod_{v \mid p} \bar{\tau}_{v}\left(a_{v} \operatorname{det}(\beta)\right) \mathfrak{g}\left(\tau_{v}^{\prime}\right)^{m+1} c\left(\bar{\tau}_{v}^{\prime},-(\kappa-m-1) / 2\right) e_{v}\left(\operatorname{Tr}_{\left.\mathcal{K}_{v} / \mathbb{Q}_{v}\left(a_{v} b_{m+1,1} / x_{v}\right)\right)}\right.
\end{aligned}
$$




$$
\begin{aligned}
& \times \prod_{v \in \Sigma, v \nmid p} \tau_{v}^{c}\left(a_{v}\right) e_{v}\left(\operatorname{Tr}_{\mathcal{K}_{v} / \mathbb{Q}_{v}}\left(a_{v} b_{m+1,1} / M_{\mathcal{D}}\right)\right) \\
& \times \prod_{v \notin \Sigma} \tau_{v}\left(\operatorname{det} u_{v}\right)\left|u_{v} \bar{u}_{v}\right|_{v}^{m+1-\kappa / 2} h_{v, \bar{u}_{v}^{t} \beta u_{v}}\left(\bar{\tau}_{v}\left(\varpi_{v}\right) q_{v}^{-\kappa}\right) .
\end{aligned}
$$

Proof. See [44, Lemma 11.24], taking into account the $\operatorname{Vol} S_{n}\left(\mathcal{O}_{F, v}\right)$.

6.7. $\boldsymbol{E}_{\mathcal{D}}$ via pull-back. For a normalized Hilbert modular eigenform $f \in \pi$ we write $\varphi:=\pi\left(\eta^{M}\right) f$, where $\eta_{v}^{M}=\eta$ if $v \mid M$ and is 1 otherwise. As in $[44,11.6]$ we let $\varphi_{0}$ be defined by $\varphi_{0}(g)=\varphi_{\psi}(g y)$ for

$$
y_{v}= \begin{cases}1, & v=\infty, v \notin \Sigma, \\ \eta^{-1} \operatorname{diag}\left(M_{\mathcal{D}}^{-1}, M_{\mathcal{D}}\right) & v \in \Sigma, v \nmid p, \\ \operatorname{diag}\left(x_{v}, \bar{x}_{v}^{-1}\right), & v \mid p .\end{cases}
$$

Here $\varphi \in V$ and $\varphi_{\psi}$ is the form on $\operatorname{GU}(1,1)\left(\mathbb{A}_{F}\right)$ given by $\varphi$ and $\psi$.

PROPOSITION 62. Let $m=1$ or 2. Suppose that, for any $v \mid p,\left(x_{v}\right)=\left(p^{t_{v}}\right)$ with $t_{v}>0$, and that $x_{v} \in \operatorname{cond}(\psi)$ and $x_{v} \bar{x}_{v} \in \operatorname{cond}_{\pi_{v}}\left(\varphi_{v}\right)$, where $\varphi_{v}$ is defined by $\varphi=\otimes \varphi_{v}$. Let $g \in G_{m}\left(\mathbb{A}_{F}\right)$ and $h \in G_{1}\left(\mathbb{A}_{F}\right)$ be such that $\mu_{1}(h)=\mu_{m}(g)$. If $k \geqslant m+1$ then

$$
\begin{gathered}
\int_{\mathrm{U}_{1}(F) / \mathrm{U}_{1}\left(\mathbb{A}_{F}\right)} H_{\mathcal{D}}^{(m)}\left(z, \alpha\left(g, g^{\prime} h\right)\right) \bar{\tau}\left(\operatorname{det} g^{\prime} h\right) \varphi_{0}\left(g^{\prime} h\right) d g^{\prime} \\
=\left[\mathrm{U}_{1}\left(\hat{\mathcal{O}}_{F}\right): U_{\mathcal{D}}\right]^{-1} \begin{cases}c_{\mathcal{D}}^{(1)}(z) \varphi(g) & m=1 \\
c_{\mathcal{D}}^{(2)}(z) E_{\mathcal{D}}(z, g) & m=2,\end{cases}
\end{gathered}
$$

where

$$
\begin{aligned}
c_{\mathcal{D}}^{(m)}(x):= & \pi^{d} 2^{(-2 z-m+1) d}\left|M_{\mathcal{D}}\right|_{F}^{(2 z+m+1)} \prod_{v \mid p}\left|x_{v} \bar{x}_{v}\right|_{v}^{z-(m+1) / 2} \bar{\tau}_{v}^{c}\left(x_{v}\right) \prod_{v \nmid p} \tau_{v}\left(M_{\mathcal{D}}\right) \\
& \times \frac{\Gamma(z+(m-1+\kappa) / 2)^{d} L^{\Sigma}(\tilde{\pi}, \xi, z+m / 2)}{\Gamma(z+(m+1+\kappa) / 2)^{d} \prod_{i=0}^{1} L^{\Sigma}\left(\bar{\tau}^{\prime} \epsilon_{\mathcal{K}}^{i}, 2 z+m+1-i\right)} \\
& \times \prod_{v \mid p} \gamma^{(m)}\left(\rho_{1, v},-z\right) .
\end{aligned}
$$

This is just a summary of the previous computations, similar to [44, Proposition 11.25]. We also have the following result for Fourier coefficients which is an immediate consequence of the above proposition. 
Proposition 63. Let $m=1$ or 2. Suppose that, for each $v \mid p,\left(x_{v}\right)=\left(p^{t_{p}}\right)$ with $t_{p}>0$, and that $x_{v} \in \operatorname{cond}(\psi)$ and $x_{v} \bar{x}_{v} \in \operatorname{cond}_{\pi_{v}}\left(\phi_{v}\right)$. Let $g \in G_{m}\left(\mathbb{A}_{F}\right)$ and $h \in G_{1}\left(\mathbb{A}_{F}\right)$ be such that $\mu_{1}(h)=\mu_{m}(g)$. Let $\beta \in S_{m}(F)$. If $\kappa \geqslant m+1$ then

$$
\begin{array}{r}
\int_{\mathrm{U}_{1}(F) / \mathrm{U}_{1}\left(\mathbb{A}_{F}\right)} H_{\mathcal{D}, \beta}^{(m)}\left(z, \alpha\left(g, g^{\prime} h\right)\right) \bar{\tau}\left(\operatorname{det} g^{\prime} h\right) \varphi_{0}\left(g^{\prime} h\right) d g^{\prime} \\
=\left[U_{1}\left(\hat{\mathcal{O}}_{F}\right): U_{\mathcal{D}}\right]^{-1} \begin{cases}c_{\mathcal{D}}^{(1)}(z) \varphi_{\beta}(g) & m=1, \\
c_{\mathcal{D}}^{(2)}(z) \mu_{\mathcal{D}}(\beta, z, g) & m=2,\end{cases}
\end{array}
$$

where $c_{\mathcal{D}}^{(m)}(z)$ is as defined above.

Recall that $a_{1}, \ldots, a_{h_{\mathcal{K}}} \in \hat{\mathcal{O}}_{\mathcal{K}}$ are representatives for the class group of $\mathcal{K}$. We assume that each $a_{i}=\left(\varpi_{v}, 1\right) \in \mathcal{O}_{\mathcal{K}, v}$ for some prime $v \notin \Sigma$ that splits in $\mathcal{K}$. Let

$$
\Gamma_{\mathcal{D}}: \mathrm{U}_{1}(F) \cap U_{\mathcal{D}}, \quad \Gamma_{\mathcal{D}, i}:=\mathrm{U}_{1}(F) \cap\left(\begin{array}{cc}
a_{i}^{-1} & \\
& \bar{a}_{i}
\end{array}\right) U_{\mathcal{D}}\left(\begin{array}{cc}
a_{i} & \\
& \bar{a}_{i}^{-1}
\end{array}\right) .
$$

Also, we write $\Gamma_{\mathcal{D}, 0} \supseteq \Gamma_{\mathcal{D}}$ by removing the congruence conditions required for diagonal entries (similar to $\Gamma_{0}(N) \supset \Gamma_{1}(N)$ in the classical case). We often write $\Gamma_{\mathcal{D}, 0}$ for the $\mathrm{GL}_{2}\left(\mathbb{A}_{F, f}\right)$ open compact group with the same congruence requirement as for $\mathrm{U}_{1}$ (that is putting the same congruence conditions on $c$ for $\left.\left(\begin{array}{ll}a & b \\ c & d\end{array}\right)\right)$. For any $v \mid p$ let

$$
\left(p^{u_{v}}\right):=\left(x_{v}\right) \cap \mathcal{O}_{F, v},\left(p^{r_{v}}\right)_{v}:=\left(x_{v} \bar{x}_{v}\right) .
$$

It follows easily from the strong approximation that if we let $\mathcal{Y} \subset \hat{\mathcal{O}}$ be any set of representatives for $\left(\hat{\mathcal{O}}_{\mathcal{K}} / \prod_{v \mid p} \bar{x}_{v} M_{\mathcal{D}}\right)^{\times} /\left(\hat{\mathcal{O}}_{F} / p^{u_{p}} M_{\mathcal{D}}\right)^{\times}$, then

$$
\mathrm{U}_{1}\left(\mathbb{A}_{F}\right)=\bigsqcup_{i=1}^{h_{\mathcal{K}}} \bigsqcup_{a \in \mathcal{Y}} \mathrm{U}_{1}(F) \mathrm{U}_{1}\left(F_{\infty}\right)\left(\begin{array}{cc}
a_{i}^{-1} a^{-1} & \\
& \bar{a}_{i} \bar{a}
\end{array}\right) U_{\mathcal{D}}
$$

with each element appearing $2^{v_{\mathcal{K}}} h_{F}$ times, where $v_{\mathcal{K}}$ is a number depending only on $\mathcal{K}$. Define

$$
\tilde{H}_{\mathcal{D}, \beta}^{(m)}(z, g):=\sum_{a \in\left(\hat{\mathcal{O}}_{\mathcal{K}} /\left(\left(\prod_{v \mid p} x_{v}\right) M_{\mathcal{D}}\right)\right)^{\times}}\left(\prod_{v \mid p} \mu_{1, v}^{-1}\right) \xi^{c} \tau(a) H_{\mathcal{D}, \beta}^{(m)}\left(z, g \alpha\left(1, \operatorname{diag}\left(a^{-1}, \bar{a}\right)\right)\right) .
$$

6.8. Nebentypus. In this subsection we discuss the relations between $\mathrm{U}(1,1)$ automorphic forms and $\mathrm{GL}_{2}$ automorphic forms. In the [44] case the situation is easier since they assumed that the forms are newforms, that is, invariant under the 
action of matrices: $\left(\begin{array}{c}x \\ y \\ 1\end{array}\right)$ for $x \in \mathcal{O}_{F, v}^{\times}, y \in \mathcal{O}_{F, v}$. Since we are going to work with the full-dimensional Hida family we do not assume this anymore. A principle for this issue is that we assume the neben characters at places not dividing $p$ and the torsion part at $p$-adic places to be invariant under the action of matrices: $\left(\begin{array}{cc}x & y \\ 1\end{array}\right)$ for $x \in \mathcal{O}_{F, v}^{\times}, y \in \mathcal{O}_{F, v}$ and let the 'free part' of the $p$-adic neben characters vary arbitrarily. Let $\varepsilon^{\prime}=\otimes_{v} \varepsilon_{v}^{\prime}$ be a character of $T_{\mathrm{U}(1,1)}\left(\hat{\mathcal{O}}_{F}\right)$. First look at a $p$ adic place $v$. Note that $\mathcal{O}_{F, v}^{\times}=\Delta \times \Gamma$ for $\Delta \simeq \mathbb{F}_{p^{e_{v}}}^{\times}\left(e_{v}=\left[F_{v}: \mathbb{Q}_{p}\right]\right)$ and $\Gamma=$ $1+p \mathcal{O}_{F, v} . T_{\mathrm{U}(1,1)}\left(\mathcal{O}_{F_{v}}\right)=\left\{\left(\bar{a}^{-1}{ }_{a}\right) \mid a \in \mathcal{O}_{\mathcal{K}, v}^{\times}\right\}, T_{\mathrm{GL}_{2}}\left(\mathcal{O}_{F, v}\right) \simeq \mathcal{O}_{F, v}^{\times} \times \mathcal{O}_{F, v}^{\times}$. For $v \mid p$, a character $\varepsilon_{v}^{\prime}$ of $T_{\mathrm{U}(1,1)}\left(\mathcal{O}_{F, v}\right)$ can be written as $\varepsilon_{v, t o r}^{\prime} \cdot \varepsilon_{v, f r}^{\prime}$ (torsion and free part) with respect to $\Delta \times \Gamma$. Let $\psi$ be a Hecke character. We can define $\psi_{v \text {,tor }}$ and $\psi_{v, f r}$ to be characters of $\mathcal{O}_{\mathcal{K}, v}^{\times}$in the same way. Since $\varepsilon_{v, f r}^{\prime}$ and $\psi_{v, f r}$ have order powers of $p$, there are unique square roots $\varepsilon_{v, f r}^{\prime 1 / 2}$ and $\psi_{v, f r}^{1 / 2}$ of them. Now suppose that for each $v \mid p$ we have

$$
\varepsilon_{v, t o r}^{\prime}\left(\left(\begin{array}{cc}
\bar{a}^{-1} & \\
& a
\end{array}\right)\right)=\psi_{v, t o r}(a)
$$

for all $a \in \mathcal{O}_{\mathcal{K}, v}^{\times}$and that, for all $v \nmid p$,

$$
\varepsilon_{v}^{\prime}\left(\left(\begin{array}{cc}
\bar{a}^{-1} & \\
& a
\end{array}\right)\right)=\psi_{v}(a)
$$

for all $a \in \mathcal{O}_{\mathcal{K}, v}^{\times}$. Then we define a neben character of $T_{\mathrm{GL}_{2}}\left(\hat{\mathcal{O}}_{F}\right)$ by the following: for $v \nmid p$,

$$
\varepsilon_{v}\left(\left(\begin{array}{ll}
a & \\
& b
\end{array}\right)\right)=\psi_{v}(b)
$$

for $v \mid p$

$$
\varepsilon_{v, t o r}\left(\left(\begin{array}{c}
a \\
b
\end{array}\right)\right)=\psi_{v, t o r}(b)
$$

and

$$
\varepsilon_{v, f r}\left(\left(\begin{array}{c}
a \\
b
\end{array}\right)\right)=\varepsilon_{v, f r}^{1 / 2}\left(\left(\begin{array}{ll}
\frac{a}{b} & \\
& \frac{b}{a}
\end{array}\right)\right) \psi_{v, f r}^{1 / 2}(a b)
$$

and

$$
\varepsilon=\otimes_{v} \varepsilon_{v}
$$

Thus $\left.\varepsilon\right|_{T_{\mathrm{SL}_{2}\left(\hat{\mathcal{O}}_{F}\right)}}=\left.\varepsilon^{\prime}\right|_{T_{\mathrm{SL}_{2}\left(\hat{\mathcal{O}}_{F}\right)}}$.

Now let $\psi$ and $\varepsilon^{\prime}$ be as above, and let $I$ be an ideal of $\hat{\mathcal{O}}_{F}$ contained in the conductors of $\varepsilon^{\prime}$ and $\varepsilon$. Let $\varphi$ be a unitary automorphic form on $U(1,1)\left(\mathbb{A}_{F}\right)$ such that the action of $k \in U_{0}(I)$ (recall that $U_{0}(I)$ consists of matrices $\left(\begin{array}{l}a_{k} \\ c_{k}\end{array} d_{k}\right)$ such 
that $\left.c_{k} \in I\right)$ is given by $\varepsilon^{\prime}\left(\left(\begin{array}{ll}a_{k} & \\ & b_{k}\end{array}\right)\right)$. Suppose moreover that it satisfies the condition that, for any totally positive global unit $b \in \mathcal{O}_{F}^{\times}$, we have

$$
\varphi\left(\left(\begin{array}{cc}
b_{\infty}^{1 / 2} & \\
& b_{\infty}^{-1 / 2}
\end{array}\right) h_{\infty}\left(\begin{array}{cc}
\bar{a}_{j} & \\
& a_{j}^{-1}
\end{array}\right)\right) \varepsilon^{\prime}\left(\left(\begin{array}{ll}
b & \\
& 1
\end{array}\right)\right)=\varphi\left(h_{\infty}\left(\begin{array}{cc}
\bar{a}_{j} & \\
& a_{j}^{-1}
\end{array}\right)\right)
$$

for $h_{\infty} \in \mathrm{U}(1,1)\left(F_{\infty}\right)$. (This condition is necessary for a $\mathrm{SL}_{2}$ modular form to extend to a unitary Hilbert modular form on $\mathrm{GL}_{2}$ with nebentypus $\varepsilon$.)

We define a map $\alpha_{\psi}$ from $\varphi$ 's on $\mathrm{U}(1,1)\left(\mathbb{A}_{F}\right)$ as above to automorphic forms on $\mathrm{GL}_{2}\left(\mathbb{A}_{F}\right)$. Recall that by our assumption $(\triangle)$ at the beginning of this paper the Norm map from the ideal class group of $\mathcal{K}$ to that of $F$ is surjective.

Definition 64. We say that $a_{j} \bar{a}_{j} \sim g$ if $\left(\operatorname{det} g / a_{j} \bar{a}_{j}\right)$ is in the trivial narrow class group of $F$. Define

$$
\alpha_{\psi}(\varphi)(g)=\alpha_{\psi, \varepsilon, \varepsilon^{\prime}}(\varphi)(g)=\sum_{j: a_{j} \bar{a}_{j} \sim g} \varphi\left(h_{\infty}\left(\begin{array}{c}
\bar{a}_{j} \\
a_{j}^{-1}
\end{array}\right)\right) \varepsilon(k) \psi\left(z_{\infty} a_{j}\right)
$$

for $g=\gamma z_{\infty} h_{\infty}\left(\bar{a}_{j} \bar{a}_{j}\right) k \in \mathrm{GL}_{2}\left(\mathbb{A}_{F}\right)$ where $\gamma \in \mathrm{GL}_{2}(F), h_{\infty} \in \mathrm{SL}_{2}\left(F_{\infty}\right), z_{\infty} \in$ $Z\left(F_{\infty}\right), k \in \Gamma_{0}(I)_{\mathrm{GL}_{2}} \subseteq \mathrm{GL}_{2}\left(\hat{\mathcal{O}}_{F}\right)$. The (6.1) condition makes sure that this is well defined. Note also that by our assumptions on $\mathcal{K} / F$ any element $g$ can be written as the above form. This definition does not depend on the choice of $I$.

We have the following lemma.

LEMMA 65. The assumptions are as above. Suppose that $\varphi_{1}, \varphi_{3}$ are automorphic forms on $\mathrm{GU}(1,1)\left(\mathbb{A}_{F}\right)$, and that $\varphi_{2}$ is an automorphic form on $\mathrm{U}(1,1)$. Let $\psi_{1}$, $\psi_{2}, \psi_{3}$ be Hecke characters for $\mathcal{K}$. Suppose that $\psi_{1} \psi_{2} \bar{\psi}_{3}=1$ and that the central characters of $\varphi_{1}, \varphi_{3}$ are $\psi_{1}, \psi_{3}$. Suppose also that $\varepsilon_{1}^{\prime}, \varepsilon_{2}^{\prime}, \varepsilon_{3}^{\prime}$ are nebentypus of $\left.\alpha_{1}\right|_{U(1,1)}, \alpha_{2},\left.\alpha_{3}\right|_{\mathrm{U}(1,1)}$. Assume that $\varepsilon_{1}^{\prime} \varepsilon_{2}^{\prime} \bar{\varepsilon}_{3}^{\prime}=1$ and that the $\varepsilon_{i}^{\prime}$ and $\psi_{i}$ satisfy the assumptions above. Then

$$
2^{u_{\mathcal{K}}}\left[\mathcal{O}_{\mathcal{K}}^{\times}: \mathcal{O}_{F}^{\times}\right]\left\langle\varphi_{1} \varphi_{2}, \varphi_{3}\right\rangle_{U(1,1)}=\left\langle\varphi_{1} \alpha_{\psi_{2}}\left(\varphi_{2}\right), \varphi_{3}\right\rangle_{\mathrm{GL}_{2}}
$$

where $u_{\mathcal{K}}$ is some number depending only on $\mathcal{K}$. (This factor comes out when considering $\mathrm{GL}_{2} / F$ modulo the center and considering $v_{\mathcal{K}}$.)

Here we implicitly identified $\mathrm{GL}_{2}$ with a subgroup of $\mathrm{GU}(1,1)$ in the obvious way. The proof is straightforward. 


\subsection{Formulas.}

DEFINITION 66. If $f$ is a Hilbert modular form on $\mathrm{GL}_{2} / F$ with central character $\psi$, we define

$$
\begin{aligned}
f^{c}(g) & :=\overline{f\left(\left(\begin{array}{ll}
1 & \\
& -1
\end{array}\right)\right) g\left(\left(\begin{array}{ll}
1 & \\
& -1
\end{array}\right)\right)}, \\
\tilde{f}^{c} & :=f^{c} \otimes \psi(\mathrm{det}-) .
\end{aligned}
$$

\section{DEFINITION 67.}

$$
\tilde{g}_{\mathcal{D}, \beta}^{(m)}(-, x):=\left(\tilde{H}_{\mathcal{D}, \beta}^{(m)}(\alpha(x,-)) \otimes \xi(\operatorname{det}-)\right)
$$

and

$$
g_{\mathcal{D}, \beta}^{(m)}:=\operatorname{tr}_{\Gamma_{0}(M) / \Gamma_{0}\left(M_{\mathcal{D}}^{2}\right)} \pi\left(\left(\begin{array}{ll}
1 & \\
& \frac{M_{\mathcal{D}}^{2}}{M}
\end{array}\right)\right)\left(\tilde{g}_{\mathcal{D}, \beta}^{(m)}\right) .
$$

We have the following generalization of [44, Proposition 11.28]. Note that the formulas are slightly different from those in [44] since we used the adelic language. Write $a_{\mathcal{D}}(\beta, x)$ and $c_{\mathcal{D}}(\beta, x)$ for the $\beta$-Fourier coefficients for $\varphi(z, x)$ and $E(Z, x)$.

Proposition 68. The notation is as above. Let $\beta \in S_{m}(F)$.

(i) There exists a constant $C_{\mathcal{D}}^{(m)}$ depending only on $\mathcal{D}$ and $m$ such that

$$
\begin{aligned}
& \left\langle\tilde{g}_{\mathcal{D}, \beta}^{(m)}(-, x), \rho\left(\left(\begin{array}{ll}
1 & -1 \\
1 & )_{f}
\end{array}\left(\begin{array}{ll}
M_{\mathcal{D}}^{2} \prod_{v \mid p} x_{v} \bar{x}_{v} \\
\end{array}\right)_{f}\right)\left(\begin{array}{c}
1 \\
-1
\end{array}\right)_{f} \tilde{f}^{c}\right\rangle_{\Gamma_{\mathcal{D}, 0}}\right. \\
& =C_{\mathcal{D}}^{(m)} \begin{cases}a_{\mathcal{D}}(\beta, x) & m=1 \\
c_{\mathcal{D}}(\beta, x) & m=2 .\end{cases}
\end{aligned}
$$

(ii) If $\mu_{v, 1}(p) \neq 0$ for any $v \mid p$ and if $p \mid \mathfrak{f}_{\chi}$ and $p \mid \mathfrak{f}_{\chi^{-1} \xi}$ then

$$
\begin{aligned}
C_{\mathcal{D}}^{(1)}= & \left(-\pi 2^{2-\kappa} i^{-\kappa}\right)^{d} \prod_{v \nmid p, v \in \Sigma} \psi_{v}^{c} \tau_{v}\left(M_{\mathcal{D}}\right)\left|M_{\mathcal{D}}\right|_{F}^{\kappa} \gamma^{(1)}\left(\rho_{p},-z_{\kappa}\right) \\
& \times \prod_{v \mid p} \xi_{v}^{c}\left(x_{v}\right) \cdot \frac{\Gamma(\kappa-1)^{d} L_{\mathcal{K}}^{\Sigma}\left(f, \chi^{-1} \xi, \kappa-1\right)}{\Gamma(\kappa)^{d} \prod_{j=0}^{1} L^{\Sigma}\left(\chi^{-1} \xi^{\prime} \chi_{\mathcal{K}}^{j}, \kappa-j\right)} p^{r_{v}(2-\kappa / 2)},
\end{aligned}
$$


where

$$
\begin{aligned}
\gamma^{(1)}\left(\rho_{p},-z_{\kappa}\right)= & \bar{\psi}_{p}(-1) \prod_{v \mid p} c_{2}\left(\bar{\tau}_{0, v}^{\prime}, 1-\kappa / 2\right) \bar{\xi}_{v}^{c}\left(x_{v}\right) \mathfrak{g}\left(\tau_{0, v}^{\prime}\right)^{2} \cdot p^{-r_{v}+n_{v}(\kappa-2)} \\
& \times \prod_{v \mid p} \mu_{1, v}(p)^{r_{v}-n_{v}} \mathfrak{g}\left(\mu_{1, v}^{-1} \xi_{v}^{c}, x_{v}\right) \cdot \chi_{v} \bar{\xi}_{v}\left(y_{v}\right) \mathfrak{g}\left(\mu_{1, v} \bar{\chi}_{v} \xi_{v}^{c}, y_{v}\right)
\end{aligned}
$$

and $\left(y_{v}\right):=\operatorname{cond}\left(\bar{\chi}_{v} \xi_{v}^{c}\right)$ and $\left(p^{n_{v}}\right):=\left(y_{v} \bar{y}_{v}\right)$.

(iii) Suppose that we are in the generic case for any $v \mid p$. Then $C_{\mathcal{D}}^{(2)}=$ $C_{\mathcal{D}}^{(1)} \prod_{v \mid p} p^{e_{v} r_{v}}$.

Proof. One argues similarly to [44, Proposition 11.28] and the end of $[44,11.6]$.

Now we define a normalization constant

$$
\begin{aligned}
& B_{\mathcal{D}}^{(m)}:=\left|M_{\mathcal{D}}\right|_{\mathcal{K}}^{\kappa / 2} \cdot \\
& \quad\left\{\prod_{j=0}^{m}(\kappa-j-1) !^{d} \prod_{j=0}^{m} L^{\Sigma}\left(\kappa-j, \bar{\chi} \xi^{\prime} \chi_{\mathcal{K}}^{j}\right)\right. \\
& \left.\quad \times \prod_{v \in \Sigma, v \nmid p} \chi_{v} \bar{\xi}_{v}^{c}\left(y_{v} \delta_{\mathcal{K}}\right) \mathfrak{g}\left(\bar{\chi}_{v} \xi_{v}^{c}, y_{v} \delta_{\mathcal{K}}\right)\left|y_{v} \delta_{\mathcal{K}}\right|_{\mathcal{K}, v}^{2-\kappa}\right\} /\left\{\prod_{v \nmid p, v \in \Sigma} \psi_{v}^{c} \tau_{v}\left(M_{\mathcal{D}}\right) \bar{\psi}_{p}(-1)\right. \\
& \left.\quad \times \prod_{v \mid p} c_{m+1}\left(\bar{\tau}_{0, v}^{\prime},-(\kappa-m-1) / 2\right) \mathfrak{g}\left(\tau_{0, v}^{\prime}\right)^{m+1} \mathfrak{g}\left(\xi_{v}^{c}, x_{v}\right)\right\} \\
& \quad \times(-1)^{m d} 2^{m(m+1) d}(2 \pi i)^{-(m+1) d \kappa}(\pi / 2)^{m(m+2) d / 2} \\
& \quad \times\left\{\prod_{v \in \Sigma, v \nmid p} \chi_{v} \bar{\xi}_{v}^{\prime}\left(\varpi_{v}^{e_{v}}\right) q_{v}^{e_{v}(\kappa-2)} \mathfrak{g}\left(\chi_{v} \bar{\xi}_{v}^{\prime}, \varpi_{v}^{e_{v}}\right)^{-1} \quad m=2\right. \\
& \quad 11
\end{aligned}
$$

and let

$$
f_{\mathcal{D}, \beta, x}^{(m)}(-)=B_{\mathcal{D}}^{(m)} g_{\mathcal{D}, \beta}^{(m)}(-, x),
$$

where $\left(\varpi_{v}^{e_{v}}\right)$ is the conductor of $\xi_{v} \bar{\xi}_{v}^{\prime}$.

Now for $m=1$ or 2 we define

$$
L_{\mathcal{D}}^{(m)}=\frac{2^{-3 d}(2 i)^{d(\kappa+1)}}{\prod_{v \mid p} p^{e_{v} r_{v}(1-\kappa / 2)}} B_{\mathcal{D}}^{(m)} C_{\mathcal{D}}^{(m)}
$$


and

$$
S(f):=\prod_{v \mid p} \mu_{1, v}(p)^{-r^{v}} p^{e_{v} r^{v}(\kappa / 2-1)} W^{\prime}(f)^{-1},
$$

where $W^{\prime}(f)$ is the prime to $p$ part of the root number of $f$ with $\left|W^{\prime}(f)\right|_{p}=1$ (see [44, page 221]). Recall that in the subsection on notation we defined $r^{v}$ such that $p^{r^{v}} \| N_{v}$ for $v \mid p$.

Proposition 69. The assumptions are as before. Suppose that $\kappa \geqslant 2$ if $m=1$ and $\kappa>6$ if $m=2$. Suppose that $x=\operatorname{diag}\left(u,{ }^{t} \bar{u}^{-1}\right)$ with $u \in \mathrm{GL}_{m}\left(\mathbb{A}_{\mathcal{K}, f}\right)$. Suppose that $p \mid \mathfrak{f}_{\bar{\chi} \xi}$ and $p^{r} \mid \mathrm{Nm}\left(\mathfrak{f}_{\xi}\right)$. Suppose also that $\operatorname{cond}\left(\psi_{p}\right) \mid \mathfrak{f}_{\xi}^{c} \mathcal{O}_{\mathcal{K}, p}$.

$$
\begin{aligned}
& \frac{\left\langle\left(f_{\mathcal{D}, \beta, x}^{(m)} \otimes \xi\right), \rho\left(M \prod_{v \mid p}\left(p^{r_{v}}\right)_{f}\right) \tilde{f}^{c}\right\rangle_{\Gamma_{\mathcal{D}, 0}}}{\left\langle f, \rho\left(M \prod_{v \mid p}\left(p^{r_{v}}\right)\right) \tilde{f}^{c}\right\rangle_{\mathrm{GL}_{2}, \Gamma_{\mathcal{D}, 0}}} \\
& =\frac{L_{\mathcal{D}}^{(m)}}{\left.2^{-3 d}(2 i)^{d(\kappa+1)} S(f)\left\langle f, \rho\left(N^{-1}\right)\right) \tilde{f}^{c}\right\rangle_{\mathrm{GL}_{2}, \Gamma_{0}(N)}} \\
& \quad \times W^{\prime}(f)^{-1} \begin{cases}a_{\mathcal{D}}(\beta, x) & m=1 \\
c_{\mathcal{D}}(\beta, x) & m=2 .\end{cases}
\end{aligned}
$$

Here, ' $\otimes \xi$ ' means multiplying by $\xi$ odet considered as a function on $1 \times \mathrm{U}(1$, 1) $\left(\mathbb{A}_{F}\right)$ and the subscript ' $f$ ' means the finite part.

(ii)

$$
\begin{aligned}
L_{\mathcal{D}}^{(1)}= & \prod_{v \mid p} a(v, f)^{-\operatorname{ord}_{v}\left(\operatorname{Nm}\left(\mathfrak{f}_{\bar{\chi} \xi}\right)\right)}\left(\frac{(\kappa-2) !}{(-2 \pi i)^{\kappa-1}}\right)^{2 d} \\
& \times \mathfrak{g}(\bar{\chi} \xi) N m\left(\mathfrak{f}_{\bar{\chi} \xi} \delta_{\mathcal{K}}\right)^{\kappa-2} L_{\mathcal{K}}^{\Sigma}(f, \bar{\chi} \xi, \kappa-1),
\end{aligned}
$$

where $L_{\mathcal{K}}^{\Sigma}(f, \bar{\chi} \xi, \kappa-1)$ is the $\Sigma$-primitive L-function for $f$ twisted by $\bar{\chi} \xi$ over $\mathcal{K}$.

(iii) Under the hypotheses of Proposition 50(iii),

$$
L_{\mathcal{D}}^{(2)}=\prod_{v} p^{r_{v}} \times L\left(3-\kappa, \chi \bar{\xi}^{\prime}\right) \prod_{v \in \Sigma}\left(1-\bar{\chi} \xi^{\prime}\left(\varpi_{v}\right) q_{v}^{2-\kappa}\right) L_{\mathcal{D}}^{(1)} .
$$


Proof. See [44, Proposition 11.31].

COROLLARY 70. Under the hypotheses above for some choices of $x$ and $\beta$

$$
\begin{gathered}
\frac{\left\langle\left(f_{\mathcal{D}, \beta, x}^{(1)} \otimes \xi\right), \rho\left(\left(p^{r_{p}} M^{-1}\right)_{f}\right) \tilde{f}^{c}\right\rangle_{\Gamma_{\mathcal{D}, 0}}}{\left\langle f, \rho\left(\left(p^{r_{p}} M^{-1}\right)_{f}\right) \tilde{f}^{c}\right\rangle_{\mathrm{GL}_{2}, \Gamma_{\mathcal{D}, 0}}} \\
=\frac{L_{\mathcal{D}}^{(1)}}{\left.\left(2^{-3}(2 i)^{\kappa+1}\right)^{d} S(f)\left\langle f, \rho\left(N^{-1}\right)_{f}\right) \tilde{f}^{c}\right\rangle_{\mathrm{GL}_{2}, U_{0}(N)}} .
\end{gathered}
$$

Proof. See [44, Corollary 11.32]. Note that in the Hilbert modular form case the $\beta$ and $x$ are not necessarily the same as in [44]. (We need to use the assumption that $\mathrm{Nm}: C l(\mathcal{K}) \rightarrow C l(F)$ is surjective.)

For any $x \in G\left(\mathbb{A}_{F, f}\right)$ let

$$
G_{\mathcal{D}}(Z, x):=W^{\prime}(f)^{-1} L_{\mathcal{D}}^{(2)}|\mu(x)|_{F}^{-\kappa} E_{\mathcal{D}}(Z, x),
$$

and let $C_{\mathcal{D}}(\beta, x)$ be its $\beta$ th Fourier coefficient.

COROLLARY 71. Under the hypotheses as above,

$$
\begin{aligned}
& \frac{\left\langle\left(f_{\mathcal{D}, \beta, x}^{(2)} \otimes \xi\right), \rho\left(\left(p^{r_{p}} M^{-1}\right)_{f}\right) \tilde{f}^{c}\right\rangle_{\Gamma_{\mathcal{D}, 0}}}{\left\langle f, \rho\left(\left(p^{r_{p}} M^{-1}\right)_{f}\right) \tilde{f}^{c}\right\rangle_{\mathrm{GL}_{2}, \Gamma_{\mathcal{D}, 0}}} \\
& =\frac{C_{\mathcal{D}}(\beta, x)}{\left(2^{-3}(2 i)^{\kappa+1}\right)^{d} S(f)\left\langle f, \rho\left(\left(N^{-1}\right)_{f}\right) \tilde{f}^{c}\right\rangle_{\mathrm{GL}_{2}, \Gamma_{0}(N)}} .
\end{aligned}
$$

Proof. See [44, Corollary 11.33].

6.10. A formula for Fourier coefficients. Now we express certain Fourier coefficients of $G_{\mathcal{D}}(Z, x)$ as essentially Rankin-Selberg convolutions of $f$ and 
sums of theta functions. This is used later to prove various $p$-adic properties of these coefficients. Let $\mathcal{D}=(f, \psi, \xi, \Sigma)$ be an Eisenstein datum. We assume that

for any $v \mid p, \pi_{v}, \phi_{v}, \psi_{v}, \tau_{v}$ are in the generic case.

Let $\lambda$ be an idele class character of $\mathbb{A}_{\mathcal{K}}^{\times}$such that

- $\left.\lambda\right|_{\mathbb{A}_{F}^{\times}}=1$;

- $\lambda_{v}(x)=\left(x_{v} /\left|x_{v}\right|\right)^{-2}$ for all $v \mid \infty$;

- $\lambda_{v}$ is unramified if $v \nmid \Sigma \backslash\{v \mid p\}$.

Let $a_{1}, \ldots, a_{h_{\mathcal{K}}} \in \mathbb{A}_{\mathcal{K}}^{\times}$be representatives of the class group of $\mathcal{K}$ as in the previous sections such that $a_{i}=\left(\varpi_{v_{i}}, 1\right)$ for some place $v_{i}$ of $F$ splitting in $\mathcal{K}$. Also, for $i \in I_{1}$ (defined in Section 2.1), $a_{i} \bar{a}_{i}$ is trivial in the narrow class group of $F$. Let $q_{i} \in F^{\times}$be a generator. For such $i$ we assume that $\varpi_{v_{i}}=q_{i}$. Let $\mathcal{Q}=\left\{v_{i}\right\}_{i \in I_{1}}$.

Let $\beta \in S_{2}(F), \beta>0$, and $u \in \mathrm{GL}_{2}\left(\mathbb{A}_{\mathcal{K}, f}\right)$ be such that

- $u_{v} \in \mathrm{GL}_{2}\left(\mathcal{O}_{\mathcal{K}, v}\right)$ for $v \notin \mathcal{Q}$

- ${ }^{t} \bar{u}_{v} \beta u_{v} \in S_{2}\left(\mathcal{O}_{F, v}\right) *$ for all primes $v$;

- ${ }^{t} \bar{u} \beta u$ is $v$-primitive for all $v \notin \Sigma \backslash\{v \mid p\}$;

- if $u^{-1} \beta^{-1} \bar{u}^{-1}=\left(\begin{array}{l}* * \\ *\end{array}\right)$ then $d_{v} \in \mathcal{O}_{F, v}$ for all $v \in \Sigma \backslash\{v \mid p\}$.

Let $M_{\mathcal{D}}$ be as before and also satisfying

$$
\operatorname{cond}(\lambda) \mid M_{\mathcal{D}} \text { and } D_{\mathcal{K}} \operatorname{det}^{t} \bar{u} \beta u \mid M_{\mathcal{D}} \text {. }
$$

All Weil representations that show up in the following are defined using the splitting determined by the character $\lambda$. By our choice of $\mathcal{K}$, there is an idele $\mathfrak{d}_{1}$ of $\mathbb{A}_{\mathcal{K}}$ so that $\mathfrak{d}_{1} \overline{\mathfrak{d}}_{1}=\mathfrak{d}$. Later we are going to choose $u$ and $\beta$ such that they do not belong to $\mathrm{GL}_{2}\left(\mathcal{O}_{v}\right)$ only when $v=v_{i}$ for some $v_{i}$ above. Let $g^{\eta}=\eta^{-1} g \eta$ and $g^{\prime}=\left(\begin{array}{ll}{ }^{1} & -1\end{array}\right) g\left(\begin{array}{ll}{ }^{1} & \\ & -1\end{array}\right)$. Recall that we have proved the following.

For $v=v_{i} \in \mathcal{Q}$

$$
\begin{aligned}
& F J_{\beta, v}\left(f ; z_{\mathcal{K}}, x, g_{v}, u_{v} y_{v}\right) \\
& \quad=\frac{\tau\left(\operatorname{det} u_{v} y_{v}\right)\left|\operatorname{det} u_{v} y_{v}\right|_{\mathcal{K}}^{z+1 / 2}}{\prod_{j=0}^{1} L\left(2 z+3-j, \bar{\tau}_{v}^{\prime} \chi_{\mathcal{K}, v}^{j}\right)} f_{1}^{s p h}\left(g_{v}\right) \omega_{\beta}\left(u_{v}, g_{v}\right) \Phi_{0, y_{v}}(x) .
\end{aligned}
$$


For $v \in \Sigma \backslash\{v \mid p\}$ (note that we have restricted ourselves to the case when the local characters $\psi_{v}, \tau_{v}$ are unramified),

$$
\begin{aligned}
& \sum_{a \in\left(\mathcal{O}_{\mathcal{K}, v} / M_{\mathcal{D}}\right)^{\times}} F J_{\beta, v}\left(z ; x, g_{v}\left(\begin{array}{cc}
a^{-1} & \\
& \bar{a}
\end{array}\right), r_{v} u_{v}\right) \\
& =\tau\left(\operatorname{det} r_{v} y_{v}\right)\left|\operatorname{det} r_{v}\right|_{\mathcal{K}}^{-z+1 / 2}
\end{aligned}
$$

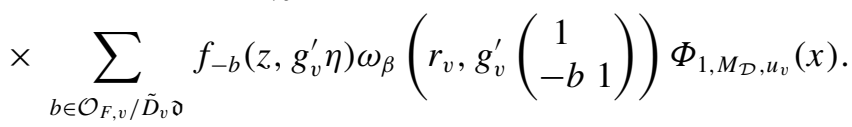

For these $v$, we have

$$
\begin{aligned}
& f_{-b, v}\left(\left(\begin{array}{cc}
1 & \\
-n & 1
\end{array}\right) g^{\prime} \eta\right)=\left(\tau_{v} \lambda_{v}^{-1}\right)(-1) f_{-b, v}\left(\left(\begin{array}{cc}
1 & \\
-n & 1
\end{array}\right) g_{v} \eta\right) \\
& =\left(\tau_{v} \lambda_{v}^{-1}\right)\left(\tilde{\delta}_{\mathcal{K}} \overline{\mathfrak{d}}_{1}\right)^{-1}\left|\delta_{\mathcal{K}} \overline{\mathfrak{d}}_{1}\right|^{\kappa / 2} f_{v}^{\dagger} \\
& \times\left(\left(\begin{array}{cc}
1 & \\
-n & 1
\end{array}\right) g_{v} \eta\left(\begin{array}{rr}
1 & b \\
& 1
\end{array}\right)\left(\begin{array}{lll}
\tilde{\delta}_{\mathcal{K}} \overline{\mathfrak{d}}_{1} & \\
& \overline{\tilde{\delta}}_{\mathcal{K}}^{-1} \overline{\mathfrak{d}}_{1}^{-1}
\end{array}\right)\right) \text {. }
\end{aligned}
$$

For $v \mid p$,

$$
\begin{aligned}
& \sum_{a \in\left(\mathcal{O}_{\mathcal{K}, v} / x_{v}\right)^{\times}} \mu_{1, v}^{-1} \xi_{v}^{c} \tau(a) F J_{\beta, v}\left(-z ; x, g_{v}\left(\begin{array}{c}
a^{-1} \\
\bar{a}
\end{array}\right), r_{v} u_{v}\right) \\
& =\psi_{v}(-1) \bar{\tau}_{v}^{\prime}(\operatorname{det} h) \mathfrak{g}\left(\tau_{v}^{\prime}\right)^{2} \bar{\tau}_{v}^{\prime}\left(p^{2 u_{v}}\right) p^{-4 u_{v} z-5 u_{v}} \tau\left(\operatorname{det} r_{v} y_{v}\right)\left|\operatorname{det} r_{v}\right|_{\mathcal{K}}^{-z+1 / 2}|\operatorname{det} h|_{v}^{2 z+1} \\
& \quad \times f_{u_{v}, 1}\left(z, g_{v} \eta\right) \omega_{\beta}\left(r_{v}, g_{v}\right) \Phi_{\xi^{c} \mu_{1, v}^{-1}, x_{v}, u_{v}}(x)
\end{aligned}
$$

(Here $x_{v}$ is a generator of the conductor of $\xi^{c} \mu_{1, v}^{-1}$. Here we use the bad notation $u_{v}$, the last one means the $v$-component of $u$ while the other $u_{v}$ means the conductor defined at the end of Section 6.7.)

For $h \in \mathrm{U}_{\beta}\left(\mathbb{A}_{F}\right), u \in \mathrm{GL}_{2}\left(\mathbb{A}_{\mathcal{K}}\right)$, we define

$$
\tilde{\Phi}:=\tilde{\Phi}_{\mathcal{D}, \beta, u}=\bigotimes_{v \mid \infty} \Phi_{\beta, v} \prod_{v \mid p} \Phi_{\beta, \xi_{v}^{c} \mu_{1, v}^{-1}, x_{v}, u_{v}} \bigotimes_{v \nmid p} \Phi_{\beta, 1, M_{\mathcal{D}}, u_{v}} \bigotimes_{v \notin \Sigma} \Phi_{0, u_{v}}
$$

and $\Phi:=\Phi_{\mathcal{D}, \beta, u}=\lambda\left(\mathfrak{d}_{1} \delta_{\mathcal{K}}^{-1}\right)^{-1}\left|\mathfrak{d}_{1} \delta_{\mathcal{K}}^{-1}\right|_{\mathcal{K}}^{-1} \omega\left(\left(\begin{array}{cc}\mathcal{d}_{1} \delta_{\mathcal{K}}^{-1} & \\ & \overline{\mathfrak{d}}_{1}^{-1} \bar{\delta}_{\mathcal{K}}\end{array}\right) \eta^{-1}\right) \tilde{\Phi}_{\mathcal{D}, \beta, u}$, and define

$$
\Theta_{\mathcal{D}, \beta}(h, g ; u):=\Theta_{\beta}\left(h, g ; \Phi_{\mathcal{D}, \beta, u}\right) .
$$


The following formula will be useful in Equation (6.4):

$$
\begin{aligned}
& \left(\omega_{\beta \mathfrak{d}^{-1}, v}\left(\eta\left(\begin{array}{rr}
1 & n \\
& 1
\end{array}\right) g_{v}^{\eta}\left(\begin{array}{rr}
1 & b \\
& 1
\end{array}\right) \eta^{-1}\right) \tilde{\Phi}\right)\left(v \mathfrak{d}_{1}\right)
\end{aligned}
$$

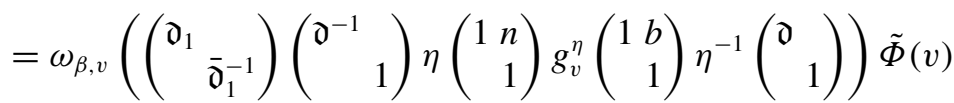

$$
\begin{aligned}
& =\lambda\left(\mathfrak{d}_{1} \delta_{\mathcal{K}}^{-1}\right)\left|\mathfrak{d}_{1} \delta_{\mathcal{K}}^{-1}\right| \mathcal{K} \omega_{\beta, v}\left(\left(\begin{array}{ll}
\overline{\mathfrak{d}}_{1}^{-1} & \\
& \overline{\mathfrak{d}}_{1}^{-1}
\end{array}\right) \eta\left(\begin{array}{rr}
1 & n \\
& 1
\end{array}\right)\right. \\
& \left.\times g_{v}^{\eta}\left(\begin{array}{rr}
1 & b \\
& 1
\end{array}\right)\left(\begin{array}{ll}
1 & \\
& \mathfrak{d}
\end{array}\right)\left(\begin{array}{cc}
\delta_{\mathcal{K}} \mathfrak{d}_{1}^{-1} & \\
& \bar{\delta}_{\mathcal{K}}^{-1} \overline{\mathfrak{d}}_{1}
\end{array}\right)\right) \Phi(v) \\
& =\lambda\left(\mathfrak{d}_{1} \delta_{\mathcal{K}}^{-1}\right)\left|\mathfrak{d}_{1} \delta_{\mathcal{K}}^{-1}\right|_{\mathcal{K}} \omega_{\beta, v}\left(\eta\left(\begin{array}{rr}
1 & n \\
& 1
\end{array}\right) g_{v}^{\eta}\left(\begin{array}{rr}
1 & b \\
& 1
\end{array}\right)\left(\begin{array}{rl}
\tilde{\delta}_{\mathcal{K}} & \\
& \overline{\tilde{\delta}}_{\mathcal{K}}^{-1}
\end{array}\right)\right) \Phi(v) .
\end{aligned}
$$

To see this, observe that

$$
\tilde{\Phi}=\lambda\left(\mathfrak{d}_{1} \delta_{\mathcal{K}}^{-1}\right)\left|\mathfrak{d}_{1} \delta_{\mathcal{K}}^{-1}\right|_{\mathcal{K}} \omega_{\beta}\left(\eta\left(\begin{array}{ll}
\delta_{\mathcal{K}} \mathfrak{d}_{1}^{-1} & \\
& \bar{\delta}_{\mathcal{K}}^{-1} \overline{\mathfrak{d}}_{1}
\end{array}\right)\right) \Phi
$$

and

$$
\omega_{\beta \mathfrak{d}^{-1}}(g)=\omega_{\beta}\left(\left(\begin{array}{cc}
\mathfrak{d}^{-1} & \\
& 1
\end{array}\right) g\left(\begin{array}{ll}
\mathfrak{d} & \\
& 1
\end{array}\right)\right) .
$$

DEFINITION 72. Let

$$
\bigotimes f_{v}=f_{\mathcal{D}}:=\bigotimes_{v \mid \infty} f_{\kappa} \bigotimes_{v \mid p} f_{u_{v}} \bigotimes_{v \in \Sigma, v \nmid p} f_{v}^{\dagger} \bigotimes_{v \notin \Sigma} f_{v}^{s p h} \in I_{1}\left(\frac{\tau}{\lambda}\right)
$$

and define $\mathcal{E}_{\mathcal{D}}$ to be the corresponding Eisenstein series on $\mathrm{U}(1,1)\left(\mathbb{A}_{F}\right)$.

We define

$$
\begin{aligned}
& C_{\mathcal{D}}(\beta, r, u) \\
& =\frac{(2 \pi i)^{2 \kappa d}(2 / \pi)^{d}\left|\delta_{\mathcal{K}}\right|_{\mathcal{K}}^{-1 / 2}\left|\delta_{F}\right|_{F}^{-1} \chi \bar{\xi}(\operatorname{det} r u)|\operatorname{det} r u|_{\mathcal{K}}^{-\kappa / 2+2} \prod_{v_{i} \mid \infty}\left(\operatorname{det} \beta_{v_{i}}^{\kappa-2}\right)}{\prod_{j=0}^{1}\left((\kappa-1-j) !^{d} L^{\Sigma}\left(\kappa-j, \bar{\chi} \xi^{\prime} \chi_{\mathcal{K}}^{j}\right)\right)} \\
& \quad \times \psi_{p}(-1) \bar{\chi}_{p} \xi_{p}^{\prime}(\operatorname{det} \beta)|\operatorname{det} \beta|_{p}^{\kappa-2} \prod_{v \mid p} \mathfrak{g}^{2}\left(\chi_{v} \bar{\xi}_{v}^{\prime}, p\right) \bar{\chi}_{p} \xi_{p}\left(p^{2 u_{p}}\right) p^{u_{p}(1-2 \kappa)}
\end{aligned}
$$

If $x=\left({ }^{h u}{ }^{t}(\overline{h u})^{-1}\right)$ for $u \in \mathrm{GL}_{2}\left(\mathbb{A}_{\mathcal{K}}\right), h \in \mathrm{U}_{\beta}\left(\mathbb{A}_{F}\right)$ satisfies the assumptions at the beginning of this subsection, then, using the useful formula before Definition 72, 
we get that if $g$ is such that $g_{f}^{\prime} \in B\left(\mathbb{A}_{F, f}\right) U_{0}\left(M_{\mathcal{D}}^{2} \tilde{D}_{\mathcal{K}} \mathfrak{d} p^{r_{p}}\right)$ we have

$$
\begin{aligned}
& \tilde{H}_{\mathcal{D}, \beta}\left(z_{k}, \alpha\left(x, g^{\prime}\right)\right) \operatorname{diag}\left(\mathfrak{d}_{1}^{-1}, \mathfrak{d}_{1}^{-1}, 1, \overline{\mathfrak{d}}_{1}, \overline{\mathfrak{d}}_{1}, 1\right) \\
& =\sum_{a \in\left(\hat{\mathcal{O}} / x_{p} M_{\mathcal{D}}\right)^{\times}} \mu_{p, 1}^{-1} \xi^{c} \tau(a) H_{\mathcal{D}, \beta} \\
& \times\left(\alpha\left(x, g^{\prime}\left(\begin{array}{cc}
a^{-1} & \\
& \bar{a}
\end{array}\right)\right) \operatorname{diag}\left(\mathfrak{d}_{1}^{-1}, \mathfrak{d}_{1}^{-1}, 1, \overline{\mathfrak{d}}_{1}, \overline{\mathfrak{d}}_{1}, 1\right)\right) \\
& =C_{\mathcal{D}}(\beta, r, u) \\
& \times \sum_{n \in F} \sum_{v \in \mathcal{K}^{2}} \sum_{b} \prod_{v} f_{-b, v}\left(\left(\begin{array}{cc}
1 & 1 \\
-n & 1
\end{array}\right) g_{v} \eta\right)
\end{aligned}
$$

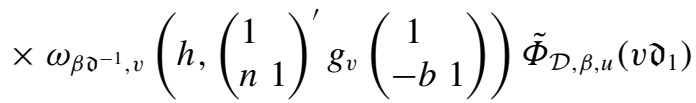

$$
\begin{aligned}
& =\left|\tilde{\delta}_{\mathcal{K}} \overline{\mathfrak{d}}_{1}\right|_{\mathcal{K}}^{\kappa / 2-1} \tau\left(\tilde{\delta}_{\mathcal{K}} \overline{\mathfrak{d}}_{1}\right)^{-1} C_{\mathcal{D}}(\beta, r, u) \\
& \times \sum_{n} \sum_{v} \sum_{b} \prod_{v} f_{v}\left(\left(\begin{array}{cc}
1 & \\
-n & 1
\end{array}\right) g_{v} \eta\left(\begin{array}{rr}
1 & b \\
& 1
\end{array}\right)\left(\begin{array}{cc}
\tilde{\delta}_{\mathcal{K}} & \\
& \overline{\tilde{\delta}}_{\mathcal{K}}^{-1}
\end{array}\right)\left(\begin{array}{cc}
\overline{\mathfrak{d}}_{1} & \\
& \mathfrak{d}_{1}^{-1}
\end{array}\right)\right) \\
& \times \omega_{\beta, v}\left(\eta\left(\begin{array}{rr}
1 & n \\
& 1
\end{array}\right) g_{v}^{\eta}\left(\begin{array}{rr}
1 & b \\
& 1
\end{array}\right)\left(\begin{array}{cc}
\tilde{\delta}_{\mathcal{K}} & \\
& \overline{\tilde{\delta}}_{\mathcal{K}}^{-1}
\end{array}\right)\right) \Phi_{\mathcal{D}, \beta, u}(v) \\
& =\left|\tilde{\delta}_{\mathcal{K}} \overline{\mathfrak{d}}_{1}\right|_{\mathcal{K}}^{\kappa / 2-1} \tau\left(\tilde{\delta}_{\mathcal{K}} \overline{\mathfrak{d}}_{1}\right)^{-1} C_{\mathcal{D}}(\beta, r, u) \\
& \times \sum_{b} \rho\left(\eta\left(\begin{array}{rr}
1 & b \\
& 1
\end{array}\right)\left(\begin{array}{cc}
\tilde{\delta}_{\mathcal{K}} & \\
& \overline{\tilde{\delta}}_{\mathcal{K}}^{-1}
\end{array}\right)\right) \Theta_{\mathcal{D}, \beta}(h, g ; u) \\
& \times \rho\left(\eta\left(\begin{array}{rr}
1 & b \\
& 1
\end{array}\right)\left(\begin{array}{cc}
\tilde{\delta}_{\mathcal{K}} & \\
& \overline{\tilde{\delta}}_{\mathcal{K}}^{-1}
\end{array}\right)\left(\begin{array}{ll}
\overline{\mathfrak{d}}_{1} & \\
& \mathfrak{d}_{1}^{-1}
\end{array}\right)\right) \mathcal{E}_{\mathcal{D}}(g) .
\end{aligned}
$$

The last step is because $\Theta_{\beta}$ is an automorphic form. Here we use $\rho$ for the right action of $\mathrm{U}(1,1)$. By strong approximation this is true for all $g$.

Now let $x=\left(\begin{array}{ccc}h u \mathrm{~d}_{1}^{-1} & & \\ & { }^{t} \bar{h}^{-1} t_{\bar{u}}^{-1} t_{\overline{\mathfrak{d}}}\end{array}\right)$. Then

$$
\begin{aligned}
& \left\langle\tilde{g}_{\mathcal{D}, \beta}^{(m)}(-, x), \rho\left(\left(\begin{array}{ll}
-1 & 1
\end{array}\right)_{f}\left(\begin{array}{ll}
1 & M_{\mathcal{D}}^{2} \prod_{v \mid p}\left(p^{r_{v}}\right)_{v}
\end{array}\right)\left({ }^{1}{ }^{-1}\right)_{f}\right) \tilde{f}^{c}\right\rangle_{\Gamma_{\mathcal{D}}, 0} \\
& =\left|\tilde{\delta}_{\mathcal{K}} \overline{\mathfrak{d}}_{1}\right|_{\mathcal{K}}^{(\kappa / 2)-1} \tau\left(\tilde{\delta}_{\mathcal{K}} \overline{\mathfrak{d}}_{1}\right)^{-1}\left\langle\sum_{b} \rho\left(\eta\left(\begin{array}{cc}
1 & b \\
1
\end{array}\right)\left(\begin{array}{c}
\tilde{\delta}_{\mathcal{K}} \\
\overline{\tilde{\delta}}_{\mathcal{K}}^{-1}
\end{array}\right)\right)\left(\Theta_{\mathcal{D}, \beta} \otimes \xi\right)(h,-, u) .\right. \\
& \rho\left(\eta\left(\begin{array}{ll}
1 & b \\
& 1
\end{array}\right)\left(\begin{array}{ccc}
\tilde{\delta}_{\mathcal{K}} \overline{\mathfrak{d}}_{1} & \\
& \tilde{\tilde{\delta}}_{\mathcal{K}}^{-1} \mathfrak{d}_{1}{ }^{-1}
\end{array}\right)\right) \mathcal{E}_{\mathcal{D}},\left.\rho\left(\left(\begin{array}{ll}
{ }^{-1} & 1
\end{array}\right)_{f}\left(\begin{array}{ll}
1 & \\
& M_{\mathcal{D}}^{2} \prod_{v \mid p}\left(p^{r_{v}}\right)_{v}
\end{array}\right)\left(\begin{array}{ll}
{ }_{1} & -1
\end{array}\right)_{f}\right) \tilde{f}^{c}\right|_{\Gamma_{\mathcal{D}, 0}}
\end{aligned}
$$




$$
\begin{aligned}
& =\left|\tilde{\delta}_{\mathcal{K}} \overline{\mathfrak{d}}_{1}\right|_{\mathcal{K}}^{(\kappa / 2)-1} \tau\left(\tilde{\delta}_{\mathcal{K}} \overline{\mathfrak{d}}_{1}\right)^{-1}\left\langle\sum_{b} \rho\left(\left(\begin{array}{cc}
1 & b \\
& 1
\end{array}\right)\left(\begin{array}{cc}
\tilde{\delta}_{\mathcal{K}} & \\
& \overline{\tilde{\delta}}_{\mathcal{K}}^{-1}
\end{array}\right)\right)\left(\Theta_{\mathcal{D}, \beta} \otimes \xi\right)(h,-; u) .\right.
\end{aligned}
$$

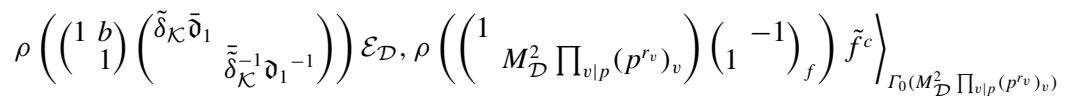

$$
\begin{aligned}
& =\left|\tilde{\delta}_{\mathcal{K}} \overline{\mathfrak{d}}_{1}\right|_{\mathcal{K}}^{(\kappa / 2)-1} \tau\left(\tilde{\delta}_{\mathcal{K}} \overline{\mathfrak{d}}_{1}\right)^{-1}\left\langle\rho\left(\left(\begin{array}{cc}
\mathfrak{d}_{1}^{-1} & \\
& \overline{\mathfrak{d}}_{1}
\end{array}\right)\right) A_{\beta}^{\prime}(h,-; u) \cdot \mathcal{E}_{\mathcal{D}},\right. \\
& \left.\rho\left(\left(\begin{array}{cc}
\tilde{\delta}_{\mathcal{K}}^{-1} \overline{\mathfrak{d}}_{1}^{-1} & \\
& \overline{\tilde{\delta}}_{\mathcal{K}} \mathfrak{d}_{1}
\end{array}\right)\left(\begin{array}{ll}
1 & \\
& M_{\mathcal{D}}^{2} \prod_{v \mid p}\left(p^{r_{v}}\right)_{v}
\end{array}\right)\left(\begin{array}{ll}
1 & -1
\end{array}\right)_{f}\right) \tilde{f}^{c}\right\rangle_{\Gamma_{0}\left(M_{\mathcal{D}}^{2} \tilde{D}_{\mathcal{K}} \mathfrak{d} \prod_{v \mid p} p^{r_{v}}\right)} \\
& =\left|\tilde{\delta}_{\mathcal{K}} \overline{\mathfrak{d}}_{1}\right|_{\mathcal{K}}^{\kappa / 2-1} \xi\left(\tilde{\delta}_{\mathcal{K}} \overline{\mathfrak{d}}_{1}\right)\left\langle\rho\left(\left(\begin{array}{ll}
\mathfrak{d}_{1}^{-1} & \\
& \overline{\mathfrak{d}}_{1}
\end{array}\right)\right) A_{\beta}^{\prime}(h,-; u) \cdot \mathcal{E}_{\mathcal{D}},\right. \\
& \left.\rho\left(\left(\begin{array}{cc}
1 & \\
& \tilde{D}_{\mathcal{K} \mathfrak{d}}
\end{array}\right)\left(\begin{array}{cc}
1 & \\
& M_{\mathcal{D}}^{2} \prod_{v \mid p}\left(p^{r_{v}}\right)_{v}
\end{array}\right)\left(\begin{array}{ll}
1^{1} & -1
\end{array}\right)_{f}\right) \tilde{f}^{c}\right|_{\Gamma_{0}\left(M_{\mathcal{D}}^{2} \tilde{D}_{\mathcal{K}} \mathfrak{d} \prod_{v \mid p} p^{r_{v}}\right)}
\end{aligned}
$$

where $\left.A_{\beta}^{\prime}=\left(\Theta_{\mathcal{D}, \beta} \otimes \xi\right)(h,-; u)\right)$. Now, for $v_{i}$ with $i \in I_{1}$, by definition we have that $v_{i} \bar{v}_{i}$ is an ideal of $F$ generated by a totally positive global element. Let us take such a generator $q_{i}$. Also we take representatives $\left\{b_{j}\right\}_{j}$ of the coset:

$\left\{b\right.$ : totally positive units in $\left.\mathcal{O}_{F}^{\times}\right\} /\left\{c \bar{c}\right.$ for $c$ a unit in $\left.\mathcal{O}_{\mathcal{K}}^{\times}\right\}$.

Then we define

$$
A^{\prime}(g):=\sum_{i, j, k} \Theta_{\mathcal{D}, \beta_{i j k}}(h, g, u) . \xi(\operatorname{det} g)
$$

where $\beta_{i j k}=\left(\begin{array}{cc}b_{j} & \\ & q_{i} b_{k}\end{array}\right)$.

REMARK 73. The reason for introducing such $b_{j}$ is to make sure that the $A^{\prime}$ satisfy $(*)$ in the subsection for nebentypus (by checking the $q$-expansion) and can be extended later to some theta functions on $\mathrm{GL}_{2}$.

Definition 74. Let $\alpha_{\xi \lambda}$ be the operator $\alpha$ defined in Definition 64 using the character $\xi \lambda$. We define

$$
A:=\alpha_{\xi \lambda} A^{\prime}
$$

(Recall that $A^{\prime}$ is a form in $\mathrm{U}(1,1)$ and $A$ is a form on $\mathrm{GL}_{2}\left(\mathbb{A}_{F}\right)$.)

These $A$ and $A^{\prime}$ will be used in Proposition 92 in Section 8 . We are in a position to state our formula for the Fourier coefficients for Klingen Eisenstein series. Before this let us do some normalizations:

$$
B_{\mathcal{D}, 1}=\frac{(\kappa-3) !^{d} L^{\Sigma}\left(\kappa-2, \bar{\chi} \xi^{\prime}\right)}{(-2)^{d}(2 \pi i)^{(\kappa-2) d} \prod_{v \mid p}\left(\mathfrak{g}\left(\chi_{v} \bar{\xi}_{v}^{\prime}, p\right)\right) \bar{\chi}_{v} \xi_{v}^{\prime}\left(p^{u_{p}}\right) p^{(2-\kappa) u_{p}}},
$$




$$
:=\frac{\left|M_{\mathcal{D}}\right|_{\mathcal{K}}^{\kappa / 2} 2^{3 d_{i}}{ }^{-2 d}\left|\delta_{\mathcal{K}}\right|_{\mathcal{K}}^{-1 / 2}\left|\delta_{F}\right|_{F}^{-1} \prod_{v \mid \Sigma \backslash\{v \mid p\}} \chi_{v} \bar{\xi}_{v}^{c}\left(y_{v} \delta_{\mathcal{K}}\right) \mathfrak{g}\left(\bar{\chi}_{v} \xi_{v}^{c}, y_{v} \delta_{\mathcal{K}}\right)\left|y_{v} \delta_{\mathcal{K}}\right|_{\mathcal{K}, v}^{2-\kappa}}{\mathfrak{g}\left(\xi_{p}^{c}, x_{p}\right)}
$$

$$
B_{\mathcal{D}}(\beta, r, u):=\frac{\psi \bar{\xi}(\operatorname{det} r u)|\operatorname{det} r u|_{\mathcal{K}}^{(\kappa / 2)+2} \bar{\chi}_{p} \xi_{p}^{\prime}(\operatorname{det} \beta)|\operatorname{det} \beta|_{p}^{\kappa-2} \prod_{v_{i} \mid \infty}\left(\operatorname{det} \beta_{v_{i}}^{\kappa-2}\right)}{\prod_{v \mid \Sigma /\{v \mid p\}} \bar{\chi}_{v} \xi_{v}^{\prime}\left(\varpi_{v}^{e_{v}}\right) q_{v}^{e_{v}(2-\kappa)} \mathfrak{g}\left(\chi_{v} \bar{\xi}_{v}^{\prime}\right)} .
$$

Then

$$
B_{\mathcal{D}}^{(2)} C_{\mathcal{D}}(\beta, r, u)=B_{\mathcal{D}}(\beta, r, u) B_{\mathcal{D}, 1} B_{\mathcal{D}, 2}
$$

The following proposition follows immediately from Proposition 68 and the previous calculation.

PROPOSITION 75. With the assumptions at the beginning of this subsection, let

$$
\beta \in S_{2}(F), \quad \beta \gg 0,
$$

and $u, h, x$ be as before. Then

$$
\begin{aligned}
& \frac{C_{\mathcal{D}}(\beta, x)}{2^{-3 d}(2 i)^{(\kappa-1) d} S(f)\left\langle f, \rho\left(\left(_{N}^{-1}\right)\right) \tilde{f}^{c}\right\rangle_{\mathrm{GL}_{2}, \Gamma_{0}(N)}} \\
& =\left|\tilde{\delta} \mathcal{K} \overline{\mathfrak{d}}_{1}\right|_{\mathcal{K}}^{(\kappa / 2)-1} \xi\left(\tilde{\delta} \mathcal{K} \overline{\mathfrak{d}}_{1}\right) B_{\mathcal{D}}(\beta, h, u) B_{\mathcal{D}, 1} \mathcal{E}_{\mathcal{D}}(-) B_{\mathcal{D}, 2} \\
& \frac{\left\langle\pi\left(\left(\begin{array}{cc}
\mathfrak{d}_{1}^{-1} & \\
& \overline{\mathfrak{d}}_{1}
\end{array}\right)\right) A_{\beta}^{\prime}(h,-; u), \rho\left(\left(M_{\mathcal{D}}^{2} \tilde{D}_{\mathcal{K}^{\mathfrak{d}}}{ }^{-1}\right)\left(\prod_{v \mid p} p^{r_{v}}{ }^{-1}\right)\right) \tilde{f}^{c}\right\rangle_{\Gamma_{0}\left(\prod_{v \mid p} p^{r_{v}} M_{\mathcal{D}}^{2} \tilde{D}_{\mathcal{K} \mathfrak{d})}\right.} .}{\left\langle f, \rho\left(\left(\prod_{v \mid p} p^{r_{v}}{ }^{-1}\right)\left(M^{-1}\right)\right) \tilde{f}^{c}\right\rangle_{\mathrm{GL}_{2}, \Gamma_{\mathcal{D}}}} .
\end{aligned}
$$

Now let us make some choices for the $u$ and $\beta$ and record some formulas for the theta kernel functions. We remark that our convention for $\Phi$ is slightly different

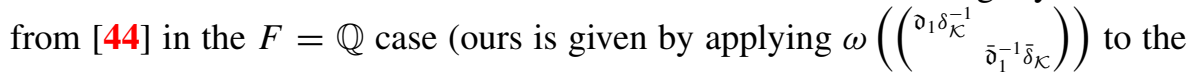
one chosen by [44]).

Let $\gamma_{0} \in \mathrm{GL}_{2}\left(\mathbf{A}_{\mathcal{K}, f}\right)$ be such that $\gamma_{0, v}=(\eta, 1)$ for $v \mid p$ and $\gamma_{0, v}=1$ otherwise. We let $u_{i}=\gamma_{0}\left(\begin{array}{c}1 \\ a_{i}^{-1}\end{array}\right)$. Then the $\beta_{i j k}, u_{i}$ satisfy the assumptions at the beginning of this subsection. 
The following formulas follow from our computations in Section 5. For $v \mid p$,

$$
\begin{aligned}
& \Phi_{\beta_{i j k}, \xi_{v}^{c} \mu_{1, v}^{-1}, x_{v}, \gamma_{0, p}}(x)= \\
& \begin{cases}\bar{\xi}_{v, 2} \mu_{1, v}^{-1}\left(b_{k} q_{i} x_{2}^{\prime \prime}\right) g\left(\xi_{v, 2}\right) \bar{\xi}_{v, 1} \mu_{1, v}^{-1}\left(x_{1}^{\prime} b_{j}\right) g\left(\xi_{v, 1}\right) & x_{1}=\left(x_{1}^{\prime}, x_{2}^{\prime \prime}\right) \in \mathcal{O}_{F, v}^{\times} \times \mathcal{O}_{F, v} \\
0 & x_{2}=\left(x_{2}^{\prime}, x_{2}^{\prime \prime}\right) \in \mathcal{O}_{F, v}^{\times} \times \mathcal{O}_{F, v} \\
0 & \text { otherwise. }\end{cases}
\end{aligned}
$$

For $j \in I$, then

$$
\omega_{\beta_{i j k}}\left(g_{\infty_{j}}\right) \Phi_{\beta_{i j k}, \infty_{j}}(x)=e\left(\mathrm{Nm}\left(x_{1}\right) b_{j} w\right) e\left(\mathrm{Nm}\left(x_{2}\right) b_{k} q_{i} w\right) j\left(g_{\infty_{j}}, i\right)^{-2} .
$$

Also, if $v \nmid p, v \in \Sigma$,

$$
\Phi_{\beta_{i j k}, 1, M_{\mathcal{D}}, 1}(x)=\left|D_{v}\right|^{-1} \lambda_{v}(-1)\left|M_{\mathcal{D}}^{2}\right|_{v}^{-1} \begin{cases}1-\frac{1}{q_{v}^{\prime}}, & x_{1} \in M_{\mathcal{D}} \mathcal{O}_{v}, x_{2} \in \mathcal{O}_{v}, \\ -\frac{1}{q_{v}^{\prime}}, & x_{2} \in \mathcal{O}_{v}, x_{1} \in \frac{M_{\mathcal{D}}}{\varpi_{v}} \mathcal{O}_{v}^{\times}, \\ 0 & \text { otherwise }\end{cases}
$$

for $v$ nonsplit, and

$$
\begin{aligned}
& \Phi_{\beta_{i j k}, 1, M_{\mathcal{D}, 1}}(x)=\left|D_{v}\right|^{-1} \lambda_{v}(-1)\left|M_{\mathcal{D}}\right|_{v}^{-1} \\
& \begin{cases}\left(1-\frac{1}{q_{v}}\right)^{2}, & x_{1} \in \frac{M_{\mathcal{D}} \mathcal{O}_{v}}{\mathfrak{d}_{1}}, x_{2} \in \frac{\mathcal{O}_{v}}{\mathfrak{d}_{1}}, \\
-\frac{1}{q_{v}}\left(1-\frac{1}{q_{v}}\right), & x_{2} \in \frac{\mathcal{O}_{v}}{\mathfrak{d}_{1}}, x_{1} \in\left(\frac{M_{\mathcal{D}}}{\varpi_{v^{\prime}}} \mathcal{O}_{F_{v}}^{\times} \times \frac{\mathcal{O}_{F_{v}}^{\times}}{\mathfrak{d}_{1}}\right) \\
& \text { or }\left(\frac{\mathcal{O}_{F_{v}}^{\times}}{\mathfrak{d}_{1}} \times \frac{M_{\mathcal{D}}}{\varpi_{v^{\prime}} \mathcal{d}_{1}} \mathcal{O}_{F_{v}}^{\times}\right), \\
\frac{1}{q_{v}^{2}}, & x_{2} \in \frac{\mathcal{O}_{v}}{\mathfrak{d}_{1}}, x_{1} \in \frac{M_{\mathcal{D}}}{\varpi_{v} \mathfrak{d}_{1}} \mathcal{O}_{v}^{\times} \times \frac{M_{\mathcal{D}}}{\varpi_{v} \mathfrak{d}_{1}} \mathcal{O}_{v}^{\times}, \\
0 & \text { otherwise, }\end{cases}
\end{aligned}
$$

for $v$ split.

If $v=v_{i}$,

$$
\Phi_{0, u_{i}, v}(x)= \begin{cases}1 & x_{1} \in \mathcal{O}_{\mathcal{K}, v}, x_{2} \in a_{i}^{-1} \mathcal{O}_{\mathcal{K}, v} \\ 0 & \text { otherwise }\end{cases}
$$


6.11. Identify with Rankin-Selberg convolutions. From now on we assume that all characters $(\chi, \xi, \tau)$ are unramified outside $p$. We recall the notion of Rankin-Selberg convolution for Hilbert modular forms, following [15]. Let $f$ and $g$ be two Hilbert automorphic forms (as functions on $\mathrm{GL}_{2}\left(\mathbb{A}_{F}\right)$ ) with level dividing some $\mathfrak{m}$. For simplicity, we assume that both $f$ and $g$ have unitary central characters $\chi$ and $\xi$ and have parallel weight $k$ and $\kappa$ such that $k>\kappa$. Let $\tau=\chi / \xi$ and $\Gamma_{\infty}=N\left(\mathcal{O}_{F}\right)$ define Eisenstein series

$$
\mathscr{E}(x ; s)=\sum_{\gamma \in \Gamma_{\infty} \backslash \Gamma_{0}(\mathfrak{m})} \tau(\gamma x) \eta(\gamma x)^{s} j\left(\gamma, x_{\infty}\left(z_{0}\right)\right)^{k-\kappa}\left|j\left(\gamma, x_{\infty}\left(z_{0}\right)\right)\right|^{\kappa-k}
$$

as in [15, page $341(4.5)$ ], where $\eta$ is defined at the bottom of [15, page 341]. Suppose that the nebentypus of $g$ and $f$ differ by the nebentypus of $\mathscr{E}$ (this satisfies $[15,4.5])$. Consider the following integral:

$$
Z(s, f, g, \tau)=\int_{F_{\mathbb{A}_{+}}^{\times} / F_{+}} \int_{F_{\mathbb{A}} / F} \Phi\left(f^{c}, g\right)\left(\left(\begin{array}{ll}
y & x \\
0 & 1
\end{array}\right)\right) \tau(y)|y|_{\mathbb{A}}^{s} d x d^{\times} y,
$$

where $\Phi\left(f^{c}, g\right)(x)=\overline{f^{u}(x)} g^{u}(x)\left|j\left(x_{\infty}, z_{0}\right)^{-k-\kappa}\right|, f^{u}(x)=D^{-1} f(x) j\left(x_{\infty}, z_{0}\right)^{k}$, and $g^{u}(x)=D^{-1} g(x) j\left(x_{\infty}, z_{0}\right)^{\kappa}$, with $D$ the discriminant of $F / \mathbb{Q}$. Note that there are minor differences between the notation here and in [15], and that the $m$ and $\mu$ there are 0 in our case. Now for any ideal $\mathfrak{a} \subset \mathcal{O}_{F}$ we define a value $a\left(\mathfrak{a}, f^{c}\right) a(\mathfrak{a}, g) \tau(\mathfrak{a})$ as follows. Take any $t \in \mathbb{A}_{F}^{\times}$which represents the ideal $\mathfrak{a}$, and define

$$
a\left(\mathfrak{a}, f^{c}\right) a(\mathfrak{a}, g) \tau(\mathfrak{a})=a\left(t, f^{c}\right) a(t, g) \tau(t)
$$

if $\mathfrak{a}$ is prime to the conductor or $\tau$ and is 0 otherwise. This definition does not depend on the choice of $t$ by our assumption on nebentypus. Then

$$
Z(s, f, g, \tau)=D^{(1+2 s) / 2} \tau(\mathfrak{d})^{-1}(4 \pi)^{-d(s+(k+\kappa) / 2)} \Gamma(s+k / 2+\kappa / 2)^{d} D(s, f, g),
$$

where

$$
D(s, f, g)=\sum_{\mathcal{O}_{F} \supset \mathfrak{a} \neq 0} a\left(\mathfrak{a}, f^{c}\right) a(\mathfrak{a}, g) \tau(\mathfrak{a}) \mathrm{Nm}_{F / \mathbb{Q}}(\mathfrak{a})^{-s} .
$$

By (4.7) in [15], up to a nonzero constant

$$
\begin{aligned}
Z(s, f, g, \tau)= & D^{-2} \int_{X_{0}} \bar{f} g(x) \\
& \times \mathscr{E}(x ; s+1) j\left(x_{\infty}, z_{0}\right)^{\kappa-k}\left|j\left(x_{\infty}, z_{0}\right)^{k-\kappa}\right| d x,
\end{aligned}
$$

where $X_{0}:=\mathrm{GL}_{2}(F) \backslash \mathrm{GL}_{2}\left(\mathbb{A}_{F}\right) / U_{0}(\mathfrak{m}) \mathbb{A}_{F}^{\times} C_{\infty}^{+}$. Note that our formula is a special case of (4.7) in [15], and is easier due to our assumptions on the nebentypus. 
Let $\alpha_{f}=\left(a_{v}\right)_{v} \in \mathrm{GL}_{2}\left(\mathbb{A}_{F, f}\right)$ be defined by $a_{v}=\left({ }_{M_{\mathcal{D}}^{2} \tilde{D}_{\mathcal{K}} \mathfrak{d}}{ }^{-1}\right)$ of $v \in \Sigma \backslash\{p\}$ and $a_{v}=1$ otherwise. For $m \geqslant 0$, let $b_{m} \in \mathrm{GL}_{2}\left(\mathbb{A}_{F, f}\right)$ be defined by $b_{m, v}=\left({ }_{p^{m}}{ }^{-1}\right)$ for $v \mid p$ and $b_{m, v}=1$ for $v \nmid p$. Then

$$
\rho(\alpha) \mathcal{E}_{\mathcal{D}}=E\left(f_{\mathcal{D}}^{\prime}, z_{\kappa} ; \gamma_{\infty}\right),
$$

where $f_{\mathcal{D}}^{\prime}(z, g):=f_{\mathcal{D}}\left(z, g \alpha_{f}^{-1}\right) \in I_{1}(\tau / \lambda)$. It follows that $f_{\mathcal{D}}^{\prime}(z, g)$ is supported on

$$
B_{1}\left(\mathbb{A}_{F}\right) \eta K_{1, \infty}^{+} N_{B_{1}}\left(\hat{\mathcal{O}}_{F}\right) \alpha=B_{1}\left(\mathbb{A}_{F}\right) K_{1, \infty}^{+} K_{1}\left(p^{u_{p}} M_{\mathcal{D}}^{2} \tilde{D}_{\mathcal{K}} \mathfrak{d}\right)
$$

and that for $g=b k_{\infty} k_{f}$ in the support we have $f_{\mathcal{D}}^{\prime}(z, g)=\left|M_{\mathcal{D}}^{2} \tilde{D}_{\mathcal{K}} \mathfrak{d}\right|_{F}^{-(\kappa / 2-1)} \tau \bar{\lambda}\left(d_{b} d_{k_{f}}\right)\left|a_{b} / d_{b}\right|_{\mathbb{A}_{F}}^{z+1 / 2} J_{1}\left(k_{\infty}, i\right)^{2-\kappa}, \quad b=\left(\begin{array}{rr}a_{b} & * \\ & d_{b}\end{array}\right)$.

Suppose that $h \in S_{2}\left(p^{r_{p}} M_{\mathcal{D}}^{2} \tilde{D}_{\mathcal{K}}\right)$ is a normalized eigenform such that the nebentypus of $\mathcal{E}_{\mathcal{D}} \cdot h$ is the same as $f$. Then

$$
\begin{aligned}
& B_{\mathcal{D}, 1}\left\langle\mathcal { E } _ { \mathcal { D } } \rho \left(\left(\begin{array}{cc} 
& 1 \\
-1 & )_{v \nmid p, \infty}
\end{array}\left(\begin{array}{lll}
\tilde{D}_{\mathcal{K}} \mathfrak{d} M_{\mathcal{D}}^{2} & \\
& & 1
\end{array}\right)_{f}\right) h, \rho\right.\right. \\
& \times\left(\left.\left(\begin{array}{cc}
1 \\
-1
\end{array}\right)_{f}\left(\begin{array}{ll}
\tilde{D}_{\mathcal{K}} \mathfrak{d} M_{\mathcal{D}}^{2} \prod_{v \mid p} p_{v}^{r_{v}} & \\
&
\end{array}\right) \tilde{f}_{f}\right|_{\Gamma_{\mathcal{D}, 0}}\right.
\end{aligned}
$$

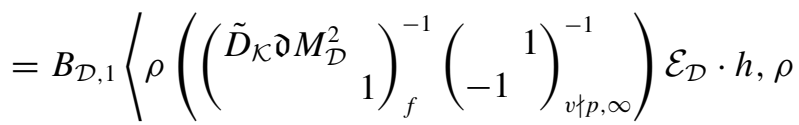

$$
\begin{aligned}
& \times\left(\left(\begin{array}{c}
\tilde{D}_{\mathcal{K}} \mathfrak{d} M_{\mathcal{D}}^{2} \prod_{v \mid p} p_{v}^{r_{v}} \\
\end{array}\right)_{f}^{-1}\left(\begin{array}{c}
1 \\
-1
\end{array}\right)_{v \nmid p, \infty}^{-1}\right.
\end{aligned}
$$

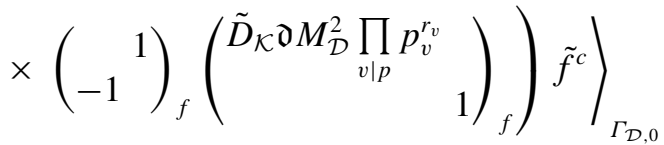

$$
\begin{aligned}
& =B_{\mathcal{D}, 1}\left\langle\rho \left(( \begin{array} { l l } 
{ \tilde { D } _ { \mathcal { K } } \mathfrak { d } M _ { \mathcal { D } } ^ { 2 } } & { } \\
{ } & { 1 }
\end{array} ) _ { f } \left(\begin{array}{c}
1 \\
\\
\end{array}\right.\right.\right.
\end{aligned}
$$

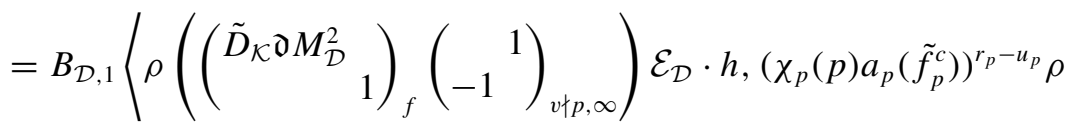

$$
\begin{aligned}
& \left.\times\left(\left(\prod_{v \mid p} p_{v}^{u_{v}}\right)_{p}\right) \tilde{f}^{c}\right\rangle
\end{aligned}
$$




$$
\begin{aligned}
= & D^{(\kappa+1) / 2} \tau(\mathfrak{d})^{-1}\left|M_{\mathcal{D}}^{2} \tilde{D}_{\mathcal{K}}\right|_{F}^{\kappa / 2-1}\left(\bar{\chi}_{p}(p) a\left(f_{p}\right)\right)^{r_{p}-u_{p}} B_{\mathcal{D}, 1}(4 \pi)^{(1-\kappa) d} \Gamma(\kappa-1)^{d} \\
& \times D\left(\rho\left(\left(\begin{array}{c}
-1 \\
p^{u_{p}}
\end{array}\right){ }_{p}\right) \tilde{f}^{c, u}, h^{u} ; \kappa-1\right) \\
= & D^{(\kappa+1) / 2} \tau(\mathfrak{d})^{-1}\left|M_{\mathcal{D}}^{2} \tilde{D}_{\mathcal{K}}\right|_{F}^{\kappa / 2-1}\left(\bar{\chi}_{p}(p) a_{p}\left(f_{p}\right)\right)^{r_{p}-u_{p}} \\
& \times B_{\mathcal{D}, 1} \overline{c(f)}\left(\xi_{p}(p) a_{p}\left(h_{p}\right)\right)^{u_{p}-r}(4 \pi)^{(1-k) d} \Gamma(\kappa-1)^{d} \\
& \times L^{\Sigma}\left(\kappa-2, \bar{\chi} \xi^{\prime}\right)^{-1} L\left(f_{1}^{c} \times h, \kappa-1\right) .
\end{aligned}
$$

Here the superscript $u$ means the unitarization. We get the following.

LEMMA 76. The assumptions are as above. Suppose that $h \in S_{2}\left(p^{r_{p}} M_{\mathcal{D}}^{2} \tilde{D}_{\mathcal{K}}\right)$ is a normalized eigenform on $\mathrm{GL}_{2}\left(\mathbb{A}_{F}\right)$. Then

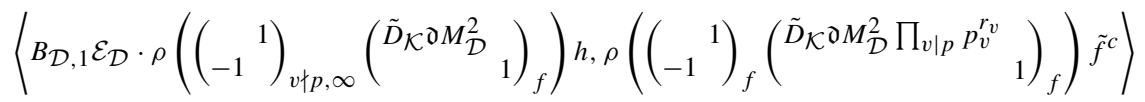

$$
\begin{aligned}
& =B_{\mathcal{D}, 3} L\left(\tilde{f}^{c} \times h, \kappa-1\right),
\end{aligned}
$$

where

$$
\begin{aligned}
B_{\mathcal{D}, 3}:= & \left|M_{\mathcal{D}}^{2} \tilde{D}_{\mathcal{K}}\right|_{F}^{\kappa / 2-1}\left(\bar{\chi}_{p}(p) a_{p}\left(f_{p}\right)\right)^{r_{p}-u_{p}} \\
& \times B_{\mathcal{D}, 1} \overline{c(f)}\left(\xi_{p}(p) a_{p}\left(h_{p}\right)\right)^{u_{p}-r}(4 \pi)^{(1-\kappa) d} \Gamma(\kappa-1)^{d} \times L^{\Sigma}\left(\kappa-2, \bar{\chi} \xi^{\prime}\right)^{-1}
\end{aligned}
$$

\section{7. $p$-adic interpolations}

7.1. $p$-adic Eisenstein datum. As in [44, Section 12], we define the $p$-adic Eisenstein datum to be $\mathbf{D}=(A, \mathbb{I}, \mathbf{f}, \psi, \Sigma)$ which consists of:

- The integer ring $A$ of a finite extension of $\mathbb{Q}_{p}$.

- II, a local finite integral domain over $\Lambda_{W, A}$.

- A nearly ordinary $\mathbb{I}$-adic normalized eigenform $\mathbf{f}$ which is new at all $v \nmid p$ such that some specialization $f_{\kappa_{0}}$ is of parallel weight $\kappa_{0}$ and trivial character.

- A finite-order Hecke character $\psi$ of $\mathbb{A}_{\mathcal{K}}^{\times} / \mathcal{K}^{\times}$and (cond $\left.\psi\right) \mid p$ and $\left.\psi\right|_{\mathbb{A}_{F}^{\times}}=1$.

- A finite-order Hecke character $\xi$ of $\mathcal{K}$ such that (cond $\xi) \mid p$, and the $p$-adic avatar of $\xi \cdot|\cdot|^{\kappa_{0} / 2-1}$ factors through $\Gamma_{\mathcal{K}}$.

- A finite set $\Sigma$ of primes containing all primes dividing $N \delta_{\mathcal{K}}$ and conductors of $\psi$ and $\xi$.

REMARK 77. For simplicity we have assumed that $\psi$ is unramified outside $p$ and that the $\chi_{\mathbf{f}}$ in $[44,12.1]$ is trivial. The assumption on the $p$-adic avatar is used to 
require that the central critical value $L\left(f_{\kappa_{0}}, \kappa_{0} / 2\right)$ is an interpolation point of our family so that we can apply Theorem 86.

Recall also that we have defined in Section 3.1 the maps $\alpha$ and $\beta$. Let $\boldsymbol{\psi}:=$ $\alpha \circ \omega \psi \Psi_{\mathcal{K}}^{-1}$ and $\xi:=\left(\beta \circ \Psi_{\mathcal{K}}\right) \cdot \xi$.

DEFINITION 78. Recall that we define $\mathcal{X}_{\mathrm{D}}^{a}$ to be the set of arithmetic weights $\phi$ such that $\kappa_{\phi} \geqslant 6$. Let $\mathcal{X}_{\mathbf{D}}^{\text {gen }} \subset \mathcal{X}_{\mathbf{D}}^{a}$ be the subset such that the local Eisenstein datum is generic as defined in Section 4.1.3.

For $\phi \in \mathcal{X}_{\mathbf{D}}^{a}$ we define

$$
\psi_{\phi}(x):=\left(\prod_{\sigma \in \Sigma} x_{\sigma}^{-\kappa_{\phi}} x_{v_{\sigma}}^{\kappa_{\phi}}\right)(\phi \circ \psi(x))|x|_{\mathcal{K}}^{\kappa_{\phi} / 2} .
$$

Here $v_{\sigma}$ is the $p$-adic place corresponding to $\sigma$ under $\iota: \mathbb{C} \simeq \mathbb{C}_{p}$. We also define

$$
\xi_{\phi}:=\phi \circ \xi
$$

\subsection{Interpolation.}

7.2.1. Congruence module and the canonical period. Suppose that $R$ is a finite extension of $\mathbb{Z}_{p}$. We let $\mathbb{T}_{\kappa}^{\text {ord }}\left(M p^{r}, \varepsilon ; R\right)\left(\mathbb{T}_{\kappa}^{0, \text { ord }}\left(M p^{r}, \varepsilon ; R\right)\right)$ be the $R$-subalgebra of $\operatorname{End}_{R}\left(M_{\kappa}^{\text {ord }}\left(M p^{r}, \varepsilon ; R\right)\right.$ ) (respectively, $\operatorname{End}_{R}\left(S_{\kappa}^{\text {ord }}\left(M p^{r}, \varepsilon ; R\right)\right)$ ) generated by the Hecke operators $T_{v}$ (these are Hecke operators defined using the double coset

$$
U_{1}(N)_{v}\left(\begin{array}{cc}
\varpi_{v} & \\
& 1
\end{array}\right) U_{1}(N)_{v}
$$

for the $v)$. For any $f \in S_{\kappa}^{\text {ord }}(N, \varepsilon ; R)$ a nearly ordinary eigenform we have

$$
1_{f} \in \mathbb{T}_{\kappa}^{0, \text { ord }}(N, \varepsilon ; R) \otimes_{R} F_{R}=\mathbb{T}_{\kappa}^{\prime} \times F_{R}
$$

as the projection onto the second factor. Let $\mathfrak{m}_{f}$ be the maximal ideal of $\mathbb{T}_{\kappa}^{\text {ord }}(N$, $\varepsilon ; R$ ) corresponding to $f$.

Suppose that the localization of the Hecke algebra at $\mathfrak{m}_{f}$ satisfies the Gorenstein property. Then $\mathbb{T}^{\text {ord, } 0}(M, \varepsilon ; R)_{\mathfrak{m}_{f}}$ is a Gorenstein $R$-algebra, so $\mathbb{T}^{\text {ord, } 0}(M, \varepsilon ; R) \cap$ $\left(0 \oplus F_{R}\right)$ is a rank-one $R$-module. We let $\ell_{f}$ be a generator; so $\ell_{f}=\eta_{f} 1_{f}$ for some $\eta_{f} \in R$.

Suppose that $\mathbf{f} \in M^{\text {ord }}(M, \mathbb{I})$ is a nearly ordinary $\mathbb{I}$-adic cuspidal eigenform. Then, as above,

$$
\mathbb{T}^{\text {ord }, 0}(M, \mathbb{I}) \otimes F_{\mathbb{I}} \simeq \mathbb{T}^{\prime} \times F_{\mathbb{I}},
$$


where $F_{\mathbb{I}}$ is the fraction field of $\mathbb{I}$ and projection onto the second factor gives the eigenvalues for the actions on $\mathbf{f}$. Again let $1_{\mathbf{f}}$ be the idempotent corresponding to projection onto the second factor. Then for an $\mathbf{g} \in S^{\text {ord }}(M, \mathbb{I}) \otimes_{\mathbb{I}} F_{\mathbb{I}}, 1_{\mathbf{f}} \mathbf{g}=c \mathbf{f}$ for some $c \in F_{\mathbb{I}}$. As above, under the Gorenstein property for $\mathbf{f}$, we can define $\ell_{\mathbf{f}}$ and $\eta_{\mathbf{f}}$.

DEFINITION 79. For a classical point $f_{\phi}$ of $\mathbf{f}$ under the Gorenstein assumption the canonical period of $f_{\phi}$ is defined by

$$
\Omega_{f_{\phi}, \text { can }}:=\frac{2^{-3 d}(2 i)^{\left(\kappa_{\phi}+1\right) d} S_{f_{\phi}}\left\langle f_{\phi}, \rho\left(\left(N^{-1}\right)_{f}\right) \tilde{f}_{\phi}^{c}\right\rangle_{\Gamma_{0}\left(N_{\phi}\right)}}{\eta_{f_{\phi}}} .
$$

(The $N_{\phi}$ is the conductor of $\mathbf{f}_{\phi}$.)

REMARK 80. This 'canonical' period depends on the generator $\ell_{\mathbf{f}}$.

For $\mathcal{X} \subseteq \mathcal{X}_{\mathbf{D}}^{a}$ a Zariski dense set of points we define $\mathcal{M}_{\mathcal{X}}\left(M, \Lambda_{\mathbf{D}}\right)$ to be the space of (finite set of) formal $q$ expansions which when specializing to $\phi \in \mathcal{X}$ is a classical modular form with nebentypus $\varepsilon_{\phi}$. The [44, Lemma 12.2] is true as well for the Hilbert modular forms (the character $\theta$ there is assumed to be trivial in our situation).

LEMMA 81. There exists an idempotent $e \in \operatorname{End}_{\Lambda_{\mathbf{D}}}\left(\mathcal{M}_{\mathcal{X}}\left(M ; \Lambda_{\mathbf{D}}\right)\right)$ such that, for any $\mathbf{g} \in \mathcal{M}_{\mathcal{X}}\left(M ; \Lambda_{\mathbf{D}}\right),(e \mathbf{g})_{\phi}=e \mathbf{g}_{\phi} \in M_{\kappa_{\phi}}^{\text {ord }}\left(M p^{t_{\phi}}, \varepsilon_{\phi} ; \phi\left(\Lambda_{\mathbf{D}}\right)\right)$ for all $\phi \in \mathcal{X}^{a}$, and such that $\mathcal{M}_{\mathcal{X}}\left(M ; \Lambda_{\mathbf{D}}\right)=\mathcal{M}_{\mathcal{X}}(M ; \mathbb{I}) \otimes_{\mathbb{I}} \Lambda_{\mathbf{D}}$.

We also have an analog of [44, Lemma 12.2.4, 12.2.5] (the key interpolation lemma) in the Hilbert modular case and the proofs are completely the same. We omit the details. They are used in constructing the $p$-adic $L$-functions and $p$-adic Eisenstein series in the next two subsections.

7.3. $\quad \boldsymbol{p}$-adic $\boldsymbol{L}$-functions. Now we state the main theorem for the existence of the nonintegral and integral $p$-adic $L$-functions following $[44,12.3]$.

THEOREM 82. Let $A, \mathbb{I}, \mathbf{f}, \xi$, and $\Sigma$ be as before. Suppose that there exists a finite $A$-valued idele class character $\psi$ of $\mathbb{A}_{\mathcal{K}}^{\times}$such that $\left.\psi\right|_{\mathbb{A}_{F}^{\times}}=\chi_{\mathbf{f}}$ and $\psi$ is unramified outside $\Sigma$. 
(i) There exists $\tilde{\mathcal{L}}_{\mathbf{f}, \mathcal{K}, \xi}^{\Sigma} \in F_{\mathbb{I}} \otimes_{\mathbb{I}} \mathbb{I}_{\mathcal{K}}$ such that, for any $\phi \in \mathcal{X}_{\mathbf{D}}^{\text {gen }}, \tilde{\mathcal{L}}_{\mathbf{f}, \mathcal{K}, \xi}^{\Sigma} \in F_{\mathbb{I}} \otimes_{\mathbb{I}} \mathbb{I}_{\mathcal{K}}$ is finite at $\phi$ and

$$
\begin{aligned}
\phi\left(\tilde{\mathcal{L}}_{\mathbf{f}, \mathcal{K}, \xi}^{\Sigma}\right) & =\prod_{v \mid p} \mu_{1, v, \phi}(p)^{-\operatorname{ord}_{v}\left(\operatorname{Nm}\left(\mathfrak{f}_{\bar{\chi}_{\boldsymbol{f}_{\phi}} \xi_{\phi}}\right)\right)} \\
\times & \frac{\left(\left(\kappa_{\phi}-2\right) !\right)^{2 d} \mathfrak{g}\left(\bar{\chi}_{\mathbf{f}_{\phi}} \xi_{\phi}\right) \operatorname{Nm}\left(\mathfrak{f}_{\bar{\chi}_{\mathbf{f}_{\phi}} \xi_{\phi}} \delta_{\mathcal{K}}\right)^{\kappa_{\phi}-2} L_{\mathcal{K}}^{\Sigma}\left(\mathbf{f}_{\phi}, \bar{\chi}_{\mathbf{f}_{\phi}} \xi_{\phi}, \kappa_{\phi}-1\right)}{(-2 \pi i)^{2 d\left(\kappa_{\phi}-1\right)} 2^{-3 d}(2 i)^{d\left(\kappa_{\phi}+1\right)} S\left(\mathbf{f}_{\phi}\right)\left\langle\mathbf{f}_{\phi}, \rho\left(\left(N^{-1}\right)_{f}\right) \tilde{\mathbf{f}}_{\phi}^{c}\right\rangle_{\Gamma_{0}(N)}} .
\end{aligned}
$$

(ii) Suppose that the localization of the Hecke algebra at $\mathfrak{m}_{f}$ is Gorenstein. Then there exists $\mathcal{L}_{\mathbf{f}, \mathcal{K}, \xi}^{\Sigma} \in \mathbb{I}_{\mathcal{K}}$ such that, for any $\phi \in \mathcal{X}_{\mathbf{D}}^{\text {gen }}, \mathcal{L}_{\mathbf{f}, \mathcal{K}, \xi}^{\Sigma}$ is finite at $\phi$ and

$$
\begin{aligned}
\phi\left(\mathcal{L}_{\mathbf{f}, \mathcal{K}, \xi}^{\Sigma}\right)= & \prod_{v \mid p} \mu_{1, v, \phi}(p)^{-\operatorname{ord}\left(\operatorname{Nm}\left(\mathfrak{f}_{\bar{\chi}_{f_{\phi}} \xi_{\phi}}\right)\right)} \\
& \times \frac{\left(\left(\kappa_{\phi}-2\right) !\right)^{2 d} \mathfrak{g}\left(\bar{\chi}_{\mathbf{f}_{\phi}} \xi_{\phi}\right) N m\left(\mathfrak{f}_{\bar{\chi}_{\mathfrak{x}_{\phi}} \xi_{\phi}} \delta_{\mathcal{K}}\right)^{\kappa_{\phi}-2} L_{\mathcal{K}}^{\Sigma}\left(\mathbf{f}_{\phi}, \bar{\chi}_{\mathbf{f}_{\phi}} \xi_{\phi}, \kappa_{\phi}-1\right)}{(-2 \pi i)^{2 d\left(\kappa_{\phi}-1\right)} \Omega_{f_{\phi}, c a n}} .
\end{aligned}
$$

Recall that in Remark 12 the $\mu_{1, v}$ are defined as follows: the $v$ component $\pi_{v}$ of $\pi_{f_{\phi}}$ is such that $\pi_{v} \simeq \pi\left(\mu_{1, v}, \mu_{2, v}\right)$ and $\mu_{1, v}(p)$ has $p$-adic valuation $-\left(\kappa_{\phi}-1\right) / 2$ and $\mu_{2, v}(p)$ has $p$-adic valuation $\left(\kappa_{\phi}-1\right) / 2$. In terms of the $q$-expansion (2.1), if $y_{v} \in \mathbb{A}_{F}^{\times}$is the element which is $p$ at $v$ and is 1 at other places, then

$$
\mu_{1, v}(p)=a\left(y_{v} \operatorname{diag}\left(\mathfrak{d}^{-1}, 1\right), f_{\phi}\right) \cdot p^{-\left(\kappa_{\phi}-1\right) / 2} .
$$

Proof. The proof is similar to that of [44, Proposition 12.6]. We first interpolate the Fourier coefficients of the $f_{\mathcal{D}, \beta}(-, x)$ defined in (6.2) (get a $\Lambda_{\mathbf{D}}$-adic expansion) in the same way as in [44, Proposition 12.3]. Now there arises a difference from the situation in [44]: these are only forms on $U(1,1)$ instead of $\mathrm{GL}_{2} / F$. So we consider

$$
\sum_{j} f_{\mathcal{D}, \beta}^{(1)}\left(\left(\begin{array}{cc}
b_{j, \infty}^{1 / 2} & \\
& b_{j, \infty}^{-1 / 2}
\end{array}\right) g\right) \varepsilon^{-1}\left(\left(\begin{array}{ll}
b_{j} & \\
& 1
\end{array}\right)_{f}\right)
$$

instead (as functions of $g$ ), where the $b_{j}$ are defined before Remark 73 and $\varepsilon$ is the neben character for the $f_{\mathcal{D}, \beta}(-, x)$. By checking the $q$-expansions we see equation (7.1) satisfies (6.1). So it makes sense to define

$$
\alpha_{\psi}\left(\sum_{j} f_{\mathcal{D}, \beta}^{(1)}\left(\left(\begin{array}{cc}
b_{j, \infty}^{1 / 2} & \\
& b_{j, \infty}^{-1 / 2}
\end{array}\right) g\right) \varepsilon^{-1}\left(\left(\begin{array}{ll}
b_{j} & \\
& 1
\end{array}\right)_{f}\right)\right)
$$


(see Definition 64). We thus obtain an element $f_{\mathbf{D}, \beta}(-, x) \in \mathcal{M}_{\mathcal{X}}\left(M ; \Lambda_{\mathbf{D}}\right)$ in the sense of Lemma 81. Let $\beta$ and $x$ be as in Corollary 70. Then the first Fourier coefficient of $1_{f} e f_{\mathbf{D}, \beta}(-, x)$ or $\ell_{f} e f_{\mathbf{D}, \beta}(-, x)$ at $\operatorname{diag}\left(\mathfrak{d}^{-1}, 1\right)$ gives the $\tilde{\mathcal{L}}_{\mathbf{f}, \mathcal{K}, \xi}^{\Sigma}$ or $\mathcal{L}_{\mathbf{f}, \mathcal{K}, \xi}^{\Sigma}$ by Corollary 70.

We will often write $\tilde{\mathcal{L}}_{\mathbf{D}}^{\Sigma}$ and $\mathcal{L}_{\mathbf{D}}^{\Sigma}$ for $\tilde{\mathcal{L}}_{\mathbf{f}, \mathcal{K}, \xi}^{\Sigma}$ and $\mathcal{L}_{\mathbf{f}, \mathcal{K}, \xi}^{\Sigma}$

REMARK 83. In [15, Theorem I] Hida also constructed a full-dimensional $p$ adic $L$-function $\mathcal{L}_{\mathbf{f}, \mathcal{K}, \xi}^{\text {Hida }}$ for Hilbert modular Hida families (the $\mathbf{g}$ there is taken to be the Hida family of $\mathrm{CM}$ forms corresponding to characters of $\Gamma_{\mathcal{K}}$ times $\xi$ ). Our interpolation points are not quite the same as his. In fact he used differential operators to allow nonparallel weights to get the whole family while we instead allowed more general nebentypus at $p$. (Recall that Hida used the Rankin-Selberg method and required the difference of the $p$-parts of the nebentypus of $\mathbf{f}$ and $\mathbf{g}$ to come from a global character.) Hida is able to interpolate more general critical values. In particular, the point $\phi_{0}$ corresponding to the special value $L\left(f_{2}, 1\right)$ where $f_{2}$ is the element in $\mathbf{f}$ with parallel weight 2 and trivial nebentypus is an interpolation point. We look at the subfamily which is the Zariski closure $W^{\prime}$ of the arithmetic points where the specializations of both $\mathbf{f}$ and $\mathbf{g}$ are of parallel weights and trivial characters. ( $W^{\prime}$ is the spectrum of a quotient of $\mathbb{I}\left[\left[\Gamma_{\mathcal{K}}\right]\right]$ with dimension 4.) The comparison between our construction and Hida's will be useful in proving Theorem 102 since our interpolation formula did not include the central critical value $L\left(f_{\kappa_{0}}, \kappa_{0} / 2\right)$. So we state the following proposition.

Proposition 84. Up to a factor which is a unit in $\mathbb{I}\left[\left[\Gamma_{\mathcal{K}}\right]\right]$, we have

$$
\left.\mathcal{L}_{\mathbf{f}, \mathcal{K}, \xi}^{\text {Hida }}\right|_{W^{\prime}}=\left.\left(\tilde{\mathcal{L}}_{\mathbf{D}}^{\Sigma} \cdot \prod_{v \in \Sigma \backslash\{v \mid p\}} E_{\mathbf{f}, \mathcal{K}, v}^{\text {Hida }}\right)\right|_{W^{\prime}},
$$

where $\left.\right|_{W^{\prime}}$ means restriction to $W^{\prime}$, and $E_{\mathbf{f}, \mathcal{K}, \phi, v}^{H i d a}$ is the local Euler factor at $v$. They are by definition $p$-adic analytic functions since $v \nmid p$.

Proof. The proposition is proved by comparing the interpolation formula for our $\tilde{\mathcal{L}}_{\mathbf{D}}^{\Sigma}$ and the one in [15, Theorem I]. The factor mentioned in the proposition which is a unit in $\mathbb{I}\left[\left[\Gamma_{\mathcal{K}}\right]\right]$ is a product of local root numbers at primes outside $p$ for $\mathbf{f}$ and $\mathrm{CM}$ forms (it is well known that they are units). We simply point out a couple of items for comparison.

- In Hida's interpolation formula in his Theorem I there are three $p$-adic Gauss sum terms, while only two show up in our formula. The one in the denominator 
of [15] is caused by the difference in the Petersson inner product. It is well known that the ratio is indeed given by root numbers, for example, by using the global functional equation.

- Hida only proved the interpolation formula of for critical values of the RankinSelberg product of some $f$ and $g$ both having weight at least two, while in our case the corresponding $g$ is of weight one. However it is easily seen that Hida's interpolation extends to our situation as well.

We also have the $\Sigma$ primitive $p$-adic $L$-functions $\tilde{L}_{f, \mathcal{K}, \xi}^{\Sigma}$ and $L_{f, \mathcal{K}, \xi}^{\Sigma}$ for a single $f$ by specializing the one for $\mathbf{f}$ to $f$ (see [44, Theorem 12.7]).

7.3.1. Connections with anticyclotomic p-adic L-functions. Let $\beta: \Lambda_{\mathcal{K}, A} \rightarrow$ $\Lambda_{\mathcal{K}, A}^{-}$be the homomorphism which corresponds to specializing to anticyclotomic twists of the central critical values $L\left(f_{\kappa_{0}}, \kappa_{0} / 2\right)$. (The $\beta$ means this only in this subsection.) For $A$ the integer ring of a finite extension of $\mathbb{Q}_{p}, \beta$ extends to

$$
F_{A} \otimes_{A} \Lambda_{\mathcal{K}, A} \rightarrow F_{A} \otimes_{A} \Lambda_{\mathcal{K}, A}^{-},
$$

for $F_{A}$ the ring of fractions of $A$. Now we define the anticyclotomic $p$-adic $L$ function:

$$
\mathcal{L}_{f, \mathcal{K}, \xi}^{\Sigma,-}:=\beta\left(\mathcal{L}_{f, \mathcal{K}, \xi}^{\Sigma}\right) \in \Lambda_{\mathcal{K}, A}^{-}
$$

and

$$
\tilde{\mathcal{L}}_{f, \mathcal{K}, \xi, v}^{\Sigma,-}:=\beta\left(\tilde{\mathcal{L}}_{f, \mathcal{K}, \xi}^{\Sigma}\right) \in \Lambda_{\mathcal{K}, A}^{-} \otimes_{A} F_{A} .
$$

For $v \mid p$ we can further specialize $\gamma_{v^{\prime}, i}^{-}=1$ for all $v^{\prime} \neq v$ to get $\mathcal{L}_{f, \mathcal{K}, \xi, v}^{\Sigma,-}$ and $\tilde{\mathcal{L}}_{f, \mathcal{K}, \xi, v}^{\Sigma,-}$

Definition 85. For some $v \mid p, \tilde{\mathcal{L}}_{f, \mathcal{K}, \xi, v}^{\Sigma,-} \in A\left[\left[\Gamma_{v}^{-}\right]\right] \times_{\mathbb{Z}_{p}} \mathbb{Q}_{p}$, and $\mathcal{L}_{f, \mathcal{K}, \xi}^{\Sigma,-} \in A\left[\left[\Gamma_{v}^{-}\right]\right]$ when we have the Gorenstein property required to construct it. Then we say for $v, f$ satisfies the following ((NV1) or (NV2)) if:

(NV1) $\tilde{\mathcal{L}}_{f, \mathcal{K}, \xi, v}^{\Sigma,-}$ is not identically 0 ;

(NV2) $\mathcal{L}_{f, \mathcal{K}, \xi}^{\Sigma,-}$ is not divisible by $\varpi_{A}$ in $A\left[\left[\Gamma_{v}^{-}\right]\right]$where $\varpi_{A}$ is a uniformizer of $A$.

Recall that $f_{\kappa_{0}}$ is an ordinary form in the family $\mathbf{f}$ of parallel weight $\kappa_{0} \geqslant 2$ and trivial nebentypus. Write $\phi_{0} \in \mathbb{I}\left[\left[\Gamma_{\mathcal{K}}\right]\right]$ for a point corresponding to the special 
$L$-value $L\left(f_{\kappa_{0}}, \kappa_{0} / 2\right)$. Now we state a theorem giving a sufficient condition for (NV1) and (NV2). We assume the branch character $\xi=1$.

Now we state the following theorem of Hung [26].

THEOREM 86. Assume that $p \geqslant 5$. Suppose that the level of $f_{\kappa_{0}}$ is $M=M^{+} M^{-}$, where $M^{+}$and $M^{-}$are products of split and inert primes, respectively. Suppose that

(1) $M^{-}$is square free with the number of prime factors being congruent to $d$ modulo 2;

(2) $\bar{\rho}_{f}$ is ramified at all $v \mid M^{-}$.

Then for any $v \mid p(N V 1)$ is true. Suppose moreover that

(3) the assumptions in Theorem 8 are satisfied.

Then for any $v \mid p(N V 2)$ is true.

Proof. This is essentially that of [26, Theorem B] except that we need to compare the periods. We need to prove that the period defined in (5.2) of [26] is Hida's canonical period under our assumptions. We first claim that in his definition for $\left\langle f_{\pi^{\prime}}, f_{\pi^{\prime}}\right\rangle$ in Section 4.7 there, the cardinality of the stabilizer is a $p$-adic unit. Otherwise there will be a global element $\gamma \in B^{\times}(F)$ of order $p$ which means $F(\gamma)$ is a field quadratic over $F$ containing the $p$ th roots of unity. However since $p$ is unramified in $F$ the only possibility is $p=3$, which contradicts our assumption. This proves the claim, and thus this inner product is a perfect pairing. Then assumptions (1) and (2) above ensure that the local Hecke algebras corresponding to $f$ on $B$ and on $\mathrm{GL}_{2}$ are the same. Assumption (3) implies that the self-inner product $\left\langle f_{\pi^{\prime}}, f_{\pi^{\prime}}\right\rangle$ on $B$ generates the congruence module. To see this we need to know that the space of integral modular forms on $B$ of level $M$ is free of rank one over $\mathbb{T}_{\mathfrak{m}_{f}}$. This can be proved using the argument of [9, Theorem 11.2]. There is a small difference between our situation and Fujiwara's that, under the assumptions, the quaternion algebra Fujiwara considers is split at primes dividing $M^{-}$while our $B$ is ramified at those primes. But by our assumption (2) above this does not make any difference for the argument. These altogether tell us that the period in [9] is nothing but Hida's canonical period.

Now we consider our $\mathcal{L}_{\mathbf{f}, \mathcal{K}, 1}^{\Sigma}$ for $\mathbf{f}$ a parallel Hida family of eigenforms. The local Euler factors at $\Sigma \backslash\{v \mid p\}$ for split primes $v$ do not affect the anticyclotomic $\mu$-invariant (see $[44,12.3 .2]$ ). However the Euler factors at nonsplit primes are nonzero elements of $\mathbb{I}\left[\left[\Gamma^{+}\right]\right]$. We still have the following. 
LEMMA 87. Let $P$ be a height-one prime of $\mathcal{L}_{\mathbf{f}, \mathcal{K}, 1}^{\Sigma}$ which is a pullback of a heightone prime of $\mathbb{I}\left[\left[\Gamma^{+}\right]\right]$. Then

$$
\operatorname{ord}_{P} \mathcal{L}_{\mathbf{f}, \mathcal{K}, 1}^{\Sigma} \leqslant \operatorname{ord}_{P}\left(\prod_{\text {nonsplit } v \in \Sigma \backslash\{v \mid p\}} E_{\mathbf{f}, \mathcal{K}, 1, v}\right) .
$$

(Recall that $E_{\mathbf{f}, \mathcal{K}, 1, v}$ means the local Euler factor at $v$.)

Proof. The proof follows from our Remark 83 and Theorem 86.

7.4. $p$-adic Eisenstein series. We state some theorems which are straight generalizations of [44, Section 12.4].

THEOREM 88. We use the assumptions as in Theorem 82(ii). Let $\mathbf{D}=(A, \mathbb{I}$, $\mathbf{f}, \psi, \xi, \Sigma)$ be a p-adic Eisenstein datum. Suppose that (irred) and (dist) hold and that the local Hecke algebra $\mathbb{T}_{\mathfrak{m}_{\mathfrak{f}}}$ is Gorenstein. Then for each $x=\operatorname{diag}(u$, $\left.{ }^{t} \bar{u}^{-1}\right) \in G\left(\mathbb{A}_{F, f}^{\Sigma}\right)$ there exists a formal q-expansion

$$
\mathbf{E}_{\mathbf{D}}(x):=\sum_{\beta \in S(F), \beta \geqslant 0} c_{\mathbf{D}}(\beta, x) q^{\beta}, \quad c_{\mathbf{D}}(\beta, z) \in \Lambda_{\mathbf{D}},
$$

with the property that, for each $\phi \in \mathcal{X}_{\mathcal{D}}^{\text {gen }}$,

$$
\mathbf{E}_{\mathcal{D}_{\phi}}(x):=\sum_{\beta \in S(F), \beta \geqslant 0} \phi\left(c_{\mathbf{D}}(\beta, x)\right) e(\operatorname{Tr} \beta Z)
$$

is the $q$ expansion at $x$ for $G_{\mathcal{D}_{\phi}} / \Omega_{f_{\phi}, \text { can }}$. Moreover, if $\operatorname{det} \beta=0$ then $c_{\mathbf{D}}(\beta, x) \in$ $\mathcal{L}_{\mathbf{f}, \mathcal{K}, \xi}^{\Sigma} \mathcal{L}_{\xi^{\prime}}^{\Sigma} \Lambda_{\mathbf{D}}$

REMARK 89. There is also a $\tilde{\mathbf{E}}_{\mathbf{D}}$ version of the above theorem under the hypothesis of Theorem 82(i) using $1_{\mathbf{f}}$ instead of $\ell_{\mathbf{f}}$. We omit it here. Also, from the definition of $1_{\mathbf{f}}$ and the fact that the congruence number of $f_{\phi_{0}}$ is finite, we can find an element $\mathbf{b} \in \mathbb{I}$ such that $\mathbf{b} 1_{\mathbf{f}}$ is a Hecke operator with integral (I-coefficients) and such that $\mathbf{b}\left(\phi_{0}\right) \neq 0$. Now we explain how to see this using [14, Theorem II]. The Hida families are just components of the Hecke algebra $\mathbf{h}$ acting on the space of cuspidal $\Lambda_{W}$-adic nearly ordinary forms (recall that the $\Lambda_{W}$ is defined to be a complete power series ring whose coefficient ring may be assumed to contain all Fourier coefficients of $f_{\phi_{0}}$ ). We need to know that $\mathbf{f}$ is the only component passing through $\phi_{0}$. We invert $p$ and look at the map of local rings

$$
\left(\Lambda_{W} \otimes_{\mathbb{Z}_{p}} \mathbb{Q}_{p}\right)_{P_{\kappa_{\phi_{0}}}} \rightarrow\left(\mathbf{h} \otimes_{\mathbb{Z}_{p}} \mathbb{Q}_{p}\right)_{\left(\phi_{0}\right)}
$$


where the subscripts $P_{\kappa_{\phi_{0}}}$ and $\left(\phi_{0}\right)$ mean localizations at these primes ( $P_{\kappa_{\phi_{0}}}$ means the prime corresponding to the weight of $\phi_{0}$ ). We denote this as $A \rightarrow B$. Using the fact that the congruence number at $\phi_{0}$ is finite and Hida's theorem mentioned above we know that $A / \mathfrak{m}_{A} \simeq B / \mathfrak{m}_{B}$ (both isomorphic to $\left(\Lambda_{W} / P_{\kappa_{\phi_{0}}}\right) \otimes_{\mathbb{Z}_{p}} \mathbb{Q}_{p}$ since the coefficient ring of $\Lambda_{W}$ contains all Fourier coefficients of $f_{\phi_{0}}$ ) and $\mathfrak{m}_{B}=\mathfrak{m}_{A} B$. So $A \simeq B$ by Nakayama's lemma. So there are no more components passing through $\phi_{0}$ since otherwise the local ring $\left(\mathbf{h} \otimes_{\mathbb{Z}_{p}} \mathbb{Q}_{p}\right)_{\left(\phi_{0}\right)}$ cannot be isomorphic to $\left(\Lambda_{W} \otimes_{\mathbb{Z}_{p}} \mathbb{Q}_{p}\right)_{P_{\kappa_{\phi_{0}}}}$.

\section{8. $\quad p$-adic properties of Fourier coefficients of $E_{\mathrm{D}}$}

In this section, following [44, Section 13], using the theta correspondence between different unitary groups, we prove that certain Fourier coefficient of $E_{\mathbf{D}}$ is not divisible by certain height-one prime $P$.

\subsection{Automorphic forms on some definite unitary groups.}

8.1.1. Generalities. Let $\beta \in S_{2}(F), \beta \gg 0$. Let $H_{\beta}$ be the unitary group of the pairing determined by $\beta$. We write $H$ for $H_{\beta}$ sometimes for simplicity.

For an open compact subgroup $U \subseteq H\left(\mathbb{A}_{F, f}\right)$ and any $\mathbb{Z}$-algebra $R$ we let

$$
\begin{aligned}
& \mathcal{A}(U, R) \\
& \quad:=\left\{f: H\left(\mathbb{A}_{F}\right) \rightarrow R: f(\gamma h k u)=f(h), \gamma \in H(F), k \in H\left(F_{\infty}\right), u \in U\right\}
\end{aligned}
$$

and for any subgroup $K \subseteq\left(\mathbb{A}_{F, f}\right)$ let

$$
\mathcal{A}_{H}(K ; R):=\underset{U \supseteq \lim _{U}}{\longrightarrow} \mathcal{A}_{H}(U ; R) .
$$

8.1.2. Hecke operators. For a prime $v$ let $U, U^{\prime} \subset H\left(F_{v}\right)$ be open compact subgroups, and let $h \in H\left(F_{v}\right)$. We can define Hecke operators [UhU $]: \mathcal{A}(U$, $R) \rightarrow \mathcal{A}_{H}\left(U^{\prime} ; R\right)$ in the usual way.

We will be mainly interested in two cases.

Split case. Suppose that $v$ splits in $\mathcal{K}$. The identification $\mathrm{GL}_{2}\left(\mathcal{K}_{v}\right)=\mathrm{GL}_{2}\left(F_{v}\right) \times$ $\mathrm{GL}_{2}\left(F_{v}\right)$ yields an identification of $H\left(F_{v}\right)$ with $\mathrm{GL}_{2}\left(F_{v}\right)$ via projection on the first factor: $H\left(F_{v}\right)=\left\{\left(A, \beta^{-1 t} A^{-1} \beta\right) \in \mathrm{GL}_{2}\left(\mathcal{K}_{v}\right)\right\}$. We let $H_{v} \subset H\left(F_{v}\right)$ be the subgroup identified with $\mathrm{GL}_{2}\left(\hat{\mathcal{O}}_{F, v}\right)$. For $U=H_{v}$ we define $T_{v}^{H}$ as the Hecke operator $\left[U h_{v} U\right], h_{v}:=\left(\begin{array}{cc}\varpi_{v} & 1\end{array}\right) \in \mathrm{GL}_{2}\left(F_{v}\right)=H\left(F_{v}\right)$, where $\varpi_{v}$ is a uniformizer at $v$. 
Primes dividing $p$. If $v \mid p$ then $v$ splits in $\mathcal{K}$. For a positive integer $n$ we let $I_{n, v} \subset H_{v}$ be the subgroup identified with the set of $g \in \mathrm{GL}_{2}\left(\mathbb{Z}_{p}\right)$ such that $g \bmod p^{n}$ belongs to $N_{B}\left(\mathbb{Z} / p^{n} \mathbb{Z}_{p}\right)$. For $U=I_{n, v}$, we write $U_{v}^{H}$ for the Hecke operator $\left[U h_{v} U\right]$ where $h_{v}:=\left(\begin{array}{l}p_{1} \\ { }_{1}\end{array}\right)$. This operator respects variation in $n$ and commutes with the $T_{v}^{H}$ for $v \nmid p$. Let $U_{p}:=\prod_{v \mid p} U_{v}$.

Now we define the nearly ordinary projector. First recall that we have fixed an $\iota: \mathbb{C}_{p} \simeq \mathbb{C}$. Let $R$ be either a $p$-adic ring or of the form $R=R_{0} \otimes_{\mathbb{Z}_{p}} \mathbb{Q}_{p}$ with $R_{0}$ a $p$-adic ring. Then we define

$$
e_{H}:=\underset{m}{\lim } U_{p}^{H, m !} \in \operatorname{End}_{R}\left(\mathcal{A}_{H}(U ; R)\right) .
$$

It is well known that this exists and is an idempotent. We can define the Hecke operators for automorphic forms on $\mathrm{U}(1,1)$ in the split and $p$-adic case in the same way as above (using the projection onto the first factor).

\subsection{Applications to Fourier coefficients.}

8.2.1. Forms on $H \times \mathrm{U}_{1}$. If $v$ splits in $\mathcal{K}$ we always fix once for all a prime $w$ above $v$ such that $v=w \bar{w}$. Then we view representations of $H\left(F_{v}\right)$ via the respective identifications of these groups with $\mathrm{GL}_{2}\left(F_{v}\right)$ (projection onto the first factor of $\left.\mathrm{GL}_{2}\left(\mathcal{K}_{v}\right)=\mathrm{GL}_{2}\left(F_{v}\right) \times \mathrm{GL}_{2}\left(F_{v}\right)\right)$. Let $\lambda$ be a character of $\mathbb{A}_{\mathcal{K}}^{\times} / \mathcal{K}^{\times}$such that $\lambda_{\infty}(z)=(z /|z|)^{-2}$ and $\left.\lambda\right|_{\mathbb{A}_{F}^{\times}}=1$. Let $(\pi, \mathcal{V}), \mathcal{V} \subseteq \mathcal{A}_{H}$, be an irreducible representation of $H\left(\mathbb{A}_{F, f}\right)$, and let $(\sigma, \mathcal{W}), \mathcal{W} \subseteq \mathcal{A}\left(\mathrm{U}_{1}\right)$, be an irreducible representation of $\mathrm{U}_{1}\left(\mathbb{A}_{F, f}\right)$. Let $\chi_{\pi}$ and $\chi_{\sigma}$ be their respective central characters. We assume the following.

- $\chi_{\sigma}=\lambda \chi_{\pi}^{-1}$.

- If $v$ splits in $\mathcal{K}$ then $\sigma_{v} \simeq \tilde{\pi}_{v} \otimes \lambda_{v, 1}$ as representations of $\mathrm{GL}_{2}\left(F_{v}\right)$.

- We fix a finite set $S$ of primes outside of which $\lambda$ is unramified.

Let $\varphi \in \mathcal{V} \otimes \mathcal{W}$. We assume that

- if $v \notin S$ then $\varphi(h u, g)=\varphi(h, g)$ for $u \in H_{v}$;

- there is a character $\varepsilon$ of $T_{\mathrm{U}(1,1)}\left(\hat{\mathcal{O}}_{F}\right)$ and there is an ideal $N$ divisible only by primes in $S$ such that $\varphi(h, g k)=\varepsilon\left(\left({ }^{a_{k}} d_{k}\right)\right) \varphi(h, g)$ for all $k \in \mathrm{U}_{1}\left(\hat{\mathcal{O}}_{F}\right)$ satisfying $N \mid c_{k} .\left(k=\left(\begin{array}{ll}a_{k} & b_{k} \\ c_{k} & d_{k}\end{array}\right)\right)$.

Now for the group $\mathrm{U}_{1}$ we can similarly define Hecke operators $T_{v}^{U}$ for unramified split $v$ using the double coset action for $\left({ }^{\sigma_{v}}{ }_{1}\right)$ and $U_{v}$ operators for $v \mid p$ and the nearly ordinary projector $e_{U}$. The following lemma follows immediately from our assumptions for $\pi$ and $\sigma$. 
LEMma 90. Suppose that the above assumptions are valid. Then, for any $v \notin S$ that splits in $\mathcal{K}$,

$$
\left(\chi_{\pi, v}^{-1}\left(\left(\varpi_{v}, \varpi_{v}^{-1}\right)\right) \lambda_{v, 1}\left(\varpi_{v}\right) T_{v}^{H} \varphi\right)(h, u)=T_{v}^{U}(\varphi)(h, u),
$$

where $\lambda_{v}=\left(\lambda_{v, 1}, \lambda_{v, 2}\right)$ with respect to $\mathcal{K}_{v}=F_{v} \times F_{v}$ and $T_{v}^{H}$ and $T_{v}^{U}$ means Hecke operators on the groups $H$ and $\mathrm{U}_{1}$.

Now we consider the $p$-adic ordinary idempotents $e_{H}$ and $e$. For any $v \mid p$ suppose that $\varepsilon_{v}(k)=\varepsilon_{1, v}\left(a_{k}\right) \varepsilon_{2, v}\left(d_{k}\right)$. Suppose additionally that, for such $v$, the following hold.

- $\lambda_{v}$ is unramified at $v$.

- $\operatorname{cond}\left(\varepsilon_{2, v}\right)=\left(p^{r}\right), \operatorname{cond}\left(\varepsilon_{1, v}\right)=\left(p^{s}\right), r>s$ for any $v \mid p$.

- $p^{r} \| N$.

- $\varphi(h k, g)=\varepsilon_{2, v}^{-1}\left(a_{k}\right) \varepsilon_{1, v}^{-1}\left(d_{k}\right) \varphi(h, g)$ for $k=\left(k_{1}, k_{2}\right) \in H_{v}, p^{r} \mid c_{k_{1}}$.

LEMMA 91. The assumptions are as above. Then

$$
\left(e_{H} \varphi\right)(h,-)=e_{U}(\varphi)(h,-) .
$$

Proof. The proof is completely the same as that of [44, Lemma 13.2]. The point is that the vector with the prescribed level group action has to be the nearly ordinary one.

8.2.2. Consequences for Fourier coefficients. We return to the notation and setup of Section 6. In particular, $\mathcal{D}=(\varphi, \psi, \tau, \Sigma)$ is an Eisenstein datum. Let $\Theta_{i j k}(h, g):=\Theta_{\beta_{i j k}}\left(h, g ; \Phi_{\mathcal{D}, \beta_{i j k}, u_{i}}\right)$. Now we decompose each $\Theta_{i j k}(h, g)$ with respect to irreducible automorphic representations $\pi_{H}$ of $H_{i j k}\left(\mathbb{A}_{F, f}\right)$ :

$$
\Theta_{i j k}(h, g)=\sum_{\pi_{H}} \varphi_{\pi_{H}}^{(i j k)}(h, g) .
$$

Then, as in $[44,13.2 .2]$, using general consequences of theta correspondences in the split case we may decompose:

$$
\begin{aligned}
& \Theta_{i j k}(h, g)=\sum_{\left(\pi_{H}, \sigma\right)} \varphi_{\left(\pi_{H}, \sigma\right)}^{(i j k)}(h, g), \varphi_{\left(\pi_{H}, \sigma\right)}^{(i j k)} \in \pi_{H} \otimes \sigma, \\
& \sigma_{v} \simeq \tilde{\pi}_{H, v} \otimes \lambda_{v, 1} \text { as representations of } \mathrm{GL}_{2}\left(F_{v}\right) \text { for all } v \text { split in } \mathcal{K},
\end{aligned}
$$

and such $\varphi_{\left(\pi_{H}, \sigma\right)}^{(i j k)}(h, g)$ satisfies the assumptions about the nebentypus in the last subsubsection. 
For $i \in I_{1}$, let

$$
C_{\mathcal{D}, i j k}(h):=\bar{\tau}(\operatorname{det} h) C_{\mathcal{D}}\left(\beta_{i j k}, \operatorname{diag}\left(u_{i},{ }^{t} \bar{u}_{i}{ }^{-1}\right) ; h\right) \in \mathcal{A}_{H_{i j k}} .
$$

Recall that we have defined $A^{\prime}:=\sum_{i j k} A_{\beta_{i j k}}^{\prime}$.

Proposition 92. Let $\mathcal{L}=\left\{v_{1}, v_{2}, \ldots, v_{m}\right\}$ be a set of primes that split in $\mathcal{K}$ and do not belong to $\Sigma \cup \mathcal{Q}$. Let $P \in \mathbb{C}\left[X_{1}, \ldots, X_{m}\right]$. Let

$$
P_{H_{i j k}}:=P\left(\xi_{v_{1}, 1}\left(\varpi_{v_{1}}\right) T_{v_{1}}^{H_{i j k}}, \ldots, \xi_{v_{m}, 1}\left(\varpi_{v_{m}}\right) T_{v_{m}}^{H_{i j k}}\right)
$$

and $P_{\mathrm{U}_{1}}:=P\left(\xi_{v_{1}, 2} \lambda_{v_{1}, 1}^{-1}\left(\varpi_{v_{1}}\right) T_{v_{1}}^{U}, \ldots, \xi_{v_{m}, 2} \lambda_{v_{m}, 1}^{-1}\left(\varpi_{v_{m}}\right)\right)$. Then

$$
\begin{aligned}
& \frac{\sum_{i j k} e_{H_{i j k}} P_{H_{i j k}} C_{\mathcal{D}, i j k}(h) B_{\mathcal{D}}\left(\beta_{i j k}, h, u_{i}\right)^{-1}}{2^{-3 d}(2 i)^{d(k+1)} S(f)\left\langle f, \rho\left(\left(N^{-1}\right)\right) \tilde{f}^{c}\right\rangle} \\
& =\bar{\tau}(\operatorname{det} h)\left|\tilde{\delta}_{\mathcal{K}} \overline{\mathfrak{d}}_{1}\right|^{\kappa / 2-1} \xi\left(\tilde{\delta}_{\mathcal{K}} \overline{\mathfrak{d}}_{1}\right) \\
& \quad \times \frac{\left\langle B_{\mathcal{D}, 1} \mathcal{E}_{\mathcal{D}} \cdot B_{\mathcal{D}, 2}\left(\left(\left(^{\mathfrak{d}_{1}^{-1}} \overline{\mathfrak{d}}_{1}\right)\right) e_{U} P_{\mathrm{U}_{1}} A^{\prime}, \rho\left(\left(M_{\mathcal{D}}^{2} \tilde{D}_{\mathcal{K}^{\mathfrak{d}}}{ }^{-1}\right) \prod_{v \mid p}\left(p^{r_{v}}{ }^{-1}\right)\right) \tilde{f}^{c}\right\rangle\right.}{\left.\left\langle f, \rho\left(M^{-1}\right) \prod_{v \mid p}\left({p^{r v}}^{-1}\right)_{f}\right) \tilde{f}^{c}\right\rangle} .
\end{aligned}
$$

Proof. The proof follows from Lemmas 90 and 91 in the same way as for [44, Proposition 13.3] and $[44,13.2 .5]$. The assumptions for these lemmas follow from our explicit calculations in Section 5. Observe that $\rho\left(\left(\begin{array}{cc}\mathfrak{d}_{1}^{-1} & \\ & \overline{\mathfrak{d}}_{1}\end{array}\right)\right)$ commutes with $e P_{1}$.

Recall that we defined $A^{\prime}$ and $A$ in Equations (6.5) and (6.6). The following corollary follows easily from the above proposition by comparing the $\mathrm{GL}_{2}$ and $\mathrm{U}(1,1)$ Hecke eigenvalues for unramified split primes on automorphic forms with central character $\lambda \xi$, and applying Lemma 65.

Corollary 93. Let $P_{1}=P\left(T_{v_{1}}, \ldots, T_{v_{m}}\right)$ be a $\mathrm{GL}_{2}$ Hecke operator for the polynomial $P$ in the above proposition, and let e be the $\mathrm{GL}_{2}$ nearly ordinary projector. Then the last expression of the above proposition equals

$$
\begin{array}{r}
\bar{\tau}(\operatorname{det} h)\left|\tilde{\delta}_{\mathcal{K}} \overline{\mathfrak{d}}_{1}\right|^{\kappa / 2-1} \xi\left(\tilde{\delta}_{\mathcal{K}} \overline{\mathfrak{d}}_{1}\right) 2^{-u_{\mathcal{K}}}\left[\mathcal{O}_{\mathcal{K}}^{\times}: \mathcal{O}_{F}^{\times}\right]^{-1} B_{\mathcal{D}, 1} B_{\mathcal{D}, 2} \\
\quad \times\left\{\left\langle\mathcal{E}_{\mathcal{D}} \cdot \rho\left(\left(\begin{array}{ll}
\mathfrak{d}_{1}^{-1} & \\
& \overline{\mathfrak{d}}_{1}
\end{array}\right)\right) e P_{1} A, \rho\left(\left(M_{\mathcal{D}}^{2} \tilde{D}_{\mathcal{K}}{ }^{-1}\right)\right.\right.\right.
\end{array}
$$




$$
\begin{aligned}
& \left.\left.\times \prod_{v \mid p}\left(p^{r_{v}}-1\right) \tilde{f}^{c}\right\rangle_{\mathrm{GL}_{2}, \Gamma_{0}\left(M_{\mathcal{D}}^{2} \tilde{D}_{\mathcal{K}} \mathfrak{d} \prod_{v \mid p} p^{\left.r_{v}\right)}\right.}\right\} / \\
& \left\{\left\langle f,\left.\rho\left(\left(M^{-1}\right) \prod_{v \mid p}\left(p^{r^{-}}-1\right)_{f}\right) \tilde{f}^{c}\right|_{\mathrm{GL}_{2}, \Gamma_{0}\left(M \prod_{v \mid p} p^{r_{v}}\right)}\right\} .\right.
\end{aligned}
$$

Proof. It follows from the definition of $\alpha_{\lambda \xi}$ that for any $A^{\prime}$ on $\mathrm{U}(1,1)\left(\mathbb{A}_{F}\right)$ satisfying (*) and split $v$ outside $\Sigma$ we have

$$
(\lambda \xi)_{v, 2}\left(\varpi_{v}^{-1}\right) T_{v} \alpha_{\lambda \xi} A^{\prime}=\alpha\left(T_{v} A^{\prime}\right) .
$$

The corollary follows readily.

8.3. $\quad p$-adic properties of Fourier coefficients. In this subsection we put the operations above in $p$-adic families. Let $\mathbf{D}=(A, \mathbb{I}, \mathbf{f}, \psi, \xi, \Sigma)$ be a $p$-adic Eisenstein datum as in the last section, and $\mathbf{E}_{\mathbf{D}} \in \mathcal{M}_{\underline{a}, \text { ord }}\left(K_{\mathbf{D}}^{\prime}, \Lambda_{\mathbf{D}}\right)$ or $\tilde{\mathbf{E}}_{\mathbf{D}}$ be as there. For $x \in G\left(\mathbb{A}_{F, f}\right)$ with $x \in Q\left(\mathcal{O}_{F, v}\right)$ for all primes $v \mid p$ we let $\boldsymbol{c}_{\mathbf{D}}(\beta, x)$ $\in \Lambda_{\mathbf{D}}$ or $\tilde{\boldsymbol{c}}_{\mathbf{D}}(\beta, x) \in \Lambda_{\mathbf{D}}$ be the $\beta$-Fourier coefficient of $\mathbf{E}_{\mathbf{D}}$ or $\mathbf{b} \tilde{\mathbf{E}}_{\mathbf{D}}$ at $x$ (here $\mathbf{b}$ is defined in Remark 89). So, for $\phi \in \mathcal{X}_{\mathbf{D}}^{a}, c_{\mathcal{D}_{\phi}}(\beta, x):=\phi\left(\boldsymbol{c}_{\mathbf{D}}(\beta, x)\right)$ is the $\beta$-Fourier expansion at $x$ of a holomorphic Hermitian modular form $E_{\mathcal{D}_{\phi}}(Z, x)$. We define the $\tilde{c}_{\mathcal{D}_{\phi}}(\beta, x)$ correspondingly. Define

$$
\varphi_{\mathbf{D}, \beta, x, \phi}(h):=\chi_{\mathbf{f}} \psi_{\phi}^{-1} \xi_{\phi}(\operatorname{det} h) c_{\mathcal{D}_{\phi}}\left(\beta,\left(\begin{array}{cc}
h & \\
& t
\end{array}\right.\right.
$$

and

$$
\tilde{\varphi}_{\mathbf{D}, \beta, x, \phi}(h):=\chi_{\mathbf{f}} \psi_{\phi}^{-1} \xi_{\phi}(\operatorname{det} h) \tilde{c}_{\mathcal{D}_{\phi}}\left(\beta,\left(\begin{array}{cc}
h & \\
& { }^{t} \bar{h}^{-1}
\end{array}\right) x\right) .
$$

As in [44, 13.3.1], recall that $\beta_{i j k}=\left(\begin{array}{cc}b_{j} & \\ & q_{i} b_{k}\end{array}\right)$ and $u_{i}=\gamma_{0}\left(\begin{array}{cc}{ }^{1} & \\ a_{i}^{-1}\end{array}\right)$. For $h \in$ $\mathrm{GL}_{2}\left(\mathbb{A}_{\mathcal{K}, f}\right)$ with $h_{v} \in \mathrm{GL}_{2}\left(\mathcal{O}_{\mathcal{K}, v}\right)$ for all $v \mid p$, let

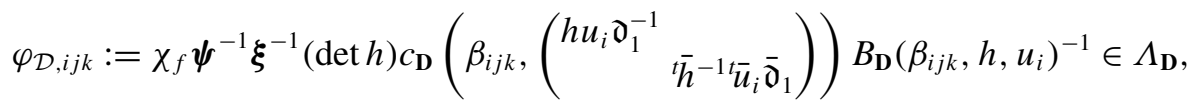

where $B_{\mathbf{D}}\left(\beta_{i j k}, h, u_{i}\right)$ is the element interpolating the $B_{\mathcal{D}_{\phi}}\left(\beta_{i j k}, h, u_{i}\right)$ defined in equation (6.9). Note that by our choices $B_{\mathbf{D}}\left(\beta_{i j k}, h, u_{i}\right)^{-1}$ is a unit in $\Lambda_{\mathbf{D}}$.

For $\phi \in \mathcal{X}_{\mathbf{D}}^{a}$ and $h \in \mathrm{GL}_{2}\left(\mathbb{A}_{\mathcal{K}, f}\right)$, let

$$
\varphi_{\mathbf{D}, i j k, \phi}(h):=\varphi_{\mathbf{D}, \beta_{i j k}, \operatorname{diag}\left(u_{i} \mathfrak{d}_{1}^{-1}, \bar{u}_{i}^{-1} \overline{\mathfrak{d}}_{1}\right), \phi}(h) .
$$


We define the $\tilde{\varphi}$ versions of the above objects correspondingly when the local Hecke algebra for $\mathbf{f}$ is not known to be Gorenstein. Now we have the following lemma interpolating the Hecke operators, completely as in [44, Lemma 13.4].

LEMMA 94. Let $\mathcal{L}:=\left\{v_{1}, \ldots, v_{m}\right\}$ be a finite set of primes that split in $\mathcal{K}$ and do not belong to $\Sigma \cup \mathcal{Q}$. Let $P \in \Lambda_{\mathbf{D}}\left[X_{1}, \ldots, X_{m}\right]$. For $h \in H_{i}\left(\mathbf{A}_{F, f}\right)$ with $h_{p} \in H_{i, p}$, there exists $\varphi_{\mathbf{D}, i}(\mathcal{L}, P ; h) \in \Lambda_{\mathcal{D}}$ such that the following hold.

(a) For all $\phi \in \mathcal{X}_{\mathcal{D}}^{a}$,

$$
\begin{aligned}
& \phi\left(\varphi_{\mathbf{D}, i j k}(\mathcal{L}, P ; h)\right) \\
& \quad=P_{\phi}\left(\xi_{\phi, v_{1}, 1}\left(\varpi_{v_{1}}\right) T_{v_{1}}^{H_{i j k}}, \ldots, \xi_{\phi, v_{m}, 1}\left(\varpi_{v_{m}}\right) T_{v_{m}}^{H_{i j k}}\right) e_{H_{i j k}} \varphi_{\mathbf{D}, i j k, \phi}(h),
\end{aligned}
$$

where $P_{\phi}$ is the polynomial obtained by applying $\phi$ to the coefficients of $P$.

(b) If $M \subseteq \Lambda_{\mathbf{D}}$ is a closed $\Lambda_{\mathbf{D}}$-submodule and $\varphi_{\mathbf{D}, i j k}(h) \in M$ for all $h$ with $h_{p} \in H_{i, p}$, then $\varphi_{\mathbf{D}, i j k}(\mathcal{L}, P ; h) \in M$.

Observe that the nebentypus of $\alpha_{\xi \lambda}(A)$ at $v \mid p$ are given by

$$
\varepsilon^{\prime}\left(\left(\begin{array}{ll}
a_{v} & \\
& d_{v}
\end{array}\right)\right) \rightarrow \mu_{1, v}\left(a_{v}\right) \mu_{2, v}\left(d_{v}\right) \tau_{1, v}^{-1} \tau_{2, v}^{-1}\left(d_{v}\right)
$$

for any $a_{v}, d_{v} \in \mathcal{O}_{F_{v}}^{\times}$. From the definition of the theta functions ( $q$-expansion) we know that $\alpha_{\xi \lambda}(A)$ is a $\Lambda_{\mathbf{D}}$-adic form. Also for each arithmetic weight $\phi$ the specialization at $\phi$ is a form of parallel weight two and the nebentypus at all $v \mid p$ only depend on the restriction of $\phi$ to

$$
R^{+}:=\mathbb{I}\left[\left[\Gamma_{\mathcal{K}}^{+}\right]\right] .
$$

Now let $\boldsymbol{g} \in M^{\text {ord }}\left(M_{\mathbf{D}}^{2} \tilde{D}_{\mathcal{K}}, 1 ; \Lambda_{W, A}\right)$ be a Hida family of forms which are new at primes not dividing $p$ and such that $g \otimes \chi_{\mathcal{K}}=\boldsymbol{g}$. Suppose also that the localization of the Hecke algebra at the maximal ideal corresponding the $\boldsymbol{g}$ is Gorenstein so that $\ell_{g}$ makes sense. Now following the remark of [44] before Proposition 13.5, one can change the weight homomorphism and view $g$ as an element of $M^{\text {ord }}\left(M_{\mathbf{D}}^{2} \tilde{D}_{\mathcal{K}}, 1 ; R^{+}\right)$(the space of $R^{+}$-adic families of nearly ordinary forms with tame level $M_{\theta}^{2} \tilde{D}_{\mathcal{K}}$ and trivial character) such that, at any $\phi, \boldsymbol{g}_{\phi}$ is a normalized nearly ordinary eigenform of parallel weight two and nebentypus at $v \mid p$ the same as $\alpha_{\xi \lambda}(A)$. Also as in [44] one can find a polynomial of the Hecke actions $P_{g}:=P\left(T_{v_{1}}, \ldots, T_{v_{m}}\right) \in \mathbb{T}^{\text {ord }}\left(M_{\mathbf{D}}^{2} \tilde{D}_{\mathcal{K}}, 1 ; R^{+}\right)$such that $P_{g}=a_{g} \ell_{g}$ with $0 \neq a_{g} \in R^{+}$.

With these preparations we can prove the following proposition in the same way as [44, Proposition 13.5]. 
PROPOSITION 95. Under the above hypotheses we have the following.

(1) If the local Hecke algebra for $\mathbf{f}$ is Gorenstein, then we have

$$
\sum_{i, j, k} \varphi_{\mathbf{D}, i j k}\left(\mathcal{L}, P_{g} ; 1\right)=\mathcal{A}_{\mathbf{D}, g} \mathcal{B}_{\mathbf{D}, g}
$$

with $\mathcal{A}_{\mathbf{D}, g} \in \mathbb{I}\left[\left[\Gamma_{\mathcal{K}}^{+}\right]\right]$and $\mathcal{B}_{\mathbf{D}, g} \in \mathbb{I}\left[\left[\Gamma_{\mathcal{K}}\right]\right]$ such that for all $\phi \in \mathcal{X}_{\mathbf{D}}^{\text {gen }}$ :

$$
\begin{aligned}
\phi\left(\mathcal{A}_{\mathbf{D}, g}\right)= & 2^{-u \mathcal{K}}\left[\mathcal{O}_{\mathcal{K}}^{\times}: \mathcal{O}_{F}^{\times}\right]^{-1}\left|\tilde{\delta}_{\mathcal{K}} \overline{\mathfrak{d}}_{1}\right|_{\mathcal{K}}^{/ / 2-1} \xi\left(\tilde{\delta}_{\mathcal{K}} \overline{\mathfrak{d}}_{1}\right) \phi\left(a_{g}\right) \eta_{\mathbf{f}_{\phi}} \\
& \frac{\left\langle B_{\mathbf{D}, 1} \mathcal{E}_{\mathcal{D}_{\phi}} \rho\left(\left({ }^{1} \mathfrak{d}\right)\right) \boldsymbol{g}_{\phi}, \rho\left(\left(M_{\mathcal{D}}^{2} \tilde{D}_{\mathcal{K}}{ }^{-1}\right) \prod_{v \mid p}\left({ }^{r_{v, \phi}}{ }^{-1}\right)\right) \boldsymbol{f}_{\phi}^{c}\right\rangle_{\mathrm{GL}_{2}}}{\left\langle f_{\phi}, \rho\left(\left(^{-1}\right) \prod_{v \mid p}\left(p^{r_{v, \phi}}-1\right)\right) f_{\phi}^{c}\right\rangle_{\mathrm{GL}_{2}}},
\end{aligned}
$$

and for $\phi \in \mathcal{X}^{a}$,

$$
\begin{aligned}
& \phi\left(\mathcal{B}_{\mathbf{D}, \boldsymbol{g}}\right)=\eta_{\boldsymbol{g}_{\phi}} \times \\
& \frac{\left\langle B_{\mathcal{D}, 2} \alpha_{\xi \lambda}\left(\sum_{i j k} \Theta_{\mathbf{D}_{\phi}, \beta_{i j k}} \otimes \xi_{\phi}\right), \rho\left(\left(M_{\mathcal{D}}^{2} \tilde{D}_{\mathcal{K}}{ }^{-1}\right) \prod_{v \mid p}\left(p^{r_{v, \phi}}{ }^{-1}\right)\right) \boldsymbol{g}_{\phi}^{c}\right\rangle_{\mathrm{GL}_{2}}}{\left\langle\boldsymbol{g}_{\phi}, \rho\left(\left(M_{\mathbf{D}}^{2} \tilde{D}_{\mathcal{K}}{ }^{-1}\right) \prod_{v \mid p}\left(p^{r_{v, \phi}}-1\right)\right) \boldsymbol{g}_{\phi}^{c}\right\rangle_{\mathrm{GL}_{2}}} .
\end{aligned}
$$

Furthermore, $\mathcal{A}_{\mathbf{D}, g} \neq 0$ (recall that $M_{\mathcal{D}_{\phi}}$ is independent of $\phi$ and thus we write $M_{\mathbf{D}}$ for it).

(2) In general, we have

$$
\sum_{i, j, k} \tilde{\varphi}_{\mathbf{D}, i j k}\left(\mathcal{L}, P_{g} ; 1\right)=\tilde{\mathcal{A}}_{\mathbf{D}, g} \mathcal{B}_{\mathbf{D}, g}
$$

where $\tilde{\mathcal{A}}_{\mathbf{D}, \mathbf{g}} \in F_{\mathbb{I}}\left[\left[\Gamma_{\mathcal{K}}^{+}\right]\right]$and $\phi\left(\tilde{\mathcal{A}}_{\mathbf{D}, \mathbf{g}}\right)$ is the expression in $(1)$ with $\eta_{\mathbf{f}_{\phi}}$ replaced by $\mathbf{b}_{\phi}$ and $\mathcal{B}_{\mathbf{D}, \mathbf{g}}$ the same as in (1).

Now we prove the following key proposition.

PROPOSITION 96. Let $A$ be the integer ring of a finite extension of $\mathbb{Q}_{p}, \mathbb{I}$ a local domain and a finite torsion-free $\Lambda_{W, A}$ or $\Lambda_{W, A}^{\text {para }}$-algebra, and $\mathbf{f} \in \mathcal{M}^{\text {ord }}(M, 1 ; \mathbb{I})$ an $\mathbb{I}$-adic newform such that (irred) and (dist) hold.

(i) Suppose that $\mathbb{T}_{\mathfrak{m}_{\mathfrak{f}}}$ is Gorenstein. Then, by possibly enlarging the $\Sigma$ in our Eisenstein datum, there exists an integer $M_{\mathbf{D}}$ as before and divisible by all 
primes dividing $\Sigma$ such that the following holds for the associated $\Lambda_{\mathbf{D}}$-adic Eisenstein series $\mathbf{E}_{\mathbf{D}}$ and the set

$$
\mathcal{C}_{\mathbf{D}}=\left\{c_{\mathbf{D}}\left(\beta_{i j k}, x\right) ; x \in G\left(\mathbb{A}_{F, f}\right) \cap Q\left(F_{p}\right)\right\}
$$

of Fourier coefficients of $\mathbf{E}_{\mathbf{D}}$. If $R \subseteq \Lambda_{\mathbf{D}}$ is any height-one prime containing $\mathcal{C}_{\mathbf{D}}$, then $R=P \Lambda_{\mathbf{D}}$ for some height-one prime $P \subset \mathbb{I}\left[\left[\Gamma_{\mathcal{K}}^{+}\right]\right]$.

(ii) In general (not assuming Gorensteinness), the conclusion in (i) is still true with the $\mathcal{C}_{\mathbf{D}}$ and $c_{\mathbf{D}}\left(\beta_{i j k}, x\right)$ replaced by $\tilde{\mathcal{C}}_{\mathbf{D}}$ and $\tilde{c}_{\mathbf{D}}\left(\beta_{i j k}, x\right)$.

Proof. We follow the proof of [44, Proposition 13.6] closely. As in [44], we only need to find an $M_{\mathbf{D}}$ so that there is a $\boldsymbol{g}$ with $\mathcal{B}_{\mathbf{D}, \boldsymbol{g}}$ a $p$-adic unit.

First we find an idele class character $\theta$ of $\mathbb{A}_{\mathcal{K}}^{\times}$such that

- $\theta_{\infty}(z)=\prod_{v \in \Sigma} z_{v}^{-1}$

- $\left.\theta\right|_{\mathbb{A}_{F}^{\times}}=|\cdot|_{F} \chi_{\mathcal{K} / F} ;$

- $\operatorname{Nm}\left(\mathfrak{f}_{\theta}\right)=\left(M_{\theta}^{2}\right)$ for some $M_{\theta} \in F^{\times}$prime to $p$ and such that $D_{\mathcal{K}} M \mid M_{\theta}$

- and $v \mid M_{\theta}$ for all $v \in \Sigma \backslash\{p\}$;

- for some $v \mid \tilde{D}_{\mathcal{K}}$, the anticyclotomic part of $\left.\theta\right|_{\mathcal{O}_{\mathcal{K}, q}} ^{\times}$has order divisible by $q_{v}$;

- $\Omega_{\infty}^{-2 \Sigma} L(1, \theta)$ is a $p$-adic unit, where $\Omega_{\infty}$ is the CM period defined in [16];

- $\theta_{v, 2}(p)-1$ is a $p$-adic unit for any $v \mid p$;

- $\theta_{\text {tor }}^{a}$ has order prime to $p$;

- the local character $\theta_{\text {tor }}^{a}$ is nontrivial over $\mathcal{K}_{\mathfrak{P}}^{\times}$for all $\mathfrak{P} \in \Sigma_{p}$; and

- the restriction of $\theta_{\text {tor }}^{a}$ to $\operatorname{Gal}\left(\bar{F} / \mathcal{K}\left[\sqrt{p^{*}}\right]\right)$ is nontrivial.

Here $\theta_{t o r}^{a}$ is the 'torsion part' (as defined in [18]) of the anticyclotomic part of $\theta^{a}:=\theta^{c} / \theta, p^{*}$ is $(-1)^{(p-1) / 2} p$.

The existence is proven in a similar way as in [44, Proposition 13.6], using the main theorem of [24] instead of results of Finis. (The result in [24, Theorem A] is not stated in the generality we need since he puts a condition $(\mathrm{C})$ there requiring that the nonsplit part of the conductor of the CM character is square free. But this condition is removed in a later paper of his paper; see [25, Section 6].) Write $g_{\theta}$ for the CM-eigenform corresponding to $\theta$. Now using the main result of [18] and [19] (we thank Hida for informing us his results in [19]), under the last three conditions 
above (which are put to apply Hida's result), we have that the Gorensteinness for $g_{\theta}$ is true and

$$
\eta_{g_{\theta}} \mid \frac{\left(g_{\theta}, g_{\theta}\right)}{\Omega_{\infty}^{2 \Sigma}}
$$

Thus

$$
\frac{L(1, \theta)^{2} / \Omega_{\infty}^{2 \Sigma}}{L(1, \theta)^{2} / \Omega_{\text {can }}}
$$

is $p$-adically integral where $\Omega_{\text {can }}$ is the canonical period associated to $g_{\theta}$.

The $g_{\theta}$ has parallel weight two, level $M_{\theta}^{2} \tilde{D}_{\mathcal{K}}$, and trivial neben character. Similar to [44, Proposition 13.6], we see that it satisfies (irred) and (dist). Let $g \in$ $\mathcal{M}^{\text {ord }}\left(M_{\theta}^{2} \tilde{D}_{\mathcal{K}}, \mathbf{1} ; R^{+}\right)$(see the discussion before Proposition 95) be the ordinary CM newform associated with $\theta$. (This is constructed in [22, page 133-134]. One needs to first construct the automorphic representation generated by some theta series and then pick up the nearly ordinary vector inside that representation space.) Recall that we have defined $A:=\alpha_{\xi \lambda}\left(\sum_{i, j, k} \Theta_{\beta_{i j k}} \otimes \xi\right)$ in Equation (6.6). Now we evaluate $\mathcal{B}_{\mathbf{D}, \mathbf{g}}$ at the $\phi$ which restricts trivially to the $W_{i, v}$ and $\Gamma_{\mathcal{K}}$. In this case, by checking the $q$-expansion (at the end of Section 6.10), the argument in $[44,11.9 .2]$ gives that up to a constant which is a $p$-adic unit (in fact the number of roots of unity in $\mathcal{O}_{\mathcal{K}}$ ) we have

$$
A_{\phi}=\left(B_{\mathcal{D}, 4}\right)_{\phi} E^{\prime}\left(\chi_{\mathcal{K}}\right) \rho\left(\left(M_{\mathcal{D}}^{2}-1\right)\right) E^{\prime}\left(\chi_{\mathcal{K}}\right),
$$

where $\left(B_{\mathcal{D}, 4}\right)_{\phi}=\left|M_{\mathcal{D}}^{2}\right|_{F}^{-1}\left|\delta_{\mathcal{K}}\right|_{\mathcal{K}} 2^{3 d} i^{-2 d}\left|\delta_{\mathcal{K}}\right|_{\mathcal{K}}^{1 / 2}$ which is a $p$-adic unit, and $A_{\phi}$ is the $A$ defined using the Eisenstein datum at $\phi$. Here

$$
E^{\prime}=\prod_{v \mid p}\left(1-p^{1 / 2}\left(\rho\left(\left(\begin{array}{ll}
1 & \\
& p
\end{array}\right)\right)\right)\right) E\left(\chi_{\mathcal{K}}\right)
$$

for $E\left(\chi_{\mathcal{K}}\right)$ being the weight-one Eisenstein series whose $L$-function is $L(F, s)$. $L\left(F, \chi_{\mathcal{K}}, s\right)$. We write

$$
h=E^{\prime}\left(\chi_{\mathcal{K}}\right) \rho\left(\left({M_{\mathcal{D}}^{2}}^{-1}\right)\right) E^{\prime}\left(\chi_{\mathcal{K}}\right) .
$$

Then the argument in [44, Proposition 13.6] tells us that

$$
\begin{aligned}
\left\langle h, \rho\left(\prod_{v \mid p}\left(\begin{array}{l}
-1 \\
\left.{ }^{-1}\right)_{v}\left(M_{\mathcal{D}} \tilde{D}_{\mathcal{K}}\right.
\end{array}{ }^{-1}\right)\right) g^{c}\right\rangle \\
=\frac{ \pm\left|\tilde{D}_{\mathcal{K}}\right|_{F} \prod_{v \mid p} \theta_{v, 2}(p)^{-2}}{i^{d}(-2 \pi i)^{2 d} \mathfrak{g}\left(\chi_{\mathcal{K}}\right)} L(1, \theta)^{2} \prod_{v \mid p}\left(1-\theta_{v, 2}(p)\right)^{3} .
\end{aligned}
$$


(In [44] they used [34] to construct the weight-one Eisenstein series with the required $q$-expansion when $F=\mathbb{Q}$, using the theory of analytic continuation of Eisenstein series. In the totally real field case we use, for example, [5, Section 6] for this generalization.)

Thus

$$
\phi\left(\mathcal{B}_{\mathbf{D}, \mathbf{g}}\right)=\frac{ \pm\left|\tilde{D}_{\mathcal{K}}\right|_{F} \prod_{v \mid p} \theta_{v, 2}(p)^{-2}}{i^{d}(-2 \pi i)^{2 d} \mathfrak{g}\left(\chi_{\mathcal{K}}\right) \Omega_{\mathrm{can}}} L(1, \theta)^{2} \prod_{v \mid p}\left(1-\theta_{v, 2}(p)\right)^{3} .
$$

By definition, $\phi\left(\mathcal{B}_{\mathbf{D}, \mathbf{g}}\right)$ is $p$-integral. But, as noted before, $\left(L(1, \theta)^{2}\right) / \Omega_{\text {can }}$ divides $\left(L(1, \theta)^{2}\right) / \Omega_{\infty}^{2 \Sigma_{\infty}}$, a $p$-adic unit. Thus itself must also be a $p$-adic unit. Therefore, $\mathcal{B}_{\mathbf{D}, \mathbf{g}}$ is a unit. This proves (i). Part (ii) follows similarly (note $\mathbf{b} \in F_{\mathbb{I}}^{\times}$for $\mathbf{b}$ defined in Remark 89).

\section{Proof of the main results}

\subsection{The Eisenstein ideal.}

9.1.1. Hecke operators. Let $K^{\prime}=K_{\Sigma}^{\prime} K^{\Sigma} \subset G\left(\mathbb{A}_{f}^{p}\right)$ be an open compact subgroup with $K^{\Sigma}=G\left(\hat{\mathcal{O}}_{F}^{\Sigma}\right)$ and such that $K:=K^{\prime} K_{p}^{0}$ is neat. The Hecke operators we are going to consider are at the unramified places and at primes dividing $p$. We closely follow $[44,9.5,9.6]$.

Unramified inert case. Let $v$ be a prime of $F$ inert in $\mathcal{K}$. Recall as in $[44,9.5 .2]$ that $Z_{v, 0}$ is the Hecke operator associated to the matrix $z_{0}:=\operatorname{diag}\left(\varpi_{v}, \varpi_{v}, \varpi_{v}\right.$, $\left.\varpi_{v}\right)$ by the double coset $K z_{0} K$ where $K$ is the maximal compact subgroup of $G\left(\mathcal{O}_{F, v}\right)$. Let $t_{0}:=\operatorname{diag}\left(\varpi_{v}, \varpi_{v}, 1,1\right), t_{1}:=\operatorname{diag}\left(1, \varpi_{v}, 1, \varpi_{v}^{-1}\right)$ and $t_{2}:=$ $\operatorname{diag}\left(\varpi_{v}, 1, \varpi_{v}^{-1}, 1\right)$. As in $[44,9.5 .2]$, we define

$$
\mathcal{R}_{v}:=\mathbb{Z}\left[X_{v}, q^{1 / 2}, q^{-1 / 2}\right]
$$

for $X_{v}$ being $T\left(F_{v}\right) / T\left(\mathcal{O}_{F_{v}}\right)$, and write [t] for the image of $t$ in $X_{v}$. Let $\mathcal{H}_{K}$ be the abstract Hecke ring with respect to the level group $K$. There is a map $\mathcal{S}_{K}: \mathcal{H}_{K} \rightarrow \mathcal{R}_{v}$ given by $\mathcal{S}_{K}(K g K)=\sum_{j \in J} \delta_{B}^{1 / 2}\left(t_{j}\right)\left[t_{j}\right]$ if $K g K=\bigsqcup_{j \in J} t_{j} n_{j} K$ for $t_{j} \in T\left(F_{v}\right), n_{j} \in N_{B}\left(F_{v}\right)$ and extend this map linearly. We define the Hecke operators $T_{i}$ for $i=1,2,3,4$ by requiring that

$$
1+\sum_{i=1}^{4} \mathcal{S}_{K}\left(T_{i}\right) X^{i}=\prod_{i=1}^{2}\left(1-q_{v}^{3 / 2}\left[t_{i}\right] X\right)\left(1-q_{v}^{3 / 2}\left[t_{i}\right]^{-1} X\right)
$$


is an equality of polynomials of the variable $X$. We also define

$$
Q_{v}(X):=1+\sum_{i=1}^{4} T_{i}\left(Z_{0} X\right)^{i} .
$$

Unramified split case. Suppose that $v$ is a prime of $F$ split in $\mathcal{K}$. In this case we define $z_{0}^{(1)}$ and $z_{0}^{(2)}$ to be $\left(\operatorname{diag}\left(\varpi_{v}, \varpi_{v}, \varpi_{v}, \varpi_{v}\right), 1\right)$ and $\left(1, \operatorname{diag}\left(\varpi_{v}, \varpi_{v}, \varpi_{v}\right.\right.$, $\left.\varpi_{v}\right)$ ) and define the Hecke operators $Z_{0}^{(1)}$ and $Z_{0}^{(2)}$ as above but replacing $z_{0}$ by $z_{0}^{(1)}$ and $z_{0}^{(2)}$. Let $t_{1}^{(1)}:=\operatorname{diag}\left(1,\left(\varpi_{v}, 1\right), 1,\left(1, \varpi_{v}^{-1}\right)\right), t_{2}^{(1)}:=\operatorname{diag}\left(\left(\varpi_{v}, 1\right), 1,(1\right.$, $\left.\left.\varpi_{v}^{-1}\right), 1\right)$. Define $t_{i}^{(2)}:=\bar{t}_{i}^{(1)}$ and $t_{i}=t_{i}^{(1)} t_{i}^{(2)}$ for $i=1,2$. We define $R_{v}$ and $\mathcal{S}_{K}$ in the same way as in the inert case. Then we define Hecke operators $T_{i}^{(j)}$ for $i=1,2,3,4$ and $j=1,2$ by requiring

$$
1+\sum_{i=1}^{4} \mathcal{S}_{K}\left(T_{i}^{(j)}\right) X^{i}=\prod_{i=1}^{2}\left(1-q_{v}^{3 / 2}\left[t_{i}^{(j)}\right] X\right)\left(1-q_{v}^{3 / 2}\left[t_{i}^{\left(j^{\prime}\right)}\right]^{-1} X\right)
$$

to be equalities of polynomials of the variable $X$. Here $j^{\prime}=3-j$ and the $\left[t_{i}^{(j)}\right]$ are defined similarly to the inert case. Now let $v=w \bar{w}$ for $w$ a place of $\mathcal{K}$. Define $i_{w}=1$ and $i_{\bar{w}}=2$. Then we define

$$
\begin{aligned}
& Q_{w}(X):=1+\sum_{i=1}^{4} T_{i}^{\left(i_{w}\right)}\left(Z_{0}^{\left(3-i_{w}\right)} X\right)^{i} . \\
& Q_{\bar{w}}(X):=1+\sum_{i=1}^{4} T_{i}^{\left(i_{w}\right)}\left(Z_{0}^{\left(3-i_{\bar{w}}\right)} X\right)^{i} .
\end{aligned}
$$

$p$-adic case. Let $t=\operatorname{diag}\left(p^{a_{1}}, p^{a_{2}}, p^{a_{4}}, p^{a_{3}}\right)$, and let $u_{t}$ be Hida's normalized operator defined in $[44,6.2 .2]$.

Let $\mathbf{h}_{\mathbf{D}}=\mathbf{h}_{\mathrm{D}}\left(K^{\prime}\right)$ be the reduced quotient of the universal ordinary cuspidal Hecke algebra which is defined by the ring of elements in $\operatorname{End}_{\Lambda_{\mathbf{D}}}\left(S^{\text {ord }}\left(K^{\prime}, \Lambda_{\mathbf{D}}\right)\right)$ generated by the Hecke operators $Z_{v, 0}, Z_{v, 0}^{(i)}, T_{i, v}, T_{i, v}^{(j)}, u_{t, v}$ defined above. This is a finite reduced $\Lambda_{\mathbf{D}}$-algebra. Now we define for each prime $w$ of $\mathcal{K}$ a polynomial $Q_{w, \mathbf{D}}(X)=\operatorname{det}\left(1-\rho_{\mathbf{D}}\left(\operatorname{Frob}_{w}\right) X\right)$ associated to Eisenstein series where $\rho_{\mathbf{D}}$ is the Galois representation defined in Section 9.2.2.

We define the Eisenstein ideal $I_{\mathbf{D}}$ (which is actually the kernel of homomorphism from the abstract Hecke algebra to $\Lambda_{\mathbf{D}}$ determined by the Eisenstein family) generated by the following.

- The coefficients of $Q_{w}(X)-Q_{w, \mathbf{D}}(X), Q_{\bar{w}}(X)-Q_{\bar{w}, \mathbf{D}}(X), Q_{v}(X)-Q_{v, \mathbf{D}}(X)$ for all finite places $v$ of $\mathcal{K}$ and not dividing a prime in $\Sigma$. 
- $Z_{v, 0}-\sigma_{\psi} \sigma_{\xi}^{-1}\left(\right.$ Frob $\left._{v}\right)$ for $v$ inert places outside $\Sigma$.

- $Z_{v, 0}^{(i)}-\boldsymbol{\sigma}_{\psi} \boldsymbol{\sigma}_{\xi}^{-1}\left(\right.$ Frob $\left._{w_{i}}\right)$ for all $v$ outside $\Sigma$ such that $v=w_{1} w_{2}$ is the factorization of $\mathcal{K}_{v}=F_{v} \times F_{v}$.

- For all $v \mid p, u_{t, v}-\lambda_{\mathbf{E}_{\mathbf{D}}}\left(u_{t, v}\right)$ with $t=\operatorname{diag}\left(p^{a_{1}}, p^{a_{2}}, p^{a_{4}}, p^{a_{3}}\right), a_{1} \leqslant \cdots \leqslant a_{4}$.

Here, $\sigma$ is the reciprocity map of class field theory normalized by the geometric Frobenius, and $\lambda_{\mathbf{E}_{\mathbf{D}}}$ is the Hecke eigenvalue for $u_{v, t}$ acting on $\mathbf{E}_{\mathbf{D}}$. These are elements in $\mathbb{I}\left[\left[\Gamma_{\mathcal{K}}\right]\right]$ (the $\lambda_{i, v}(p)$ can be expressed in terms of the Hecke eigenvalues of $U_{v}$ on $\mathbf{f}$ and the $\xi_{v, 1}(p)$ and $\xi_{v, 2}$ in our situation). We omit the precise formulas. We remark that the elements in $I_{\mathbf{D}}$ all annihilate $E_{\mathbf{D}}$. Note also that the factor $\Gamma_{\mathcal{K}}^{-}$in $\Lambda_{\mathbf{D}}$ corresponds to twisting everything by characters so our families are essentially parameterized by $\mathbb{I}\left[\left[\Gamma_{\mathcal{K}}\right]\right]$.

The structure map $\Lambda_{\mathbf{D}} \rightarrow \mathbf{h}_{\mathbf{D}} / I_{\mathbf{D}}$ is surjective, and we denote $\mathcal{E}_{\mathbf{D}} \subset \Lambda_{\mathbf{D}}$ to be kernel of this map so that

$$
\Lambda_{\mathbf{D}} / \mathcal{E}_{\mathbf{D}} \stackrel{\sim}{\rightarrow} \mathbf{h}_{\mathbf{D}} / I_{\mathbf{D}} .
$$

Recall that we have defined $\phi_{0}$ to be the point on the weight space in Section 7.3.1 such that the special $L$-value interpolated is $L\left(f_{2}, 1\right)$ where $f_{2}$ is an ordinary form in our Hida family of parallel weight two and trivial nebentypus at primes dividing $p$. (In fact this notion is a little bit ambiguous since we might have several $f_{2}$ inside the Hida family and what we are going to prove is true for any such point $\left.\phi_{0}.\right)$

Recall that we have chosen a $\boldsymbol{b} \in \mathbb{I}$ such that $\boldsymbol{b}\left(\phi_{0}\right) \neq 0$ and $\boldsymbol{b} \mathbf{1}_{\mathbf{f}}$ is an integral element of Hecke action. Then $\boldsymbol{b} \tilde{\mathcal{L}}_{\mathbf{D}}^{\Sigma}$ and $\boldsymbol{b} \tilde{\mathbf{E}}_{\mathbf{D}}$ are all integral. We have the following theorem which is the analog of [44, Theorem 6.17] in our situation.

THEOREM 97. If $P \subset \Lambda_{\mathbf{D}, \mathbb{I}}$ is a height-one prime of $\Lambda_{\mathbf{D}}$ contained in $\phi_{0}$ such that $\boldsymbol{b} \tilde{\boldsymbol{E}}_{\mathbf{D}}$ is nonzero modulo $P$ (that is, if the ideal generated by the Fourier coefficient of $\boldsymbol{b} \tilde{\mathbf{E}}_{\mathbf{D}}$ is not contained in $P$ ), and if $P$ is not a pullback of a height-one prime from $\mathbb{I}\left[\left[\Gamma_{\mathcal{K}}^{+}\right]\right]$, then

$$
\operatorname{ord}_{P}\left(\mathcal{E}_{\mathbf{D}}\right) \geqslant \operatorname{ord}_{P}\left(\tilde{\mathcal{L}}_{\mathbf{D}}^{\Sigma}\right) .
$$

If we do not assume $P$ to be contained in $\phi_{0}$ but assume $\mathbb{T}_{\mathfrak{m}_{f}}$ is Gorenstein, then

$$
\operatorname{ord}_{P}\left(\mathcal{E}_{\mathbf{D}}\right) \geqslant \operatorname{ord}_{P}\left(\mathcal{L}_{\mathbf{D}}^{\Sigma}\right) .
$$

Proof. The proof is completely the same as that of [44, Theorem 6.17]. By Theorem 27 we can find an $\mathbf{F} \in M_{\text {ord }}^{0}\left(K, \Lambda_{\mathbf{D}}\right)$ such that $\mathbf{F} \equiv \mathbf{b} \tilde{\mathbf{E}}_{\mathbf{D}}\left(\bmod \mathcal{L}_{\mathbf{D}}\right)$ in terms of $q$-expansion. Then for some $\beta \in S_{2}(F)$ and $x \in \mathrm{U}(2,2)\left(\mathbb{A}_{F}\right)$ unramified at primes dividing $p$ we have that the Fourier coefficient $c(\beta, x ; \mathbf{F})$ is not in $P$. 
Suppose that $P^{r} \| \tilde{\mathcal{L}}_{\mathbf{D}}^{\Sigma}$. Then the map $\mu: \mathbf{h}_{\mathbf{D}} \mapsto \Lambda_{\mathbf{D}}$ defined by $\mu(h)=c(\beta$, $x ; h \mathbf{F}) / c(\beta, x ; \mathbf{F})$ is $\Lambda_{\mathbf{D}}$-linear and, for any $h \in \mathbf{h}_{\mathbf{D}}$, we have

$$
c(\beta, x ; h \mathbf{F}) \equiv c\left(\beta, x ; h \mathbf{E}_{\mathbf{D}}\right) \equiv \lambda_{\mathbf{D}}(h) c\left(\beta, x ; \mathbf{E}_{\mathbf{D}}\right) \equiv \lambda_{\mathbf{D}}(h) c(\beta, x ; \mathbf{F})\left(\bmod P^{r}\right) .
$$

Here $\lambda_{\mathbf{D}}(h)$ is the Hecke eigenvalue of $h$ for $\mathbf{E}_{\mathbf{D}}$, and we note that $\boldsymbol{b} \notin P$. Thus $\mu$ gives

$$
\mathbf{h}_{\mathbf{D}} / I_{\mathbf{D}} \rightarrow \Lambda_{\mathbf{D}, P} / \mathcal{E}_{\mathbf{D}} \Lambda_{\mathbf{D}, P} \rightarrow \Lambda_{\mathbf{D}, P} / P^{r} \Lambda_{\mathbf{D}, P}
$$

This gives the first claim of the theorem. The second is proved similarly.

\subsection{Galois representations.}

9.2.1. Galois theoretic argument. In this subsection, for ease of reference we axiomatize the results from [44, Section 4], which are used to construct elements in the Selmer group.

Let $G$ be a group and $C$ a ring, and let $r: \rightarrow \operatorname{Aut}_{C}(V)$ be a representation of $G$ with $V \simeq C^{n}$. This can be extended to $r: C[G] \rightarrow \operatorname{End}_{C}(V)$. For any $x \in C[G]$, define

$$
\operatorname{Ch}(r, x, T):=\operatorname{det}(\mathrm{id}-r(x) T) \in C[T] .
$$

Let $\left(V_{1}, \sigma_{1}\right)$ and $\left(V_{2}, \sigma_{2}\right)$ be two $C$ representations of $G$. Assume that both are defined over a local henselian subring $B \subseteq C$. We say that $\sigma_{1}$ and $\sigma_{2}$ are residually disjoint modulo the maximal ideal $\mathfrak{m}_{B}$ if there exists $x \in B[G]$ such that $\operatorname{Ch}\left(\sigma_{1}\right.$, $x, T) \bmod \mathfrak{m}_{B}$ and $\operatorname{Ch}\left(\sigma_{2}, x, T\right) \bmod \mathfrak{m}_{B}$ are relatively prime in $\kappa_{B}[T]$, where $\kappa_{B}:=B / \mathfrak{m}_{B}$.

Let $H$ be a group with a decomposition $H=G \rtimes\{1, c\}$ with $c \in H$ an element of order two normalizing $G$. For any $C$ representations $(V, r)$ of $G$ we write $r^{c}$ for the representation defined by $r^{c}(g)=r(\operatorname{cgc} c)$ for all $g \in G$.

Polarizations: Let $\theta: G \rightarrow \mathrm{GL}_{L}(V)$ be a representation of $G$ on a vector space $V$ over a field $L$, and let $\psi: H \rightarrow L^{\times}$be a character. We assume that $\theta$ satisfies the $\psi$-polarization condition:

$$
\theta^{c} \simeq \psi \otimes \theta^{\vee}
$$

By a $\psi$-polarization of $\theta$ we mean an $L$-bilinear pairing $\Phi_{\theta}: V \times V \rightarrow L$ such that

$$
\Phi_{\theta}\left(\theta(g) v, v^{\prime}\right)=\psi(g) \Phi_{\theta}\left(v, \theta^{c}(g)^{-1} v^{\prime}\right) .
$$

Let $\Phi_{\theta}^{t}\left(v, v^{\prime}\right):=\Phi_{\theta}\left(v^{\prime}, v\right)$, which is another $\psi$-polarization. We say that $\psi$ is compatible with the polarization $\Phi_{\theta}$ if

$$
\Phi_{\theta}^{t}=-\psi(c) \Phi_{\theta}
$$


Suppose that the following hold.

(1) $A_{0}$ is a profinite $\mathbb{Z}_{p}$ algebra and a Krull domain.

(2) $P \subset A_{0}$ is a height-one prime and $A=\hat{A}_{0, P}$ is the completion of the localization of $A_{0}$ at $P$. This is a discrete valuation ring.

(3) $R_{0}$ is local reduced finite $A_{0}$-algebra.

(4) $Q \subset R_{0}$ is a prime such that $Q \cap A_{0}=P$ and $R=\hat{R}_{0, Q}$.

(5) There exist ideals $J_{0} \subset A_{0}$ and $I_{0} \subset R_{0}$ such that $I_{0} \cap A_{0}=J_{0}, A_{0} / J_{0}=$ $R_{0} / I_{0}, J=J_{0} A, I=I_{0} R, J_{0}=J \cap A_{0}$ and $I_{0}=I \cap R_{0}$.

(6) $G$ and $H$ are profinite groups; we have subgroups $D_{i} \subset G$ for $i=1, \ldots, d$.

The set-up. Suppose the we have the following data:

(1) a continuous character $v: H \rightarrow A_{0}^{\times}$;

(2) a continuous character $\xi: G \rightarrow A_{0}^{\times}$such that $\bar{\chi} \neq \bar{v} \bar{\chi}^{-c}$, and let $\chi^{\prime}:=v \chi^{-c}$;

(3) a representation $\rho: G \rightarrow \operatorname{Aut}_{A}(V), V \simeq A^{n}$, which is a base change from a representation over $A_{0}$, such that

(a) $\rho^{c} \simeq \rho^{\vee} \otimes v$,

$\bar{\rho}$ is absolutely irreducible,

$\rho$ is residually disjoint from $\chi$ and $\chi^{\prime}$;

(4) a representation $\sigma: G \rightarrow \operatorname{Aut}_{R \otimes_{A} F}(M), M \simeq\left(R \otimes_{A} F\right)^{m}$ with $m=n+2$, which is defined over the image of $R_{0}$ in $R$, such that

(a) $\sigma^{c} \simeq \sigma^{\vee} \otimes \nu$,

(b) $\operatorname{tr} \sigma(g) \in R$ for all $g \in G$,

(c) for any $v \in M, \sigma(R[G]) v$ is a finitely generated $R$-module;

(5) a proper ideal $I \subset R$ such that $J:=A \cap I \neq 0$, the natural map $A / J \rightarrow R / I$ is an isomorphism, and

$$
\operatorname{tr} \sigma(g) \equiv \chi^{\prime}(g)+\operatorname{tr} \rho(g)+\chi(g) \bmod I
$$

for all $g \in G$;

(6) $\rho$ is irreducible and $v$ is compatible with $\rho$; 
(7) (local conditions for $\rho$ ) for each $v \mid p$ there is a $G_{v}$-stable; sub- $A_{0}$-module; $A_{0^{-}}$ modules $V_{0, v}^{+} \subset V_{0}$ such that $V_{0, v}^{+}$and $V_{0, v}^{-}:=V_{0, v} / V_{0, v}^{+}$are free $A_{0}$ modules;

(8) (local conditions for $\sigma$ ) for each $v \mid p$ there is a $G_{v}$-stable; sub- $R \otimes_{A} F$-module $M_{v}^{+} \subseteq M$ such that $M_{v}^{+}$and $M_{v}^{-}:=M / M_{v}^{+}$are free $R \otimes_{A} F$ modules;

(9) (compatibility with the congruence condition) Assume that for all $x \in R\left[G_{v}\right]$, we have congruence relation:

$$
\operatorname{Ch}\left(M_{v}^{+}, x, T\right) \equiv \operatorname{Ch}\left(V_{v}^{+}, x, T\right)(1-T \chi(x)) \bmod I
$$

(then we automatically have

$$
\left.\operatorname{Ch}\left(M_{v}^{-}, x, T\right) \equiv \operatorname{Ch}\left(V_{v}^{-}, x, T\right)\left(1-T \chi^{\prime}(x)\right) \bmod I\right) ;
$$

(10) for each $F$-algebra homomorphism $\lambda: R \otimes_{A} F \rightarrow K, K$ a finite field extension of $F$, the representation $\sigma_{\lambda}: G \rightarrow \mathrm{GL}_{m}\left(M \otimes_{R \otimes F} K\right)$ obtained from $\sigma$ via $\lambda$ is either absolutely irreducible or contains an absolutely irreducible two-dimensional sub $K$-representation $\sigma_{\lambda}^{\prime}$ such that $\operatorname{tr} \sigma_{\lambda}^{\prime}(g) \equiv$ $\chi(g)+\chi^{\prime}(g) \bmod I$.

One defines the Selmer groups $\mathbf{X}_{H}\left(\chi^{\prime} / \chi\right):=\operatorname{ker}\left\{H^{1}\left(G, A_{0}^{*}\left(\chi^{\prime} / \chi\right)\right) \rightarrow H^{1}(D\right.$, $\left.\left.A_{0}^{*}\left(\chi^{\prime} \chi\right)\right)\right\}^{*}$ and $\mathbf{X}_{G}\left(\rho_{0} \otimes \chi^{-1}\right):=\operatorname{ker}\left\{H^{1}\left(G, V_{0} \otimes_{A_{0}} A_{0}^{*}\left(\chi^{-1}\right)\right) \rightarrow H^{1}\left(D, V_{0}^{-} \otimes_{A_{0}}\right.\right.$ $\left.\left.A_{0}^{*}\left(\chi^{-1}\right)\right)\right\}^{*}$. Let $\mathrm{Ch}_{H}\left(\chi^{\prime} \chi\right)$ and $\mathrm{Ch}_{G}\left(\rho_{0} \otimes \chi^{-1}\right)$ be their characteristic ideals as $A_{0}$ modules.

PROPOSITION 98. Under the above assumptions, if $\operatorname{ord}_{P}\left(\mathrm{Ch}_{H}\left(\chi^{\prime} / \chi\right)\right)=0$ then

$$
\operatorname{ord}_{P}\left(\mathrm{Ch}_{G}\left(\rho_{0} \otimes \chi^{-1}\right)\right) \geqslant \operatorname{ord}_{P}(J) \text {. }
$$

This can be proved as in the proof of [44, Corollary 4.16].

We record here an easy lemma about Fitting ideals and characteristic ideals which will be useful in proving the main conjectures.

LemMa 99. Let $A$ be a Krull domain and $T$ a finitely generated A-module. Suppose that $f \in A$ is such that, for any height-one primes $P$ of $A$, if $\operatorname{ord}_{P}\left(\operatorname{Fitt}_{A} T\right) \geqslant \operatorname{ord}_{P}(f)$, then $\operatorname{char}_{A}(T) \subset(f)$.

Proof. For any $g \in \operatorname{char}_{A}(T)$, the assumption and the definition for characteristic ideals ensure that, for any height-one prime $P, \operatorname{ord}_{P}(g / f) \geqslant 0$. Since $A$ is normal this implies that $g / f \in A$. Thus $g \in(f)$. 
9.2.2. Galois representations. Now we are going to apply the results in the last subsubsection to our situation. First we define a semisimple representation

$$
\rho_{\mathbf{D}}:=\boldsymbol{\sigma}_{\psi}^{c} \epsilon^{-3} \oplus\left(\rho_{\mathbf{f}} \otimes \boldsymbol{\sigma}_{\xi^{-c}} \boldsymbol{\sigma}_{\psi^{c}} \epsilon^{-2}\right) \oplus \epsilon^{-1} \operatorname{det} \rho_{\mathbf{f}} \boldsymbol{\sigma}_{\xi^{\prime}}^{-1} \boldsymbol{\sigma}_{\psi}^{c} .
$$

Recall that here $\sigma$ means the reciprocity map. This is the Galois representation associated to the Eisenstein family by Proposition 33.

On the other hand, recall that we have fixed some prime to $p$ level $K^{p}$, and let $K=\prod_{v \mid p} K_{v}^{0} K^{p}$. By an argument completely the same as that in [44, Proposition 7.3], using results of [41], [36], and [42], there exists a pseudorepresentation $T_{K^{p}}^{\Sigma}$ : $G_{\mathcal{K}} \rightarrow \mathbf{h}_{\mathbf{D}}$ such that for each irreducible cuspidal automorphic representation $\pi_{\phi}$ on $\mathrm{GU}(2,2)_{/ F}$ of weight $\underline{k}_{\phi}$ which is a classical specialization at some $\phi \in \operatorname{Spech}_{\mathbf{D}}$ then

$$
\operatorname{tr}\left(R_{p}(\pi)\right)=\phi \circ T_{K^{p}}^{\Sigma} .
$$

As in [44, 7.3] we let $\mathbf{T}_{\mathbf{D}}$ be the pseudocharacter $T_{K^{p}}^{\Sigma}$. We have defined $\mathbf{h}_{\mathbf{D}}$, and we let $B_{\mathbf{D}}:=\mathbf{h}_{\mathbf{D}} \otimes_{\Lambda_{\mathbf{D}}} F_{\Lambda_{\mathbf{D}}}$. Let $\mathbf{h}_{\mathbf{D}, \mathfrak{m}}$ be the localization of $\mathbf{h}_{\mathbf{D}}$ at the maximal ideal containing the Eisenstein ideal.

For any prime $v \mid p$ of $F$ we let $T_{\mathbf{f}, v}^{+} \subseteq T_{\mathbf{f}}$ be the rank-one I-summand of $T_{\mathbf{f}}$ that is $G_{v}$-stable. Given a height-one prime $P$ of $\Lambda_{\mathbf{D}}$ containing $\mathcal{E}_{\mathbf{D}}$, with the notations in the last subsubsection we let

- $H:=G_{F, \Sigma}, G:=G_{\mathcal{K}, \Sigma}, c=$ the usual complex conjugation;

- $A_{0}:=\Lambda_{\mathbf{D}}, A:=\hat{\Lambda}_{\mathbf{D}, P}$;

- $J_{0}:=\mathcal{E}_{\mathbf{D}}, J:=\mathcal{E}_{\mathbf{D}} A$;

- $R_{0}:=\mathbf{h}_{\mathbf{D}, \mathfrak{m}}, I_{0}:=I_{\mathbf{D}}$

- $Q \subset R_{0}$ is the inverse image of $P \bmod \mathcal{E}_{\mathcal{D}}$ under $\mathbf{h}_{\mathbf{D}, \mathfrak{m}} \rightarrow \mathbf{h}_{\mathbf{D}, \mathfrak{m}} / I_{\mathbf{D}}=\Lambda_{\mathbf{D}} / \mathcal{E}_{\mathbf{D}}$;

- $R:=\hat{\mathbf{h}}_{\mathbf{D}, Q}, I:=I_{\mathbf{D}} R$;

- $V_{0}:=T_{\mathbf{f}} \otimes_{\mathbb{I}} \Lambda_{\mathbf{D}}, \rho:=\rho_{\mathbf{f}} \otimes \boldsymbol{\sigma}_{\xi}^{-c} \boldsymbol{\sigma}_{\psi}^{c} \epsilon^{-2}$;

- for any $v \mid p, V_{0, v}^{+}:=T_{\mathbf{f}, v}^{+} \otimes_{\mathbb{I}} A_{0}, V_{0, v}^{-}:=\left(T_{\mathbf{f}} / T_{\mathbf{f}, v}^{+}\right) \otimes_{A_{0}} A$;

- $V=V_{0} \otimes_{A_{0}} A, \rho=\rho_{0} \otimes_{A_{0}} A, V_{v}^{ \pm}:=V_{0, v}^{ \pm} \otimes_{A_{0}} A$;

- $\chi^{\prime}:=\epsilon^{-1} \operatorname{det} \rho_{\mathrm{f}} \boldsymbol{\sigma}_{\xi^{\prime}}^{-1} \boldsymbol{\sigma}_{\psi}^{c}, v:=\sigma_{\psi^{\prime}} \boldsymbol{\sigma}_{\xi^{\prime}}^{-1} \epsilon^{-4}$;

- $\chi:=\sigma_{\psi}^{c} \epsilon^{-3}$ so $\chi^{\prime}=v \chi^{-c}$;

- $M:=\left(R \otimes_{A} F_{A}\right)^{4} ; F_{A}$ is the field of fractions of $A$; and

- we let $\sigma$ be the representation on $M$ obtained from $\mathbf{T}_{\mathbf{D}}$ in the same way as $[44,7.3]$. 
Recall that $\mathcal{T}:=\left(T_{\mathbf{f}} \otimes_{\mathbb{I}} \mathbb{I}\left[\left[\Gamma_{\mathcal{K}}\right]\right]\right)\left(\boldsymbol{\varepsilon}_{\mathcal{K}}^{-c}\right) \otimes \epsilon$ and $\mathcal{T}_{v}^{+}:=\left(T_{\mathbf{f}, v}^{+} \otimes_{\mathbb{I}} \mathbb{I}\left[\left[\Gamma_{\mathcal{K}}\right]\right]\right)\left(\boldsymbol{\varepsilon}_{\mathcal{K}}^{-c}\right) \otimes \epsilon$ for each $v \mid p$. Let $\operatorname{char}_{\mathcal{K}}^{\Sigma}(\mathbf{f}) \subset \mathbb{I}\left[\left[\Gamma_{\mathcal{K}}\right]\right]$ be the characteristic ideal of the dual Selmer $\operatorname{group} X_{\mathcal{K}}^{\Sigma}\left(\mathcal{T},\left.\mathcal{T}_{v}^{+}\right|_{v \mid p}\right)$.

THEOREM 100. Suppose that $\mathbb{I}$ is an integrally closed domain. Let $P_{0} \subset \mathbb{I}\left[\left[\Gamma_{\mathcal{K}}\right]\right]$ be a height-one prime that is not a pullback of one of $\mathbb{I}\left[\left[\Gamma_{\mathcal{K}}^{+}\right]\right]$, and let $P=P_{0} \Lambda_{\mathbf{D}}$ be the height-one prime of $\Lambda_{\mathbf{D}}$ it generates. Suppose also that

$V^{+} \oplus A(\chi)$ and $V^{-} \oplus A\left(\chi^{\prime}\right)$ modulo $P$ do not have common irreducible pieces.

Then

$$
\operatorname{ord}_{P_{0}}\left(\operatorname{char}_{\mathcal{K}}^{\Sigma}(\mathbf{f})\right) \geqslant \operatorname{ord}_{P}\left(\mathcal{E}_{\mathbf{D}}\right)
$$

Proof. One just applies Proposition 98. The condition (10) there is guaranteed by an argument similar to that in [44, Theorem 7.6]: we use the modularity-lifting results in [45] for ordinary Galois representations satisfying (irred) and (dist) and Harris' result that there are no (CAP) forms when the weight $\underline{k}$ is sufficiently regular. We also use the main conjecture for totally real field $F$ proven in [52] to conclude that $\operatorname{ord}_{P}\left(\mathrm{Ch}_{H}\left(\chi^{\prime} / \chi\right)\right)=0$. (Since the $p$-adic $L$-function for a Hecke character involves only the cyclotomic direction and is nonzero, it is not in $P$. By [52] we know that the characteristic ideal is bounded by this $p$-adic $L$-function and thus is not contained in $P$.)

\subsection{Proof of the main results.}

THEOREM 101. Suppose that $p \geqslant 5$. Let L be a finite extension of $\mathbb{Q}_{p}$ and $\mathbb{I} a$ local normal domain and a finite integral extension of $\Lambda_{W}^{\mathrm{para}}$. Let $\mathbf{f}$ be an $\mathbb{I}$-adic nearly ordinary eigenform of tame level $M$ (a finite idele) with trivial character, such that some specialization of it is an ordinary cusp form of even parallel weight $\kappa_{0} \geqslant 2$. Suppose that $M=M^{+} M^{-}$with $M^{+}$divisible only by primes split in $\mathcal{K}$ and $M^{-}$divisible only by primes inert in $\mathcal{K}$, and that $\mathcal{K}$ satisfies assumption $(\triangle)$ defined in the introduction. Suppose also that the following hold.

- $\left(\operatorname{irred}_{\mathcal{K}}\right)$ and (dist) hold for $\mathbf{f}$.

- The assumptions of Theorem 8 hold.

- $M^{-}$is square free and its number of prime factors is congruent to d modulo 2.

- The reduction $\bar{\rho}_{\mathbf{f}}$ of $\rho_{\mathbf{f}}$ modulo the maximal ideal of $\mathbb{I}$ is ramified at all $v \mid M^{-}$. 
Let $\Sigma$ be any finite set of primes. Then

$$
\operatorname{char}_{\mathcal{K}_{\infty}}^{\Sigma}(\mathbf{f}) \subseteq\left(\mathcal{L}_{\mathbf{f}, \mathcal{K}}^{\Sigma}\right)
$$

Proof. We first consider a $\Sigma$ as before (containing all the bad primes) so that the $\Sigma$-imprimitive $p$-adic $L$-function is integral. By Theorems 97 and 100 we have the inequalities for the orders of any height-one prime which is not the pullback of a height-one prime of $\mathbb{I}\left[\left[\Gamma_{\mathcal{K}}^{+}\right]\right]$. Now suppose that $P$ is a height-one prime which is the pullback of a height-one prime of $\mathbb{I}\left[\left[\Gamma_{\mathcal{K}}^{+}\right]\right]$. If the module of dual Selmer group is not torsion then the conclusion is trivially true. Suppose that it is torsion. We use the idea of the argument in [13]. We first look at a long exact sequence coming from the Poitou-Tate long exact sequence

$$
\begin{aligned}
0 \rightarrow H_{\Sigma^{*}}^{1}\left(\mathcal{K}, T_{\mathbf{f}}^{\vee} \otimes \Lambda_{\mathcal{K}}\left(\boldsymbol{\varepsilon}_{\mathcal{K}}^{c} \cdot \epsilon^{-1}\right)\right) & \rightarrow H_{f}^{1}\left(\mathcal{K}, T_{\mathbf{f}}^{\vee} \otimes \Lambda_{\mathcal{K}}\left(\boldsymbol{\varepsilon}_{\mathcal{K}}^{c} \cdot \epsilon^{-1}\right)\right) \\
& \rightarrow \prod_{v \in \Sigma \backslash\{v \mid p\}} H_{f}^{1}\left(\mathcal{K}_{v}, T_{\mathbf{f}}^{\vee} \otimes \Lambda_{\mathcal{K}}\left(\boldsymbol{\varepsilon}_{\mathcal{K}}^{c} \cdot \epsilon^{-1}\right)\right) \\
& \rightarrow X_{\mathbf{f}, \mathcal{K}}^{\Sigma} \rightarrow X_{\mathbf{f}, \mathcal{K}} \rightarrow 0
\end{aligned}
$$

Here we use the standard notation $H_{f}^{1}$ for the local Selmer conditions (finite part). We write the subscript $\Sigma^{*}$ to mean take the $H_{f}^{1}$ at primes outside $\Sigma \backslash\{v \mid p\}$ but take $\{0\} \subseteq H_{f}^{1}(\mathcal{K},-)$ at $\Sigma \backslash\{v \mid p\}$. On the one hand, it is easy to see that $H_{f}^{1}\left(\mathcal{K}, T_{\mathbf{f}} \otimes \Lambda_{\mathcal{K}}\right)$ is a torsion-free $\mathbb{I}\left[\left[\Gamma_{\mathcal{K}}\right]\right]$ module from assumption (irred $\left.\mathcal{K}_{\mathcal{K}}\right)$. On the other hand, it is torsion by the assumption that $X_{\mathbf{f}, \mathcal{K}}^{\Sigma}$ is torsion and an easy control argument. So it has to be zero. By [12, Proposition 2.4] we see that the characteristic ideal of $H_{f}^{1}\left(\mathcal{K}_{v}, T_{\mathbf{f}} \otimes \Lambda_{\mathcal{K}}\right)$ is exactly the local Euler factor of our $p$-adic $L$-function at $v$ (note that $\mathcal{K}_{\infty}$ contains the cyclotomic extension so the conclusion in [12] is true in our situation as well). The inequality for the order of $P$ follows from Lemma 87. This finishes the proof for such choices of $\Sigma$. The result for general $\Sigma$ follows from this and a similar argument using the PoitouTate exact sequences.

Now we prove the following.

THEOREM 102. Let $p$ be a rational odd prime that splits completely in F. Let $f$ be a Hilbert modular form over $F$ of even parallel weight $\kappa_{0}$ and trivial character. Suppose that

(i) $f$ is ordinary at all primes of $F$ dividing $p$;

(ii) assumption (irred) holds for $f$; 
(iii) if $[F: \mathbb{Q}]$ is even and the global sign of $f$ if -1 , then the automorphic representation of $f$ is special in at least one finite place.

Suppose that Conjecture 6 is true for $F, p, \kappa=\kappa_{0}$ and any $g$ satisfying the assumption of Conjecture 6. If the central critical value $L\left(f, \kappa_{0} / 2\right)=0$, then the Selmer group $H_{f}^{1}\left(F, \rho_{f}^{*}\left(\left(2-\kappa_{0}\right) / 2\right)\right.$ ) is infinite. (So if $f$ has weight two then the theorem is unconditional.)

Note that assumption (dist) is automatically true in this case.

Proof. We only need to prove the theorem in the case when the root number for $f$ is +1 , since otherwise it is a well-known result of Nekovar [37, Theorem 12.2.3] (which crucially uses the work of S. Zhang). Condition (iii) is put by Nekovar's work.

First suppose that $d=[F: \mathbb{Q}]$ is even. Then we choose an imaginary quadratic extension $\mathcal{K}$ of $F$ so that $\mathcal{K} / F$ is split at all primes at which $f$ is ramified and such that $L\left(f, \chi_{\mathcal{K} / F}, \kappa_{0} / 2\right) \neq 0$ where $\chi_{\mathcal{K} / F}$ is the quadratic character of $\mathbb{A}_{F}^{\times}$associated to $\mathcal{K} / F$. This is possible by a well-known result of Waldspurger [48], [49]. Then the $S(1)$ defined in [4, page 123] consists of exactly all the infinite places, and since $d$ is even we are in the definite case there.

The (normalized ordinary) form $f$ belongs to a Hida family. (This is wellknown to experts, see [14].) Recall we have defined the point $\phi_{0}$ in $\operatorname{Spec} \Lambda_{\mathbf{D}}$ (Section 7.3.1). We remark that later we will use $\phi_{0}$ to denote the point (or prime) not only of $\operatorname{Spec} \Lambda_{\mathbf{D}}$ but also subspaces of it.

We do not know the Gorenstein properties for the local Hecke algebra associated with $\mathbf{f}$ in general, so we have to use $1_{\mathbf{f}}$ instead of $\ell_{\mathbf{f}}$ everywhere (recall they are defined in Section 7.2) and the nonintegral $p$-adic $L$-functions $\tilde{\mathcal{L}}_{\mathbf{f}, \mathcal{K}, 1}^{\Sigma}$ (in $F_{\mathbb{I}} \otimes_{\mathbb{I}} \mathbb{I}\left[\left[\Gamma_{\mathcal{K}}\right]\right]$ actually) and nonintegral Klingen Eisenstein series $\tilde{\mathbf{E}}_{\mathbf{D}}$. Suppose that $\tilde{\mathcal{L}}_{\mathbf{f}, \mathcal{K}, 1}^{\Sigma}=h / g$. It follows from Remark 89 that we may choose $g \in \mathbb{I}$ so that $g\left(\phi_{0}\right) \neq 0$. Start with the one-dimensional family of cyclotomic twists of $f$, that is, the subspace $\operatorname{Spec}_{L}\left[\left[\Gamma_{\mathcal{K}}^{+}\right]\right]$defined by the natural map $\Lambda_{\mathbf{D}, \mathbb{I}} \rightarrow \mathcal{O}_{L}\left[\left[\Gamma_{\mathcal{K}}^{+}\right]\right]$ where the map $\mathbb{I} \rightarrow \mathcal{O}_{L}$ is the specialization map at $f$. Since $L\left(f, \kappa_{0} / 2\right)=0$, $h\left(\phi_{0}\right)=0$ by our Remark 83. Then there is a height-one prime $P_{0}$ of $A\left[\left[\Gamma_{\mathcal{K}}^{+}\right]\right]$ contained in $\phi_{0}$ and containing the image of $h$ in $\mathcal{O}_{L}\left[\left[\Gamma_{\mathcal{K}}^{+}\right]\right]$.

Now we consider the specialization from $\mathbb{I}\left[\left[\Gamma_{\mathcal{K}}\right]\right]$ to $\mathcal{O}_{L}\left[\left[\Gamma_{\mathcal{K}}^{+}\right]\right]$. Note that any minimal prime of $\mathbb{I}\left[\left[\Gamma_{\mathcal{K}}\right]\right]$ containing $h$ will be height one. So we can find $P_{1}$ a height-one prime of $\mathcal{O}_{L}\left[\left[\Gamma_{\mathcal{K}}^{+}\right]\right]$contained in $\phi_{0}$ and containing the image of $h$ in $\mathcal{O}_{L}\left[\left[\Gamma_{\mathcal{K}}^{+}\right]\right]$such that $\operatorname{Supp} P_{0} \subset \operatorname{Supp} P_{1}$. Note also that $P_{1}$ does not contain $g$ since $g\left(\phi_{0}\right) \neq 0$. In Theorem 86 we have seen that (NV1) is satisfied in our situation and thus $h$ is not contained in any height-one prime of $\mathbb{I}\left[\left[\Gamma_{\mathcal{K}}^{+}\right]\right]$contained in $\phi_{0}$. So $P_{1}$ is not the pullback of a height-one prime of $\mathbb{I}\left[\left[\Gamma_{\mathcal{K}}^{+}\right]\right]$. Then Proposition 96(i) 
and Theorem 97 gives

$$
1 \leqslant \operatorname{ord}_{P} \tilde{\mathcal{L}}_{\mathbf{D}}^{\Sigma} \leqslant \operatorname{ord}_{P}\left(\mathcal{E}_{\mathbf{D}, \mathbb{I}}\right)
$$

By Theorem 100,

$$
\operatorname{ord}_{P_{1}} \operatorname{Fitt}_{\mathbf{f}, \mathcal{K}, 1}^{\Sigma} \geqslant 1 \text {. }
$$

Then we need to specialize back to $\mathcal{O}_{L}\left[\left[\Gamma_{\mathcal{K}}^{+}\right]\right]$. Using the control theorem for Selmer groups (results in Section 2.5.1, Proposition 15, Corollary 16, 17), we have $\operatorname{ord}_{P_{0}} \operatorname{Fitt}_{f, \mathcal{K}_{\infty}^{+}, 1}^{\Sigma} \geqslant 1$. By results of [12], we know that at primes $v \in \Sigma \backslash\{p\}$ the module of the finite part of the dual local Galois cohomology group has characteristic ideal generated by the local Euler factor of the corresponding $p$ adic $L$-functions, which is not contained in $\phi_{0}$. So we get $\operatorname{ord}_{P_{0}}$ Fitt $_{f, \mathcal{K}_{\infty}^{+}, 1} \geqslant 1$. We apply the control results in [11] and get that $H_{f}^{1}\left(\mathcal{K}, \rho_{f}^{*}\left(\left(2-\kappa_{0}\right) / 2\right)\right)$ is infinity. However by Lemma 14 this Selmer group is the product of Selmer groups for $f$ and $f \otimes \chi_{\mathcal{K}}$. By Conjecture 6 and our choice of $\mathcal{K}$, we know that $H_{f}^{1}(F$, $\left.\rho_{f \otimes \chi_{\mathcal{K}}}^{*}\left(\left(2-\kappa_{0}\right) / 2\right)\right)$ is finite. So our theorem is true.

Finally we assume $d$ is odd. Then again by Waldspurger's result we can find a real quadratic character $\chi_{F^{\prime} / F}$ such that $F^{\prime}$ is split at all primes at which $f$ is ramified and $L\left(f, \chi_{F^{\prime} / F}, \kappa_{0} / 2\right) \neq 0$. We consider $f_{F^{\prime}}$ the base change of $f$ to $F^{\prime}$. Then $\left[F^{\prime}: \mathbb{Q}\right]$ is even, and we deduce that at least one of $H_{f}^{1}\left(F, \rho_{f}^{*}\left(\left(2-\kappa_{0}\right) / 2\right)\right)$ and $H_{f}^{1}\left(F, \rho_{f}^{*} \otimes \chi_{F^{\prime} / F}\left(\left(2-\kappa_{0}\right) / 2\right)\right)$ is infinite. But by Conjecture 6 we know that $H_{f}^{1}\left(F, \rho_{f}^{*} \otimes \chi_{F^{\prime} / F}\left(\left(2-\kappa_{0}\right) / 2\right)\right)$ is finite. So $H_{f}^{1}\left(F, \rho_{f}^{*}\left(\left(2-\kappa_{0}\right) / 2\right)\right)$ must be infinite.

Finally we prove that the main theorem of [44] is true without one of the technical local assumptions (namely the existence of an $\ell \| M$ with $\left.\bar{\rho}\right|_{G_{\mathbb{Q}_{\ell}}}$ ramified), using the base-change trick as in the above theorem.

THEOREM 103. Suppose that $p \geqslant 5$. Let $f \in S_{\kappa}(M, L), \kappa \geqslant 2,2 \mid \kappa, p \nmid M$ and $L \subset \overline{\mathbb{Q}}_{p}$ a finite extension of $\mathbb{Q}_{p}$, be a p-ordinary cuspidal eigenform with trivial central character. Suppose that

- (irred) and (dist) hold for $\rho_{f}$;

then, for any set of primes $\Sigma$,

$$
\operatorname{char}_{\mathbb{Q}_{\infty}, L}^{\Sigma}(f)=\left(\mathcal{L}_{f}^{\Sigma}\right)
$$

in $\Lambda_{\mathbb{Q}, \mathcal{O}_{L}} \otimes_{\mathbb{Z}_{p}} \mathbb{Q}_{p}$. If, furthermore,

- there is an $\mathcal{O}_{L}$-basis of $T_{f}$ with respect to which the image of $\rho_{f}$ contains $\mathrm{SL}_{2}\left(\mathbb{Z}_{p}\right)$, and 
- there exists a real quadratic extension $F / \mathbb{Q}$ such that

- $p$ is unramified in $F$,

- any prime $\ell$ dividing $M$ such that $\ell \equiv-1(\bmod p)$ is inert in $F$, and any other prime divisors of $M$ is split in $F$, and

- the canonical period of $f$ over $F$ is a p-adic unit times the square of its canonical period over $\mathbb{Q}$,

then the equality holds in $\Lambda_{\mathbb{Q}, \mathcal{O}_{L}}$.

Proof. We choose a quadratic real extension $F$ of $\mathbb{Q}$ with $p$ unramified and consider the base change $f_{F}$ of $f$ to $F$. Assume that any prime $\ell$ dividing $M$ such that $\ell \equiv-1(\bmod p)$ is inert in $F$, and that any other prime divisor of $M$ is split in $F$. We need to know the Gorensteinness for the base change to $F$. Since $p \geqslant 5$, this follows from [6]. In order to apply Theorem 86 we have to check the assumptions in Theorem 8 . The first and second assumptions are trivially true. The third condition in Fujiwara's theorem is true since the base change to $F$ of a minimal modular lifting of $\bar{\rho}_{f}$ gives a minimal modular lifting for $\bar{\rho}_{f_{F}}$ (by our choice of $F$ ). The fourth assumption is empty since $d=2$. The last one is also guaranteed by our choice of $F$. We choose a quadratic imaginary field $\mathcal{K}$ over $F$ such that all primes dividing the conductors of $f$ or $F$ are split. So (NV2) is satisfied for any $v \mid p$. Thus we have that the one divisibility for the three-variable main conjecture is true. The theorem can be proved in the same way as in [44], using the control theorem for Selmer groups, results in [12] and results of Kato (note that the one-variable (cyclotomic) Selmer group for $f$ over $\mathcal{K}$ splits into the products of four Selmer groups for modular forms over $\mathbb{Q}$ and thus Kato's results apply). Note also that the assumption of image containing $\mathrm{SL}_{2}\left(\mathbb{Z}_{p}\right)$ is unchanged by taking quadratic twists. The assumption on the canonical periods for base change is used to ensure that the $p$-adic $L$-function of the base change splits into $p$-adic $L$-functions over $\mathbb{Q}$.

\section{Acknowledgements}

This paper is part of the author's Princeton University thesis under the supervision of Professor Christopher Skinner. We thank him for suggesting the problem, patiently answering many questions about his joint paper [44] with Eric Urban, and giving many helpful suggestions. We also thank Eric Urban, Shouwu Zhang, Haruzo Hida, and Ming-Lun Hsieh for useful communications, and the anonymous referees for carefully reading this paper and pointing out some errors and flaws in it. 


\section{References}

[1] S. Bloch and K. Kato, ' $L$-functions and Tamagawa numbers of motives', in The Grothendieck Festschrift, Vol. I, Progress in Mathematics, 86 (Birkhauser, Boston, MA, 1990), 333-400.

[2] M. Chida and M.-L. Hsieh, 'Special values of anticyclotomic $L$-functions for modular forms', Crelle's J. (to appear), preprint as of 06/16/13, arXiv:1204.2427.

[3] M. Chida and M.-L. Hsieh, 'On the anticyclotomic Iwasawa main conjecture for modular forms', Compos. Math. 151(5) (2015), 863-897.

[4] C. Cornut and V. Vatsal, 'Nontriviality of Rankin-Selberg $L$-functions and CM points', in L-Functions and Galois Representations, Durham, July 2004, LMS Lecture Note Series, 320 (Cambridge University Press, Cambridge, 2007), 121-186.

[5] P. Deligne and K. Ribet, 'Values of abelian $L$-functions at negative integers over totally real fields', Invent. Math. 59 (1980), 227-286.

[6] F. Diamond, 'On the Hecke action on the cohomology of Hilbert-Blumenthal surfaces', Contemp. Math. 210 (1998), 71-84.

[7] E. Eischen, M. Harris, J. Li and C. Skinner, ' $p$-adic $L$-functions for Unitary Shimura Varieties (II)', in preparation.

[8] G. Faltings and C.-L. Chai, Degeneration of Abelian Varieties, Ergebnisse der Math., 22 (Springer, New York, 1990).

[9] K. Fujiwara, 'Deformation rings and Hecke algebras in the totally real case', Preprint, 2006, arXiv:math/0602606.

[10] R. Greenberg, 'Iwasawa theory and p-adic deformations of motives', in Proc. on Motives held at Seattle, 1994.

[11] R. Greenberg, 'Iwasawa theory for elliptic curves', in Arithmetic Theory of Elliptic Curves (Springer, Berlin, Heidelberg, 1999), 51-144.

[12] R. Greenberg and V. Vinayak, 'On the Iwasawa invariants of elliptic curves', Invent. Math. 142(1) (2000), 17-63.

[13] R. Greenberg, 'Surjectivity of the global-to-local map defining a Selmer Group', Kyoto J. Math. 50(4) (2010), 853-888.

[14] H. Hida, 'On $p$-adic Hecke algebras for $\mathrm{GL}_{2}$ over totally real fields', Ann. of Math. (2) 128 (1988), 295-384.

[15] H. Hida, 'On $p$-adic $L$-functions of GL(2) $\times$ GL(2) over totally real fields', Ann. Inst. Fourier 40 (1991), 311-391.

[16] H. Hida, p-adic Automorphic Forms on Shimura Varieties, Springer Monographs in Mathematics (Springer, New York, 2004).

[17] H. Hida, 'Non-vanishing modulo $p$ of Hecke L-values', in Geometric Aspects of Dwork Theory (Walter de Gruyter, Berlin, 2004), 735-784.

[18] H. Hida, 'Anticyclotomic main conjectures', Doc. Math. Extra Volume Coates (2006), 465-532.

[19] H. Hida, 'Quadratic exercises in Iwasawa theory', Int. Math. Res. Not. IMRN 2009 (2009), 912-952.

[20] H. Hida, 'Irreducibility of the Igusa tower over unitary Shimura varieties', in On Certain LFunctions, Clay Mathematics Proceedings, 13 (American Mathematical Society, Providence, 2011), 187-203.

[21] H. Hida and J. Tilouine, 'Anti-cyclotomic Katz $p$-adic $L$-functions and congruence modules', Ann. Sci. Éc. Norm. Super. (4) 26(2) (1993), 189-259.

[22] H. Hida and J. Tilouine, 'On the anticyclotomic main conjecture for CM fields', Invent. math. 117 (1994). 
[23] M.-L. Hsieh, 'Eisenstein congruence on unitary groups and Iwasawa main conjecture for CM fields', J. Amer. Math. Soc. 27(3) (2014), 753-862.

[24] M.-L. Hsieh, 'On the non-vanishing of Hecke L-values modulo p', Amer. J. Math. 134(6) (2012), 1503-1539.

[25] M.-L. Hsieh, 'On the -invariant of anticyclotomic $p$-adic $L$-functions for CM fields', J. reine angew. Math. 688 (2014), 67-100.

[26] P.-C. Hung, 'On the non-vanishing $\bmod \ell$ of central $L$-values with anti-cyclotomic twists of modular forms', Preprint, 2014, available at https://sites.google.com/site/pinchihung0926/ho me/papers.

[27] K. Kato, ' $p$-adic Hodge theory and values of zeta functions of modular forms', Cohomologies p-adiques et applications arithmetiques'. III. Astérisque No. 295 (2004), ix, 117-290.

[28] S. S. Kudla, 'Splitting metaplectic covers of dual reductive pairs', Israel J. Math. 87(1-3) (1994), 361-401.

[29] K.-W. Lan, Arithmetic Compactifications of PEL-type Shimura Varieties, London Mathematical Society Monographs, 36 (Princeton University Press, Princeton, 2013).

[30] E. Lapid and S. Rallis, 'On the local factors of representations of classical groups in automorphic representations', in L-Functions and Applications: Progress and Prospects, Ohio State Univ. Math. Res. Inst. Publ., 11 (de Gruyter, Berlin, 2005), 309-359.

[31] M. Longo, 'On the Birch and Swinnerton-Dyer conjecture for modular elliptic curves over totally real fields', Ann. Fourier 56(3) (2006), Chartres: L'Institut, 1950.

[32] M. Longo, 'Anticyclotomic Iwasawa's main conjecture for Hilbert modular forms', Comment. Math. Helv. 87(2) (2012), 303-353.

[33] B. Mazur and A. Wiles, 'Class fields of abelian extensions of Q', Invent. Math. 76 (1984), 179-330.

[34] T. Miyake, Modular Forms (Springer, Berlin, 1989).

[35] C. Moglin and J.-L. Waldspurger, Spectral Decomposition and Eisenstein Series. Une paraphrase de l'ecriture, Cambridge Tracts in Mathematics, 113 (Cambridge University Press, Cambridge, 1995).

[36] S. Morel, On the Cohomology of Certain Non-compact Shimura Varieties, Annals of Mathematics Studies, 173 (Princeton University Press, Princeton, 2010).

[37] J. Nékovar, Selmer Complexes, Astérisque, 310 (Société mathématique de France, Paris, 2006).

[38] J. Nékovar, 'Level raising and anticyclotomic Selmer groups for Hilbert modular forms of weight two', Canad. J. Math. 64(3) (2012), 588-668.

[39] G. Shimura, Euler Products and Eisenstein Series, CBMS Regional Conference Series in Mathematics, 93 (American Mathematical Society, Providence, RI, 1997).

[40] G. Shimura, Arithmeticity in the Theory of Automorphic Forms, Mathematical Surveys and Monographs, 82 (American Mathematical Society, Providence, RI, 2000), x+302 pp.

[41] S.-W. Shin, 'Galois representations arising from some compact Shimura varieties', Ann. of Math. (2) 173(3) (2011), 1645-1741.

[42] C. Skinner, 'Galois representations associated with unitary groups over $\mathbb{Q}$ ', Algebra Number Theory 6(8) (2012), 1697-1717.

[43] C. Skinner and E. Urban, 'Vanishing of $L$-functions and Ranks of Selmer groups', in Proceedings of the International Congress of Mathematics, Vol. II (European Mathematical Society, Zurich, 2006), 473-500.

[44] C. Skinner and E. Urban, 'The Iwasawa main conjectures for GL2', Invent. Math. 195(1) (2014), 1-277. 
[45] C. Skinner and A. Wiles, 'Nearly ordinary deformations of residually irreducible representations', Ann. Fac. Sci. Toulouse, Math. (6) 10 (2001), 185-215.

[46] R. Taylor, 'On Galois representations associated to Hilbert modular forms', Invent. Math. 98.2 (1989), 265-280.

[47] J. Tilouine and E. Urban, 'Several-variable $p$-adic families of Siegel-Hilbert cusp eigensystems and their Galois representations', Ann. Sci. Éc. Norm. Supér. (4) 32 (1999), 499-574, no longer published by Elsevier.

[48] J.-L. Waldspurger, 'Sur les coefficients de Fourier des formes modulaires de poids demientier', J. Math. Pures Appl. 60 (1981), 375-484.

[49] J.-L. Waldspurger, 'Correspondances de Shimura et quaternions', Forum Math. 3 (1991), 219-307.

[50] X. Wan, 'Families of nearly ordinary Eisenstein series on unitary groups', submitted, 2013.

[51] A. Wiles, 'On ordinary $\lambda$-adic representations associated to modular forms', Invent. Math. 94(3) (1988), 529-573.

[52] A. Wiles, 'The Iwasawa conjecture for totally real fields', Ann. of Math. (2) 131 (1990).

[53] A. Wiles, 'Elliptic curves and Fermat's Last Theorem', Ann. of Math. (2) 141 (1995), 443-551.

[54] X. Yuan, S.-W. Zhang and W. Zhang, The Gross-Zagier formula on Shimura Curves, Annals of Mathematics Studies, 184 (Princeton University Press, Princeton, 2013).

[55] S. Zhang, 'Heights of Heegner points on Shimura curves', Ann. of Math. (2) 153 (2001), 27-147.

[56] S. Zhang, 'Gross-Zagier formula for GL(2). II. Heegner points and Rankin L-series', Math. Sci. Res. Inst. Publ., 49 (Cambridge University Press, Cambridge, 2004), 191-214. 